\title{
Lignite Derived Humic Substances for Treatment of Acid Mine Drainage
}

A thesis submitted in partial fulfilment

\author{
of the requirements for the \\ Master of Engineering \\ at the
}

University of Canterbury

William Olds

December 2011 



\section{Table of Contents}

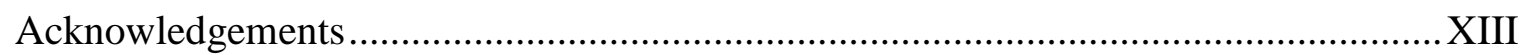

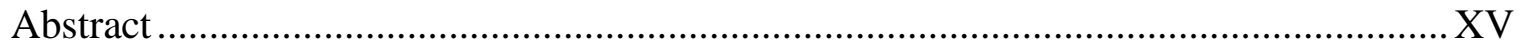

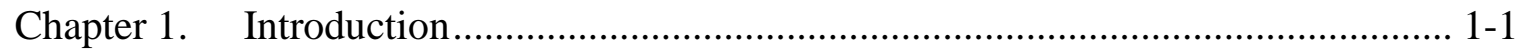

1.1 Statement of the Problem .............................................................................. 1-2

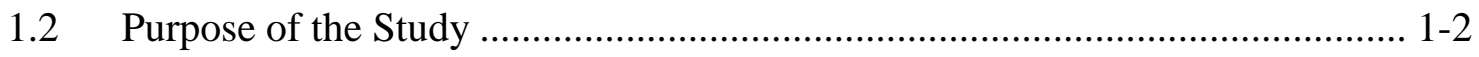

1.3 Significance to the Field ........................................................................ 1-4

Chapter 2. Literature Review........................................................................ 2-1

2.1 Background - AMD Evolution and Toxicity ........................................ 2-2

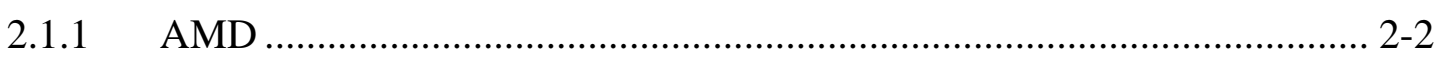

2.1.1.1 Pyrite Oxidation ..................................................................... 2-2

2.1.1.2 AMD Variability ................................................................. 2-4

2.1.1.3 Stockton AMD ............................................................................. 2-5

2.1.2 Environmental Impact/toxicity .................................................... 2-6

2.1.2.1 Acidity and Metal Toxicity ........................................................ 2-6

2.1.2.2 Downstream Impact................................................................ 2-8

2.2 Standard Methods of AMD Treatment............................................................ 2-9

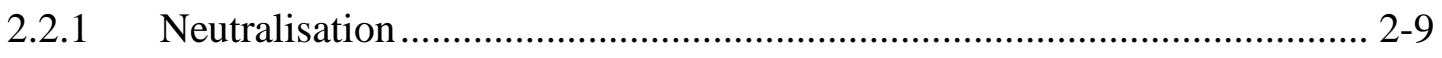

2.2.1.1 Hydroxide Precipitation.......................................................... 2-9

2.2.1.2 Inorganic Complexation ..................................................... 2-10

2.2.1.3 Trace Metal Adsorption/co-precipitation .................................... 2-12

2.2.1.4 Alkalinity Reagents ....................................................... 2-13

2.2.2 Treatment Processes.................................................................. 2-15

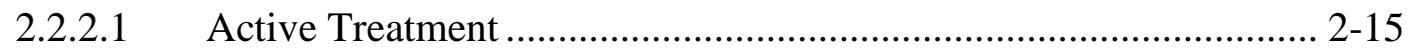

2.2.2.2 Passive Treatment ....................................................................... 2-19 
2.2.3 ANZECC Guidelines ........................................................................ 2-21

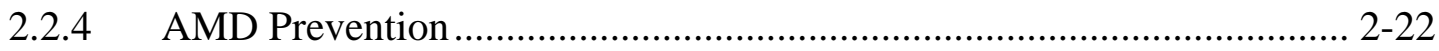

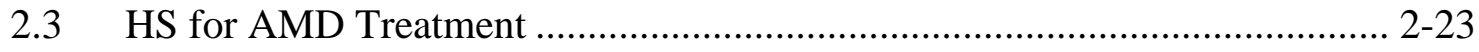

2.3.1 Humic Substance Properties ............................................................. 2-23

2.3.2 HS-Metal Complexation ......................................................... 2-28

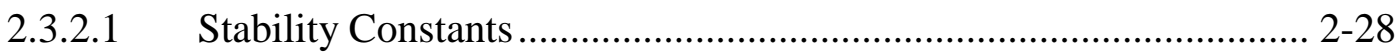

2.3.2.2 HS Complexation Mechanisms ................................................. 2-30

2.3.3 HS Application to Dissolved Metal Removal...................................... 2-31

2.3.3.1 Lignite Derived HS Complexation Capacity …............................. 2-31

2.3.4 Application of HS ................................................................... 2-32

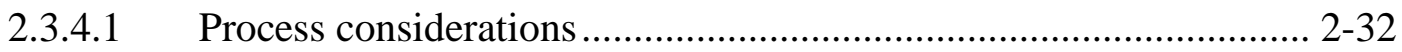

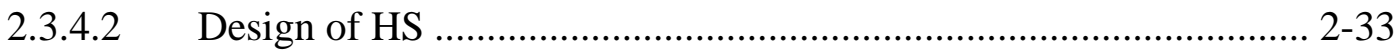

2.4 Lignite-derived HS for AMD Remediation ............................................ 2-34

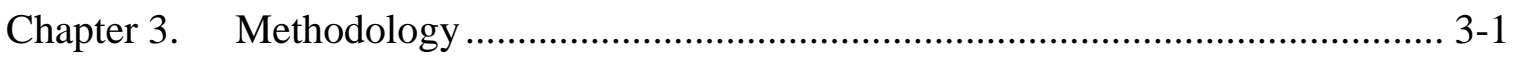

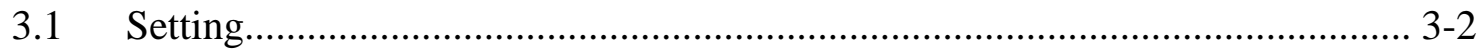

3.1.1 Sample Collection....................................................................... 3-3

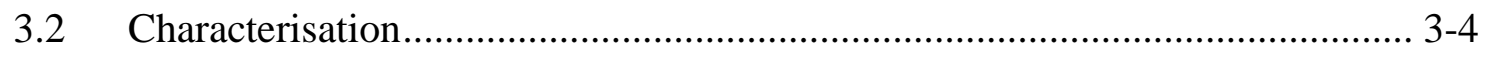

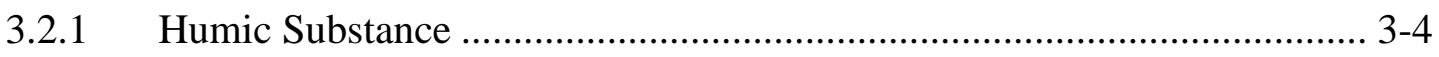

3.2.1.1 CHON .............................................................................. $3-4$

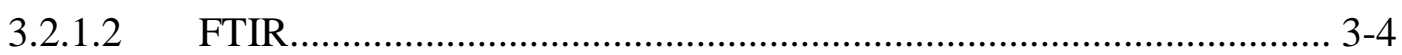

3.2.1.3 NMR Spectroscopy …................................................................. 3-5

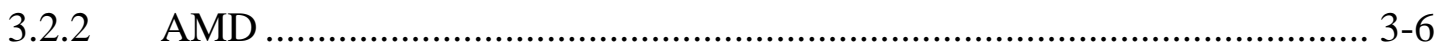

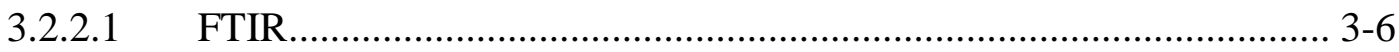

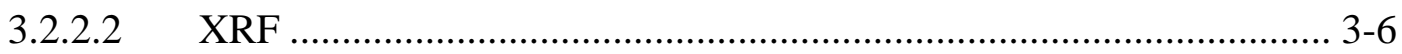

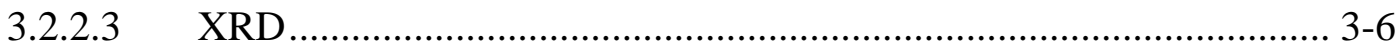

3.3 Lab Methodologies............................................................................ 3-7

3.3.1 General Methodologies …............................................................ 3-7 
3.3.1.1 Stock Solution............................................................................... 3-7

3.3.1.2 pH Measurement......................................................................... 3-9

3.3.1.3 Turbidity Measurement …................................................................. 3-9

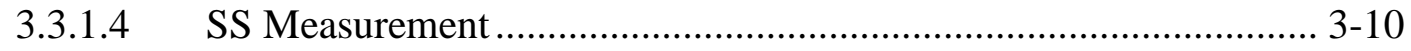

3.3.1.5 UV-Vis Measurement .............................................................. 3-10

3.3.1.6 ICP-MS Measurement ........................................................ 3-11

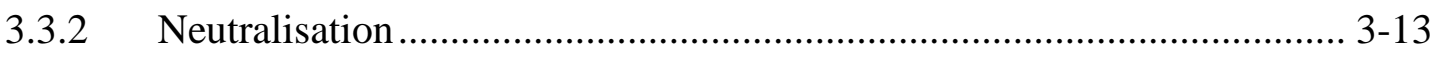

3.3.3 Jar Testing ............................................................................. 3-14

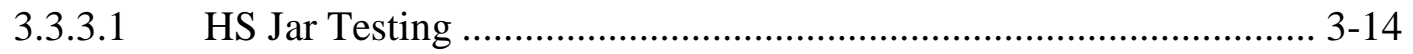

3.3.3.2 MSS Polymer Mixing Conditions .............................................. 3-17

3.3.4 Supernatant Sampling and Analysis .................................................... 3-19

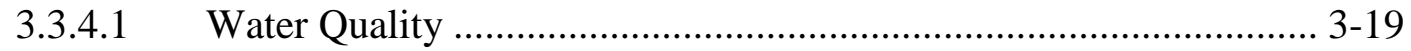

3.3.4.2 Metal Removal ....................................................................... 3-20

3.3.5 Speciation Modelling ....................................................................... 3-21

3.3.5.1 Experimental Speciation ...................................................... 3-21

3.3.5.2 Theoretical Speciation .......................................................... 3-22

Chapter 4. Material Characterisation ............................................................... 4-1

4.1 Humic Substances ......................................................................... 4-1

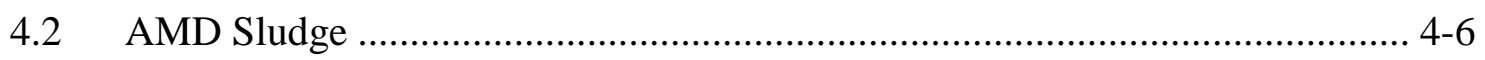

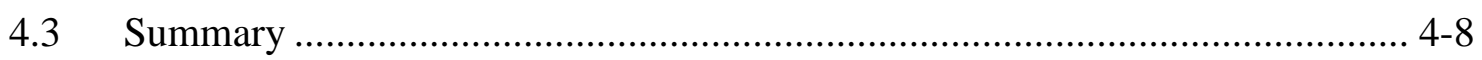

Chapter 5. Neutralisation Dose ........................................................................... 5-1

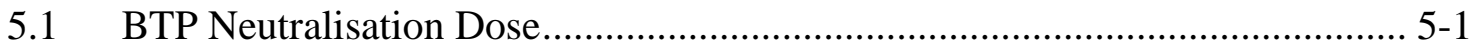

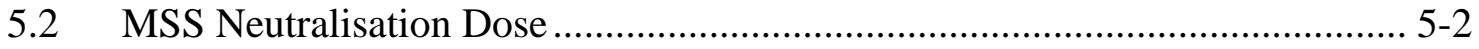

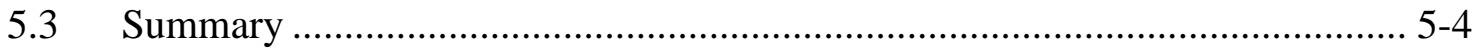

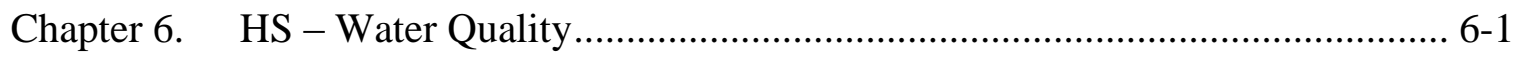

6.2 HS Jar Testing Water Quality ............................................................. 6-2

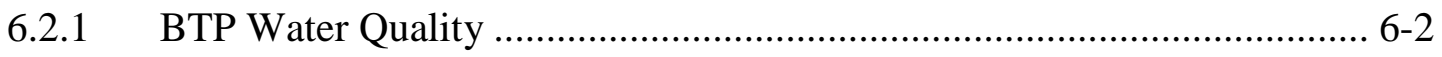




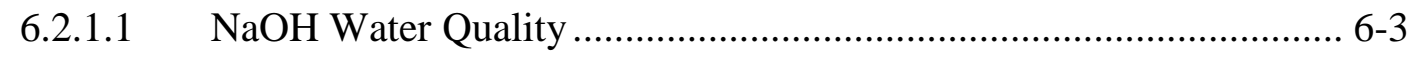

6.2.1.2 $\mathrm{Ca}(\mathrm{OH})_{2}$ Water Quality ............................................................... 6-7

6.2.2 MSS Water Quality .............................................................................. 6-10

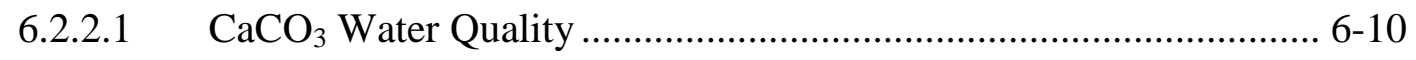

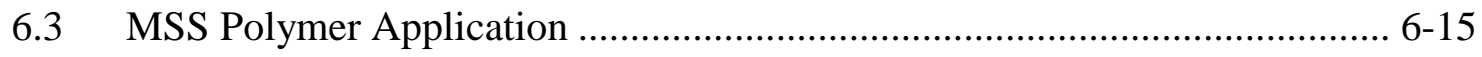

6.3.1 SEHA Water Quality ...................................................................... 6-15

6.3.2 Crystalfloc Polymer Comparison.......................................................... 6-18

6.3.2.1 Crystalfloc B570 Polymer ................................................................ 6-18

6.3.2.2 Crystalfloc B610 Polymer ................................................................. 6-20

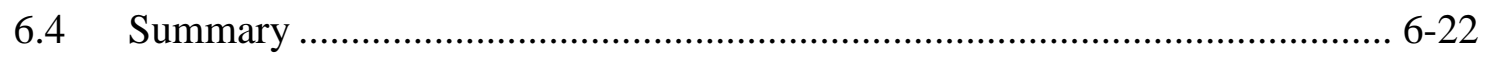

Chapter 7. HS - Metal Removal............................................................................... 7-1

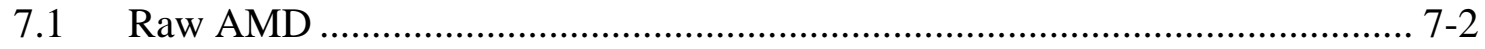

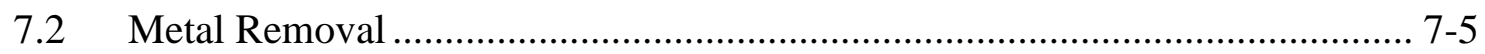

7.2.1 BTP Metal Removal ....................................................................... 7-6

7.2.1.1 $\quad \mathrm{NaOH}$ Metal Removal ...................................................................... 7-7

7.2.1.2 $\quad \mathrm{Ca}(\mathrm{OH})_{2}$ Metal Removal ............................................................ 7-13

7.2.2 MSS Metal Removal......................................................................... 7-20

7.2.2.1 $\quad \mathrm{CaCO}_{3}$ Metal Removal ............................................................. 7-20

7.3 Equilibrium Speciation............................................................................ 7-26

7.3.1 BTP NaOH Equilibrium Speciation Modelling …………………………. 7-27

7.3.1.1 $\mathrm{NaOH}$ Experimental Speciation Modelling ....................................... 7-27

7.3.1.2 $\mathrm{NaOH}$ Theoretical Speciation Modelling ………………………….. 7-28

7.3.2 $\quad \mathrm{BTP} \mathrm{Ca}(\mathrm{OH})_{2}$ Equilibrium Speciation Modelling .................................. 7-31

7.3.2.1 $\quad \mathrm{Ca}(\mathrm{OH})_{2}$ Experimental Speciation Modelling ................................. 7-31

7.3.2.2 $\mathrm{Ca}(\mathrm{OH})_{2}$ Theoretical Speciation Modelling ……........................... 7-32

7.3.3 $\mathrm{MSS}^{2} \mathrm{CaCO}_{3}$ Equilibrium Speciation Modelling ……………….............. 7-35

7.3.3.1 $\quad \mathrm{CaCO}_{3}$ Experimental Speciation Modelling .................................... 7-35 


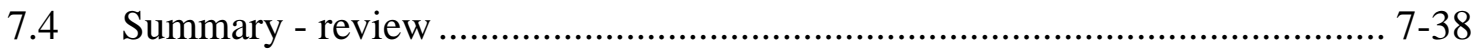

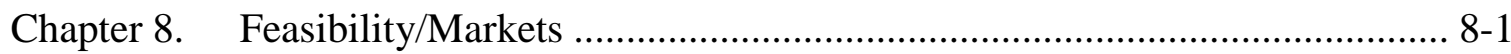

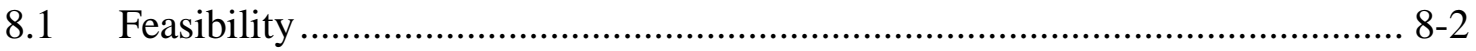

8.1.1 Cost: benefit of HS as a Polymer...................................................... 8-2

8.1.2 Cost: benefit of HS for Metal Removal .................................................. 8-4

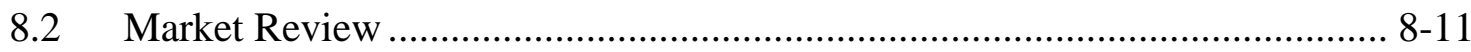

8.2.1 Agricultural Markets ......................................................................... 8-11

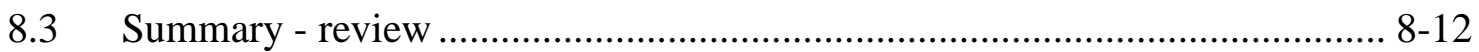

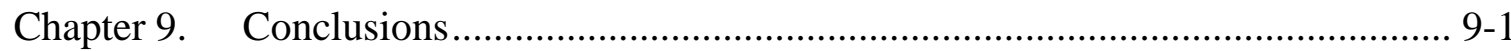

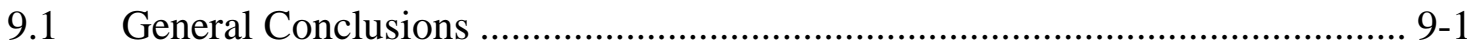

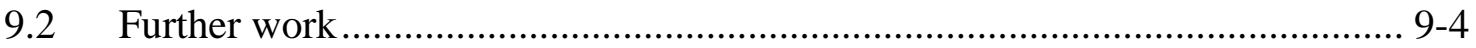

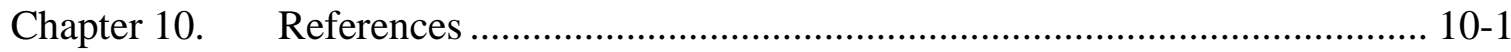

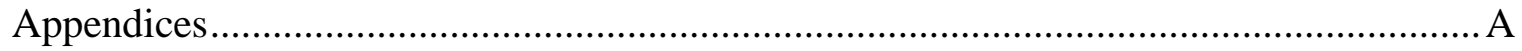

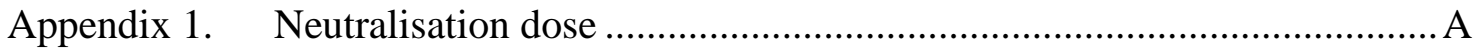

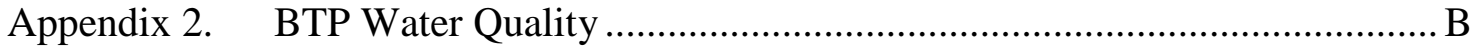

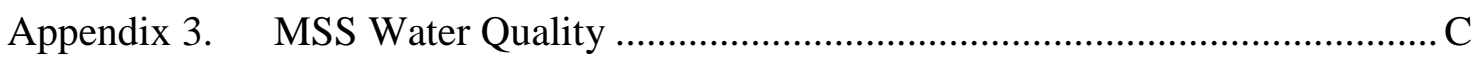

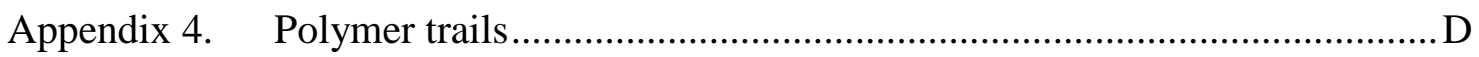

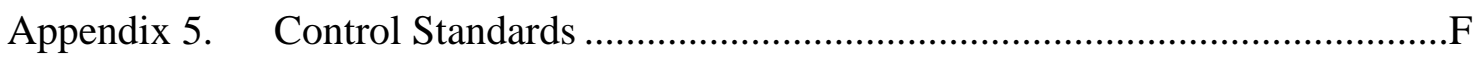

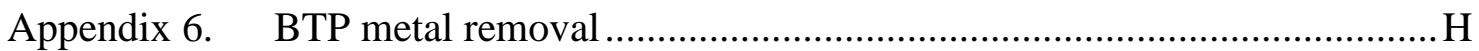




\section{List of Figures}

Figure 2-1: Destabilisation and flocculation of colloidal particles ............................. 2-16

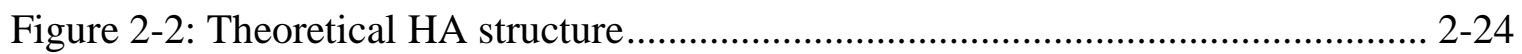

Figure 2-3: pH dependant HA-metal complexation affinity ...................................... 2-30

Figure 2-4: Catechol and hydroquinoic moiety .................................................... 2-33

Figure 3-1: Schematic of relevant Water bodies........................................................ 3-2

Figure 3-2: Calibration curve for UV-Vis measurement of HS concentration .............. 3-11

Figure 3-3: HS Jar Test context ...................................................................... 3-14

Figure 3-4: BTP conditions for $\mathrm{NaOH}$ neutralised AMD ......................................... 3-15

Figure 3-5: Mixing regime for HS dosed BTP scenario ......................................... 3-16

Figure 3-6: Mixing regime for HS dosed MSS scenario .......................................... 3-17

Figure 3-7: Polymer jar test context.................................................................. 3-17

Figure 3-8: Dose conditions for polymer investigation ......................................... 3-18

Figure 3-9: Water quality measurement context................................................ 3-19

Figure 3-10: Metal removal measurement context ............................................... 3-20

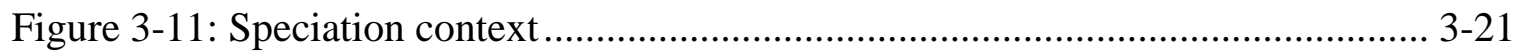

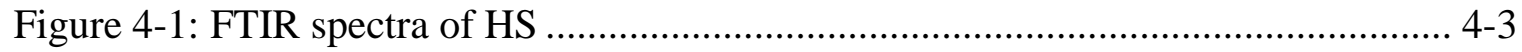

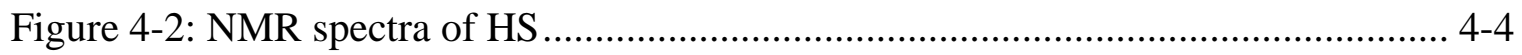

Figure 4-3: FTIR spectra of AMD sludge .................................................................... 4-6

Figure 4-4: XRF composition of AMD sludge …........................................................ 4-7

Figure 5-1: BTP neutralisation pathway ….............................................................. 5-1

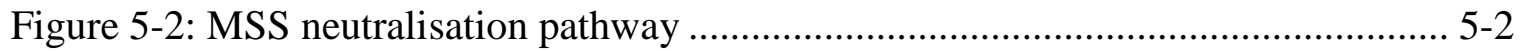

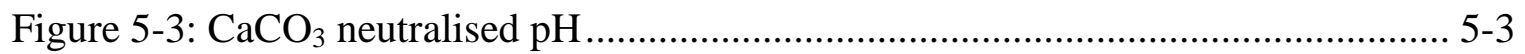

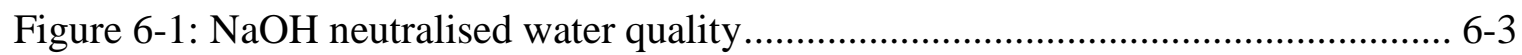


Figure 6-2: $\mathrm{NaOH}$ neutralised supernatant (L to R: SEHA20, SEHA100, AHA20)...... 6-5

Figure 6-3: Comparison of $\mathrm{NaOH}$ neutralised SEHA20 sludge ................................... 6-5

Figure 6-4: $\mathrm{Ca}(\mathrm{OH})_{2}$ neutralised water quality ...................................................... 6-7

Figure 6-5: $\mathrm{Ca}(\mathrm{OH})_{2}$ neutralised supernatant (L to R: SEHA20, SEHA100, AHA20) .. 6-8

Figure 6-6: Comparison of $\mathrm{Ca}(\mathrm{OH})_{2}$ neutralised SEHA20 sludge ................................ 6-8

Figure 6-7: $\mathrm{CaCO}_{3}$ neutralised water quality ...................................................... 6-10

Figure 6-8: $\mathrm{CaCO}_{3}$ neutralised supernatant (L to R: SEHA20, SEHA100, AHA20) ... 6-12

Figure 6-9: Comparison of $\mathrm{CaCO}_{3}$ neutralised SEHA20 sludge ................................ 6-12

Figure 6-10: HS polymer effect on water quality ................................................. 6-15

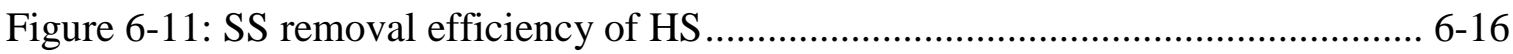

Figure 6-12: B570 polymer effect on water quality............................................... 6-18

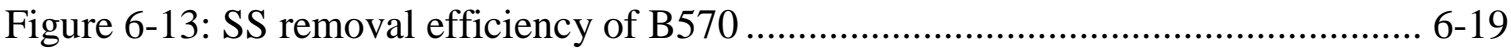

Figure 6-14: B610 polymer effect on water quality................................................ 6-20

Figure 6-15: SS removal efficiency of B610 ............................................................ 6-21

Figure 7-1: Raw AMD concentration ……….......................................................... 7-3

Figure 7-2: $\mathrm{NaOH}$ neutralised control standards .................................................... 7-7

Figure 7-3: $\mathrm{NaOH}$ HS dosed metal concentrations................................................. 7-10

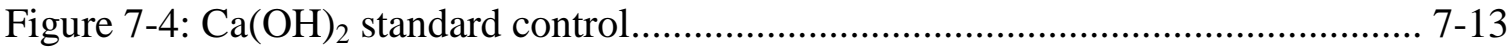

Figure 7-5: Metal concentrations of $\mathrm{Ca}(\mathrm{OH})_{2}$ neutralised AMD.............................. 7-16

Figure 7-6: $\mathrm{CaCO}_{3}$ control standards .......................................................................... 7-20

Figure 7-7: $\mathrm{CaCO}_{3}$ neutralised residual metal concentrations .................................... 7-23

Figure 7-8: $\mathrm{NaOH}$ residual modelled metal speciation................................................. 7-27

Figure 7-9: Theoretical modelled speciation of $\mathrm{NaOH}$ neutralised AMD.................... 7-28

Figure 7-10: Na binding to fulvic and humic electrostatic sites ............................... 7-29

Figure 7-11: $\mathrm{NaOH}$ Fulvic carboxylic and phenolic complexation............................. 7-29

Figure 7-12: $\mathrm{NaOH}$ Humic carboxylic and phenolic complexation ............................ 7-30 
Figure 7-13: $\mathrm{Ca}(\mathrm{OH})_{2}$ residual modelled metal speciation

Figure 7-14: Theoretical modelled speciation of $\mathrm{Ca}(\mathrm{OH})_{2}$ neutralised AMD

Figure 7-15: $\mathrm{Ca}(\mathrm{OH})_{2}$ derived $\mathrm{Ca}$ binding to fulvic and humic electrostatic sites $7-33$

Figure 7-16: $\mathrm{Ca}(\mathrm{OH})_{2}$ fulvic carboxylic and phenolic complexation $7-33$

Figure 7-17: $\mathrm{Ca}(\mathrm{OH})_{2}$ humic carboxylic and phenolic complexation 7-34

Figure 7-18: $\mathrm{CaCO}_{3}$ residual metal speciation.

Figure 7-19: Theoretical modelled speciation of $\mathrm{CaCO}_{3}$ neutralised AMD. 7-36

Figure 7-20: $\mathrm{CaCO}_{3}$ fulvic carboxylic and phenolic complexation 7-37

Figure 7-21: $\mathrm{CaCO}_{3}$ humic carboxylic and phenolic complexation 7-37

Figure 8-1: HS and polymer doses for cost benefit $8-2$

Figure 8-2: Distribution of HS dose cost $8-5$

Figure 8-3: $\mathrm{NaOH}$ neutralised metal removal cost: benefit $8-6$

Figure 8-4: $\mathrm{Ca}(\mathrm{OH})_{2}$ neutralised metal removal cost: benefit $8-8$ 


\section{List of Tables}

Table 1-1: Expected dose order influence on water quality and metal removal............. 1-3

Table 2-1: Environmental occurrence of the target metal group ................................... 2-4

Table 2-2: Variability of Stockton AMD .................................................................... 2-5

Table 2-3: AMD metal concentrations for the headwaters of the Mangatini Stream ...... 2-6

Table 2-4: Target $\mathrm{pH}$ for rapid removal of various metals as hydroxides .................... 2-10

Table 2-5: Classification of Lewis Acids and Bases ................................................... 2-11

Table 2-6: Alkalinity reagents used in AMD neutralisation....................................... 2-14

Table 2-7: Factors affecting selection of alkalinity reagent for AMD neutralisation.... 2-14

Table 2-8: AZNECC guideline trigger values for target metals ................................ 2-21

Table 2-9: HS classification based on acid/alkali solubility ..................................... 2-24

Table 2-10: Physical and chemical properties of humic and fulvic acids.................... 2-25

Table 2-11: Important HS functional groups ....................................................... 2-26

Table 2-12: Dissociation constants of HA and FA functional groups $\left(\log \mathrm{K}_{\mathrm{i}}\right) \ldots \ldots \ldots \ldots \ldots . .2-27$

Table 2-13: Stability constants for HS complexation by functional group .................. 2-28

Table 2-14: Complexation hierarchy of functional groups ........................................ 2-29

Table 3-1: Detection limits of target metals for this thesis ..................................... 3-12

Table 4-1: Elemental mass composition of HS as a percentage .................................. 4-1

Table 4-2: Distribution of carbon in HS structural groups ......................................... 4-5

Table 4-3: Mineralogy of AMD sludge …............................................................. 4-8

Table 7-1: Thesis metal detection limits .................................................................. 7-4

Table 7-2: $\mathrm{NaOH}$ neutralised control standard trend lines and residuals ....................... 7-8

Table 7-3: $\mathrm{Ca}(\mathrm{OH})_{2}$ neutralised control standard trend lines and error ........................ 7-14

Table 7-4: BTP level of protection of treated AMD .................................................. 7-18 
Table 7-5: $\mathrm{Ca}(\mathrm{OH})_{3}$ neutralised control standard trend lines and error

Table 7-6: MSS level of protection of treated AMD $7-25$

Table 7-7: HS binding sites. $7-26$

Table 8-1: Cost: benefit for polymer application...... 8-3

Table 8-2: Required HS dosed metal concentrations $(\mathrm{NaOH})$ $8-7$

Table 8-3: Required HS dosed metal concentrations $\left(\mathrm{Ca}(\mathrm{OH})_{2}\right)$ $8-9$ 


\section{Acknowledgements}

The author would like to acknowledge and express his gratitude to the following people who have significantly contributed to this Master of Engineering Thesis.

My supervisors, Dr. Daniel Tsang and Dr. Paul Weber, have had immensely valuable input into the design of experiments, interpretation of data, and preparation and review of the thesis. They provided me with an academic research project which was highly relevant to real world engineering applications.

The University of Canterbury technical staff, particularly Peter McGuigan and Dave McPherson, have always been willing to help resolve any issues which arose in the lab. Their input especially helped develop the methods of water quality analysis.

The University of Canterbury Chemistry Department, particularly Dr. Sally Gaw and Robert Stainthorpe, made the volume of ICP-MS analysis required by this project possible, despite significant earthquake interruptions. Their instruction greatly improved my understanding of dissolved metal analysis.

I would also like to thank my friends and family, for supporting my commitment to this project. I look forward to spending weekends in your company again!

This project was generously funded by the Foundation for Research, Science and Technology, Solid Energy New Zealand Ltd, and the University of Canterbury Civil and Natural Resource Engineering Department. Without all the parties involved, this research would not have been possible. 


\section{Abstract}

Addition of alkalinity to acid mine drainage (AMD) results in the neutralisation of acidity and precipitation of dissolved metals as insoluble hydroxides. Two aspects of the current AMD treatment practice at the Stockton Mine could be enhanced. Firstly, residual water quality may be poor due to unreacted alkalinity reagents, particularly $\mathrm{CaCO}_{3}$, resulting in poor water clarity and elevated suspended solids (SS). Secondly, neutralisation to circumneutral $\mathrm{pH}$ may not avoid the discharge of residual metals ( $\mathrm{Ni}$ and $\mathrm{Zn}$ ) due to incomplete adsorption and hydroxide precipitation.

The aim of this project was to enhance the conventional neutralisation of AMD through addition of humic substances (HS) to reduce residual SS and trace metal concentrations. Humic substances are organically derived and have a high molecular weight. Functional groups on the surface of HS are capable of binding dissolved metals, forming HS-metal complexes. Incorporation of HS complexed metals into settling floc could result in increased metal removal from the dissolved phase.

Modified jar testing was used to investigate the effects of HS addition before, after and during ( $\mathrm{pH}$ 4.5) neutralisation of AMD in two treatment scenarios at the Stockton Mine; the Blackwater Treatment Plant (BTP) using $\mathrm{NaOH}$ and $\mathrm{Ca}(\mathrm{OH})_{2}$ and the Mangatini Stream-sump System (MSS) using $\mathrm{CaCO}_{3}$. Supernatant samples collected during the sedimentation period were analysed for basic water quality parameters (turbidity and suspended solids) and dissolved $(<0.45 \mu \mathrm{m})$ metal concentrations.

The addition of HS to the BTP process before ( $\mathrm{pH} 2.8$ ) and during ( $\mathrm{pH} 4.5$ ) neutralisation resulted in HS precipitation. Precipitated HS subsequently acted as a nucleation site, triggering flocculation of precipitating metal hydroxides, resulting in low turbidity and suspended solids (SS) of less than $2 \mathrm{NTU}$ and $5 \mathrm{mg} / \mathrm{L}$, respectively. The addition of HS after neutralisation ( $\mathrm{pH}$ 7) did not result in HS precipitation. Intermolecular bridging of HS by the divalent $\mathrm{Ca}$ resulted in incorporation of HS into floc when neutralised by $\mathrm{Ca}(\mathrm{OH})_{2}$, resulting in low turbidity and SS. However, in $\mathrm{NaOH}$ neutralised conditions, the monovalent $\mathrm{Na}$ was unable to bridge $\mathrm{HS}$ molecules, resulting in $\mathrm{HS}$ remaining dissolved and contributing to elevated turbidity and SS of up to 24.4 NTU and 18.4, respectively. 
The neutralisation efficiency of $\mathrm{CaCO}_{3}$ is relatively low, thus approximately $1000 \mathrm{mg} / \mathrm{L}$ $\mathrm{CaCO}_{3}$ remained unreacted in MSS scenarios, resulting in elevated turbidity and SS. When added after neutralisation, dissolved Solid Energy Humic Acid (SEHA) facilitated flocculation of residual $\mathrm{CaCO}_{3} \mathrm{SS}$, resulting in an up to $75 \%$ lower suspended solids than $\mathrm{CaCO}_{3}$ neutralisation alone conditions. Although the results are good, the efficiency of SEHA as a polymer compared unfavorably in a cost: benefit analysis to two commercially available polymers for the removal of residual $\mathrm{CaCO}_{3}$.

Neutralisation of AMD in control samples resulted in decreased concentrations of the target metal group $(\mathrm{Ni}, \mathrm{Zn}, \mathrm{Cu}, \mathrm{Cd}$, and $\mathrm{Pb}$ ) by hydroxide precipitation, co-precipitation, and adsorption. Equilibrium speciation modeling showed that the HS-metal binding affinity controlled the effectiveness of HS addition for metal removal. The low HS complexation affinity of $\mathrm{Ni}$ and $\mathrm{Zn}$ resulted in no additional metal removal by HS dosing. The removal of $\mathrm{Cu}$ was enhanced by over $50 \%$ for SEHA 20 during-neutralisation conditions neutralised by both $\mathrm{NaOH}$ and $\mathrm{Ca}(\mathrm{OH})_{2}$. Up to $80 \%$ lower $\mathrm{Cd}$ concentrations were observed for all HS dose conditions when neutralised by $\mathrm{Ca}(\mathrm{OH})_{2}$. Data for $\mathrm{CaCO}_{3}$ HS dosed metal removal was statistically indeterminate. The high detection limit for $\mathrm{Pb}$ made any HS dosed removal enhancement difficult to identify, which was unfortunate as $\mathrm{Pb}$ has a high HS complexation affinity (Čežíková, Kozler et al. 2001; Milne, Kinniburgh et al. 2003). A simple cost: benefit analysis showed that the additional removal of metals by HS dosing was less efficient than conventional neutralisation alone, on a cost basis.

Overall, incorporation of HS into AMD treatment results in improved water quality for $\mathrm{CaCO}_{3}$ neutralisation and lower concentrations of metals with a high HS binding affinity, for some conditions. However, further investigation is required to improve the feasibility of HS incorporation into the AMD neutralisation process. 


\section{Chapter 1. Introduction}

The adverse environmental impacts of mining represent a significant liability to both society and mine operators. Management of environmental impacts is therefore critical to the economic viability of mining companies. Cost effective reduction of these liabilities may result in better financial and environmental performance for the mine operator, while continuation of a legacy of poor environmental management may reduce the chances of new mine development, due to adverse stakeholder sentiment.

Acid mine drainage (AMD) is the most serious potential environmental impact at coal mines which disturb pyritic overburden on the West Coast of the South Island of New Zealand (Pope, Newman et al. 2010a). Acid mine drainage is characterised by high acidity and dissolved metal (Fe, Al, Ni, Zn) concentrations (Black, Trumm et al. 2005). The number of taxa present in AMD impacted streams is negatively correlated to $\mathrm{pH}$ (acidity), Fe, and Al concentrations (Winterbourn, McDiffett et al. 2000; Harbrow 2001).

Treatment of AMD by neutralisation involves dosing with alkalinity to increase $\mathrm{pH}$ and trigger hydroxide precipitation of metals (Skousen, Sexton et al. 2000). Metals are removed by direct precipitation, co-precipitation, or adsorption to hydroxide precipitates resulting in decreased supernatant concentrations (Webster, Swedlund et al. 1998; Younger, Banwart et al. 2002). Often, discharge water quality criteria is based on the ANZECC guidelines (ANZECC 2000), however, site specific criteria may be developed for each mine site, stating limits for metal concentration and water clarity; e.g., the SENZ Cypress Project water quality is based on the work of Grieg et al. (2010).

At the SENZ Stockton Mine, treatment of AMD is undertaken using $\mathrm{Ca}(\mathrm{OH})_{2}$ and ultrafine $(90 \mathrm{wt} \%$ particles less than $106 \mu \mathrm{m})$ limestone $\left(\mathrm{CaCO}_{3}\right)$ neutralisation. Two aspects of the current AMD treatment practise at the Stockton Mine could be enhanced. Firstly, residual water quality may be poor due to unreacted alkalinity reagent, particularly $\mathrm{CaCO}_{3}$, resulting in poor water clarity and elevated suspended solids (SS). Secondly, neutralisation to circum-neutral $\mathrm{pH}$ may not avoid the discharge of residual metals $(\mathrm{Ni}$ and $\mathrm{Zn}$ ) due to incomplete adsorption to $\mathrm{Fe}$ hydroxides and hydroxide precipitation. 
Introduction

\subsection{Statement of the Problem}

The quality of water draining mine sites is degraded by suspended sediment, environmentally significant trace elements, and acidity (Pope, Newman et al. 2010a). The main goal of AMD neutralisation and treatment is to raise the $\mathrm{pH}$ and lower metal concentrations by forming insoluble metal hydroxides/precipitates (Trumm 2010).

Incomplete dissolution of some alkalinity reagents, particularly $\mathrm{CaCO}_{3}$, may result in elevated turbidity and SS in discharge waters (Davies, Weber et al. 2011a), potentially violating discharge limits.

When neutralised to $\mathrm{pH}$ 6-7, hydroxide precipitation of some metals, including $\mathrm{Ni}, \mathrm{Zn}$, and $\mathrm{Cd}$ is incomplete (Kelly 1988), resulting in discharge of potentially harmful metals. Both $\mathrm{Pb}$ and $\mathrm{Cu}$ have a relatively high sorption affinity for hydroxide precipitates (Lee, Bigham et al. 2002) and may be partially removed during neutralisation.

\subsection{Purpose of the Study}

This Masters of Engineering research project aims to investigate the potential for humic substance (HS) incorporation into conventional AMD neutralisation. Humic substances have the potential to from HS-metal complexes, depending on specific metal-HS complexation affinities (Milne, Kinniburgh et al. 2003). Incorporation of HS complexed metals into settling floc may increase the removal of the target metal group $(\mathrm{Ni}, \mathrm{Zn}, \mathrm{Cu}$, $\mathrm{Cd}$, and $\mathrm{Pb}$ ) during $\mathrm{AMD}$ neutralisation. The following research questions were used to identify under which conditions the treatment of AMD is enhanced by addition of HS.

- How does HS enhancement of the conventional AMD neutralisation process affect the residual supernatant water quality in terms of SS and turbidity?

- How does HS enhancement of the conventional AMD neutralisation process affect the residual concentration of metals?

The experimental design aimed to simulate a range of possible scenarios for $\mathrm{HS}$ incorporation into AMD neutralisation. Thus, a range of alkalinity reagents $(\mathrm{NaOH}$, $\mathrm{Ca}(\mathrm{OH})_{2}$ and $\left.\mathrm{CaCO}_{3}\right)$ and humic substances (lignite extracted Solid Energy Humic Acid (SEHA) and Aldrich Humic Acid (AHA)) and were used in lab trails.

Three dose orders were used to determine the optimal HS addition scenario with respect to water quality and metal removal; addition of HS before-, after- and during- 
Introduction

neutralisation. The expected degree of flocculation and metal binding competition on water quality and metal removal for each dose scenario is shown in Table 1-1.

Table 1-1: Expected dose order influence on water quality and metal removal

\begin{tabular}{ccc}
\hline & Flocculation - Water quality & Binding competition - Metal removal \\
\hline Before & High & Low \\
After & Low & High \\
during & Moderate & Moderate \\
\hline
\end{tabular}


Introduction

\subsection{Significance to the Field}

There are extensive publications which describe AMD generation and the corresponding impacts on the receiving environment, both in New Zealand and internationally (section 2.1). Neutralisation of AMD by conventional methods and metal removal processes including hydroxide precipitation, co-precipitation and adsorption are also relatively well researched (section 2.2). The specific properties of HS responsible for the formation of metal-HS complexes in naturally humic aquatic and artificial lab environments have also been investigated and quantified (section 2.3).

There is however limited literature of HS in engineering type applications for removal of dissolved metals (section 2.3.3). Of the existing publications reviewed for this thesis, none used alkali extracted $(\mathrm{pH} \sim 10-12)$ liquid phase HS for metal recovery or used raw AMD as a stock metal solution.

This thesis is significant as it aims to apply HS to the engineered treatment of AMD. The initial deprotonation of HS functional groups at alkali $\mathrm{pH}$ theoretically enhances the capacity for neutralisation of acidity and complexation of dissolved metals. Thus, use of a liquid HS at alkali $\mathrm{pH}$ would maximise the potential mixing and reactivity of HS dosing.

The majority of the HS-metal complexation capacity data published in the literature are for simple solutions of HS, one metal, and background ions. The complex chemistry of raw AMD introduces a range of phenomena, which may enhance or inhibit the complexation of metals by HS. These phenomena may include binding competition from other metals/electrolytes for HS complexation sites, adsorption to metal hydroxide precipitates $\left(\mathrm{Fe}(\mathrm{OH})_{3}, \mathrm{Al}(\mathrm{OH})_{3}\right)$ and other mineral sites, and complexation with nonorganic ligands (i.e. $\mathrm{SO}_{4}{ }^{2-}$ ). Thus, the use of HS complexation affinities for metals quoted from the literature may be unreliable for AMD applications. 


\section{Chapter 2. Literature Review}

The literature review is divided into four sections

Section 1: Background. This section describes the process of acid mine drainage (AMD) generation and identifies key factors which influence AMD chemistry in general and at the Solid Energy New Zealand Ltd (SENZ) Stockton Coal Mine. The impact of AMD on biota in the immediate and downstream reach is then discussed and key factors which influence the toxicity of metals to biota are identified.

Section 2: Standard methods of AMD treatment. This section describes the removal of metals from AMD by neutralisation. The incorporation of neutralisation techniques in active and passive AMD treatment is then described.

Section 3: HS for AMD treatment. This section describes humic substance (HS) properties and metal complexation phenomena. Previous work using HS in treatment of metal contaminated waters is summarised.

Section 4: Lignite-derived HS for AMD remediation. This section briefly states the case for HS application to AMD remediation and identifies the gap in the literature this thesis aims to fill.

The term Humic Substances (HS) is used throughout this chapter and refers to the alkali extractable humic acid (HA) and fulvic acid (FA) fraction of HS. 


\subsection{Background - AMD Evolution and Toxicity}

This section discusses the generation of AMD and factors which affect the resulting acidity and metal concentration. The impact of AMD on receiving aquatic ecosystems is then discussed and any mechanisms that reduce the observed toxicity are identified.

\subsubsection{AMD}

Pope et al. (2010a) list 12 publications which state that AMD is the most serious potential environmental impact at coal mines on the West Coast of the South Island of New Zealand. During mine operation AMD may be generated from mine pits, exposed coal seams, mine facilities, disturbed overburden material, coal stockpiles, access roads, ditches and underground walls, ceilings and floors (Trumm 2010). The chemistry of AMD varies, but the main properties are high acidity and elevated metal concentrations.

\subsubsection{Pyrite Oxidation}

Deposition of the Brunner Coal Measures (BCM) occurred in a fluvial to estuarine environment (Pope, Newman et al. 2010a), which provided a continuous supply of sulphate, iron and decomposable organic matter conducive to pyrite formation (Evangelou 1995). Mudstones and siltstones in BCM overburden tend to be elevated in pyrite (Weisener and Weber 2010). When exposed to the atmosphere (oxygen and water), iron sulphide compounds may be oxidised, producing acidity. Oxidation of pyrite $\left(\mathrm{FeS}_{2}\right)$ occurs according to Equation 2-1 to Equation 2-3.

$$
\mathrm{FeS}_{2(\mathrm{~s})}+3.5 \mathrm{O}_{2(\mathrm{~g})}+\mathrm{H}_{2} \mathrm{O}_{(\mathrm{l})} \rightarrow \mathrm{Fe}^{2+}+2 \mathrm{SO}_{4}^{2+}+2 \mathrm{H}^{+} \quad \text { Equation 2-1 }
$$

Oxidation of reduced $\mathrm{S}$ to $\mathrm{SO}_{4}{ }^{2-}$

$$
\mathrm{Fe}^{2+}+0.25 \mathrm{O}_{2}+\mathrm{H}^{+} \rightarrow \mathrm{Fe}^{3+}+0.5 \mathrm{H}_{2} \mathrm{O}_{(l)}
$$

Equation 2-2

Oxidation of $\mathrm{Fe}$ (II) to $\mathrm{Fe}(\mathrm{III})$

$$
\mathrm{Fe}^{3+}+3 \mathrm{H}_{2} \mathrm{O}_{(l)} \leftrightarrow \mathrm{Fe}(\mathrm{OH})_{3(s)}+3 \mathrm{H}^{+}
$$

Hydrolysis of $\mathrm{Fe}(\mathrm{III})$ to precipitate $\mathrm{Fe}(\mathrm{OH})_{3(\mathrm{~s})}$

$$
\mathrm{FeS}_{2(\mathrm{~s})}+7 / 2 \mathrm{H}_{2} \mathrm{O}+15 / 4 \mathrm{O}_{2} \rightarrow \mathrm{Fe}(\mathrm{OH})_{3(\mathrm{~s})}+2 \mathrm{SO}_{4}^{2-}+4 \mathrm{H}^{+}
$$

Complete oxidation and hydrolysis reaction

(Evangelou 1995; Pope, Newman et al. 2010a) 
Equation 2-4 shows that the overall reaction produces four moles of acidity for every mole of pyrite oxidised. The $\mathrm{Fe}^{3+}$ produced in Equation 2-2 may directly oxidise pyrite in a parallel acid producing reaction.

$$
\begin{gathered}
\mathrm{FeS}_{2(s)}+14 \mathrm{Fe}^{3+}+8 \mathrm{H}_{2} \mathrm{O} \rightarrow 15 \mathrm{Fe}^{2+}+2 \mathrm{SO}_{4}^{2-}+16 \mathrm{H}^{+} \\
\mathrm{Fe}^{3+} \text { oxidation of Pyrite }
\end{gathered}
$$

(Evangelou 1995; Younger, Banwart et al. 2002)

Equation 2-5 also produces four moles of acid per mole of pyrite, after $\mathrm{Fe}^{3+}$ hydrolysis, provided there is sufficient dissolved oxygen to facilitate Equation 2-2. The oxidation of pyrite by $\mathrm{Fe}^{3+}$ is the dominant reaction in the formation of AMD from $\mathrm{pH}$ 2-9; while the role of oxygen in pyrite oxidation is to facilitate Equation 2-2 (Moses, Kirk Nordstrom et al. 1987).

The rate of abiotic pyrite oxidation is limited by Equation 2-2, the oxidation of $\mathrm{Fe}^{2+}$ to $\mathrm{Fe}^{3+}$ (Evangelou 1995). However, at low pH, Equation 2-2 is catalysed by acidophilic bacteria (Younger, Banwart et al. 2002). The rate of pyrite oxidation remains high provided sufficient $\mathrm{Fe}^{3+}$ and dissolved oxygen; however, as $\mathrm{Fe}^{2+}$ and $\mathrm{H}^{+}$accumulate the rate of pyrite oxidation decreases (Williamson and Rimstidt 1994; Holmes and Crundwell 2000). Thus, acidity accumulates in the system until Equation 2-3 reaches equilibrium due to a high $\mathrm{H}^{+}$concentration, resulting in AMD with a high Fe concentration; or $\mathrm{Al}$ concentration if Equation 2-6 occurs.

Pyrite oxidation is a surface controlled reaction. Therefore, pyrite oxidation is accelerated when acid producing material is disturbed, causing an increase in specific surface area. The most reactive form of pyrite is framboidal (Evangelou 1995). Weisner and Weber (2010) differentiated the oxidation of pyrite from euhedral and framboidal morphologies. Oxidation of framboidal pyrite occurred 2-3 times faster than euhedral pyrite, contributing much of the early generation of acidity while residual acidity was generated from euhedral pyrite.

The $\mathrm{H}^{+}$acidity generated can contribute to the dissolution of other minerals and result in the release of acid $(\mathrm{Fe}, \mathrm{Al}, \mathrm{Mn})$, alkali $(\mathrm{Na}, \mathrm{K})$ or alkaline $(\mathrm{Mg}, \mathrm{Ca})$ earth metals. Acid metals contribute to the total acidity of AMD as they undergo hydrolysis below circumneutral $\mathrm{pH}$, which is typically the end point of AMD neutralisation. 
Aluminium is a common acid metal evolved from the dissolution of feldspar, by Equation 2-6.

$$
\begin{aligned}
& \mathrm{KAlSi}_{3} \mathrm{O}_{8(\mathrm{~s})}+10 \mathrm{H}_{2} \mathrm{O}+3 \mathrm{Fe}^{3+}+2 \mathrm{SO}_{4}^{2-} \\
& =\mathrm{KFe}_{3}\left(\mathrm{SO}_{4}\right)_{2} \cdot(\mathrm{OH})_{6(\mathrm{~s})}+3 \mathrm{H}_{4} \mathrm{SiO}_{4}^{0}+2 \mathrm{H}^{+}+\mathrm{Al}^{3+}
\end{aligned}
$$

(Pope, Newman et al. 2010a)

Hydrolysis of $\mathrm{Al}^{3+}$ occurs through a similar reaction to Equation 2-3 (for $\mathrm{Fe}^{3+}$ ) at around $\mathrm{pH} 4.5$, producing further acidity. Hence, both $\mathrm{Fe}$ and $\mathrm{Al}$ contribute to total acidity below circum-neutral $\mathrm{pH}$.

\subsubsection{AMD Variability}

Rock mineralogy is the major factor effecting AMD chemistry (Pope, Weber et al. 2010b). Climate, hydrogeology, and mine type also influence mine drainage chemistry (Pope, Newman et al. 2010a). Depending on the local mineralogy, a range of trace elements may be present. Table 2-1 shows the key mineral sources of the metals discussed in this thesis.

\section{Table 2-1: Environmental occurrence of the target metal group}

\begin{tabular}{cl}
\hline Metal & Mineral trace metal source \\
\hline $\mathbf{N i}$ & Sulphides, serpentines \\
$\mathbf{Z n}$ & Sulphides, oxides \\
$\mathbf{C u}$ & Sulphides, sulphates, sulphosalts, carbonates, and native metal \\
$\mathbf{C d}$ & Complex oxides, sulphides and carbonates in Zinc, Lead, and copper ores \\
$\mathbf{P b}$ & Sulphides, carbonates, sulphates
\end{tabular}

(Ward 2002; Finkelman 2005)

Oxidation of these minerals results in the release of metals, as shown in Equation 2-7 for the oxidation of Sphalerite.

$$
\mathrm{ZnS}_{2(s)}+2 \mathrm{O}_{2(a q)} \rightarrow \mathrm{Zn}^{2+}+\mathrm{SO}_{4}^{2-}
$$

Oxidation of Sphalerite

(Younger, Banwart et al. 2002) 
A number of other metal (As, B, Co, Cd, Hg, etc.) containing minerals exist in coal seams which contribute a range of metals to AMD. The metals released from these minerals are discussed in Moore et al. (2005) and have been excluded from the scope of this thesis.

Trace element concentration is not regulated by hydrolysis at the $\mathrm{pH}$ of AMD (Pope, Newman et al. 2010a), resulting in trace metal accumulation. At low $\mathrm{pH} \mathrm{Cu}$ and $\mathrm{Pb}$ may be slightly adsorbed to particulates resulting in decreased free metal concentrations, while $\mathrm{Ni}$ and $\mathrm{Zn}$ adsorption is negligible (Pope, Newman et al. 2010a).

\subsubsection{Stockton AMD}

Coal has been mined at the Stockton Mine site since 1908 (SENZ 2011). Coal is currently excavated from the BCM at the Stockton Mine by open cast techniques, resulting in significant disruption of potentially acid forming overburden (Pope, Weber et al. 2010b). Multiple sources of AMD exist at the Stockton Coal Mine, which present as streams and seeps. Table 2-2 shows the variability in $\mathrm{AMD} \mathrm{pH}, \mathrm{Fe}$, and $\mathrm{Al}$ concentrations over 11-12 sample sites taken from the Stockton Mine.

Table 2-2: Variability of Stockton AMD

\begin{tabular}{cccc}
\hline & Min & Median & Max \\
\hline $\mathbf{p H}$ & 2.15 & 3.13 & 4.05 \\
$\mathbf{F e}\left(\mathbf{m g L}^{-1}\right)$ & 0.09 & 5.59 & 1410 \\
$\mathbf{A l}\left(\mathbf{m g L}^{-1}\right)$ & 0.46 & 17.9 & 607 \\
\hline
\end{tabular}

(McCauley, O'Sullivan et al. 2010)

Table 2-2 shows significant variation, by up to five orders of magnitude, in AMD chemistry at different sites across the Stockton Mine. McCauley et al. (2010) noted a strong positive correlation between acidity and metal concentration across the Stockton Mine; which was also observed by Collier et al. (1989) and Winterbourn et al. (2000).

The AMD used in this study was collected from the headwaters of the Mangatini Stream, within the Stockton Mine. Pope et al. (2010a) tested AMD from the headwaters of the Mangatini Stream for a comprehensive suite of metals in 2005. They recorded a pH of 2.61 and the metal concentrations shown in Table 2-3. 
Literature Review

Table 2-3: AMD metal concentrations for the headwaters of the Mangatini Stream

\begin{tabular}{cc}
\hline Metal & $\begin{array}{c}\text { Concentration } \\
(\mathbf{m g} / \mathbf{L})\end{array}$ \\
\hline $\mathbf{F e}$ & 39.7 \\
$\mathbf{A l}$ & 83.7 \\
$\mathbf{M n}$ & 2.46 \\
$\mathbf{N i}$ & 0.497 \\
$\mathbf{Z n}$ & 3.14 \\
$\mathbf{C u}$ & 0.109 \\
$\mathbf{C d}$ & 0.003 \\
$\mathbf{P b}$ & 0.144
\end{tabular}

(Pope, Newman et al. 2010a)

The acidity for the headwaters of the Mangatini Stream was calculated as $699 \mathrm{mg} / \mathrm{L}$ $\mathrm{CaCO}_{3}$, based on the hydrogen and mineral acidity using Equation 2-8.

$$
\text { Acidity }_{\text {calculated }}=50\left(\frac{2 F e^{2+}}{56}+\frac{3 F e^{3+}}{56}+\frac{3 A l}{27}+\frac{2 M n}{55}+1000\left(10^{-p H}\right)\right) \quad \text { Equation 2-8 }
$$

Hydrogen and mineral acidity

(Watzlaf, Schroder et al. 2004)

\subsubsection{Environmental Impact/toxicity}

The adverse impact of AMD on stream biota is primarily due to acidity and metal toxicity. This toxicity may be somewhat ameliorated by water hardness and organic content (dissolved HS). Natural attenuation of AMD results in downstream degradation due to hydroxide precipitates covering the streambed.

\subsubsection{Acidity and Metal Toxicity}

The quality of water draining mine sites is degraded by suspended sediment, environmentally significant trace elements, and acidity (Pope, Newman et al. 2010a). When essential and non-essential elements exceed an organism's optimum and tolerable concentration they become toxic. The specific toxic concentration varies according to population tolerance levels, stage of life cycle, and exposure duration (Harding 2005).

The immediate adverse impact of AMD on stream biota is due to excess acidity and soluble metals (O'Halloran, Cavanagh et al. 2008). The response of biota to elevated 
acidity and metal concentrations seems to be species dependant. Some researchers suggest acidity is the dominant parameter affecting mortality (O'Halloran, Cavanagh et al. 2008), while others correlate metal concentration with mortality (Greig, Niyogi et al. 2010). Vascular plants are usually absent from AMD impacted streams while bryophytes and periphyton may be locally abundant (Winterbourn, McDiffett et al. 2000; Harding and Boothroyd 2004). Some algae are extremely abundant in highly acidified streams (pH 2.7-3.1) downstream of abandoned mine sites (Winterbourn, McDiffett et al. 2000). Overall, the number of taxa present in an AMD impacted stream is negatively correlated to $\mathrm{pH}$ (acidity), conductivity, Fe, and $\mathrm{Al}$ concentrations (Winterbourn, McDiffett et al. 2000; Harbrow 2001).

\subsection{Tolerance}

The toxicity of acidity and dissolved metals is complicated by the development of tolerance. The abundance of fish populations in circum-neutral or naturally acidic reference streams were weakly correlated with acidity and metal concentration (Greig, Niyogi et al. 2010), suggesting tolerance of naturally elevated acidity and metals. The development of acidity and metal tolerance in mayflies sourced from naturally acidic streams resulted in greater survival rates in diluted AMD than mayflies from circumneutral streams (O'Halloran, Cavanagh et al. 2008). Collier et al. (1990) reported 24 of the 37 most widespread insect taxa at sites with $\mathrm{pH}<4.5$ and Winterbourn (1998) reported four families of caddisfly larvae in streams with $\mathrm{pH}<3.6$. The degree of tolerance may be population rather than species dependant, with intraspecific variation in acid tolerance observed across New Zealand (Collier, Ball et al. 1990).

\subsection{Hardness AMD Detoxification}

As hardness ( $\mathrm{Ca}$ and $\mathrm{Mg}$ ) increases, the observed toxicity of dissolved metals decreases. In hard waters a higher proportion of metals form insoluble carbonates or adsorb to carbonate precipitates, reducing metal bioavailability and toxicity (Forstner, Wittmann et al. 1981). The higher cation concentration of hard waters essentially dilutes toxic metals. For a fixed number of gill ion-exchange sites, increased hardness decreases the concentration of toxic metals and thus their uptake by fish (Gundersen, Bustaman et al. 1994; Yim, Kim et al. 2006). The use of hardness modified trigger values for protection of aquatic species reflects the detoxification effects of hardness on metals (ANZECC 2000), discussed further in section2.2.3. 


\section{Literature Review}

Increasing the hardness from 44 to $150 \mathrm{mg} / \mathrm{L} \mathrm{CaCO}_{3}$ resulted in an increase in the median lethal concentration $\left(\mathrm{LC}_{50}\right)$ for $\mathrm{Cd}, \mathrm{Cu}, \mathrm{Zn}$ and $\mathrm{Pb}$ of 25, 66, 77 and $88 \%$, respectively (Yim, Kim et al. 2006). Hyne et al. (2005) also showed that increasing the hardness from 44 to $374 \mathrm{mg} / \mathrm{L} \mathrm{CaCO}_{3}$ decreased $\mathrm{Zn}$ toxicity by $57 \%$, at a slightly more acidic $\mathrm{pH}$ than the study of Yim et al. (2006).

\subsection{HS AMD Detoxification}

The toxicity of $\mathrm{Cu}$ is decreased by dissolved organic matter (DOM) in natural streams. Meador (1991) showed the mortality of Daphnia Magna in water with a $0.075 \mathrm{mg} / \mathrm{L} \mathrm{Cu}$ concentration decreased from 100 to $10 \%$ by addition of $3.8 \mathrm{mg} / \mathrm{L}$ dissolved organic carbon (DOC). Ryan et al. (2004) showed the $\mathrm{LC}_{50}$ of $\mathrm{Cu}$ to larval fathead minnows increased linearly with humic acid (HA), up to $\mathrm{Cu}$ and HA concentrations of 2 and 4 $\mathrm{mg} / \mathrm{L}$, respectively.

Collier and Winterbourn (1987) showed that dissolved Al concentration was strongly correlated with DOM in naturally acidic West Coast streams, and suggested that over $90 \%$ of Al was DOM complexed. Gunderson et al. (1994) showed that increasing the HA concentration from 1.4 to $10.1 \mathrm{mg} / \mathrm{L}$ increased the $\mathrm{LC}_{50}$ of $\mathrm{Al}$ to juvenile rainbow trout from 3.75 to $5.22 \mathrm{mg} / \mathrm{L}$. The addition of a nominal $5 \mathrm{mg} / \mathrm{L}$ HS to a $2.5 \mathrm{mg} / \mathrm{L} \mathrm{Al}$ solution increased the specific growth rate of trout by approximately one order of magnitude.

The review paper of Al-Reasi et al. (2011) showed the majority of DOM toxicity data which supports metal detoxification by HS complexation was obtained by toxicity trials using aquatically derived NOM separated by reverse osmosis. Schwartz et al. (2004) showed that optically darker NOM decreased acute $\mathrm{Cu}$ and $\mathrm{Pb}$ toxicity to a greater degree than optically light NOM. The correlation between optical absorbance and aromacity suggests that heavier HS (more typical of lignite derived HS) will be more effective in metal detoxification than aquatically derived NOM.

\subsubsection{Downstream Impact}

As AMD tributaries feed into larger water bodies, natural attenuation occurs through dilution, neutralisation, buffering, oxidation, hydrolysis, and precipitation (Pope, Newman et al. 2010a). Dilution of AMD decreases acidity, triggering hydrolysis of dissolved metals and resulting in the precipitation of insoluble hydroxides. Precipitation causes significant modification to the streambed by cementing substrata, clogging interstitial spaces and covering biota (Harding and Boothroyd 2004). 


\subsection{Standard Methods of AMD Treatment}

This section discusses the response of acidity and metals to neutralisation of acidity, and identifies the removal mechanisms of metals from AMD. The design of active and passive treatment processes to remove metals from AMD via neutralisation is then discussed.

\subsubsection{Neutralisation}

The fate of metals during the neutralisation process is governed by multiple competing removal mechanisms. Metals may form insoluble hydroxide precipitates at circum-neutral $\mathrm{pH}$, complex with inorganic or organic ligands, become incorporated in the precipitating floc by co-precipitation and adsorption, or remain dissolved.

\subsubsection{Hydroxide Precipitation}

The neutralisation process is outlined below with respect to acidity and polyvalent metals $\left(\mathrm{Me}^{2+/ 3+}\right)$, neutralised by calcium hydroxide $\left(\mathrm{Ca}(\mathrm{OH})_{2}\right)$ which is widely used for $\mathrm{pH}$ adjustment in the water treatment industry (Younger, Banwart et al. 2002).

$$
\mathrm{Ca}(\mathrm{OH})_{2(s)}+2 \mathrm{H}_{(a q)}^{+} \rightarrow \mathrm{Ca}_{(a q)}^{2+}+2 \mathrm{H}_{2} \mathrm{O}_{(l)}
$$

Equation 2-9

Dose with alkalinity

$$
\mathrm{Ca}(\mathrm{OH})_{2(s)}+\mathrm{Me}_{(a q)}^{2+} \rightarrow \mathrm{Me}(\mathrm{OH})_{2(s)}+\mathrm{Ca}_{(a q)}^{2+}
$$

Divalent cation oxidation/precipitation

$$
\begin{gathered}
3 \mathrm{Ca}(\mathrm{OH})_{2(s)}+2 \mathrm{Me}_{(a q)}^{3+} \rightarrow 2 \mathrm{Me}(\mathrm{OH})_{3(s)}+3 \mathrm{Ca}_{(a q)}^{2+} \\
\text { Trivalent cation oxidation/precipitation } \\
\mathrm{Ca}_{(a q)}^{+}+\mathrm{SO}_{4(a q)}^{2-}+2 \mathrm{H}_{2} \mathrm{O}_{(\mathrm{l})} \rightarrow \mathrm{CaSO}_{4} \cdot 2 \mathrm{H}_{2} \mathrm{O}_{(s)} \\
\text { Sulphate removal, gypsum }
\end{gathered}
$$

(Lottermoser 2003)

The aim of neutralisation is to raise the $\mathrm{pH}$ to circum-neutral levels and reduce the acidity toxicity discussed in section 2.1.2.1. The solubility of some metals (particularly Fe, Al) is significantly lower at circum-neutral $\mathrm{pH}$, resulting in rapid precipitation. Other potentially eco-toxic trace metals common in AMD require further increase in $\mathrm{pH}$ to precipitate as metal hydroxides. Table 2-4 shows the target $\mathrm{pH}$ required for rapid removal of various metals as hydroxides. 
Literature Review

Table 2-4: Target pH for rapid removal of various metals as hydroxides

\begin{tabular}{ccc}
\hline Metal & $\mathbf{p H}$ & Stability Constant $^{*}$ \\
\hline $\mathbf{F e}^{3+}$ & $3.5-4.3$ & 39.5 \\
$\mathbf{A l}^{3+}$ & $4.9-5.2$ & 33.5 \\
$\mathbf{P b}^{2+}$ & 6.3 & 15.3 \\
$\mathbf{C u}^{2+}$ & $6.8-7.2$ & 19.3 \\
$\mathbf{Z n}^{2+}$ & $8.2-8.4$ & 15.5 \\
$\mathbf{N i}^{2+}$ & 9.3 & 17.2 \\
$\mathbf{F e}^{2+}$ & 9.5 & 15.1 \\
$\mathbf{C d}^{2+}$ & $9.7-9.8$ & 14.3
\end{tabular}

(Kelly 1988; Younger, Banwart et al. 2002; Harding 2005) * (Morel 1983)

NB: The stability constants shown on Table 2-4 are for the formation of insoluble hydroxide precipitates in solutions with zero ionic strength at $25{ }^{\circ} \mathrm{C}$, and are included for reference.

\subsubsection{Inorganic Complexation}

The general reaction between a metal ion, $M$ and an $i^{\text {th }}$ deprotonated ligand, $\mathrm{L}_{\mathrm{i}}$ is written in Equation 2-13.

$$
M+L_{i} \leftrightarrow M L_{i}
$$

Equation 2-13

Metal-ligand complexation

(Sparks 2003)

The stability constant is the ratio of rate constants for the forward and reverse reactions, and states the concentration of reactant and product species at equilibrium (Snoeyink and Jenkins 1980). The stability constant is therefore:

$$
K_{i}^{\text {cond }}=\frac{\left[M L_{i}\right]}{[M]\left[L_{i}\right]}
$$

Conditional stability constant

(Stevenson 1994; Sparks 2003)

The interaction between metals and inorganic/organic ligands can be predicted based on the hydrolysis properties and hardness/softness of metals and complexing sites (Sparks 2003). The complexation of metals by organic ligands (HS) is discussed in section 2.3.2. 
The hydrolysis properties of metals influence their reaction with the major ligand in water, $\mathrm{OH}^{-}$(Sparks 2003). Both Fe and $\mathrm{Al}$ are highly hydrolysed at neutral $\mathrm{pH}$ and occur as hydrated cations at low $\mathrm{pH}$. The target metals $(\mathrm{Ni}, \mathrm{Zn}, \mathrm{Cu}, \mathrm{Pb} \mathrm{Cd})$ do not form stable hydroxo-complexes even at high $\mathrm{pH}$, and are more likely to combine with non- $\mathrm{OH}^{-}$ ligands if possible (Sparks 2003). Thus, at circum neutral pH Fe and Al form hydroxide precipitates while the remaining target metals do not.

Hard and soft characteristics govern the metal binding affinity for a given ligand. Hard cations such as alkali metals and alkaline earth metals interact via electrostatic, ionic reactions while soft cations react to form covalent bonds. Transition metals form complexes of intermediate strength (Sparks 2003). Table 2-5 shows the classification of Lewis acids and bases.

Table 2-5: Classification of Lewis Acids and Bases

\begin{tabular}{lccccc}
\hline \multicolumn{3}{c}{ Lewis Acids } & \multicolumn{3}{c}{ Lewis Bases } \\
\hline \multicolumn{3}{c}{ Group I metals: Hard Acids } & \multicolumn{3}{c}{ Hard Bases } \\
$\mathrm{H}^{+}$ & $\mathrm{Li}^{+}$ & $\mathrm{Na}^{+}$ & $\mathrm{H}_{2} \mathrm{O}$ & $\mathrm{OH}^{-}$ & $\mathrm{F}^{-}$ \\
$\mathrm{K}^{+}$ & $\mathrm{Be}^{2+}$ & $\mathrm{Mg}^{2+}$ & $\mathrm{PO}_{4}^{3-}$ & $\mathrm{SO}_{4}^{2-}$ & $\mathrm{Cl}^{-}$ \\
$\mathrm{Ca}^{2+}$ & $\mathrm{Al}^{3+}$ & $\mathrm{Fe}^{3+}$ & $\mathrm{CO}_{3}^{2-}$ & $\mathrm{ClO}_{4}^{-}$ & $\mathrm{NO}_{3}^{-}$ \\
Group II metals: Transition Acids & & Transition Bases & \\
$\mathrm{Cr}^{2+}$ & $\mathrm{Mn}^{2+}$ & $\mathrm{Fe}^{2+}$ & $\mathrm{Br}^{-}$ & $\mathrm{NO}_{2}^{-}$ & $\mathrm{N}_{2}$ \\
$\mathrm{Co}^{2+}$ & $\mathrm{Ni}^{2+}$ & $\mathrm{Cu}^{2+}$ & & & \\
$\mathrm{Group} \mathrm{III} \mathrm{metals:} \mathrm{Soft}^{2+} \mathrm{Acids}^{2+}$ & & $\mathrm{Soft} \mathrm{Bases}$ \\
$\mathrm{Ag}^{+}$ & $\mathrm{Au}^{+}$ & $\mathrm{Tl}^{+}$ & $\mathrm{I}^{-}$ & $\mathrm{CN}^{-}$ & $\mathrm{CO}$ \\
$\mathrm{Cu}^{+}$ & $\mathrm{Zn}^{2+}$ & $\mathrm{Cd}^{2+}$ & & & \\
$\mathrm{Hg}^{2+}$ & $\mathrm{Pb}^{2+}$ & $\mathrm{Sn}^{2+}$ & & & \\
\hline
\end{tabular}

(Sparks 2003)

Group I metals prefer hard ligands, but form weak complexes with them. Complexation of group I metals therefore only occurs under high ligand and metal concentrations. Group III metals have a greater affinity for softer sites, and form strong complexes with them at relatively low concentrations (Sparks 2003). 


\section{Literature Review}

Group II metals can react with all three ligand groups, resulting in complex interrelationships. Group II metals compete for hard base binding sites due to formation of stronger complexes than group I metals. Group II metals are unlikely to completely dominate hard base complexation due to relatively low concentration, compared to group I metals (Sparks 2003). Conversely, group II metals occur at a higher concentration than group III metals but form weaker complexes with soft bases, resulting in competition between group II and III metals for soft ligand sites (Sparks 2003).

The formation of metal complexes results in reduced metal bioavailability and toxicity (Forstner, Wittmann et al. 1981), and is discussed in section 2.1.2.1.2. A discussion on chemical equilibrium and speciation may be found in Snoeyink and Jenkins (1980).

\subsubsection{Trace Metal Adsorption/co-precipitation}

Co-precipitation is the simultaneous removal of foreign ions during the formation of primary metal precipitates, such as $\mathrm{Fe}$ and $\mathrm{Al}$ oxides (Lee, Bigham et al. 2002). Trace metals may be incorporated into major precipitates as impurities to the crystal lattice (Lottermoser 2003). Adsorption is the accumulation of an ion at the interface between an inert or precipitated suspended solid and the aqueous phase (Lee, Bigham et al. 2002).

Crawford (1993) showed that co-precipitation and adsorption of Zn occur simultaneously at a $\mathrm{Zn}$ to iron oxide ratio of 1:5, with approximately $80 \%$ of $\mathrm{Zn}$ removed by either process at $\mathrm{pH}$ 7. As co-precipitation and adsorption are difficult to differentiate, they are generally referred to as adsorption from this point on.

At neutral to alkaline $\mathrm{pH}$, recently formed $\mathrm{Fe} / \mathrm{Al}$ oxide precipitates may adsorb dissolved trace metals. The point of zero charge (PZC) of oxide precipitates varies with solution and oxide characteristics. Typically, the surface charge becomes negative at circumneutral to alkaline $\mathrm{pH}$, creating a sorption potential for dissolved cationic metals (Webster 1995). The $\mathrm{PZC}$ of $\mathrm{Al}(\mathrm{OH})_{3}$ occurs at $\mathrm{pH} 5$, while the $\mathrm{PZC}$ of other aluminium and iron oxides range from pH 6.5 to 8.5 (Evangelou 1998; Sparks 2003).

Davies et al. (2011a) positively correlated a decrease in dissolved $\mathrm{Zn}$ with an increase in $\mathrm{Zn}$ associated with Fe-rich precipitates, found in the zone of AMD neutralisation at $\mathrm{pH}$, suggesting $\mathrm{Zn}$ adsorption to Fe-rich precipitates occurred at relatively low $\mathrm{pH}$.

Webster et al. (1998) used batch experiments to show the $\mathrm{pH}$ dependant adsorption of $\mathrm{Pb}$, $\mathrm{Cu}, \mathrm{Zn}$ and $\mathrm{Cd}$ onto natural AMD oxides collected from an AMD impacted stream. At $\mathrm{pH}$ 
7 and a 1:11 ratio of metal to AMD oxide, 70 and $87 \%$ of $\mathrm{Zn}$ and $\mathrm{Cd}$ were removed from $\mathrm{AMD}$, mostly due to adsorption to precipitated metal oxides. Extraction of $\mathrm{Pb}$ and $\mathrm{Cu}$ was also increased by $50 \%$ through AMD precipitate adsorption. These results support Table 2-4 which states hydroxide precipitation of $\mathrm{Pb}$ and $\mathrm{Cu}$ may have partially occurred at $\mathrm{pH}$ 7, but $\mathrm{Zn}$ and $\mathrm{Cd}$ precipitation would not occur until $\mathrm{pH} 8.4$ and 9.7, respectively. A similar trend has been shown for $\mathrm{Ni}$ (at a similar sorbent to metal concentration ratio) where $66 \%$ of $\mathrm{Ni}$ was sorbed to hydrous iron oxide at $\mathrm{pH} 7$ (Xu, Axe et al. 2007). Hydroxide precipitation of $\mathrm{Ni}$ would also be unlikely at $\mathrm{pH} 7$.

Lee et al. (2002) showed that the sorption of trace metals to precipitates formed by AMD neutralisation was dependant on the relative concentrations of $\mathrm{Fe}, \mathrm{Al}$, and $\mathrm{Mn}$ in raw AMD. The AMD samples dominated by $\mathrm{Fe}$ and $\mathrm{Al}$ (which precipitate at $\mathrm{pH}<4$ and 5 , respectively) resulted in between 70 and $100 \%$ adsorption of $\mathrm{Cu}, \mathrm{Zn}, \mathrm{Ni}$, and $\mathrm{Cd}$ at $\mathrm{pH} 7$. For Mn dominated AMD, approximately $25 \%$ of $\mathrm{Ni}, \mathrm{Zn}$, and $\mathrm{Cd}$ were adsorbed at $\mathrm{pH} 7$. As $\mathrm{Mn}$ does not precipitate (significantly) below $\mathrm{pH} 8$ fewer oxide reaction sites were available at $\mathrm{pH} 7$, resulting in less sorption. Sorption of $\mathrm{Pb}$ was high for all AMD types, due to a high affinity for $\mathrm{Pb}$ binding to precipitated iron oxide, even in low Fe AMD. The data showed sorption of $\mathrm{Cu}$ to iron and aluminium oxides was also significant although partial hydroxide precipitation of $\mathrm{Cu}$ may have occurred.

Due to the slow dissociation of $\mathrm{CaCO}_{3}$ at circum-neutral $\mathrm{pH}$, significant residual calcite accumulates in sludge after AMD neutralisation. The PZC of calcite varies, but typically occurs over pH 8 (Wolthers, Charlet et al. 2008). The adsorption of trace metals to calcite provides an additional metal removal mechanism (García-Sánchez and Álvarez-Ayuso 2002) for the $\mathrm{CaCO}_{3}$ neutralised scenarios. Several researchers have quantified the adsorption capacity of calcite for metals. The adsorption capacity of calcite for $\mathrm{Zn}$ and $\mathrm{Cd}$ is 185 and $16.3 \mathrm{mg} / \mathrm{g}$, respectively (García-Sánchez and Álvarez-Ayuso 2002). Lakshtanov and Stipp (Lakshtanov and Stipp 2007) showed that Ni sorption to calcite was low with approximately $8-10 \%$ of $\mathrm{Ni}$ adsorbed to calcite over a range of $\mathrm{Ni}$ and calcite concentrations. The adsorption capacity of calcite for $\mathrm{Pb}$ was also relatively low at around $0.26 \mathrm{mg} / \mathrm{g}$ (Rouff, Elzinga et al. 2005).

\subsubsection{Alkalinity Reagents}

A range of alkalinity reagents are used for neutralisation of AMD. Table 2-6 lists the three alkalinity reagents used in this thesis. 
Table 2-6: Alkalinity reagents used in AMD neutralisation

\begin{tabular}{cccc}
\hline Common Name & Chemical Name & $\begin{array}{c}\text { Neutralization } \\
\text { Efficiency }^{*}\end{array}$ & $\begin{array}{c}\mathbf{2 0 0 0} \text { Bulk Cost } \\
(\mathbf{\$} / \mathbf{T})\end{array}$ \\
\hline Limestone & $\mathrm{CaCO}_{3}$ & $30-70 \%$ & $143^{* *}$ \\
Hydrated Lime & $\mathrm{Ca}(\mathrm{OH})_{2}$ & $90 \%$ & $415^{* * *}$ \\
Caustic Soda (solid) & $\mathrm{NaOH}$ & $100 \%$ & $1100^{* * *}$ \\
Caustic Soda (50\% soln.) & $\mathrm{NaOH}$ & $100 \%$ & $575^{* * *}$ \\
\hline
\end{tabular}

* Based on required alkalinity dose to neutralise acidity

** (Weber 2011)

**** (Fraser 2011)

adapted from (Skousen, Sexton et al. 2000)

Other alkalinity sources may be used in more specialised treatment of AMD including calcium oxide, sodium carbonate and anhydrous ammonia (Skousen, Sexton et al. 2000). Process by-products including cement kiln dust, coal fly ash and steel making slags are also a source of alkalinity (Skousen, Sexton et al. 2000). The selection of a specific alkalinity reagent is based on a range of variables, shown in Table 2-7.

Table 2-7: Factors affecting selection of alkalinity reagent for AMD neutralisation

\begin{tabular}{cc}
\hline Technical & Other \\
\hline Acid loads & Reagent cost \\
Flow rate & Labour \\
Types and concentration of metals & Machinery/equipment/plant \\
Neutralisation efficiency & Treatment lifetime \\
Maximum pH attainable & Health and safety \\
Sludge settling rates/volume/density & Security of supply
\end{tabular}

(Skousen, Sexton et al. 2000; Trumm 2010)

For example, $\mathrm{CaCO}_{3}$ is a relatively cheap chemical reagent but slow dissolution kinetics limit its application to long residence time scenarios, which allow greater dissolution (Skousen, Sexton et al. 2000). Alternatively, $\mathrm{NaOH}$ is a relatively expensive source of alkalinity and has the greatest maximum $\mathrm{pH}$ attainable of common alkalinity sources. However, its application is limited, partly because neutralisation by $\mathrm{NaOH}$ is dangerously exothermic from a health and safety perspective (Trumm 2010). 


\subsubsection{Treatment Processes}

AMD treatment processes are site specific due to variations in the composition of AMD discussed in section 2.1.1. Acid mine drainage treatment processes are generally designed to:

- Raise $\mathrm{pH}$ and remove acidity;

- Reduce volume;

- Lower dissolved metal and sulphate concentrations;

- Lower the bioavailability of metals in solution;

- Oxidize or reduce the solution; or

- Collect, dispose or isolate the hazardous fraction.

(Lottermoser 2003)

Treatment of AMD may be achieved by active and passive systems. Trumm (2010) discusses the factors which influence the selection of either an active or passive AMD treatment system.

\subsubsection{Active Treatment}

Active treatment systems require continuous inputs of both chemicals and energy. Inputs include solid/liquid/gas phase chemical reagents, active maintenance, monitoring, and mechanical devices to drive treatment processes (Younger, Banwart et al. 2002). Active treatment often requires sludge management and disposal, adding a significant cost to the approach (Skousen, Sexton et al. 2000). Active treatment is typically used on operational mine sites due to land area restrictions, variable AMD chemistry and flows, and availability of resources (Trumm 2010).

\subsection{Neutralisation (DAOS)}

The predominant active AMD treatment method is neutralisation by the DAOS process; Dosing with Alkalinity, Oxidation and Sedimentation (Trumm 2010). Pre-treatment may be required for specific AMD neutralisation. This may include a pre-neutralisation sedimentation step for AMD with significant suspended solid (SS) loads or a degassing step for highly carbonated waters to reduce carbonate buffering (Younger, Banwart et al. 2002).

The aim of alkalinity dosing is to raise the $\mathrm{pH}$ to circum-neutral levels to neutralise acidity and trigger hydroxide precipitation of metals, as discussed in section 2.2.1. Dosing methods depend on the reactivity of the alkaline reagent. Low solubility neutralisation 
chemicals $\left(\mathrm{Ca}(\mathrm{OH})_{2}\right.$ and $\left.\mathrm{CaCO}_{3}\right)$ are dispensed as a slurry to AMD during an initial rapid mixing phase, while soluble reagents $(\mathrm{NaOH})$ may be metered directly into the AMD (Younger, Banwart et al. 2002). The alkalinity dose rate is usually controlled by a downstream $\mathrm{pH}$ probe to maintain a constant effluent $\mathrm{pH}$.

The primary objective of the oxidation phase is to ensure sufficient dissolved oxygen is available to oxidise metals ( $\mathrm{Fe}$ and $\mathrm{Mn}$ ) to their least soluble form through reactions similar to Equation 2-2, enabling precipitation at the lowest pH possible (Skousen, Sexton et al. 2000). Oxidation is achieved through physical, chemical and biological processes which are discussed in Younger et al. (2002).

The oxidation step may not be required if metals are already highly oxidised after the alkalinity dosing step (Trumm 2010). The AMD impacted streams on the West Coast of the South Island have a high dissolved oxygen concentration (Greig, Niyogi et al. 2010), resulting from turbulence induced oxygen entrainment. Thus, the oxidation step may not be required for treatment of West Coast mine drainages.

The aim of the sedimentation step is to remove SS, which may be derived from suspended sediments or precipitated metal hydroxides (Younger, Banwart et al. 2002). Precipitated particles may naturally coalesce, forming a loose, open-structured floc which slowly settles due to a density slightly greater than water (Skousen, Sexton et al. 2000; Younger, Banwart et al. 2002; Tchobanoglous, Burton et al. 2003). Due to the high concentration of $\mathrm{Al}$ and $\mathrm{Fe}$ in raw AMD, sufficient metal hydroxides are produced during neutralisation to trigger zone 4 settling, shown on Figure 2-1.

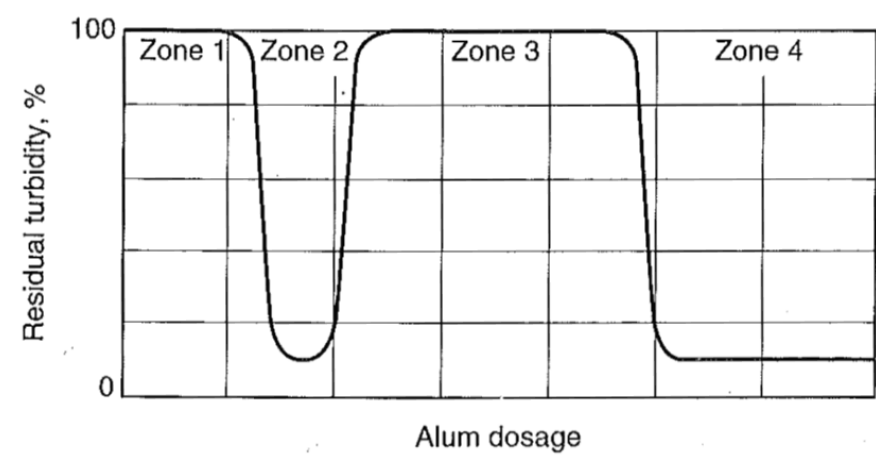

\section{Figure 2-1: Destabilisation and flocculation of colloidal particles}

Zone 4 settling is characterised as sweep floc settling (Tchobanoglous, Burton et al. 2003) where during the settling phase the water column is separated into the sludge settling zone 
and clear supernatant by the interface. Thus, the supernatant turbidity and SS of neutralised AMD is low, provided alkalinity reagent dissolution efficiency is high, which is not the case for $\mathrm{CaCO}_{3}$.

Figure 2-1 shows how negatively charged particles are removed from suspension by addition of Alum, or positively charged aluminium cations. Negatively charged SS repel each other, remaining in suspension in zone 1. The surface of SS particles is then neutralised by aluminium cations in zone 2 , resulting in particle agglomeration and decreased turbidity. When the system is overdosed with aluminium cations, (as in zone 3) the particles become positively charged and repel each other, resulting in an increased turbidity.

Sedimentation may be achieved using ponds which provide sufficient residence time for a high degree of particle settlement. Younger et al. (2002) states that required pond residence time may exceed 24 hours for unassisted floc sedimentation. Thus, in applications with strong AMD and/or high flow rates, available land area may prevent the use of sedimentation ponds (Trumm 2010).

Where sedimentation ponds are infeasible, specifically designed plant such as clarifiers may be required (Trumm 2010). Clarification processes are enhanced by a preceding coagulation and flocculation step. Addition of coagulants reduces electrostatic repulsive charges between individual particle surfaces, allowing small particles to join together and form flocs (Younger, Banwart et al. 2002). Flocculation may be enhanced by addition of polymers which facilitate particle bridging. Polymers bridge two or more particles along one polymer strand. Accumulation of polymer strands rapidly increases the particle size of floc, resulting in increased settling kinetics (Skousen, Sexton et al. 2000; Tchobanoglous, Burton et al. 2003). The process of flocculation involves gentle mixing to encourage floc contact and aggregation (Tchobanoglous, Burton et al. 2003).

Final dewatering is commonly achieved by plate and frame pressure filtration, belt pressure filtration and vacuum filtration; or less commonly centrifugal force (Trumm 2010).

\subsection{Chemical Treatments}

Chemical treatments do not rely on neutralisation induced precipitation. They rely on oxidation, reduction, precipitation, adsorption or cation exchange processes (Lottermoser 
Literature Review

2003). Chemical treatments are used to target specific contaminants that require removal through a specialised process. For example, the addition of oxidants converts dissolved $\mathrm{Fe}^{2+} / \mathrm{Mn}^{2+}$ to insoluble $\mathrm{Fe}^{3+} / \mathrm{Mn}^{3+}$ and convert toxic $\mathrm{As}^{3+}$ to less toxic $\mathrm{As}^{5+}$ (Lottermoser 2003). Other chemical treatment methods include sulphidisation, sorption and ion exchange and membrane processes. Younger et al. (2002) describes these and other specialist chemical treatment processes. 


\subsubsection{Passive Treatment}

Passive treatment methods use chemical and biological processes to convert reactive dissolved metals and acidity into non-reactive forms in the substrate (O'Sullivan 2005). Passive treatment systems operate under minimal external energy and chemical inputs and are most suited to closed mine sites requiring long term treatment (Trumm 2010). This section discusses passive systems whose primary treatment component is neutralisation. Other passive treatment systems for AMD (O'Sullivan 2005; Trumm 2010) are not discussed here.

Oxidation or reduction processes are used to remove metals from AMD in passive treatment systems. Oxidation processes drive the pyrite oxidation reaction to completion, resulting in hydroxide precipitation. Reduction processes reverse the pyrite oxidation reaction, by creating a reducing environment, resulting in precipitation of iron and hydrogen sulphides (Trumm, Watts et al. 2006).

\subsection{Oxidising Strategies}

Passive treatment technologies which utilise oxidation include open limestone channels (OLC), diversion wells and limestone leaching beds (Trumm 2010). Open limestone channels consist of an impermeable ditch (open to the atmosphere) lined with high quality, crushed $\mathrm{CaCO}_{3}$ (O'Sullivan 2005). Alkalinity released from the OLC on contact with AMD neutralises acidity by Equation 2-15.

$$
\begin{gathered}
\mathrm{CaCO}_{3(s)}+\mathrm{H}_{(a q)}^{+} \leftrightarrow \mathrm{Ca}_{(a q)}^{2+}+\mathrm{HCO}_{3}^{-} \\
\mathrm{CaCO}_{3} \text { acidity neutralisation }
\end{gathered}
$$$$
\text { Equation 2-15 }
$$

(Watzlaf, Schroder et al. 2004)

Neutralisation of acidity causes precipitation of $\mathrm{Al}$ and Fe hydroxides. Open limestone channels perform best on slopes greater than $20 \%$, which create sufficient turbidity to ensure oxygenation and maintain suspension of precipitated metals, thus preventing $\mathrm{CaCO}_{3}$ armouring (Skousen, Sexton et al. 2000). Diversion wells and limestone leaching beds operate on a similar principle, where AMD is channelled through a $\mathrm{CaCO}_{3}$ filled structure to promote neutralisation.

\subsection{Reducing Strategies}

The anoxic limestone drain (ALD) is the simplest example of a passive, reducing method for treatment of AMD. Anoxic limestone drains consist of a shallow trench backfilled 


\section{Literature Review}

with crushed $\mathrm{CaCO}_{3}$, which is then sealed using plastic or other impermeable material to prevent oxygen transfer (O'Sullivan 2005). Again, the $\mathrm{CaCO}_{3}$ acts as a source of alkalinity, neutralising acidity and triggering precipitation of metals. Provided influent AMD has a low dissolved oxygen concentration, hydrolysis of dissolved metals within the ALD does not occur, preventing armouring of $\mathrm{CaCO}_{3}$ (Trumm 2010).

Typically ALD use is restricted to AMD with $\mathrm{Fe}^{3+}$ and dissolved oxygen concentrations of less than $1 \mathrm{mg} / \mathrm{L}$ and $\mathrm{pH}>5$, to prevent armouring of $\mathrm{CaCO}_{3}$ (Lottermoser 2003; Watzlaf, Schroder et al. 2004). The ALD target effluent $\mathrm{pH}$ is between 6 and 7. Controlled aeration of the ALD effluent in downstream ponds causes rapid oxidation of dissolved metals, triggering hydrolysis and precipitation of metal hydroxides (Lottermoser 2003).

Individual passive treatment systems are unreliable as a single permanent solution for AMD management (Skousen, Sexton et al. 2000). Reducing alkalinity producing systems (RAPS) use a series of treatment phases to optimise passive AMD treatment. A simple RAPS involves a submerged organic compost layer overlaying a crushed $\mathrm{CaCO}_{3}$ bed. The anaerobic compost layer strips oxygen and reduces Fe and sulphate, preventing armouring of the underlying alkalinity releasing limestone layer, which neutralised acidity (Skousen, Sexton et al. 2000). 


\subsubsection{ANZECC Guidelines}

The ANZECC water quality guidelines aim to protect and manage the environmental values supported by water resources (ANZECC 2000). These guidelines are not mandatory standards, but may be used by regulators who set metal discharge limits. The ANZECC guidelines are included here as a basis for later metal removal discussion. Table 2-8 shows AZNECC guideline trigger values for protection of $80 \%$ of species, corresponding to a significantly degraded stream (ANZECC 2000).

Table 2-8: AZNECC guideline trigger values for target metals

\begin{tabular}{|c|c|}
\hline Metal & ANZECC $80 \%$ trigger value $(\mathrm{mg} / \mathrm{L})^{*}$ \\
\hline $\mathbf{N i}$ & 1.46 \\
\hline $\mathbf{Z n}$ & 0.61 \\
\hline $\mathrm{Cu}$ & 0.04 \\
\hline Cd & 0.02 \\
\hline $\mathbf{P b}$ & 0.19 \\
\hline
\end{tabular}

${ }^{*}$ For $1000 \mathrm{mg} / \mathrm{L} \mathrm{CaCO}$ Hardness

(ANZECC 2000)

Trigger values vary with hardness due to the detoxification effects of hardness discussed in section 2.1.2.1.2. The hardness of $\mathrm{Ca}(\mathrm{OH})_{2}$ neutralised $\mathrm{AMD}$ was approximately 1000 $\mathrm{mg} / \mathrm{L} \mathrm{CaCO}_{3}$, due primarily to the $400 \mathrm{mg} / \mathrm{L} \mathrm{Ca}$ concentration. The ANZECC guidelines classify $400 \mathrm{mg} / \mathrm{L} \mathrm{CaCO}_{3}$ as extremely hard, so the hardness algorithm may be unreliable when extrapolated to $1000 \mathrm{mg} / \mathrm{L} \mathrm{CaCO}_{3}$ (although no suggestion of this was made in the document). Therefore these trigger value guidelines are used for comparison only and are not set as neutralisation metal removal targets. The ANZECC guidelines also suggest a range of trigger values for turbidity in upland rivers of between 2 and 25 NTU. 


\subsubsection{AMD Prevention}

During mine operation, exposure of work face material and waste rock piles is inevitable and minimal AMD prevention is possible. After mine closure and rehabilitation, seeps, tailings, and exposed rock act as AMD sources, producing relatively small quantities of AMD. Post-mining AMD control is achieved by preventing exposure of waste pyritic material to oxygen and water. Impermeable covers are constructed over waste rock heaps, using materials with sufficiently low permeability to prevent water and oxygen ingress. Wet, dry and sponge covers are used where appropriate as discussed by Lottermoser (2003).

Isolation techniques minimise acid formation by co-disposal and encapsulation of PAF waste rock in inert material (Lottermoser 2003). Incorporation of alkaline material, instead of benign waste in the isolation techniques could result in a no net acidity production from waste rock heaps. The ratio of alkaline material to waste rock is typically determined by acid base accounting (ABA) (Skousen, Sexton et al. 2000).

Adits, shafts and tunnels are common point sources of AMD from underground mines. Closing of adits using concrete or grout hydraulic seals minimises groundwater escape from underground workings (Lottermoser 2003). If the seal is air and water tight, workings below the water-table will eventually flood preventing atmospheric oxygen entering the mine and limiting pyrite oxidation. Flooding can result in reactivation of springs under restored hydrostatic pressure, resulting in AMD seeps (Lottermoser 2003). Catastrophic failure of the adit seals can result in the release of significant AMD reservoirs (Somerfield, Michael Gee et al. 1994). Other methods of post treatment AMD control include alkaline recharge trenches and bactericides (Skousen, Sexton et al. 2000). 


\section{Literature Review}

\subsection{HS for AMD Treatment}

This section discusses the potential for HS incorporation into the AMD neutralisation process. The first section describes the properties of HS which are fundamental to HSmetal complexation. The second section describes the process of HS-metal complexation and identifies HS-metal complexation capacities cited from the literature. The third section briefly discusses the limited publications regarding specifically lignite derived HS application to metal contaminated waters. The final section discusses process considerations for HS application to AMD neutralisation.

\subsubsection{Humic Substance Properties}

Schnitzer and Kahn (1972) characterised humic substances (HS) as "amorphous, brown or black, hydrophilic, acidic, poly-disperse substances with molecular weights ranging from several hundreds to tens of thousands". Thus, HS are complex in nature and have a range of interactions with the natural environment and metals within it.

Humic substances are found in soils, natural waters, sewage, compost, marine and lake sediments, peat bogs, carbonaceous shale, lignite, brown coals and other deposits (Stevenson 1994). The concentration of HS in West Coast streams range from $0-30$ mg/L (Collier and Winterbourn 1987; Collier 1989). 
The molecular structure of HS is highly variable. Figure 2-2 shows a theoretical HA structure derived from analysis of soil extracted HS (Schulten and Schnitzer 1993).

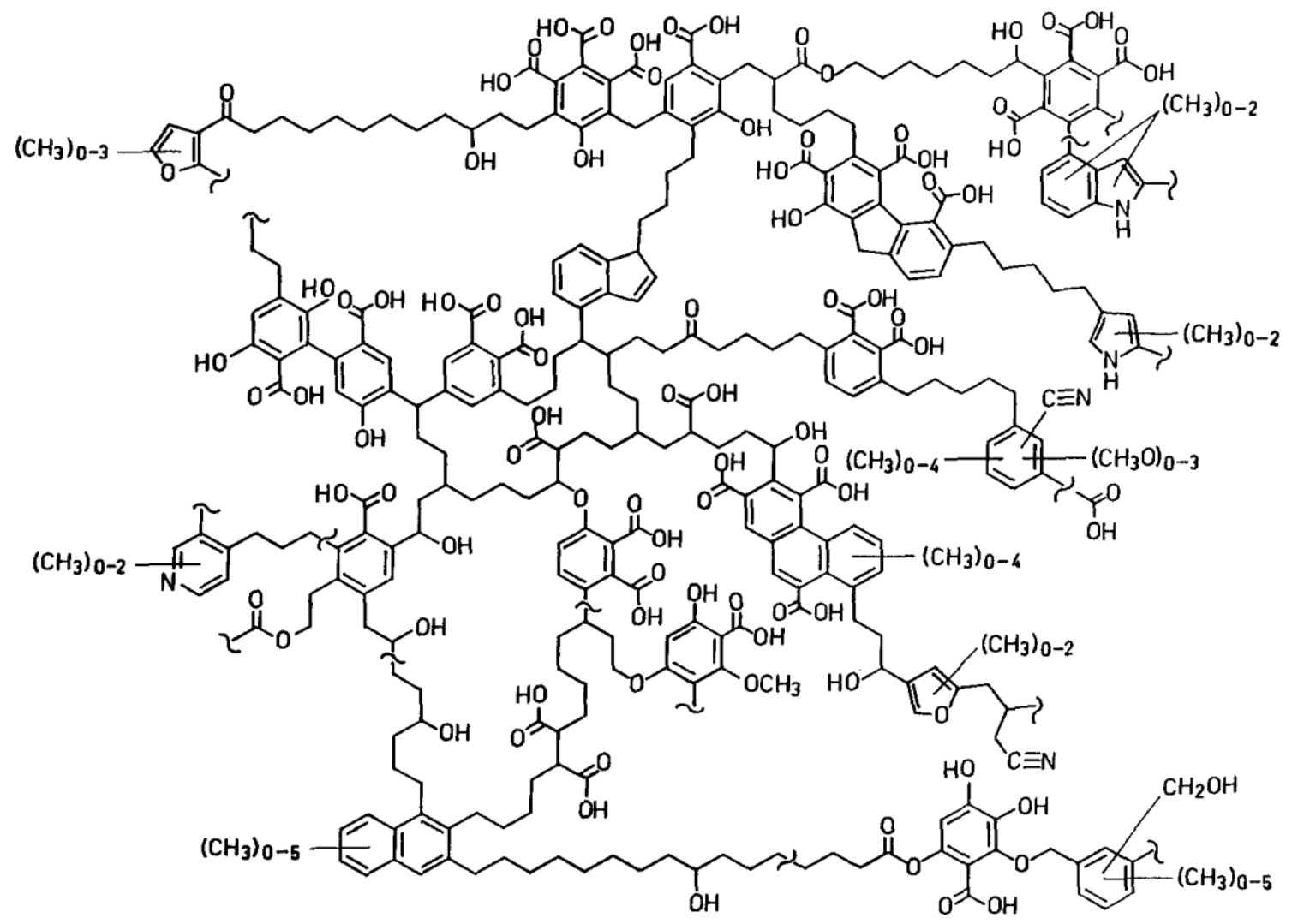

Figure 2-2: Theoretical HA structure

(Schulten and Schnitzer 1993)

Due to their structural variability, HS are classified by isolation procedure, rather than specific molecular features (Schnitzer and Kahn 1972). The most common HS classification is made based on alkali and acid solubility, where HS are divided into three groups of compounds; fulvic acid, humic acid and humin, as shown in Table 2-9.

Table 2-9: HS classification based on acid/alkali solubility

\begin{tabular}{ccc}
\hline & Alkali & Acid \\
$(\mathrm{pH} \sim 10-11)$ & $(\mathrm{pH}<1)$ \\
\hline Fulvic Acid & Soluble & Soluble \\
Humic Acid & Soluble & Insoluble \\
Humin & Insoluble & Insoluble
\end{tabular}

(Schnitzer and Kahn 1972) 


\section{Literature Review}

The International Humic Substance Society (IHSS) uses the acid/alkali solubility characteristics of HS in the extraction of HA and FA in their accepted method (Swift 1996). Schnitzer and Kahn (1972) and Stevenson (1994) broadly characterised HA and FA elemental composition, molecular weight and functional groups, as shown in Table $2-10$.

Table 2-10: Physical and chemical properties of humic and fulvic acids.

\begin{tabular}{lccc}
\hline \multicolumn{1}{c}{ Property } & & Humic Acids & Fulvic Acids \\
\hline Elemental Composition & $\mathrm{C}$ & $50-60$ & $40-50$ \\
$(\mathrm{wt} \%)$ & $\mathrm{H}$ & $3-6$ & $4-7$ \\
& $\mathrm{O}$ & $30-38$ & $40-50$ \\
& $\mathrm{~N}$ & $0.8-4.3$ & $0.9-3.3$ \\
& $\mathrm{~S}$ & $0.1-2$ & $0.1-3.6$ \\
Molecular weight & average & $50,000-100,000$ & $500-2,000$ \\
$(\mathrm{~g} /$ mol) & Carboxyl & $14-50$ & $58-75$ \\
Functional Group & Phenol & $7-38$ & $1-19$ \\
$(\%$ of oxygen in group) & Alcohol & $13-15$ & $11-35$ \\
& Carbonyl & $4-30$ & $4-14$ \\
\hline
\end{tabular}

(Schnitzer and Kahn 1972; Stevenson 1994)

The properties of HA and FA have not been significantly revised since the early work of Schnitzer and Kahn; (Monteil-Rivera, Brouwer et al. 2000; Novak, Kozler et al. 2001; Kurková, Klika et al. 2004; Havelcová, Mizera et al. 2009). However, the properties of HS do vary depending on parent material and extraction method (Chen, Gu et al. 2002; Kurková, Klika et al. 2004; Weber, Tang et al. 2006).

Humic acids are larger than fulvic acids, with a greater aromacity and therefore carbon concentration. Humic acids have a lower oxygen content and thus a lower functional group density, while fulvic acids are more aliphatic, resulting in a greater functional group density and higher oxygen content. The major functional groups present on both HA and FA are shown in Table 2-11. 
Literature Review

Table 2-11: Important HS functional groups

\begin{tabular}{cc}
\hline \multicolumn{2}{c}{ Functional Group } \\
Acidic Groups \\
Carboxyl & $\mathrm{R}-\mathrm{C}=\mathrm{O}(-\mathrm{OH})$ \\
Phenolic $\mathrm{OH}$ & $\mathrm{Ar}-\mathrm{OH}$ \\
Alcohol & $\mathrm{R}-\mathrm{CH}=\mathrm{CH}-\mathrm{OH}$ \\
Carbonyl & $\mathrm{Ar}=\mathrm{O}$ \\
Neutral Groups & $\mathrm{R}-\mathrm{CH}-\mathrm{OH}$ \\
Alcoholic $\mathrm{OH}$ & $\mathrm{R}-\mathrm{CH}{ }_{2}-\mathrm{O}-\mathrm{CH}{ }_{2}-\mathrm{R}$ \\
Ether & $\mathrm{R}-\mathrm{C}=\mathrm{O}(-\mathrm{R})$ \\
Ketone & $\mathrm{R}-\mathrm{C}=\mathrm{O}(-\mathrm{H})$ \\
Aldehyde & $\mathrm{R}-\mathrm{C}=\mathrm{O}(-\mathrm{OR})$ \\
Ester & R-C $=\mathrm{O}(-\mathrm{NH}-\mathrm{R})$ \\
\hline Amine & Basic Groups \\
&
\end{tabular}

(Sparks 2003)

Metal affinities for ligand binding vary according to the ligand type. The most important chelating groups, in order of decreasing affinity for metal ions are:

$$
\begin{aligned}
& -\mathrm{O}^{-} \\
& \text {enolate }
\end{aligned}>\begin{aligned}
& -\mathrm{NH}_{2} \\
& \text { amine }
\end{aligned}>\begin{aligned}
& -\mathrm{N}=\mathrm{N}- \\
& \text { azo }
\end{aligned}>\begin{aligned}
& =\mathrm{N}- \\
& \text { ring } \mathrm{N}
\end{aligned}>\begin{aligned}
& -\mathrm{COO}- \\
& \text { carboxylate }
\end{aligned}>\begin{aligned}
& -\mathrm{O}- \\
& \text { ether }
\end{aligned}>\begin{aligned}
& -\mathrm{C}=\mathrm{O} \\
& \text { carbonyl }
\end{aligned}
$$

(Schnitzer and Kahn 1972; Sparks 2003)

Typically nitrogen containing functional groups are more likely to form complexes with metals, however, nitrogen containing functional groups on HS are relatively rare (Schnitzer and Kahn 1972). Sulphur containing groups may also be responsible for significant binding of polarisable metals, such as mercury (Tipping 2002). Overall, the relatively high concentration and low binding affinity oxygen containing functional groups (carboxylic and phenolic) dominate humic-metal complexation. Typically only 


\section{Literature Review}

carboxylic and phenolic reactive groups are considered in HS-metal complexation modelling (Christl, Milne et al. 2001; Milne, Kinniburgh et al. 2003).

The dissociation constants for the acid groups were determined by Milne et al. (2001) and are shown on Table 2-12.

Table 2-12: Dissociation constants of HA and FA functional groups $\left(\log K_{i}\right)$

\begin{tabular}{ccc}
\hline & Humic Acid & Fulvic Acid \\
\hline Carboxylic & 2.93 & 2.34 \\
Phenolic & 8.0 & 8.6 \\
\hline
\end{tabular}

(Milne, Kinniburgh et al. 2001)

These dissociation constants indicate the $\mathrm{pH}$ at which carboxylic and phenolic groups become deprotonated. Deprotonation of these groups allows greater metal complexation due to reduced binding competition from hydrogen ions. The protonation/deprotonation status of the functional groups also affects the overall charge of the HS molecule; HS exhibit a stronger negative charge at higher $\mathrm{pH}$ due to a greater degree of deprotonation (Tipping 2002). 
Literature Review

\subsubsection{HS-Metal Complexation}

Ligands available for metal complexation in natural freshwaters include:

1. Simple inorganic ligands - major anions whose donor atom is oxygen; $\mathrm{CO}_{3}{ }^{2-}$, $\mathrm{SO}_{4}{ }^{2-},=\mathrm{S}-\mathrm{OH}$

2. Hard sites on humic acid - mainly carboxyl and phenolic sites; $-\mathrm{COOH},-\mathrm{OH}$

3. Soft sites on humic acid $-\mathrm{N}$ and $\mathrm{S}$ containing sites; $\mathrm{N}_{\text {org }}, \mathrm{S}_{\text {org }}$.

(Sparks 2003)

Metal complexation by simple inorganic ligands was discussed section 2.2.1.2. Addition of HS to natural waters introduces primarily carboxylic and phenolic ligands capable of metal complexation.

\subsubsection{Stability Constants}

Milne et al. (2003) compiled 171 datasets of literature and experimental data for metal/ion binding by HS and determined generic stability constants for metal complexation at carboxylic and phenolic sites on HA and FA. The derived stability constants are shown in Table 2-13.

Table 2-13: Stability constants for HS complexation by functional group

\begin{tabular}{ccccc}
\hline & \multicolumn{2}{c}{ Humic Acid } & \multicolumn{2}{c}{ Fulvic Acid } \\
& Carboxylic & Phenolic & Carboxylic & Phenolic \\
\hline $\mathbf{F e}^{3+}$ & 3.5 & 17.5 & 6.0 & 36 \\
$\mathbf{A l}^{3+}$ & -1.05 & 8.89 & -4.11 & 12.16 \\
$\mathbf{P b}^{2+}$ & 1.25 & 4.84 & -1.16 & 6.92 \\
$\mathbf{C u}^{2+}$ & 2.23 & 6.85 & 0.26 & 8.26 \\
$\mathbf{Z n}^{2+}$ & 0.11 & 2.39 & -3.84 & -0.73 \\
$\mathbf{N i}^{2+}$ & -0.26 & 1.0 & -2.07 & 2.03 \\
$\mathbf{C d}^{2+}$ & -0.2 & 2.37 & -0.97 & 0.50
\end{tabular}

(Milne, Kinniburgh et al. 2003)

The stability constant for carboxylic and phenolic metal complexes varies, suggesting some metals form HS complexes more readily than others. Rearranging Equation 2-14 yields: 
Literature Review

$$
K_{i} \quad[M]\left[L_{i}\right]=\left[M L_{i}\right]
$$

Equation 2-16

Conditional stability constant

Where, $\mathrm{K}_{\mathrm{i}}$ is the stability constant

$\mathrm{M}$ is the dissolved metal concentration $(\mathrm{mol} / \mathrm{L})$

$\mathrm{L}_{\mathrm{i}}$ is the dissolved ligand concentration $(\mathrm{mol} / \mathrm{L})$

$\mathrm{ML}_{\mathrm{i}}$ is the metal complex concentration $(\mathrm{mol} / \mathrm{L})$.

A $\log \mathrm{K}$ value greater than one suggests that HS complexed would be the dominant metal speciation, assuming sufficient ligand is available. Milne et al. (2003) showed phenolic groups had $\log \mathrm{K}$ values greater than one for all metals on HA and FA (except $\mathrm{Zn}$ ); suggesting phenolic groups would form complexes with most metals. Carboxylic groups were less effective at forming metal complexes with $\mathrm{Cu}$ binding to $\mathrm{FA}$ and $\mathrm{HA}$ while $\mathrm{Pb}$ and $\mathrm{Zn}$ only formed complexes on HA. From these stability constants, a complexation hierarchy was derived for each HS functional group, as shown in Table 2-14.

\section{Table 2-14: Complexation hierarchy of functional groups}

\begin{tabular}{cc}
\hline Group & Complexation hierarchy \\
\hline HA carboxylic & $\mathrm{Cu}>\mathrm{Fe}>\mathrm{Pb}>\mathrm{Zn}>\mathrm{Cd}>\mathrm{Ni}>\mathrm{Al}$ \\
HA phenolic & $\mathrm{Fe}>\mathrm{Al}>\mathrm{Cu}>\mathrm{Pb}>\mathrm{Zn}>\mathrm{Cd}>\mathrm{Ni}$ \\
FA carboxylic & $\mathrm{Fe}>\mathrm{Cu}>\mathrm{Cd}>\mathrm{Pb}>\mathrm{Ni}>\mathrm{Zn}>\mathrm{Al}$ \\
FA phenolic & $\mathrm{Fe}>\mathrm{Al}>\mathrm{Cu}>\mathrm{Pb}>\mathrm{Ni}>\mathrm{Cd}>\mathrm{Zn}$
\end{tabular}

(Milne, Kinniburgh et al. 2003)

Ideally, stability constants would be used to determine the extent of metal complexation at equilibrium for a given metal and ligand concentration. However, equilibrium speciation of a metal is a function of $\mathrm{pH}$, ionic strength, temperature, and binding competition from other dissolved metals; therefore an empirically derived stability constant may only be applied under similar environmental conditions (Snoeyink and Jenkins 1980; Sparks 2003). For example, the Ca concentration of AMD neutralised by $\mathrm{Ca}(\mathrm{OH})_{2}$ is significant. The complexation capacity of HS for alkaline earth metals $(\mathrm{Ca}$, $\mathrm{Mg}$ ) is substantially higher than for alkali ions (Na, K) (Stevenson 1994). Most published data used $\mathrm{NaNO}_{3}$ as background ions. Thus, in an AMD treatment scenario with neutralisation by $\mathrm{Ca}(\mathrm{OH})_{2}$ or $\mathrm{CaCO}_{3}$, competition from $\mathrm{Ca}$ ions for binding sites would result in lower complexation of target metals than predicted by Milne et al. (2003). 


\subsubsection{HS Complexation Mechanisms}

Milne et al. (2003) used the NICA Donnan model to determine the pH dependant metal complexation capacity of a generic HA, shown in Figure 2-3. The model was run using $10 \mathrm{nM}$ free metal ion and $1 \mathrm{mM}$ Ca concentrations.

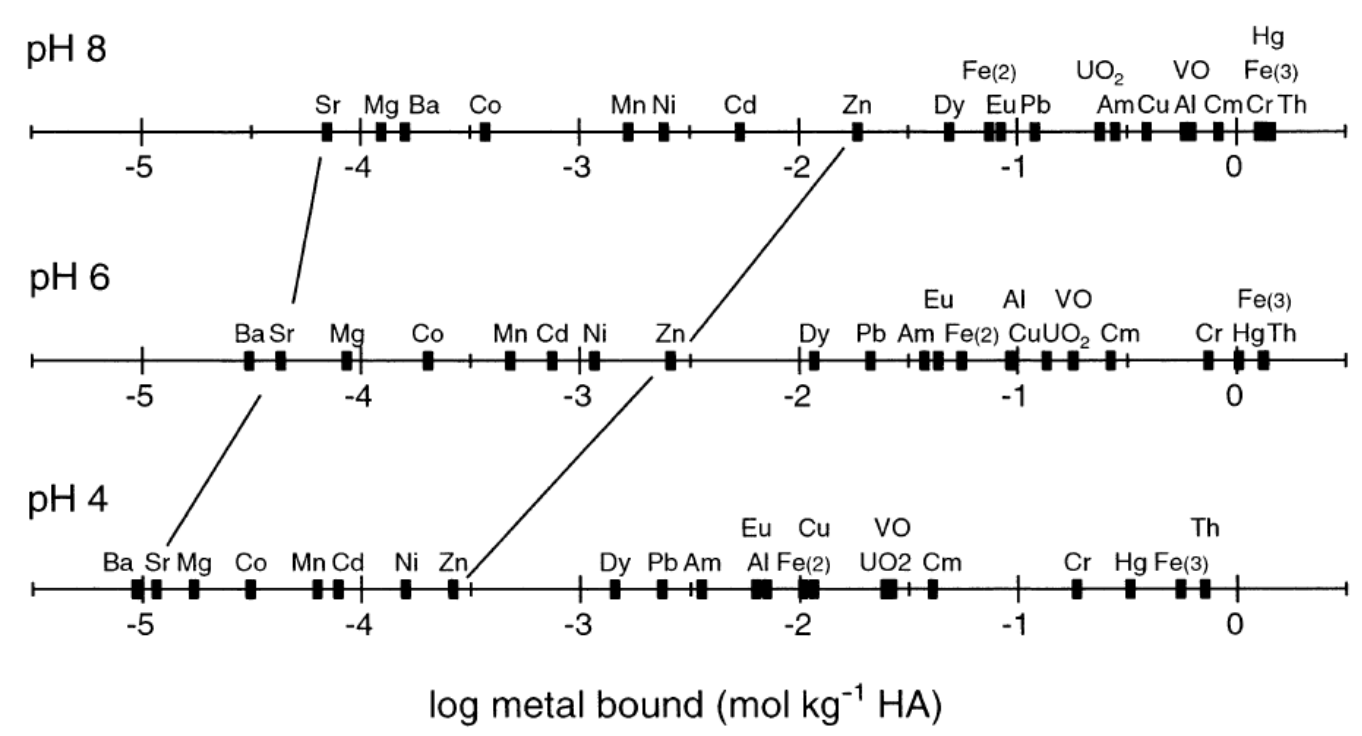

Figure 2-3: pH dependant HA-metal complexation affinity

(Milne, Kinniburgh et al. 2003)

Figure 2-3 shows the complexation affinity of $\mathrm{Cu}, \mathrm{Pb}, \mathrm{Zn}, \mathrm{Ni}$, and $\mathrm{Cd}$ at circum-neutral $\mathrm{pH}$ (i.e. $\mathrm{pH} 8$ ), with up to $25,26,1.3,0.13$ and $0.6 \mathrm{mg} / \mathrm{g}$ bound to HA, respectively.

Figure 2-3 shows the degree of direct complexation of the target metals by HS increases with $\mathrm{pH}$, to a maximum at $\mathrm{pH}$ 8. Dissociation of carboxylic groups occurs in acidic conditions, between pH 3 and 4 (Milne, Kinniburgh et al. 2001; Jochová, Punčochář et al. 2004; Havelcová, Mizera et al. 2009). Above the dissociation pH complexation occurs more easily due to decreased binding competition from hydrogen ions. Phenolic functional groups dissociate between $\mathrm{pH} 8$ and 10 (Milne, Kinniburgh et al. 2001; Havelcová, Mizera et al. 2009), so metal binding to phenolic groups would occur by hydrogen ion exchange.

The complexation capacity of HS has been investigated over a range of different aquatic environments. In non-AMD impacted reservoirs over $70 \%$ of $\mathrm{Cu}, \mathrm{Zn}, \mathrm{Pb}$ and $\mathrm{Cd}$ may be HS complexed, as shown by Linnik and Vasilchuk (2005). Herrmann and Baumgartner (1992) investigated aluminium speciation at the confluence of two AMD impacted streams and rivers. They showed that downstream of the confluence the dominant $\mathrm{Al}$ 
speciation was HS complexed irrespective of the dominant anion. Organically complexed $\mathrm{Al}$ accounted for between 24 to $91 \%$ of the total $\mathrm{Al}$ concentration, suggesting $\mathrm{Al}$ had a greater affinity for DOM than $\mathrm{SO}_{4}{ }^{2-}$ anions, the dominant inorganic ligand in solution. Collier et al. (1989) produced a 74\% correlation between total reactive Al and DOC from brown water streams impacted by forestry conversion, again suggesting a strong $\mathrm{Al}$ affinity for HS.

\subsubsection{HS Application to Dissolved Metal Removal}

This section builds on the previous discussion by citing from the literature examples of quantitative metal removal from the dissolved fraction. There is relatively little published data using HS for treatment of metal contaminated water. Therefore, discussion based on metal removal trials by sorption using lignite (the parent material of Solid Energy Humic Acid (SEHA)) has been included.

\subsubsection{Lignite Derived HS Complexation Capacity}

Cezikova et al. (2001) derived binding coefficients using a lignite derived HS for a range of metals, which included the full target metal suite. At pH 4.5, the complexation capacity of $\mathrm{HS}$ for $\mathrm{Pb}, \mathrm{Cd}, \mathrm{Cu}, \mathrm{Zn}$ and $\mathrm{Ni}$ was 45.5, 15.7, 13.3, 5.23, 4.7 mg/g (Čežíková, Kozler et al. 2001), suggesting a molar hierarchy for HS sorption capacity of $\mathrm{Pb}>\mathrm{Cu}>\mathrm{Cd}>\mathrm{Ni}$ $\sim$ Zn. Pehlivan and Arslan (2007) performed similar experiments using 0.15mm lignite particles to remove $1 \mathrm{mM} \mathrm{Cu}, \mathrm{Pb}$ and $\mathrm{Ni}$ by adsorption in batch reactions. Maximum removal was achieved at $\mathrm{pH} 4.5$, with $\mathrm{Cu}, \mathrm{Pb}$ and $\mathrm{Ni}$ adsorption capacities of 62.6, 18.4 and $12.5 \mathrm{mg} / \mathrm{g}$, respectively. Pehlivan and Arslan (2007) showed the coal with the highest oxygen and functional group content had the greatest metal complexation capacity. The authors suggested that at low $\mathrm{pH}(<2)$ all functional groups on the coals were protonated, and thus hydrogen ions outcompeted metals for binding sites.

Jochová et al. (2004) used a $\mathrm{Ca}(\mathrm{OH})_{2}$ activated lignite in dissolved $\mathrm{Zn}$ removal experiments. A series of batch experiments established a lignite absorption capacity of approximately $50 \mathrm{mg} / \mathrm{g}$, after 24 days. Column tests showed that sorption kinetics were relatively slow, hence long column residence times required to avoid premature breakthrough.

Havelcová et al. (2009) performed batch experiments investigating the $\mathrm{Pb}, \mathrm{Zn}, \mathrm{Cu}$ and $\mathrm{Cd}$ binding capacity of HS using products at different stages of extraction, including a raw lignite, alkali humin (filtrand after alkali extraction) and HA (acidified filtrate after alkali 
extraction). The alkali humin resulted in the greatest adsorption of metals, followed by lignite, then HA. The authors suggested the additional metal removal achieved by alkali humin was due to the initial deprotonation of carboxylic groups resulting in increased sorption capacity.

\subsubsection{Application of HS}

\subsubsection{Process considerations}

The incorporation of HS complexed metals into settling floc is critical in increasing the total removal of metals. Interactions between HS macromolecules via cationic bridges, polar interactions, hydrogen bonding or van der Waals forces may cause HS insolubility (Swift 1996), resulting in removal of HS complexed metals by sedimentation. Similar HS binding mechanisms may occur with precipitated metal hydroxide surfaces (Warren and Zimmerman 1994), resulting in adsorption of HS complexed metals to precipitates, and subsequent enhanced settling.

In an HS dosed AMD neutralisation scenario, HS metal complexation and oxide adsorption would occur simultaneously. Warren et al. (1994) investigated the competition between suspended particulate iron oxides $(\mathrm{FeOx})$ and organic matter for adsorption/complexation of $\mathrm{Cd}, \mathrm{Zn}$ and $\mathrm{Cu}$. They showed the adsorption densities of $\mathrm{FeOx}$ for $\mathrm{Cd}$ and $\mathrm{Zn}$ were 1-2 orders of magnitude greater than DOM at circum-neutral pH. Overall DOM had a greater $\mathrm{Cu}$ adsorption density than $\mathrm{FeOx}$, however trials adding particulate $\mathrm{FeOx}$ to $\mathrm{DOM}$-complexed $\mathrm{Cu}$ showed $\mathrm{FeOx}$ was an effective scavenger, decreasing the proportion of $\mathrm{Cu}$ complexed to DOM. Alternate trials adding DOM to FeOx-sorbed $\mathrm{Cu}$ resulted in an increase in $\mathrm{Cu}$ sorption, suggesting $\mathrm{FeOx-DOM}$ complexation increased the system sorption capacity for $\mathrm{Cu}$ (Warren and Zimmerman 1994). Thus, HS incorporation in AMD treatment may result in an increased sorption capacity of precipitated hydroxides for some metals due to additional DOM complexation.

Intermolecular bridging of $\mathrm{HA}$ by $\mathrm{Ca}$ complexation resulted in rapid formation and growth of HA aggregates at circum neutral pH (Chen and Elimelech 2007). Chen et al. (2007) showed that $\mathrm{HA}$ aggregation by $\mathrm{Na}$ and $\mathrm{Mg}$ was negligible, even at high concentrations. As $\mathrm{Na}$ is monovalent, intermolecular bridging would not be possible. Thus, addition of $\mathrm{HS}$ in $\mathrm{Ca}$ based alkalinity neutralisation of AMD may enhance sedimentation. 


\section{Literature Review}

Sorption of HS onto calcite increases slightly at HS concentrations up to $15 \mathrm{mg} / \mathrm{L}$ and rapidly at greater HS concentrations (Lee, Elzinga et al. 2005). Thus, adsorption to calcite at higher HS doses may result in enhanced HS complexed metal removal.

\subsubsection{Design of HS}

Application of HS to remediation technologies remains limited for two reasons. Firstly, few natural HS possess the reactive properties required to treat selected contaminants. Secondly, HS are polydisperse and heterogeneous, and therefore vary between sources and the extraction methods used by suppliers (Perminova and Hatfield 2005).

Extraction/modification of more uniform humic materials involves reducing the structural heterogeneity and polydispersity. Structural enhancement increases the number of desirable reactive functional groups. Polydispersity decreases where monomer addition forms bridges between humic particles, resulting in larger particles with better flocculating characteristics (Perminova and Hatfield 2005).

Kholodov et al. (2004) investigated the potential to enhance the detoxifying ability of HS through enrichment with hydroquinoic and catechol moieties.
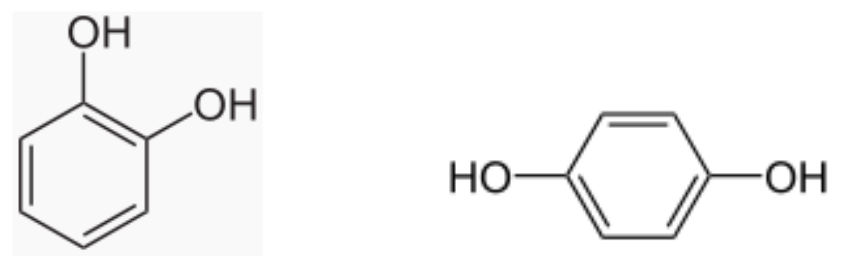

Figure 2-4: Catechol and hydroquinoic moiety

Both catechol and hydroquinone enhanced HS had three times the number of phenolic groups, per gram, at approximately $4 \mathrm{mmol} / \mathrm{g}$ (Kholodov, Kovalenko et al. 2004). Thus, the modified HS would have a greater complexation capacity for metals which favour phenolic sites. 


\subsection{Lignite-derived HS for AMD Remediation}

This literature review has shown the interaction of HS with metals in both the natural and lab environment. The complexation of metals by HS is variable and controlled by a range of variables specific to a given scenario.

Of the existing publications reviewed for this thesis, none

- $\quad$ used alkali extracted ( $\mathrm{pH} \sim 10-12)$ liquid phase HS for metal recovery; or

- used raw AMD as a stock metal solution.

This thesis is significant as it aims to apply HS to the engineered treatment of AMD. The initial deprotonation of HS functional groups at alkali $\mathrm{pH}$ theoretically enhances the capacity for neutralisation of acidity and complexation of dissolved metals. Addition of a liquid HS should result in a high degree of mixing, maximising HS and metal interaction and potential complexation. Thus, use of a liquid $\mathrm{HS}$ at alkali $\mathrm{pH}$ would maximise the reactivity of HS. The interaction between HS and hydroxide precipitates should result in removal of HS complexed metals by sedimentation.

The complex chemistry of raw AMD introduces a range of phenomena, which may enhance or inhibit the complexation of metals by HS. These phenomena may include binding competition from other metals/electrolytes for HS complexation sites, adsorption to metal hydroxide $\left(\mathrm{Fe}(\mathrm{OH})_{3}, \mathrm{Al}(\mathrm{OH})_{3}\right)$ and other mineral sites and complexation with non-organic ligands (i.e. $\mathrm{SO}_{4}{ }^{2-}$ ). Thus, the use of $\mathrm{HS}$ complexation affinities for metals quoted from the literature may be unreliable for AMD applications. 


\section{Chapter 3. Methodology}

The methodology chapter is divided into three sections.

Section 1: Setting. The BTP and MSS neutralisation systems are put into context locally within the mine site and on a larger scale within the Ngakawau River catchment. The sample collection point is also identified.

Section 2: Characterisation. Characterisation was required to improve understanding of the specific HS under investigation. Effective characterisation provides a basis for comparison of the HS used in this project with HS used in the literature. Characterisation of HS and AMD sludge are described.

Section 3: Laboratory Methodologies. Most laboratory trials used modified jar testing procedures where mixing conditions were changed to represent incorporation of HS dosing in the Blackwater Treatment Plant (BTP) and the Mangatini Stream-sump System (MSS) treatment systems. A range of parameters were measured for treated samples including $\mathrm{pH}$, turbidity, suspended solids (SS) and dissolved metal concentration. These parameters were used to describe the water quality and metal removal efficiency of various treatment scenarios. This section also includes the methodology used to investigate $\mathrm{HS}$ as a polymer and the equilibrium speciation modelling. 
Methodology

\subsection{Setting}

The Blackwater Treatment Plant (BTP) and Mangatini Stream-sump (MSS) are two separate AMD treatment systems located at the Stockton Mine on the West Coast of New Zealand. The BTP treats AMD piped from the Stockton Mine coal processing areas and has a greater proportion of coal fines. The MSS treats the Upper Mangatini Stream, which is impacted by AMD. The BTP discharges into the Lower Mangatini Stream, below the Mangatini Sump. The Mangatini Stream eventually flows into the Ngakawau River and out to the Tasman Sea. Figure 3-1 shows schematically the BTP and MSS and their proximity to the Ngakawau River.

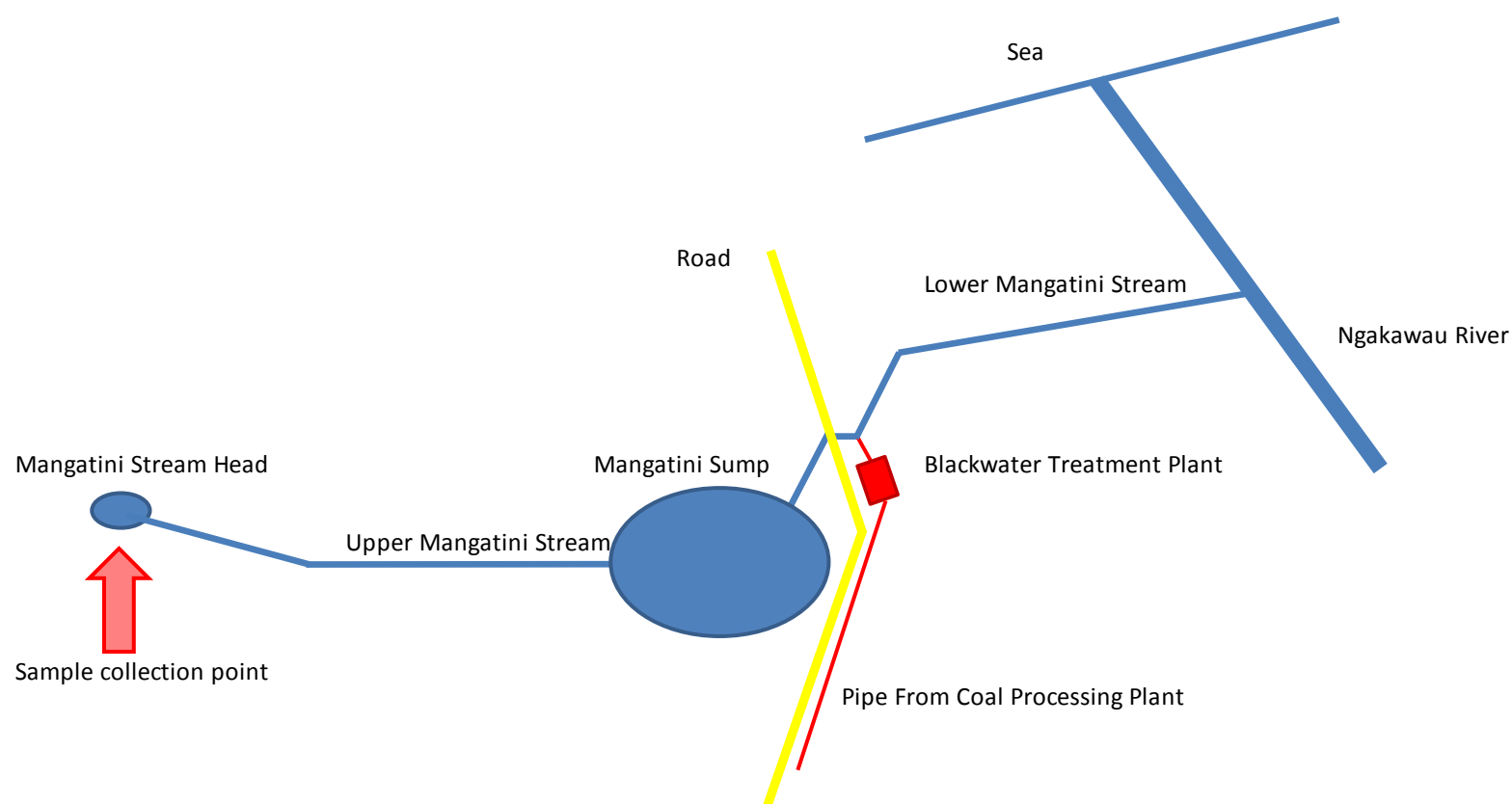

Figure 3-1: Schematic of relevant Water bodies

The BTP is an active AMD neutralisation treatment plant. Acid mine drainage flows into the treatment plant where it is rapidly neutralised by flash mixing with a hydrated lime $\left(\mathrm{Ca}(\mathrm{OH})_{2}\right)$ slurry. The neutralised AMD then enters a paddle flocculation tank where slow mixing promotes floc formation. At the outlet of the flocculation tank a polymer is added before the neutralised AMD enters a lamella clarifier where floc settles out and forms sludge. The design residence time in both the flocculation tank and clarifier was 30 minutes. The supernatant is discharged to the Lower Mangatini Stream while the sludge is extracted and dewatered by polymer assisted centrifuge. The dewatered sludge is stored and later disposed of onsite. 
Methodology

The MSS system is also an active AMD neutralisation system, which treats the Upper Mangatini Stream (UMS). The UMS drains working areas of disturbed acid generating rock. Therefore the UMS is acidic and classified as AMD. The head of the UMS is continuously dosed with ultra-fine limestone $\left(\mathrm{CaCO}_{3}\right)$ slurry. Approximately one hour of turbulent in-stream mixing provides sufficient residence time for neutralisation to $\mathrm{pH}$ 6-7. The stream discharges into the man-made Mangatini Sump which has an estimated residence time for sedimentation of up to seven days; although during significant rainfall events the residence time would be decreased as stream flow rates vary by up to two orders of magnitude (Davies, Weber et al. 2011b). Over the sedimentation period, precipitated metal hydroxides, sediment derived from erosion, and unreacted $\mathrm{CaCO}_{3}$ settle out, forming sludge. The supernatant is discharged over a weir into the lower Mangatini Stream. The sludge accumulates in the sump, and will eventually require excavation after a number of years of operation. The utilisation of turbulent in-stream mixing combined with the absence of mechanically induced flocculation give the MSS some passive treatment characteristics, although continuous chemical dosing is typical of an active treatment scenario (Trumm 2010).

\subsubsection{Sample Collection}

Raw AMD was collected from the Stockton Mine on the West Coast of New Zealand at the sample collection point shown on Figure 3-1. The sample collection point was a small pond at the top of the UMS. The AMD collected would therefore have flowed into the MSS treatment system. The same AMD source was used for both the BTP and MSS experiments.

Due to site topography it was not possible to get a vehicle close to the sampling point. Therefore approximately 600L of AMD was collected in 20L plastic tanks, which were rinsed with AMD before sample collection. The tanks were transported back to the University of Canterbury where they were poured into a new 1000L Intermediate Bulk Container (IBC) to homogenise.

As AMD was required it was sub-sampled from the IBC into a $20 \mathrm{~L}$ plastic tank using a submersible pump. By subsampling using a pump, material which had settled to the bottom of the tank was not re-suspended and used in experiments. 


\subsection{Characterisation}

Characterisation of HS and AMD sludge was performed to show the abundance of different reactive sites for both metal complexation and adsorption.

\subsubsection{Humic Substance}

A range of characterisation tests were performed on the HS. These include CHON, Fourier Transform Infrared spectroscopy (FTIR) and solid-state ${ }^{13} \mathrm{C}$ Nuclear Magnetic Resonance (NMR) analysis. The CHON analysis identified the elemental composition of HS. Analysis by FTIR and NMR identified the presence and distribution of carbon containing functional groups, allowing characterisation as fulvic/humic acid. These properties were used to compare SEHA with other characterized HS in the literature. The characterisation of W7 lignite (parent material of SEHA) provided by SENZ was included to show the changes in HS structure that occur due to alkali extraction.

\subsubsection{CHON}

$\mathrm{CHON}$ and ash analysis is a destructive technique used to determine the carbon, hydrogen, oxygen, nitrogen, sulphur and ash content of a material. CHON analysis is a common characterisation procedure used by most HS researchers (Gao, Pearce et al. 1999; Monteil-Rivera, Brouwer et al. 2000; Novak, Kozler et al. 2001; Weber, Tang et al. 2006; Pehlivan and Arslan 2007; Havelcová, Mizera et al. 2009).

The CHON analysis was performed by the Chemistry Department at the University of Otago using an Elementar (Hanau, Germany) Combustion Analyser. The results were given as percent weight (wt\%) for each element.

\section{2 .1 .2 FTIR}

Fourier-transform Infrared (FTIR) spectroscopy provides qualitative information about the structural and functional groups of HS. Dry samples of SEHA and AHA were analysed using a Perkin Elmer (Massachusetts, USA) SpectrumOne FTIR. The SEHA sample was prepared by slowly drying the raw liquid SEHA at below $40^{\circ} \mathrm{C}$. The dry samples were ground to a powder and mixed with Potassium Bromide $(\mathrm{KBr})$. A range of sample to $\mathrm{KBr}$ ratios $(0.5-2 \%)$ were used to optimise peak detection. The samples were blanked against $\mathrm{KBr}$ and then the data were exported as absorbance at the wavelength of $600-4000 \mathrm{~cm}^{-1}$ for later analysis. FTIR analysis was performed in the Chemistry Department of the University of Canterbury. 
The absorbance at particular wavelength bands corresponds to detection of different bonds. Major groups identified by FTIR were hydroxyl $\left(3600-3200 \mathrm{~cm}^{-1}\right)$, aliphatic C

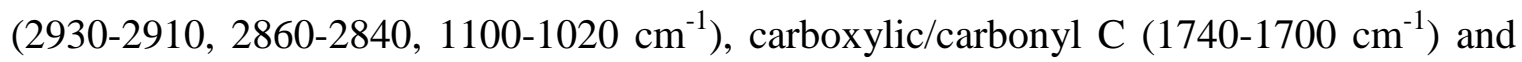
aromatic C (1615-1580, 1515-1480 $\mathrm{cm}^{-1}$ ) (Chen, Gu et al. 2002; Weber, Tang et al. 2006).

\subsubsection{NMR Spectroscopy}

Nuclear magnetic resonance (NMR) spectroscopy provides quantitative structural information about organic matter. The W7 lignite and HS samples were prepared for NMR analysis by acid washing, using a similar method to Salati et al. (2008). A $0.5 \mathrm{~g}$ sample was put in a centrifuge tube with $40 \mathrm{~mL}$ of $1 \mathrm{M} \mathrm{HCl}$. The samples were then shaken at $200 \mathrm{rpm}$ on an orbital shaker for 48 hours after which the samples were centrifuged and the supernatant poured off. The washing process was repeated ten times. The $\mathrm{HCl}$ solution from the $10^{\text {th }}$ washing was analysed by ICP-MS to show minimal residual iron leaching. Samples were then dried at less than $40^{\circ} \mathrm{C}$ and sent for analysis.

NMR experiments were undertaken in a Bruker (Rheinstetten, Germany) AMX 200Mhz horizontal bore magnet at Massey University, Palmerston North. Samples ranging from 0.26 to 0.29 grams were packed into $7 \mathrm{~mm}$ diameter Doty MAS rotors and spun at speeds of $5.0 \pm 0.3 \mathrm{kHz}$ in a dual resonance magic angle-spinning (MAS) probe from Doty Scientific. During acquisition the sample temperature was maintained at $20^{\circ} \mathrm{C}$.

The ${ }^{13} \mathrm{C}$ CP-MAS spectra were acquired with a ${ }^{1} \mathrm{H} 90^{\circ}$ pulse of $5.5 \mu \mathrm{s}$, a cross-polarisation contact time of $900 \mu \mathrm{s}$, an acquisition time of $20 \mathrm{~ms}$ and relaxation time of $1 \mathrm{sec}$. Scans were either at $8 \mathrm{k}$ or $16 \mathrm{k}$ accumulations, depending on the carbon concentration of the sample to achieve a satisfactory signal-to-noise ratio. Spectra were processed with MestReNova (ver 6.03) NMR processing Software (Mestrelab Research Ltd, Spain). All ${ }^{13} \mathrm{C}$ spectra were referenced externally against glycine.

The integrated area under peaks between specific spectral bands correlates to the distribution of structural elements of HS. Structures identified were Aliphatic (0-50 ppm), Carbohydrate/Heteroaliphatic (50-100 ppm), Aromatic (100-140 ppm), Phenolic (140165 ppm) and Carboxylic (165-190 ppm) (Monteil-Rivera, Brouwer et al. 2000; Chen, Gu et al. 2002; Weber, Tang et al. 2006). 
Methodology

\section{2 .2 AMD}

Characterisation of AMD sludge was undertaken to show the incorporation and speciation of metals (particularly $\mathrm{Fe}$ and $\mathrm{Al}$ ) in sludge. Characterisation of AMD sludge therefore shows both the fate of metals after neutralisation and identifies formation of $\mathrm{Fe}$ and $\mathrm{Al}$ oxides capable of trace metal removal by adsorption.

\subsubsection{FTIR}

Fourier-transform Infrared spectroscopy can also be used to identify mineral groups. Samples of AMD sludge were prepared for FTIR analysis by drying at below $40^{\circ} \mathrm{C}$. The $\mathrm{KBr}$ method was used to analyse AMD sludge, as described in section 3.2.1.2.

The absorbance of inorganic carbonates and oxides occurs at different wavelengths. Absorbance of clay minerals and oxides occurs at 3750-330, 1200-970, and 950-820 cm ${ }^{-1}$ while absorbance by carbonates occurs at 1600-1300, 1600-850, and 900-670 $\mathrm{cm}^{-1}$ (Johnston and Aochi 1996).

\section{$3.2 .2 .2 \mathrm{XRF}$}

X-ray Fluorescence (XRF) analysis was used to determine the elemental composition of AMD sludge. The XRF was performed by SpectraChem Analytical of CRL Energy Ltd. Elemental composition was determined by powder briquette X-ray spectrometry. Loss on ignition was determined gravimetrically.

All detected elements were assumed to occur as oxides, except for $\mathrm{Fe}, \mathrm{Al}$, and $\mathrm{Ca}$. Both Fe and $\mathrm{Al}$ were expected to occur as hydroxides $\left(\mathrm{Fe}(\mathrm{OH})_{3}\right.$ and $\left.\mathrm{Al}(\mathrm{OH})_{3}\right)$. Due to the low limestone dissolution, all $\mathrm{Ca}$ was assumed to occur as $\mathrm{CaCO}_{3}$. This assumption was verified by XRD analysis which showed high amounts of $\mathrm{CaCO}_{3}$ in $\mathrm{AMD}$ sludge neutralised by $\mathrm{CaCO}_{3}$.

\section{$3.2 .2 .3 \quad X R D$}

X-ray Diffraction (XRD) was used to determine the abundance and speciation of crystalline mineral groups present in AMD sludge. The XRD was also performed by SpectraChem using a Philips PW1720 X-ray Diffractometer. The XRD analysis was also performed by CRL Energy Ltd. 


\subsection{Lab Methodologies}

This section describes the lab experimental methodologies. General methodologies are described first. The method for determining the neutralisation dose for BTP $(\mathrm{NaOH}$, $\left.\mathrm{Ca}(\mathrm{OH})_{2}\right)$ and $\mathrm{MSS}\left(\mathrm{CaCO}_{3}\right)$ is described second. The third section describes the HS dosing and mixing conditions for jar testing. The fourth section describes how supernatant samples were collected for water quality and metal removal analysis. Finally, the speciation modelling method is discussed.

\subsubsection{General Methodologies}

The general methods described in this section were used throughout the experimental part of the project. They are described here and then referenced in the following sections where applicable.

\subsubsection{Stock Solution}

Stock solutions were prepared for neutralisation of AMD and HS dosing. The benefit of using stock solutions was that alkalinity and HS dosing could be done volumetrically using a pipette which was faster than weighing numerous dry samples.

\subsection{Alkalinity Reagents}

A stock solution of $1 \mathrm{M} \mathrm{NaOH}$ was prepared using laboratory grade sodium hydroxide pallets. A $40 \mathrm{~g}$ sample of $\mathrm{NaOH}$ pallets was weighed into a $1 \mathrm{~L}$ volumetric flask approximately $80 \%$ filled with deionised water. The flask was placed in a cool water bath (to absorb the heat released by the exothermic $\mathrm{NaOH}$ dissolution) on a magnetic stirrer. The flask contents were stirred rapidly until all $\mathrm{NaOH}$ pallets had dissolved. The magnetic flea was then removed and the solution made up to $1 \mathrm{~L}$ by deionised water.

A stock slurry of $1 \mathrm{M} \mathrm{Ca}(\mathrm{OH})_{2}$ was prepared using laboratory grade calcium hydroxide powder. A $74 \mathrm{~g}$ sample of $\mathrm{Ca}(\mathrm{OH})_{2}$ was weighed into a $1 \mathrm{~L}$ volumetric flask approximately $80 \%$ filled with deionised water. The flask was vigorously shaken to homogenise, then made up to $1 \mathrm{~L}$ with deionised water. The solubility of $\mathrm{Ca}(\mathrm{OH})_{2}$ at room temperature is approximately $1.85 \mathrm{~g} / \mathrm{L}$ (CRC 1976), so the slurry was significantly supersaturated.

A stock slurry of $1 \mathrm{M} \mathrm{CaCO}_{3}$ was prepared from sample of fine limestone $\left(\mathrm{CaCO}_{3}\right)$ used at the Stockton Mine. A $100 \mathrm{~g}$ sample was weighed into a $1 \mathrm{~L}$ volumetric flask, which was then $80 \%$ filled with deionised water. The flask was then vigorously shaken to 
Methodology

homogenise and made up to $1 \mathrm{~L}$ with deionised water. The solubility of $\mathrm{CaCO}_{3}$ at room temperature is approximately $0.014 \mathrm{mg} / \mathrm{L}$ (CRC 1976), meaning the slurry was significantly supersaturated.

The neutralisation of AMD by stock solutions of $\mathrm{Ca}(\mathrm{OH})_{2}$ and $\mathrm{CaCO}_{3}$ was not possible due to low solubility at circum-neutral $\mathrm{pH}$. Theoretically, a total of $350 \mathrm{~mL}$ of $\mathrm{Ca}(\mathrm{OH})_{2}$ and $130 \mathrm{~L}$ of $\mathrm{CaCO}_{3}$ stock solution (with concentration at saturation limits) would be required to provide the necessary alkalinity to neutralise $1 \mathrm{~L}$ of AMD; calculated from the alkalinity associated with the dissolved fraction of $\mathrm{CaCO}_{3}$, not the unreacted fraction which would dissolve in acidic solutions. Thus, volumetric dosing using a pipette from the stock slurry with vigorous shaking before extraction to homogenise particulate was used. This method was improved towards the end of experimentation by continuously stirring the slurry using a magnetic stirrer and extracting the alkalinity dose by pipette. The maximum dose volume of $18 \mathrm{~mL}$ for $\mathrm{CaCO}_{3}$ neutralisation resulted in minimal dilution $(<1.8 \%)$ of metals.

The purity of the Stockton $\mathrm{CaCO}_{3}$ was determined by the EDTA titrimetric method described in the APHA standard methods textbook (APHA 2005). The EDTA was standardised against a standard calcium solution prepared from laboratory grade $\mathrm{CaCO}_{3}$ powder. The Stockton $\mathrm{CaCO}_{3}$ was determined to be approximately $99 \mathrm{wt} \%$ pure by this method.

All alkalinity stock solutions were stored in Schott bottles out of direct sunlight and shaken vigorously before use.

\subsection{HS Solutions}

The Aldrich Humic Acid (AHA) stock solution was prepared from dry AHA powder sourced from Sigma-Aldrich. A $500 \mathrm{mg}$ sample of dry AHA was weighed and then put into a $1 \mathrm{~L}$ volumetric flask. The AHA was dissolved using $40 \mathrm{~mL}$ of $1 \mathrm{M} \mathrm{NaOH}$. Approximately $800 \mathrm{~mL}$ of deionized water was added, and the contents stirred on a magnetic stirrer for 48 hours. The solution was then made up to $1 \mathrm{~L}$ and filtered through a $0.45 \mu \mathrm{m}$ filter. The resulting filtrate was kept for experimentation as a nominal $500 \mathrm{mg} / \mathrm{L}$ dissolved AHA. Addition of $40 \mathrm{~mL}$ of the stock AHA solution to $1 \mathrm{~L}$ of AMD resulted in a nominal HS concentration of $20 \mathrm{mg} / \mathrm{L}$ AHA. Stock solutions of AHA were stored at less than $4{ }^{\circ} \mathrm{C}$ in Schott bottles wrapped in tin foil to exclude light. 
Methodology

The Solid Energy Humic Acid (SEHA) comprised both humic and fulvic acids. The SEHA was derived by caustic extraction of W7 lignite. Lignite $(<500 \mu \mathrm{m})$ was placed inside a digester with $0.5 \mathrm{M} \mathrm{NaOH}$ at a solid to liquid ratio of 1:5. The mixture was stirred for half an hour before heating to $90{ }^{\circ} \mathrm{C}$. The mixture was then digested for two hours at $90{ }^{\circ} \mathrm{C}$ before being shut down and allowed to cool over-night. The liquid fraction (SEHA) was then separated by centrifugation.

As a product of caustic extraction, the SEHA had a high $\mathrm{pH}(\sim 10)$, resulting in deprotonation of functional groups. A stock solution of the SEHA was not prepared as the initial deprotonation of SEHA was favourable to both acid neutralisation and metal complexation. The SEHA product was stored in a non-transparent plastic bottle out of direct sunlight.

The liquid SEHA had a concentration of $66,960 \mathrm{mg} / \mathrm{L} \mathrm{HS}$, determined by SENZ, using ISO 5073:1999 (ISO 1999). Therefore addition of 0.30 and $1.49 \mathrm{~mL}$ of the liquid HS to 1 L AMD resulted in HS concentrations of 20 and $100 \mathrm{mg} / \mathrm{L}$, respectively.

Stock solutions of $0.1 \%$ Crystalfloc B570 and B610 were prepared for dosing in the polymer trials. A $0.5 \mathrm{~g}$ sample of polymer was weighed into a $500 \mathrm{~mL}$ volumetric flask. The flask was then $80 \%$ filled with deionised water and placed on a magnetic stirrer heating plate. The flask was stirred and gently heated for 45-60 minutes until all polymer had dissolved. The stock solution was then made to volume and stored in a Schott bottle. The polymer solution was used within two days of preparation, or discarded if polymer precipitation was observed.

\subsubsection{2 pH Measurement}

The $\mathrm{pH}$ of samples was measured using an EDT Instruments RE357Tx $\mathrm{pH}$ meter. The $\mathrm{pH}$ meter was calibrated using $\mathrm{pH} 4$ and 7 standards, according to the manufacturer's instructions. The calibration was checked before use and recalibrated if necessary.

Instrument readings of $\mathrm{pH}$ in the circum-neutral range took a few minutes to stabilise. To accelerate stabilization, samples were stirred at a moderate speed by magnetic stirrer during measurement. The equilibrium $\mathrm{pH}$ was then recorded as the sample $\mathrm{pH}$.

\subsubsection{Turbidity Measurement}

The turbidity was measured using a Hach 2100 turbidimeter. The calibration was checked using Gelex ${ }^{\circledR}$ secondary turbidity standards in the $0-10,0-100$, and 0-1000 NTU ranges. 
Methodology

A $10 \mathrm{~mL}$ supernatant sample was extracted from $10 \mathrm{~mm}$ below the water surface by syringe and transferred to a turbidity vial without filtration. The outside surfaces of the vial were then cleaned and placed inside the meter for measurement.

\subsubsection{SS Measurement}

Supernatant SS concentrations were relatively low. Therefore a $500 \mathrm{~mL}$ sample was used to maximise the measured SS mass and reduce the detection limit. Supernatant was gently poured (to prevent re-suspension of sludge) from the testing beaker into a $500 \mathrm{~mL}$ measuring cylinder. Samples were filtered through dry pre-weighed Whatman glass-fibre (GF/C) filter paper, with nominal particle retention of $1.2 \mu \mathrm{m}$ (Whatman 1999). The filter paper was then dried for at least one hour at $105{ }^{\circ} \mathrm{C}$. The samples were then re-weighed and the SS was determined using Equation 3-1:

$$
S S=\frac{M_{f}-M_{i}+M_{o}}{2000}
$$

Equation 3-1

Suspended solids equation

Where, $M_{f}$ was the final mass of the filter paper $\mathrm{M}_{\mathrm{i}}$ was the initial mass of the filter paper $\mathrm{M}_{\mathrm{o}}$ was the mass of glass fibre washed out of the filter paper by $500 \mathrm{~mL}$ deionised water; $0.0013 \mathrm{~g}$ on average.

\subsubsection{UV-Vis Measurement}

The residual HS concentration in filtered samples was measured using a Thermoscientific Genesys 10S UV-Vis spectrophotometer and quartz vials. An absorbance calibration curve was developed using dilutions of SEHA from 100 to $0.2 \mathrm{mg} / \mathrm{L}$, blanked against deionised water. The absorbance of diluted SEHA standards was measured at 254 and $280 \mathrm{~nm}$, where absorbance by HS is high (Chen, Gu et al. 2002). The calibration curve (Figure 3-2) was constructed by plotting adsorbance against known HS concentration. 


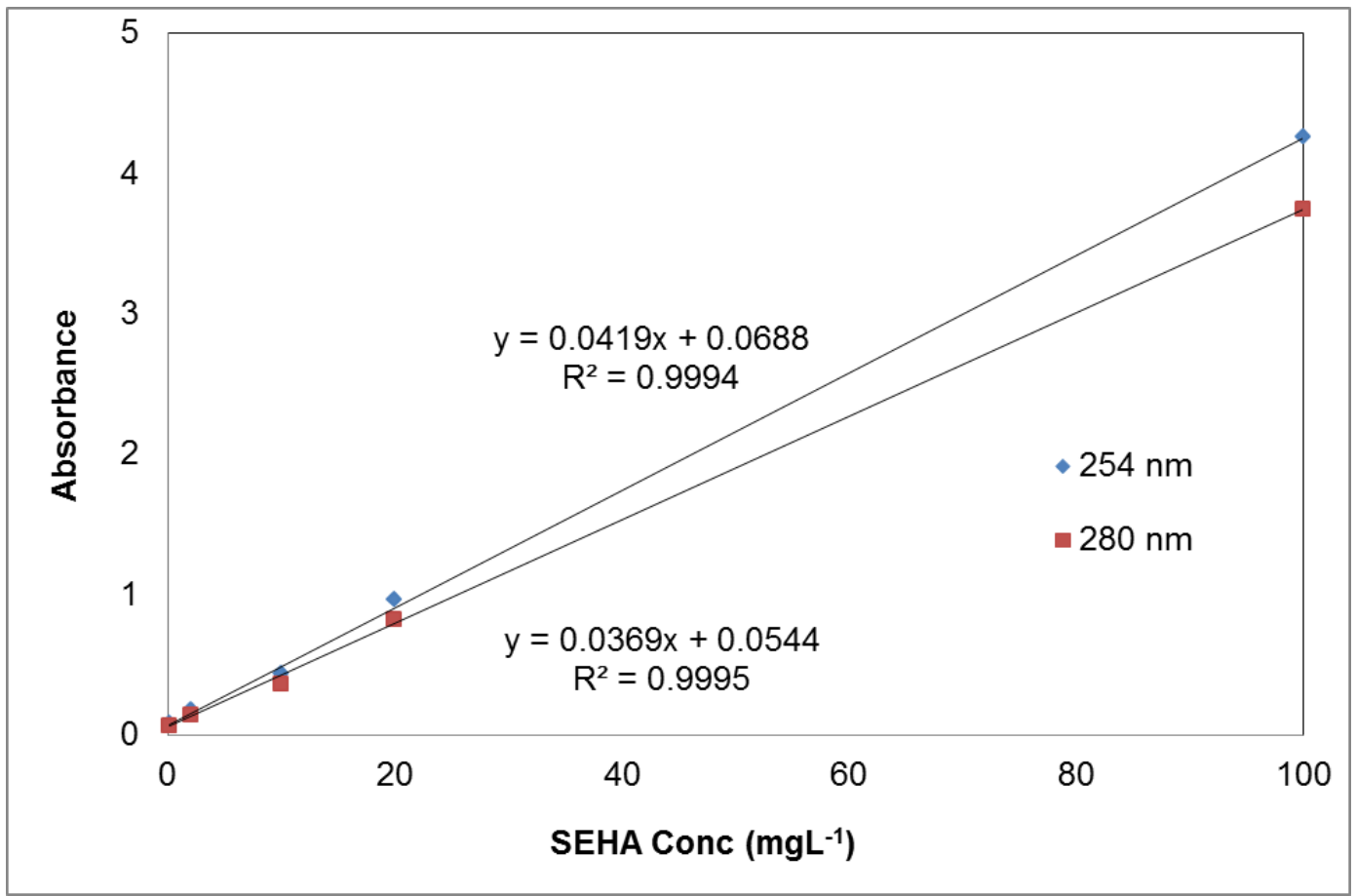

Figure 3-2: Calibration curve for UV-Vis measurement of HS concentration

Sample absorbance was also blanked against deionised water. Vials were rinsed with deionised water between samples and the vial faces cleaned before measurement. Samples with absorbance over 3.0 were diluted as the HS-absorbance relationship became non-linear. The absorbance of samples was converted to a HS concentration using the calibration curve.

\subsubsection{ICP-MS Measurement}

The concentration of a suite of metals was determined using an Agilent (California, USA) 7500cx Inductively Coupled Plasma Mass Spectrometry (ICP-MS) instrument, under standard hot plasma conditions. Before running samples, standards at 0, 0.1, 1, 10, 100 and $1000 \mu \mathrm{g} / \mathrm{L}$ were used to check the range of the instrument. Every tenth sample was duplicated and every $20^{\text {th }}$ spiked as a quality control measure. Blanks were run after spikes before further samples were analysed.

The theoretical detection limits published by Agilent for the 7500cx ICP-MS were extremely low, between 0.27 and 3.1 parts per trillion (ppt) under idealised analysis conditions (Wilbur 2007). For this project, the instrument detection limits were determined using the three sigma method. The three sigma limits were calculated by multiplying the standard deviation of the reported metal concentration of blank samples by three. The instrument detection levels were typically two orders of magnitude greater 
Methodology

than the idealised instrument detection levels published by Agilent, and are shown on Table 3-1.

Table 3-1: Detection limits of target metals for this thesis

\begin{tabular}{ccc}
\hline Metal & $\begin{array}{c}\text { Instrument Detection Limit } \\
(\mathbf{u g} / \mathbf{L})\end{array}$ & $\begin{array}{c}\text { Experiment Detection Limit } \\
(\mathbf{m g} / \mathbf{L})\end{array}$ \\
\hline $\mathbf{N i}$ & 0.067 & 0.007 \\
$\mathbf{Z n}$ & 0.143 & 0.027 \\
$\mathbf{C u}$ & 0.272 & 0.014 \\
$\mathbf{C d}$ & 0.011 & 0.001 \\
$\mathbf{P b}$ & 0.244 & 0.024 \\
\hline
\end{tabular}

The maximum concentration analysable by ICP-MS for all metals was $1 \mathrm{mg} / \mathrm{L}$. Thus, raw samples required 100 times dilution due to high $\mathrm{Na}$ and $\mathrm{Ca}$ concentrations, depending on the alkalinity reagent used. These dilutions were achieved by one step dilution; $0.1 \mathrm{~mL}$ sample to $9.9 \mathrm{~mL}$ DI water. Samples were then refrigerated for later analysis. The experimental detection limit is used in discussion throughout this thesis and was determined as 100 times the instrument detection limit.

Metal analysis using inductively coupled plasma optical emission spectroscopy (ICPOES) has a higher maximum concentration limit. Use of ICP-OES would therefore have required less dilution, which may have resulted in higher quality metal concentration data. However, ICP-OES was not available at the University of Canterbury at the time of this research. 


\subsubsection{Neutralisation}

The BTP neutralisation dose was defined as the alkalinity required to raise the $\mathrm{pH}$ of AMD to 7. The $\mathrm{NaOH}$ neutralisation dose was determined by titration, as the dissociation of $\mathrm{NaOH}$ was instantaneous. A $1 \mathrm{~L}$ measuring cylinder was filled with AMD which was then poured into a $1 \mathrm{~L}$ beaker. The AMD was stirred steadily by magnetic stirrer. A pH probe was held by clamp stand to measure the $\mathrm{pH}$ of the AMD solution. The AMD was then titrated using $0.5 \mathrm{~mL}$ increments of $1 \mathrm{M} \mathrm{NaOH}$ to over $\mathrm{pH}$. The $\mathrm{pH}$ was recorded for each increment of titrant. The $\mathrm{NaOH}$ neutralisation dose was defined as the dose to $\mathrm{pH}$ 7 by titration.

The $\mathrm{Ca}(\mathrm{OH})_{2}$ neutralisation dose was determined by jar test. The dissociation/neutralisation kinetics of $\mathrm{Ca}(\mathrm{OH})_{2}$ are rapid at acidic $\mathrm{pH}$, but slow at circumneutral $\mathrm{pH}$. Thus, $\mathrm{pH}$ tended to creep upwards (over a period of hours) when samples were rapidly neutralised (by slug dose) to $\mathrm{pH} 7$, as residual $\mathrm{Ca}(\mathrm{OH})_{2}$ dissociated. A series of $1 \mathrm{~L}$ beakers of AMD were neutralised by varying $\mathrm{Ca}(\mathrm{OH})_{2}$ doses $(6.5-10 \mathrm{~mL}$ of $1 \mathrm{M}$ $\mathrm{Ca}(\mathrm{OH})_{2}$ slurry) under standard jar test mixing conditions (1 min at $100 \mathrm{rpm}$, followed by $25 \mathrm{~min}$ at $20 \mathrm{rpm}$ ). The $\mathrm{pH}$ of the neutralised AMD was measured at the end of the 20 rpm mixing period. The $\mathrm{Ca}(\mathrm{OH})_{2}$ dose required to neutralise AMD to $\mathrm{pH} 7$ was then interpolated from these data.

The neutralisation dose of $\mathrm{CaCO}_{3}$ required for the MSS application was also determined by jar test. The MSS neutralisation dose was defined as the $\mathrm{CaCO}_{3}$ dose required to raise the $\mathrm{pH}$ of AMD to 6, after one hour mixing at $100 \mathrm{rpm}$. An endpoint of $\mathrm{pH} 6$ was selected for MSS trials as the dissociation/neutralisation kinetics of $\mathrm{CaCO}_{3}$ decrease rapidly with $\mathrm{pH}$ increase, greatly reducing the short term $\mathrm{CaCO}_{3}$ neutralisation efficiency. A series of $1 \mathrm{~L}$ beakers of AMD were neutralised by varying $\mathrm{CaCO}_{3}$ doses $\left(2-28 \mathrm{~mL}\right.$ of $1 \mathrm{M} \mathrm{CaCO}_{3}$ slurry) under modified jar test mixing conditions (60 min at $100 \mathrm{rpm}$ ); representative of Mangatini Stream residence time and turbulence. The $\mathrm{pH}$ of the neutralised AMD was measured at the end of the mixing period. The $\mathrm{CaCO}_{3}$ dose required to neutralise AMD to pH 6 was then interpolated from these data. 


\subsubsection{Jar Testing}

This section describes the HS and polymer jar testing dosing and mixing conditions. All jar testing was duplicated to investigate the variability of results. This section describes the jar testing conditions to the end of the mixing period. All supernatant sampling and parameter measurement methods for water quality and metal removal are described separately in section 3.3.4.

\subsubsection{HS Jar Testing}

The HS jar testing produced raw samples for measurement of water quality and metal removal based on the BTP and MSS treatment systems. The results of these trials are discussed independently in Chapter 6 (HS - Water Quality) and Chapter 7 (HS - Metal Removal). Figure 3-3 shows how HS jar testing fits into the overall experimental plan with the samples used for water quality and metal removal analysis.

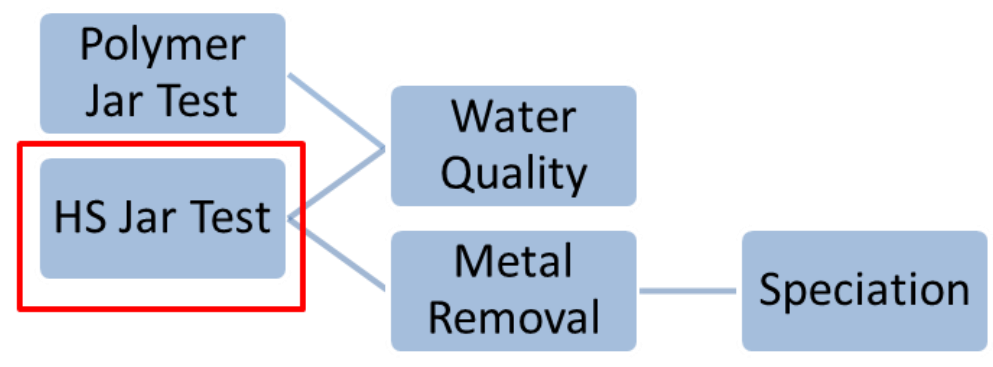

Figure 3-3: HS Jar Test context

The order of HS addition was varied to investigate the impact of background ion competition and HS co-precipitation on water quality and metal removal. Three dose orders were investigated; HS addition before-, after- and during-neutralisation. Throughout this document, the dose order "before-neutralisation" means the HS was added before neutralisation by alkalinity. Similarly, "after-neutralisation" means the HS was added after neutralisation and "during-neutralisation" means the HS was added around the neutralisation halfway point, at $\mathrm{pH} 4.5$.

The during-neutralisation condition was designed to investigate the effectiveness of HS addition to AMD approximately half way through the hydrolysis of $\mathrm{Al}$, at $\mathrm{pH} 4.5$. Theoretically, at $\mathrm{pH} 4.5$ a higher degree of HS complexation of the target metal group would occur due to reduced binding competition from $\mathrm{Al}$ and Fe. Final neutralisation to $\mathrm{pH} 7$ after $\mathrm{HS}$ dosing would trigger further $\mathrm{Al}$ floc formation (from $\mathrm{pH} 4.5-5.0$ ) to enable minor bridging and thus incorporation of HS into flock and eventually sludge. 


\subsection{BTP HS Dose and Mixing Conditions}

The HS dose conditions trialled in the $\mathrm{BTP} \mathrm{NaOH}$ neutralised jar testing experiments are shown in Figure 3-4.

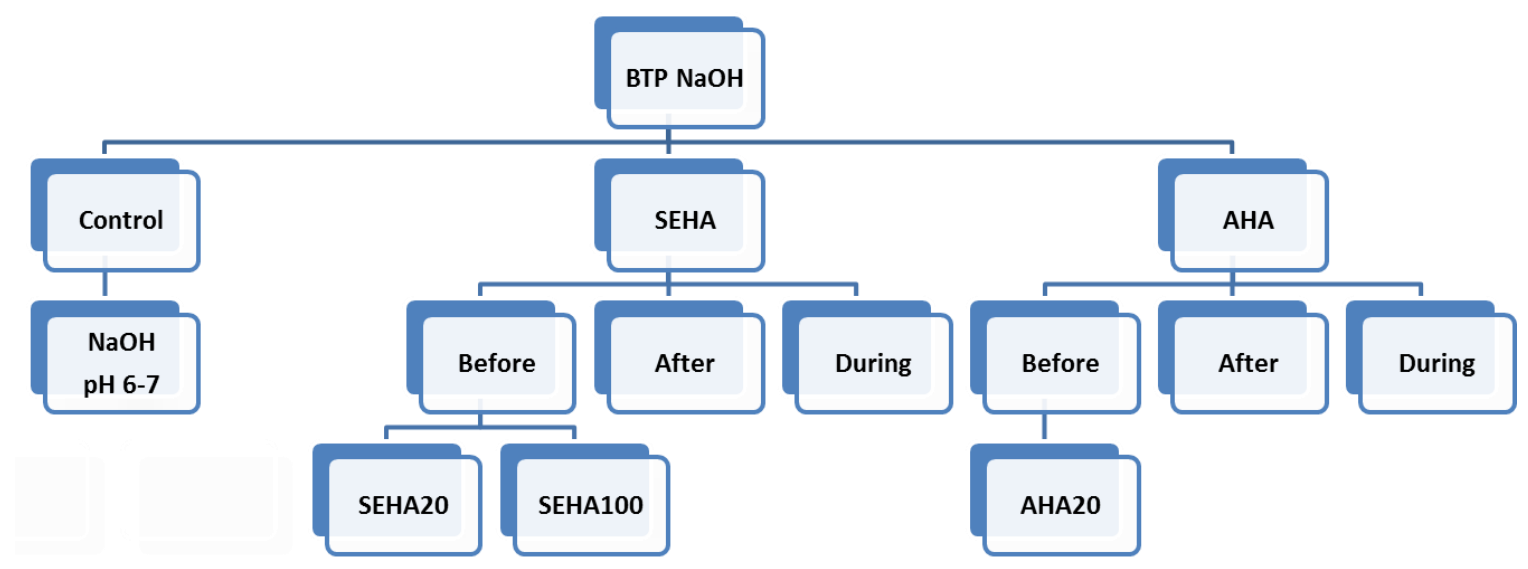

Figure 3-4: BTP conditions for $\mathrm{NaOH}$ neutralised AMD

For control samples, addition of alkalinity was followed by an initial flash mix period of 1 minute at $100 \mathrm{rpm}$. Samples were then flocculated for 25 minutes at $20 \mathrm{rpm}$, similar to the standard jar test (ASTM 2008).

A suite of control samples were prepared at approximately 0.1 increments between $\mathrm{pH} 6$ and 7 , by slightly varying the alkalinity dose of $\mathrm{NaOH}$ or $\mathrm{Ca}(\mathrm{OH})_{2}$. This meant the metal concentration of HS dosed samples could be compared with a control sample at the same $\mathrm{pH}$. This step was necessary as metal removal by different HS conditions could not be directly compared due to variation in $\mathrm{pH}$.

The HS dosing and mixing regime was varied slightly for the different HS dose orders, as shown in Figure 3-5, where rectangles represent addition of HS or alkalinity and circles represent mixing phases. 


\section{Control}

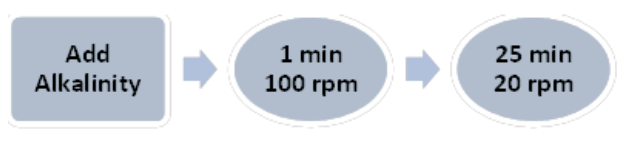

Before

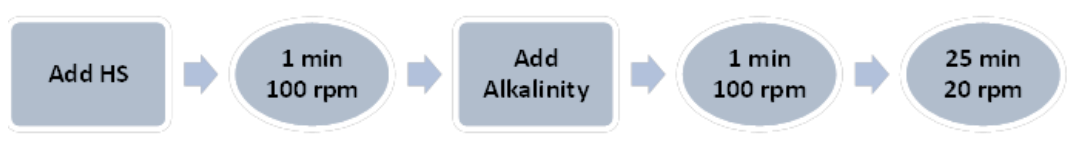

After

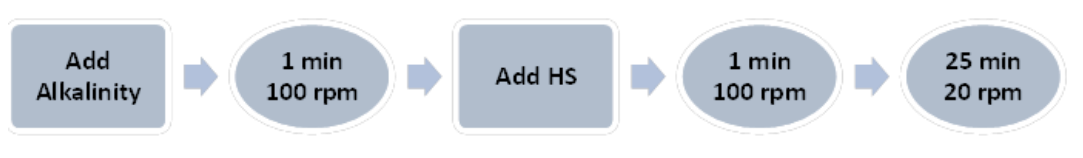

During

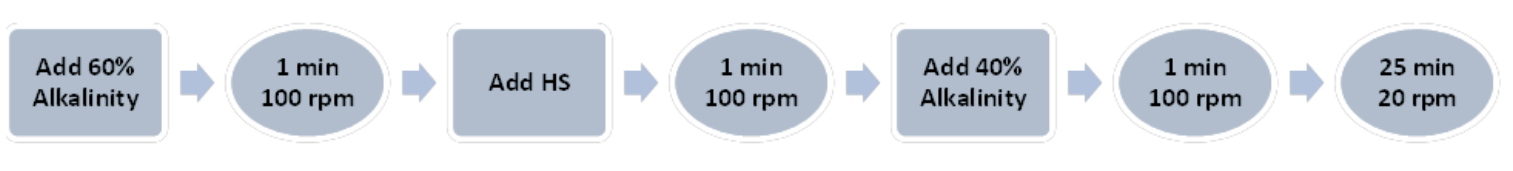

Figure 3-5: Mixing regime for $\mathrm{HS}$ dosed BTP scenario

The final mixing phase shown in Figure 3-5 corresponds to the 25 minute flocculation period. At the end of the flocculation period, the beakers were removed from the jar stir apparatus and put on a bench to allow sedimentation. The same HS dose and mixing conditions were used in the $\mathrm{Ca}(\mathrm{OH})_{2}$ neutralised trials.

\subsection{MSS Variables and Mixing Conditions}

The HS dose conditions for the $\mathrm{CaCO}_{3}$ neutralised MSS jar testing experiments were the same as shown in Figure 3-4. The mixing conditions for the MSS application were determined according to the field conditions. For control samples, one hour rapid mixing at $100 \mathrm{rpm}$ represented the residence time and turbulent flow of the Upper Mangatini Stream (UMS).

A control standard suite was also determined for $\mathrm{CaCO}_{3}$ neutralised AMD over a range of $\mathrm{pH}$. The $\mathrm{pH}$ varied over time due to residual $\mathrm{CaCO}_{3}$ dissolution, thus control samples collected over the settling period showed the metal removal as a function of $\mathrm{pH}$. The mixing regime was varied for the different HS dose orders, as shown in Figure 3-6. 


\section{Control}

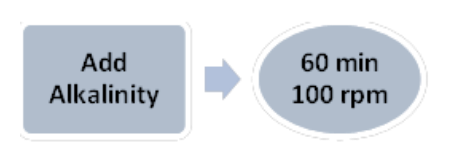

\section{Before}

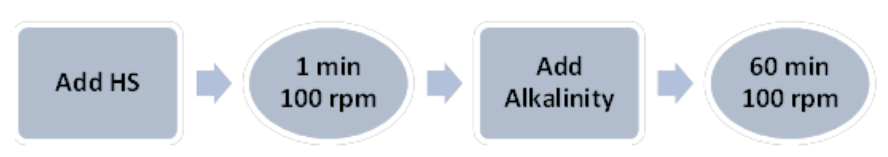

After

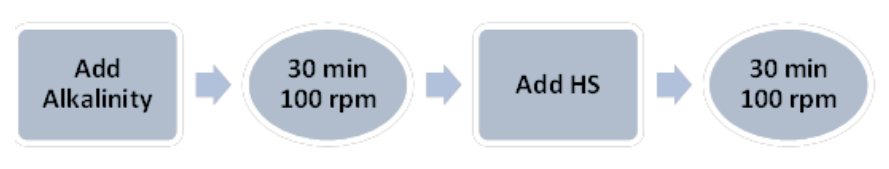

\section{During}

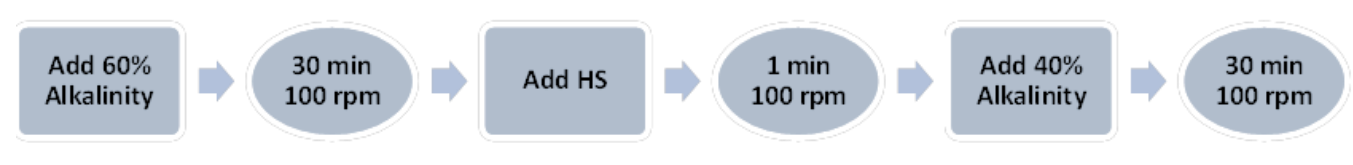

Figure 3-6: Mixing regime for HS dosed MSS scenario

Again, at the end of the mixing period the jars were removed from the jar stir apparatus and allowed to settle. There was no flocculation period in the MSS jar testing scenario to represent discharge of the turbulent UMS directly into the Mangatini Sump.

\subsubsection{MSS Polymer Mixing Conditions}

The polymer jar testing produced raw samples for measurement of water quality parameters, to investigate the potential application of HS as a polymer for removal of residual $\mathrm{CaCO}_{3} \mathrm{SS}$. These results are discussed in Chapter 6 (HS - Water Quality). Figure 3-7 shows how the polymer jar testing fits into the overall experimental plan with samples used for water quality analysis only.

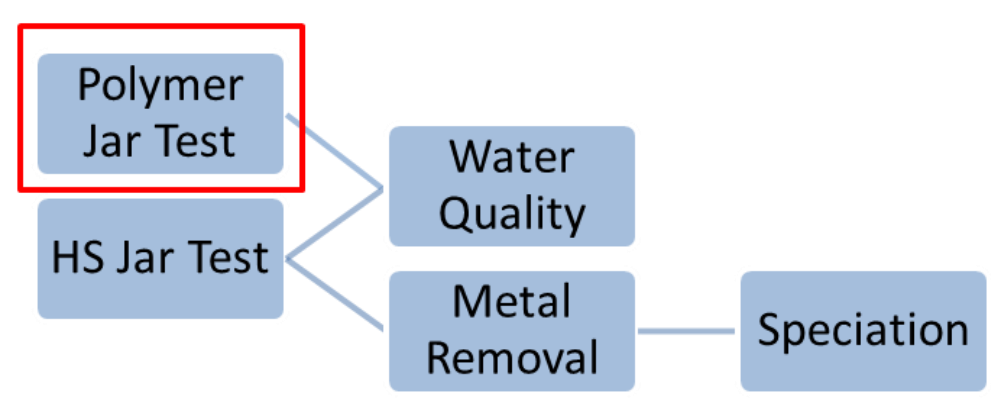

Figure 3-7: Polymer jar test context 
Methodology

The dose conditions for the $\mathrm{MSS} \mathrm{CaCO}_{3} \mathrm{SS}$ removal application were set to find the most efficient SEHA dose as a polymer. The performance of SEHA was evaluated against two commercially available polymers; Crystalfloc B570 and B610. Figure 3-8 shows the SEHA and polymer dose conditions of the polymer trials.

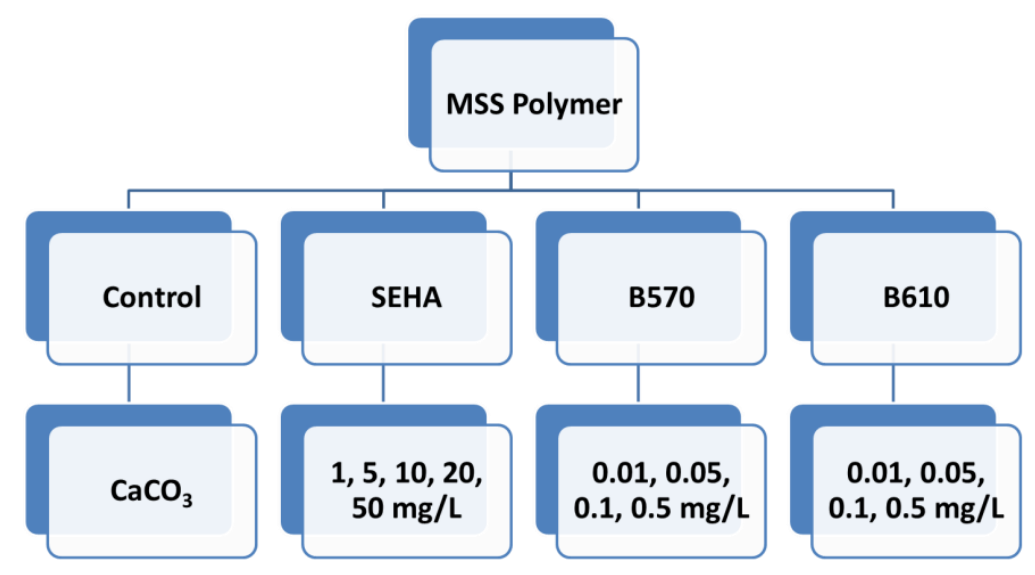

Figure 3-8: Dose conditions for polymer investigation

For control samples, one hour rapid mixing at $100 \mathrm{rpm}$ represented the residence time and turbulent flow of the stream. A sufficient $\mathrm{CaCO}_{3}$ dose was used to raise the $\mathrm{pH}$ of AMD to 6 after one hour mixing. All SEHA and Crystalfloc dosed batches were neutralised by $\mathrm{CaCO}_{3}$ to $\mathrm{pH} 6$ and mixed for 55 minutes at $100 \mathrm{rpm}$. The HS/polymers were then added and stirred for a further five minutes at $100 \mathrm{rpm}$. These mixing conditions represent dosing of SEHA or polymer just above the discharge point of the UMS into the Mangatini Sump, utilising natural turbulence to mix polymer. The benefit of earlier polymer dosing with the aim to enhance flocculation would be minimal due to the turbulent flow limiting floc aggregation. At the end of the mixing period the beakers were removed from the jar stir apparatus and allowed to settle. 
Methodology

\subsubsection{Supernatant Sampling and Analysis}

This section describes supernatant sampling during the sedimentation period and analysis undertaken. All initial HS dosing and mixing conditions were described in section 3.3.3.

\subsubsection{Water Quality}

Supernatant samples were taken from both HS and polymer jar tests to measure effect of HS addition on water quality parameters during neutralisation of AMD. Figure 3-9 shows how samples collected from both the HS and polymer jar test supernatants were used in the water quality analysis.

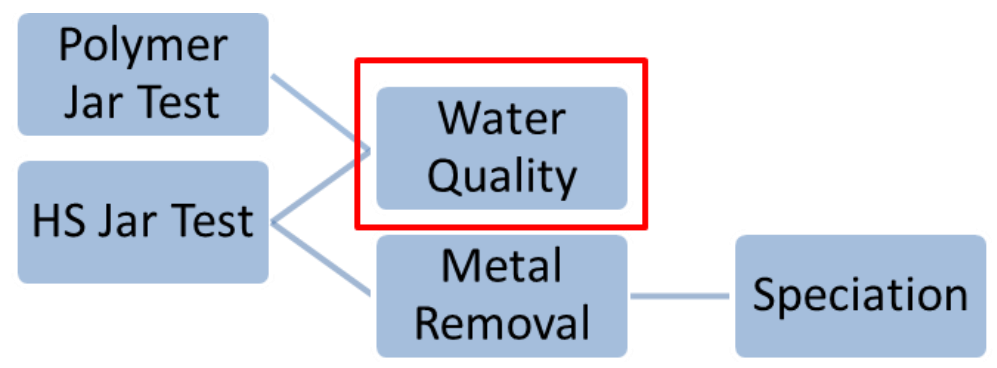

Figure 3-9: Water quality measurement context

\subsection{BTP Water Quality}

No water quality samples for the BTP trials were collected during the initial sedimentation period (0-10 min). This was because sampling at two minute intervals for metal analysis during the first 10 minutes was too variable. Therefore, supernatant samples for turbidity analysis were collected 30, 60 and 120 minutes into the sedimentation period, which are more representative of treatment plant residence times. A $10 \mathrm{~mL}$ sample was collected using a syringe from approximately $10 \mathrm{~mm}$ below the water surface to minimise disturbance of settling floc.

After 120 min sedimentation the $\mathrm{pH}$ was measured and the final turbidity sample was taken. Then $500 \mathrm{~mL}$ of supernatant was decanted into a measuring cylinder for SS analysis. These sampling regimes were repeated for both $\mathrm{NaOH}$ and $\mathrm{Ca}(\mathrm{OH})_{2}$ trials.

\subsection{MSS Water Quality}

A total of 12,1 L batches of AMD were neutralised for each $\mathrm{CaCO}_{3}$ condition. Therefore, six duplicated samples were collected at 2, $6 \mathrm{hrs}, 1,3,5$, and 7 days into the sedimentation period. For each sedimentation period a $10 \mathrm{~mL}$ sample was extracted for turbidity measurement as well as approximately $40 \mathrm{~mL}$ for $\mathrm{pH}$ measurement. Finally, 500 $\mathrm{mL}$ of supernatant was decanted for SS measurement. 
Methodology

\subsection{MSS Polymer Water Quality}

Six supernatant samples were taken $0.5,1,2,3,6$ and 24 hours into the sedimentation period for the polymer investigation. A $1 \mathrm{~L}$ batch of AMD was treated for each duplicated sedimentation period (12 total), so all samples were analysed for turbidity, $\mathrm{SS}$, and $\mathrm{pH}$.

\subsubsection{Metal Removal}

Samples were also collected from HS jar tests for measurement of supernatant metal concentration, as shown in Figure 3-10.

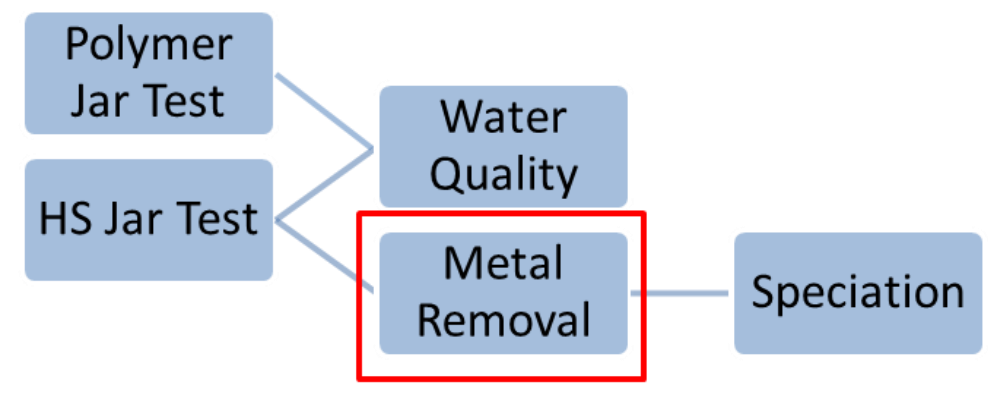

Figure 3-10: Metal removal measurement context

\subsection{BTP Metal Removal}

One supernatant sample was taken for each of the twelve control suite batches, at increments between $\mathrm{pH} 6$ and 7. Therefore, twelve samples were taken in total for both the $\mathrm{NaOH}$ and $\mathrm{Ca}(\mathrm{OH})_{2}$ control suite. The samples were collected after one hour sedimentation (representative of the average metal concentration). A $10 \mathrm{~mL}$ sample was drawn off by syringe and filtered $(0.45 \mu \mathrm{m})$ into an ICP-MS tube. The sample was then acidified $(\mathrm{pH}<2)$ and refrigerated $\left(4{ }^{\circ} \mathrm{C}\right)$ for later dilution and ICP-MS analysis.

Eight supernatant samples were taken in total for HS jar tests at 2, 4, 6, 8, 10, 30, 60, 120 minutes into the sedimentation period. All eight samples were taken from the same $1 \mathrm{~L}$ batch of neutralised AMD to show changes in metal concentration with respect to time. Samples were collected and stored as described for the control suite.

\subsection{MSS Metal Removal}

Six supernatant samples were taken $2,6 \mathrm{hrs}, 1,3,5$, and 7 days into the sedimentation period. A $10 \mathrm{~mL}$ supernatant sample was drawn off using a syringe and filtered into an ICP-MS tube. The sample was then acidified and stored for later dilution and ICP-MS analysis. 
Methodology

\subsubsection{Speciation Modelling}

Speciation modelling used the equilibrium supernatant metal concentrations determined by the metal removal analysis. Figure 3-11 shows how speciation modelling fits into the overall experiment plan.

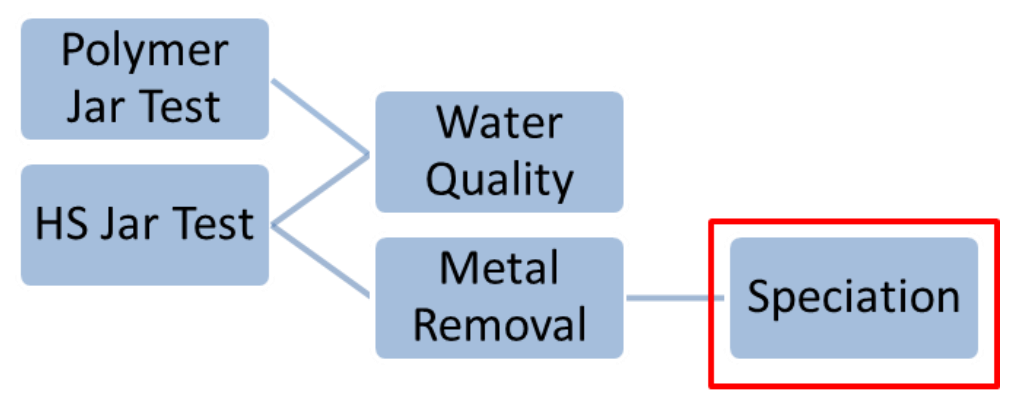

Figure 3-11: Speciation context

The equilibrium speciation model used was Visual MINTEQ, version 3.0 (Gustafsson 2010). Visual MINTEQ uses mass and charge balance equations, along with stability constants to determine the equilibrium speciation of a water sample. Major cation and anion species were determined by ICP-MS, resulting in an approximately neutral charge balance. The NICA-Donnan model was used to estimate HS-metal complexation to carboxylic and phenolic functional groups. The default metal-HS stability constants were used, as derived by Milne et al. (2003). The modelling results were exported for each cation/anion as a species distribution.

The metal concentrations used in the speciation modelling were obtained from ICP-MS analysis of filtered samples. The residual HS in the filtered samples was measured by UV-vis, and found to be very low. Therefore, two sets of speciation modelling were undertaken. The first set used HS concentrations determined from experimental samples. The second set used theoretical HS concentrations, representative of the range of HS measured in West Coast streams.

\subsubsection{Experimental Speciation}

For the experimental modelling, the HS concentration was determined by UV-vis spectrophotometry. The HS concentration after filtration of all HS conditions was measured and the sample with the greatest HS concentration for each alkalinity reagent $\left(\mathrm{NaOH}, \mathrm{Ca}(\mathrm{OH})_{2}\right.$ and $\left.\mathrm{CaCO}_{3}\right)$ was identified. The metal speciation was then determined using the HS and metal concentration determined by ICP-MS as input values. 
Methodology

\subsubsection{Theoretical Speciation}

The theoretical speciation modelling used the ICP-MS measured metal concentration of a control sample at approximately $\mathrm{pH} 6.6$ for each alkalinity reagent. The model was then run using a range of $\mathrm{HS}$ concentrations from 0.5 to $100 \mathrm{mg} / \mathrm{L}$, which represent the broad range of natural HS concentrations observed in West Coast streams (Collier and Winterbourn 1987; Collier 1989). The species distribution for each HS concentration was presented as figures showing metal speciation as a function of HS concentration. 


\section{Chapter 4. Material Characterisation}

The material characterisation chapter is divided into two sections.

Section 1: HS characterisation. This section discusses the results of humic substance (HS) characterisation, particularly identifying the abundance of reactive functional groups important in metal complexation.

Section 2: Sludge characterisation. This section discusses the composition of acid mine drainage (AMD) sludge.

\subsection{Humic Substances}

The elemental composition of W7 lignite (parent material of Solid Energy Humic Acid (SEHA)), SEHA and Aldrich Humic Acid (AHA) are shown in Table 4-1. The characterisation of W7 lignite was included to show the changes in HS structure that occur during alkali extraction to form SEHA.

Table 4-1: Elemental mass composition of HS as a percentage

\begin{tabular}{cccccc}
\hline & W7 & LEHA & AHA & HA & Fange* \\
\hline $\mathbf{C}$ & 57.7 & 40 & 37.2 & $50-60$ & $40-50$ \\
$\mathbf{H}$ & 5.14 & 4.05 & 3.29 & $3-6$ & $4-7$ \\
$\mathbf{O}$ & 31.1 & 40 & 25.8 & $30-38$ & $40-50$ \\
$\mathbf{N}$ & 0.73 & 0.55 & 0.74 & $0.8-4.3$ & $0.9-3.3$ \\
$\mathbf{S}$ & $<0.3$ & $<0.3$ & $<0.3$ & $0.1-2$ & $0.1-3.6$ \\
$\mathbf{A s h}$ & 8.6 & 63.7 & 32.5 & & \\
$\mathbf{C / O}$ & & & & & \\
(** & 2.48 & 1.33 & 1.92 & - & \\
$\mathbf{C}^{* *}$ & 0.94 & 0.82 & 0.94 & - & \\
\hline
\end{tabular}

*(Schnitzer and Kahn 1972; Stevenson 1994)

** On a molar basis

The composition of the W7 lignite falls within the typical range of CHON for humic acid (HA) shown on Table 4-1 (Schnitzer and Hansen 1970; Stevenson 1994). The W7 lignite 
had a 71 wt\% HA content (determined by SENZ using ISO 5073), on a dry basis, which has been reflected in the lignite $\mathrm{CHON}$ analysis.

The CHON results show SEHA is more typical of a fulvic acid (FA) than an HA, particularly with respect to carbon. The alkali extraction method used to prepare SEHA produces both humic and fulvic acid, thus the properties of both would be superimposed on the SEHA product. Thus, significant amount of FA may have been present in the SEHA product. A small amount of humin may also be present as centrifugation of the highly viscous SEHA product may not remove all insoluble (humin) particulate.

The elemental composition of AHA should fall within the range of CHON values on Table 4-1, as AHA is a commercial HA product. The carbon concentration is very low, suggesting the AHA is dominated by FA. The oxygen concentration is also low, at $25 \%$, which is more typical of a heavy HA structure. Monteil-Rivera et al. (2000) performed $\mathrm{CHON}$ analysis on an AHA sample washed several times using acid solutions to remove inorganic solids and Fe. Their analysis of the acid washed AHA sample showed values of $\mathrm{C}, \mathrm{H}, \mathrm{O}$, and $\mathrm{N}$ within the ranges identified on Table 4-1. Thus, the purity of the AHA and SEHA stock solutions used in this thesis may have affected the CHON analysis, as these materials were not acid washed before analysis.

The high ash content reported for SEHA may have been partially due to Na complexed to functional groups after $\mathrm{NaOH}$ extraction from lignite. Extraction of $\mathrm{HS}$ from W7 lignite using a $0.5 \mathrm{M} \mathrm{NaOH}$ solution would result in a $\mathrm{Na}$ concentration in SEHA solution in the order of $11.5 \mathrm{~g} / \mathrm{L}$, or approximately $1.2 \%$. The HA concentration of the SEHA product was $6.69 \%$. Thus, when the SEHA was dried for analysis, the $1.2 \% \mathrm{Na}$ from SEHA solution was concentrated to around 15\% of the solid weight. The W7 lignite (parent material of SEHA) was $8.6 \mathrm{wt} \%$ ash, as shown on Table 4-1. Extraction of this inorganic material to make SEHA, and consequent concentration during the SEHA drying process may have significantly increased the ash content of SEHA. Further work is required to test the validity of these ash results for both SEHA and AHA.

The structure of HS was investigated using both FTIR and NMR. Figure 4-1 shows the qualitative composition of HS determined by FTIR. 


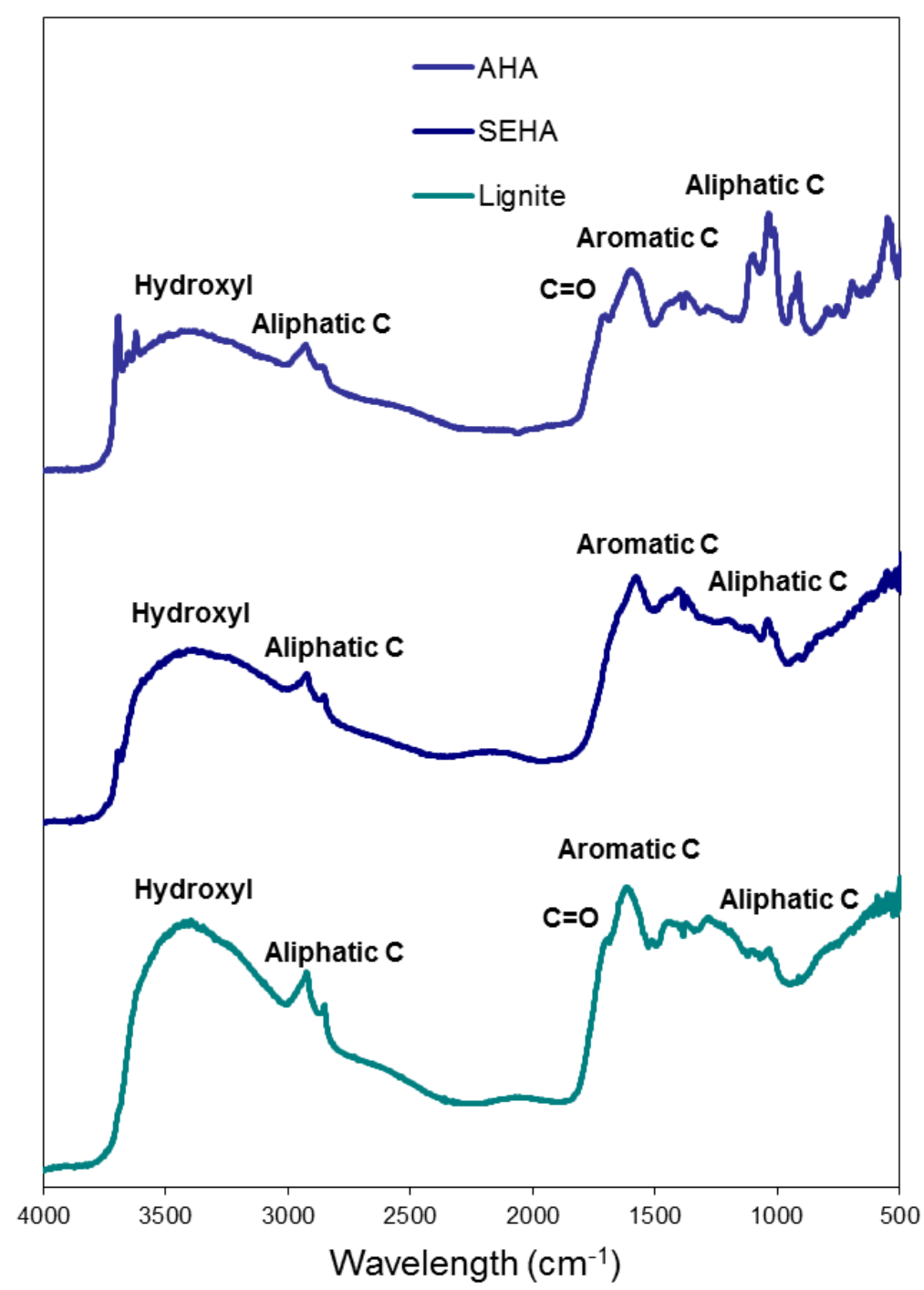

Figure 4-1: FTIR spectra of HS

The FTIR analysis identified aliphatic and aromatic carbon structures on all three HS (for peak assignments refer to section 3.2.1.2). Hydroxyl/phenolic groups appeared most significantly on the raw W7 lignite. Carboxylic groups were identified on both W7 lignite and AHA. The concentration of carboxylic and hydroxyl groups on SEHA was low compared to raw W7 lignite, indicating some loss of functional groups during the extraction processes.

A more quantitative analysis of HS composition was obtained by NMR. The NMR spectrum for the HS materials is shown on Figure 4-2. 


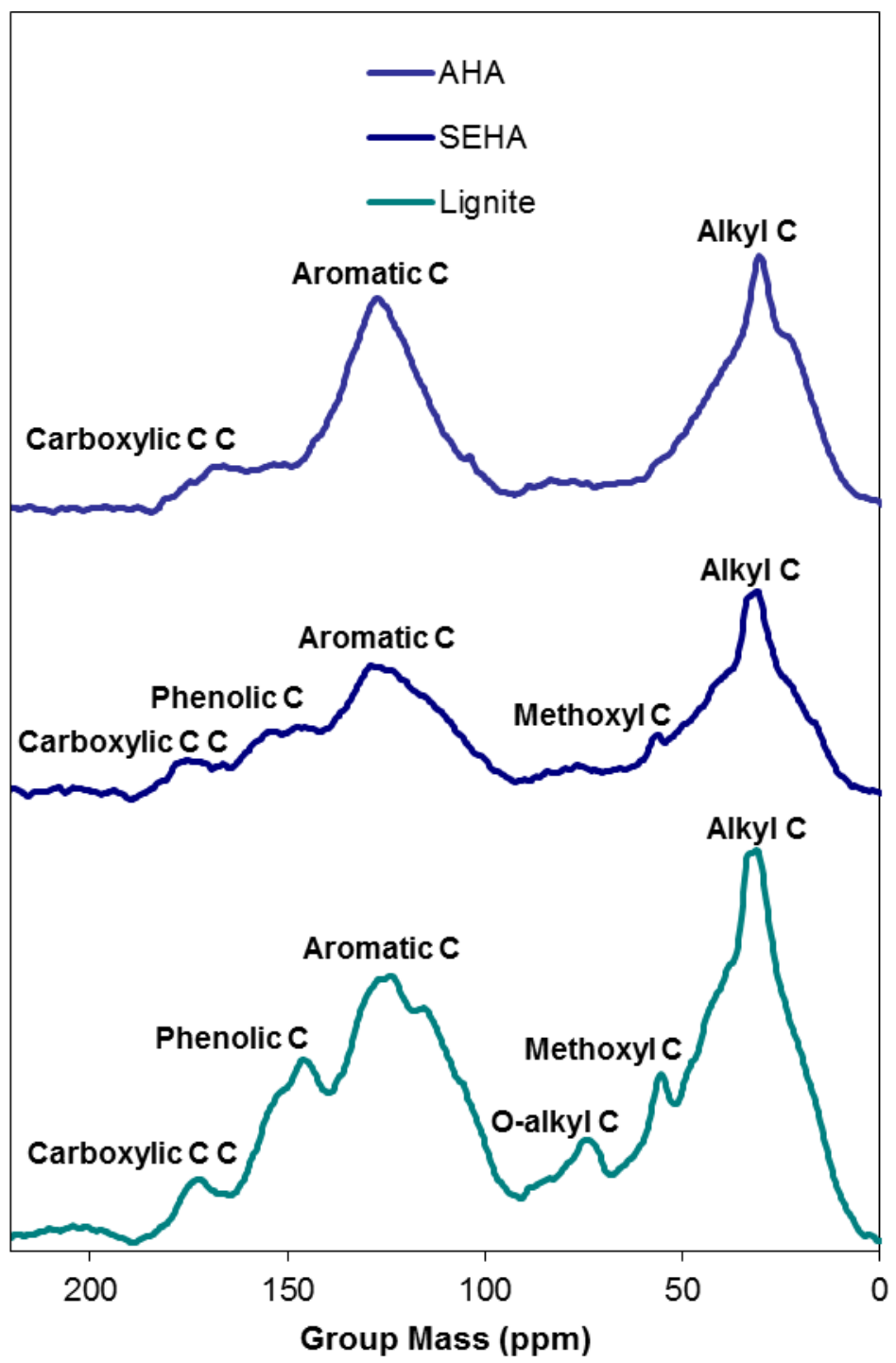

Figure 4-2: NMR spectra of HS

The NMR spectra show strong aromatic and aliphatic carbon peaks for all HS materials (for peak assignments refer to section 3.2.1.3). The carboxylic and phenolic peaks for SEHA were considerably less defined than the W7 lignite, which again suggests that reactive functional groups were lost during alkali extraction.

The quantitative distribution of HS components was determined by integration of the area under the peaks of the NMR spectra is shown on Table 4-2. 
Material Characterisation

Table 4-2: Distribution of carbon in HS structural groups

\begin{tabular}{lccccc}
\hline & W7 & LEHA & AHA & \multicolumn{2}{c}{ Range } \\
& Lignite & HA & FA $^{*}$ \\
\hline Aliphatic (0-50ppm) & 33.8 & 35.2 & 39.2 & $17-30$ & 22 \\
Carbohydrate (50-100ppm) & 17 & 14 & 11.3 & $12-18$ & 20 \\
Aromatic (100-140ppm) & 30.2 & 29.8 & 24.6 & $24-42$ & 26 \\
Phenolic (140-165ppm) & 12.1 & 11.9 & 8.7 & $5.9-9.5^{* *}$ & \\
Carboxylic (165-190ppm) & 4.9 & 6.8 & 5.3 & $12-18$ & 24 \\
\hline & (Tipping 2002) & & \\
&
\end{tabular}

All three HS were prepared by acid washing for NMR analysis. The structural group distribution of HS measured by NMR was closer to the typical ranges found in the literature.

The concentration of SEHA oxygen-containing functional groups was relatively low compared to the typical range for HA and FA. The SEHA had a high aliphatic and aromatic carbon concentration, suggesting a lower density of reactive oxygen-containing functional groups. Phenolic functional groups were more common than carboxylic groups on SEHA.

The AHA sample had a lower functional group concentration than SEHA, which suggests that SEHA would have a greater metal complexation capacity. The distribution of aliphatic and carboxylic groups on SEHA and AHA were outside the expected range for HA quoted from Tipping (2002). Monteil-Rivera (2000) also analysed AHA by NMR and showed most AHA groups fitted within the range described by Tipping (2002). Further analysis is required to resolve this discrepancy.

The extraction of HS from raw lignite results in a decrease in oxygen functional group concentration and an increase in the carbon content (Weber, Tang et al. 2006). Table 4-2 shows a small increase in aliphatic and carboxylic carbon through SEHA extraction from W7 lignite with a slight decrease in aromatic, phenolic and carbohydrate carbon. The loss of functional groups appears less significant when determined by NMR (quantitative analysis) compared to FTIR (qualitative analysis). 


\subsection{AMD Sludge}

Analysis of lab $\left(\mathrm{CaCO}_{3}\right.$ and $\mathrm{Ca}(\mathrm{OH})_{2}$ neutralised $)$ and field $\left(\mathrm{Ca}(\mathrm{OH})_{2}\right)$ AMD sludge by FTIR qualitatively identified the $\mathrm{FeOOH}$ and $\mathrm{CaCO}_{3}$ groups on Figure 4-3. NB: Field sludge was collected from the Blackwater Treatment Plant (BTP) and used in other projects; field XRF results are used here to show mineralogy of $\mathrm{Ca}(\mathrm{OH})_{2}$ neutralised AMD sludge.

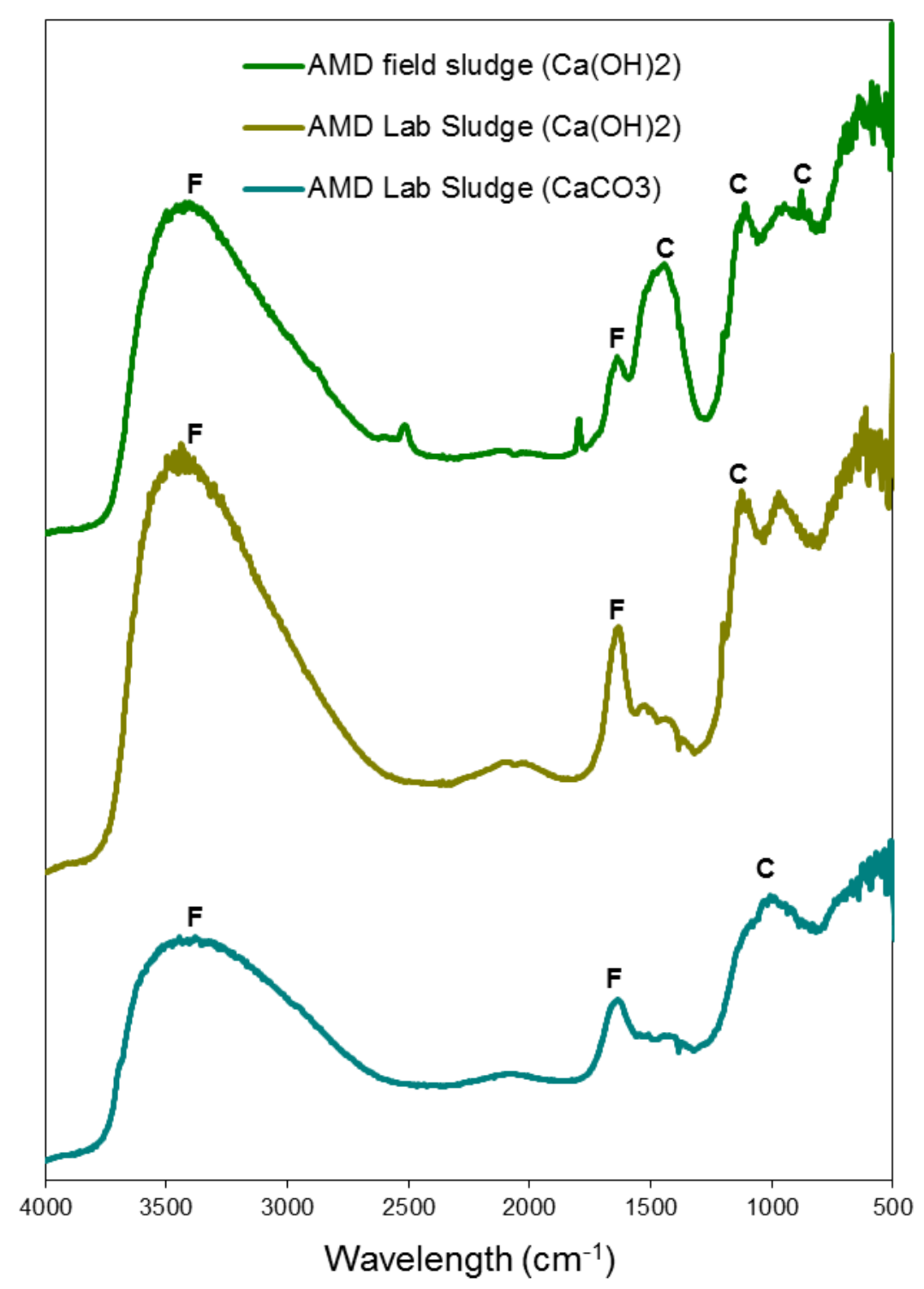

Figure 4-3: FTIR spectra of AMD sludge

NB: $\mathrm{F}$ and $\mathrm{C}$ correspond to $\mathrm{FeOOH}$ and $\mathrm{CaCO}_{3}$ absorbance peaks.

Peaks for $\mathrm{FeOOH}$ and $\mathrm{CaCO}_{3}$ were identified for all AMD sludge samples (for peak assignments refer to section 3.2.2.1). Greater detection of $\mathrm{CaCO}_{3}$ peaks was expected for the $\mathrm{CaCO}_{3}$ neutralised sludge due to the low dissolution efficiency of $\mathrm{CaCO}_{3}$. 
The elemental composition of crystalline mineral groups present in $\mathrm{Ca}(\mathrm{OH})_{2}$ (field) and $\mathrm{CaCO}_{3}$ (lab) neutralised AMD sludge were quantitatively determined using XRF, as shown in Figure 4-4.

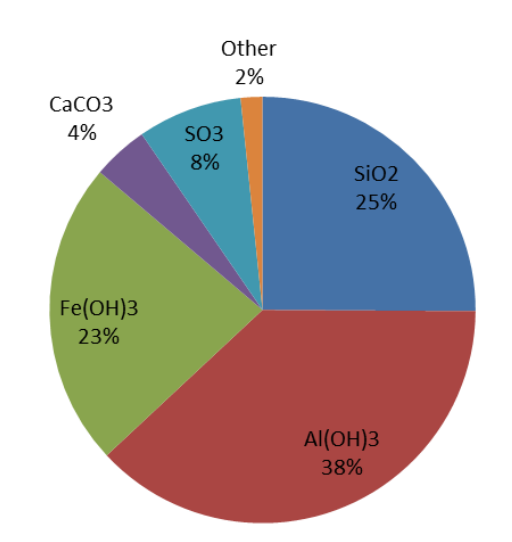

$\mathrm{Ca}(\mathrm{OH})_{2}$ AMD sludge (Field)

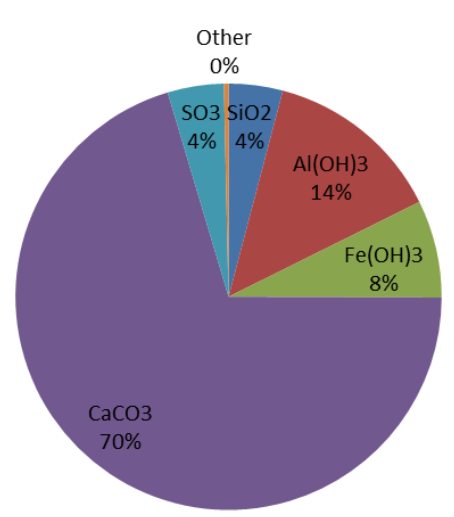

$\mathrm{CaCO}_{3} \mathrm{AMD}$ sludge (Lab)

Figure 4-4: XRF composition of AMD sludge

NB: XRF analysis determines the elemental composition of the AMD sludge. The detected $\mathrm{Fe}$, $\mathrm{Al}$, and $\mathrm{Ca}$ were assumed to correspond to $\mathrm{Fe}(\mathrm{OH})_{3}, \mathrm{Al}(\mathrm{OH})_{3}$ and $\mathrm{CaCO}_{3}$ species only, while other metals were assumed to correspond to oxides. The composition of sludge has been adjusted to reflect the greater molecular weight of the metal oxide/hydroxides/carbonates. The mineralogy on Figure 4-4 may not fully represent the actual sludge mineralogy.

Figure 4-4 clearly shows the $\mathrm{Ca}(\mathrm{OH})_{2}$ neutralised AMD sludge is rich in $\mathrm{Fe}(\mathrm{OH})_{3}$ and $\mathrm{Al}(\mathrm{OH})_{3}$, at 23 and $38 \mathrm{wt} \%$, respectively. The $\mathrm{CaCO}_{3}$ neutralised AMD sludge is dominated $(70 \mathrm{wt} \%)$ by $\mathrm{CaCO}_{3}$, due to only $50 \%$ dissolution of $\mathrm{CaCO}_{3}$, which is discussed further in section 5.2. The decrease in both $\mathrm{Fe}(\mathrm{OH})_{3}$ and $\mathrm{Al}(\mathrm{OH})_{3}$ concentration (compared to $\mathrm{Ca}(\mathrm{OH})_{2}$ neutralised sludge) is approximately $70 \mathrm{wt} \%$, suggesting the total mass of $\mathrm{Al}$ and $\mathrm{Fe}$ hydroxides was conserved, but their concentration diluted by residual $\mathrm{CaCO}_{3}$. The high $\mathrm{SiO}_{2}$ shown for the field sludge may be due to higher proportion $\mathrm{Si}$ containing suspended sediments present in the field samples.

The results of XRD support the findings of XRF. The $\mathrm{Ca}(\mathrm{OH})_{2}$ neutralised field sludge had high $\mathrm{Al}$ and $\mathrm{Fe}$ concentrations of 45 and $33 \mathrm{wt} \%$, however the speciation of these metals was not defined. The $\mathrm{Al}$ and $\mathrm{Fe}$ concentrations of $\mathrm{CaCO}_{3}$ neutralised AMD sludge were 6 and $5 \mathrm{wt} \%$, respectively and their speciation was also undefined; while $\mathrm{CaCO}_{3}$ made up 89 wt $\%$ of the sludge weight. Table 4-3 shows the mineralogy of AMD sludge 
Material Characterisation

determined by XRD, assuming all the Fe and $\mathrm{Al}$ exist as hydroxides. However, as the speciation of measured $\mathrm{Al}$ and $\mathrm{Fe}$ was not defined these results may not fully represent the actual sludge mineralogy.

Table 4-3: Mineralogy of AMD sludge

\begin{tabular}{ccc}
\hline & $\begin{array}{c}\mathrm{Ca}(\mathrm{OH})_{2} \text { AMD sludge } \\
\text { (field) }\end{array}$ & $\begin{array}{c}\mathrm{CaCO}_{3} \text { AMD sludge } \\
(\mathrm{Lab})\end{array}$ \\
\hline $\mathrm{Al}(\mathrm{OH})_{3}$ & 46.7 & 12.6 \\
$\mathrm{Fe}(\mathrm{OH})_{3}$ & 42.5 & 10.0 \\
$\mathrm{SiO}_{2}$ & 22 & - \\
$\mathrm{CaCO}_{3}$ & - & 77.4 \\
\hline
\end{tabular}

\subsection{Summary}

Characterisation of HS showed that both SEHA and AHA were relatively impure, due to high inorganic material concentrations from extraction, stock solution preparation or parent material. Thus, elemental and functional group compositions for SEHA and AHA were outside the typical ranges for HS obtained from the literature. A high proportion of phenolic functional groups were identified on SEHA which were shown to have the greatest metal complexation affinity (Milne, Kinniburgh et al. 2003); carboxylic and carbohydrate groups were also identified. The functional group concentration of SEHA was greater than AHA, suggesting SEHA would have a greater metal complexation capacity.

Characterisation of AMD sludge showed the formation of $\mathrm{Fe}$ and $\mathrm{Al}$ hydroxides at high concentrations from $\mathrm{NaOH}$ and $\mathrm{Ca}(\mathrm{OH})_{2}$ neutralised AMD. However, the mineralogy of $\mathrm{CaCO}_{3}$ neutralised AMD sludge was dominated by unreacted $\mathrm{CaCO}_{3}$, due to the low efficiency of $\mathrm{CaCO}_{3}$ neutralisation. Therefore $\mathrm{NaOH}$ and $\mathrm{Ca}(\mathrm{OH})_{2}$ AMD sludge had a greater metal adsorption capacity as the $\mathrm{CaCO}_{3}$ neutralised sludge has been diluted by unreacted $\mathrm{CaCO}_{3}$. 


\section{Chapter 5. Neutralisation Dose}

The neutralisation dose chapter is divided into two sections.

Section 1: BTP neutralisation dose. This chapter reports the dose of both $\mathrm{NaOH}$ and $\mathrm{Ca}(\mathrm{OH})_{2}$ required for neutralisation of acid mine drainage (AMD) to $\mathrm{pH} 7$.

Section 2: MSS neutralisation dose. This chapter reports the dose of $\mathrm{CaCO}_{3}$ required for neutralisation of AMD to $\mathrm{pH} 6$ and discusses the low neutralisation efficiency of $\mathrm{CaCO}_{3}$.

\subsection{BTP Neutralisation Dose}

Figure 5-1 shows the Blackwater Treatment Plant (BTP) neutralisation pathway for $\mathrm{NaOH}$ and $\mathrm{Ca}(\mathrm{OH})_{2}$ neutralised AMD. Raw data are included in Appendix 1.

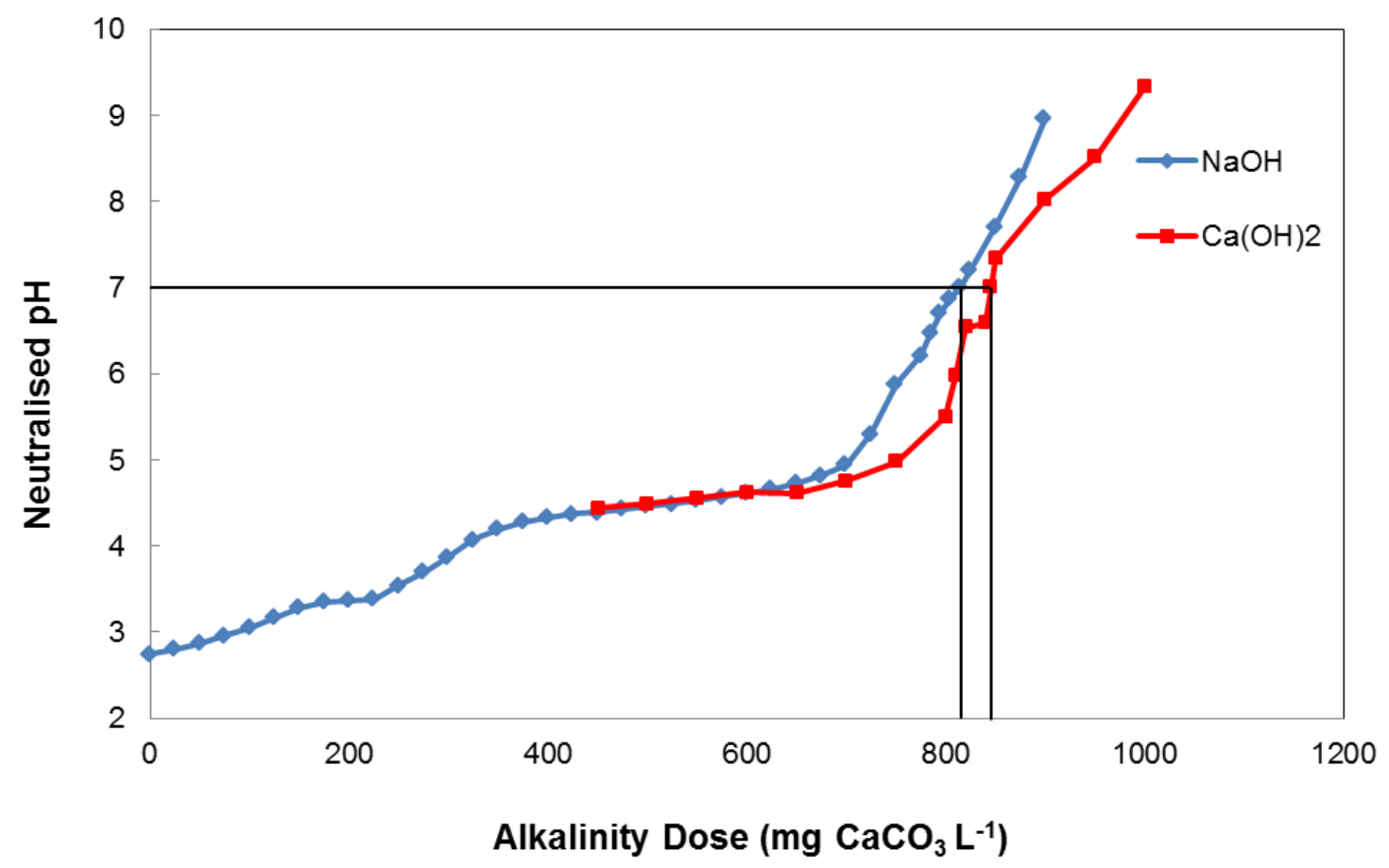

Figure 5-1: BTP neutralisation pathway

The neutralisation dose to $\mathrm{pH} 7$ for $\mathrm{NaOH}$ was $815 \mathrm{mg} / \mathrm{L} \mathrm{CaCO}_{3}$. This was achieved by adding $16.3 \mathrm{~mL}$ of $1 \mathrm{M} \mathrm{NaOH}$ solution. As $\mathrm{NaOH}$ dissolution was instantaneous and complete, $815 \mathrm{mg} / \mathrm{L} \mathrm{CaCO}_{3}$ is referred to generally as the required alkalinity dose for AMD neutralisation to $\mathrm{pH}$ 7. The neutralisation curve of $\mathrm{NaOH}$ shows two flat buffering sections where neutralisation is not proportional to $\mathrm{NaOH}$ dose. These buffering sections occur due to hydrolysis of precipitating metals, which act as buffers, increasing the total 
acidity. The first buffer occurs at $\mathrm{pH} 3.3$ and corresponds to the removal of Fe and the second buffer occurs at $\mathrm{pH} 4.5$ and corresponds to the removal of $\mathrm{Al}$ (Lee, Bigham et al. 2002).

The neutralisation dose to $\mathrm{pH} 7$ for $\mathrm{Ca}(\mathrm{OH})_{2}$ was $845 \mathrm{mg} / \mathrm{L} \mathrm{CaCO}$, achieved by adding $8.45 \mathrm{~mL}$ of $1 \mathrm{M} \mathrm{Ca}(\mathrm{OH})_{2}$ slurry. The $\mathrm{Ca}(\mathrm{OH})_{2}$ neutralisation curve overlays the $\mathrm{NaOH}$ neutralisation curve up to a $\mathrm{pH}$ of 4.7 , after which the $\mathrm{Ca}(\mathrm{OH})_{2}$ neutralisation becomes less efficient. Therefore, a greater neutralisation dose was required due to decreased dissociation and neutralisation efficiency at circum-neutral $\mathrm{pH}$. From Figure 5-1 the neutralisation efficiency of $\mathrm{Ca}(\mathrm{OH})_{2}$ at $\mathrm{pH} 7$ was $96 \%$, which is higher than the value of 90\% from the literature (Skousen, Sexton et al. 2000; Trumm 2010). The $\mathrm{Ca}(\mathrm{OH})_{2}$ used for was laboratory grade, over $99.5 \mathrm{wt} \%$ pure. As $621 \mathrm{mg}$ of $\mathrm{Ca}(\mathrm{OH})_{2}$ was added for neutralisation, approximately $30 \mathrm{mg}$ would have remained undissolved.

\subsection{MSS Neutralisation Dose}

Figure 5-2 shows the Mangatini Stream-sump System (MSS) neutralisation pathway for $\mathrm{CaCO}_{3}$ neutralised AMD. Raw data are included in Appendix 1.

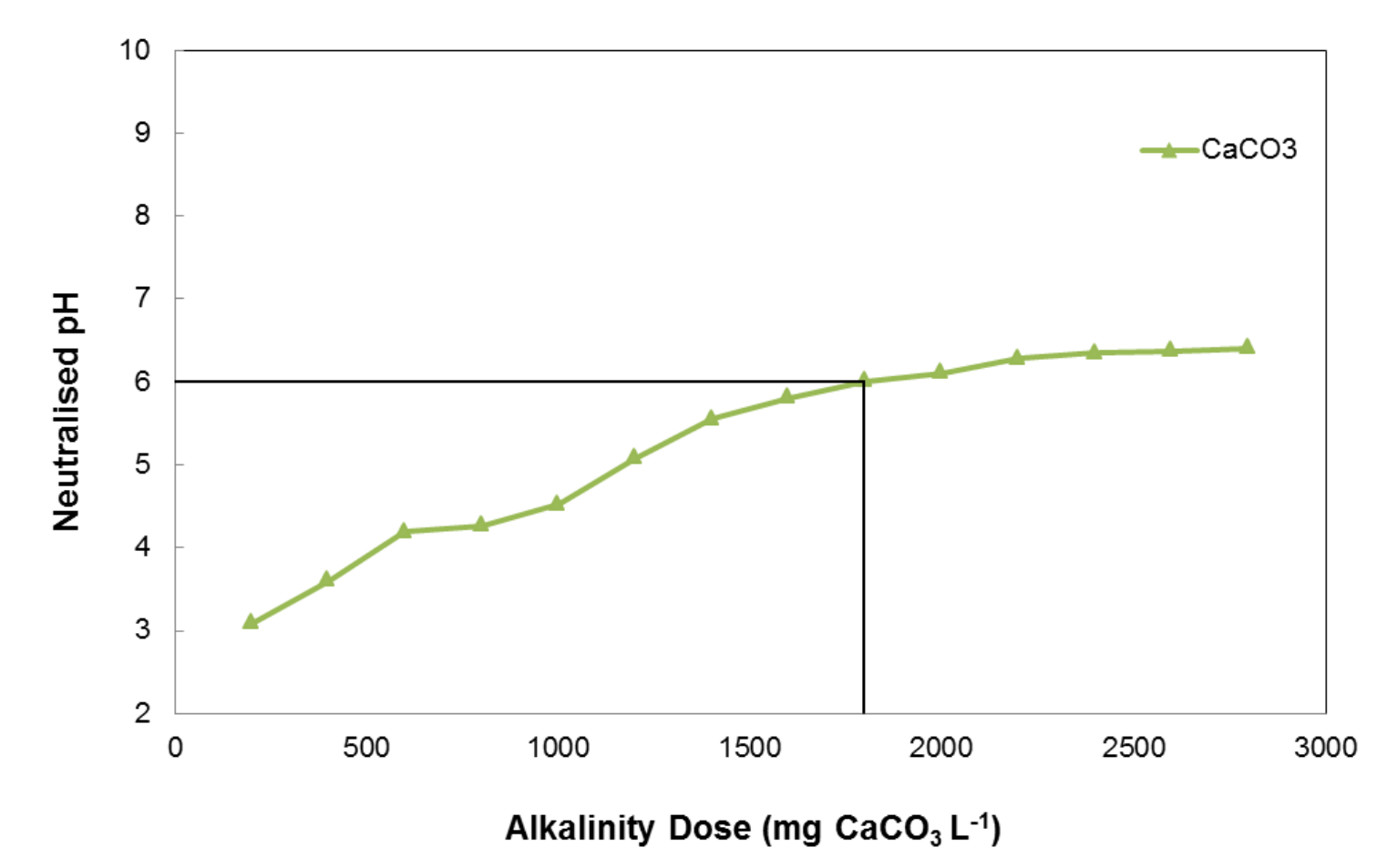

Figure 5-2: MSS neutralisation pathway

The neutralisation dose to $\mathrm{pH} 6$ (after one hour rapid mixing at $100 \mathrm{rpm}$ ) for $\mathrm{CaCO}_{3}$ was $1800 \mathrm{mg} / \mathrm{L} \mathrm{CaCO}_{3}$, suggesting a low $\mathrm{CaCO}_{3}$ neutralisation efficiency. This was achieved 
by adding $18 \mathrm{~mL}$ of $1 \mathrm{M} \mathrm{CaCO}_{3}$ slurry. The $\mathrm{CaCO}_{3}$ used in experimentation was determined to be $99 \mathrm{wt} \%$ pure by the EDTA titration method (APHA 2005), meaning $\mathrm{CaCO}_{3}$ impurity was not responsible for low neutralisation efficiency.

The $\mathrm{CaCO}_{3}$ neutralisation efficiency was lower than $\mathrm{NaOH}$ and $\mathrm{Ca}(\mathrm{OH})_{2}$ even at acidic $\mathrm{pH}$, suggesting $\mathrm{CaCO}_{3}$ has a low neutralisation efficiency. The $\mathrm{NaOH}$ neutralisation dose to $\mathrm{pH} 6$ was $760 \mathrm{mg} / \mathrm{L} \mathrm{CaCO}_{3}$. Thus, the neutralisation efficiency of $\mathrm{CaCO}_{3}$ to $\mathrm{pH} 6$ one hour after alkalinity dosing was $42 \%$, which is within the range of $30-70 \%$ cited from the literature (Skousen, Sexton et al. 2000; Trumm 2010).

After initial neutralisation to $\mathrm{pH} 6$ continued dissolution of $\mathrm{CaCO}_{3}$ over seven days resulted in a final $\mathrm{pH}$ of 8 , as shown on Figure 5-3.

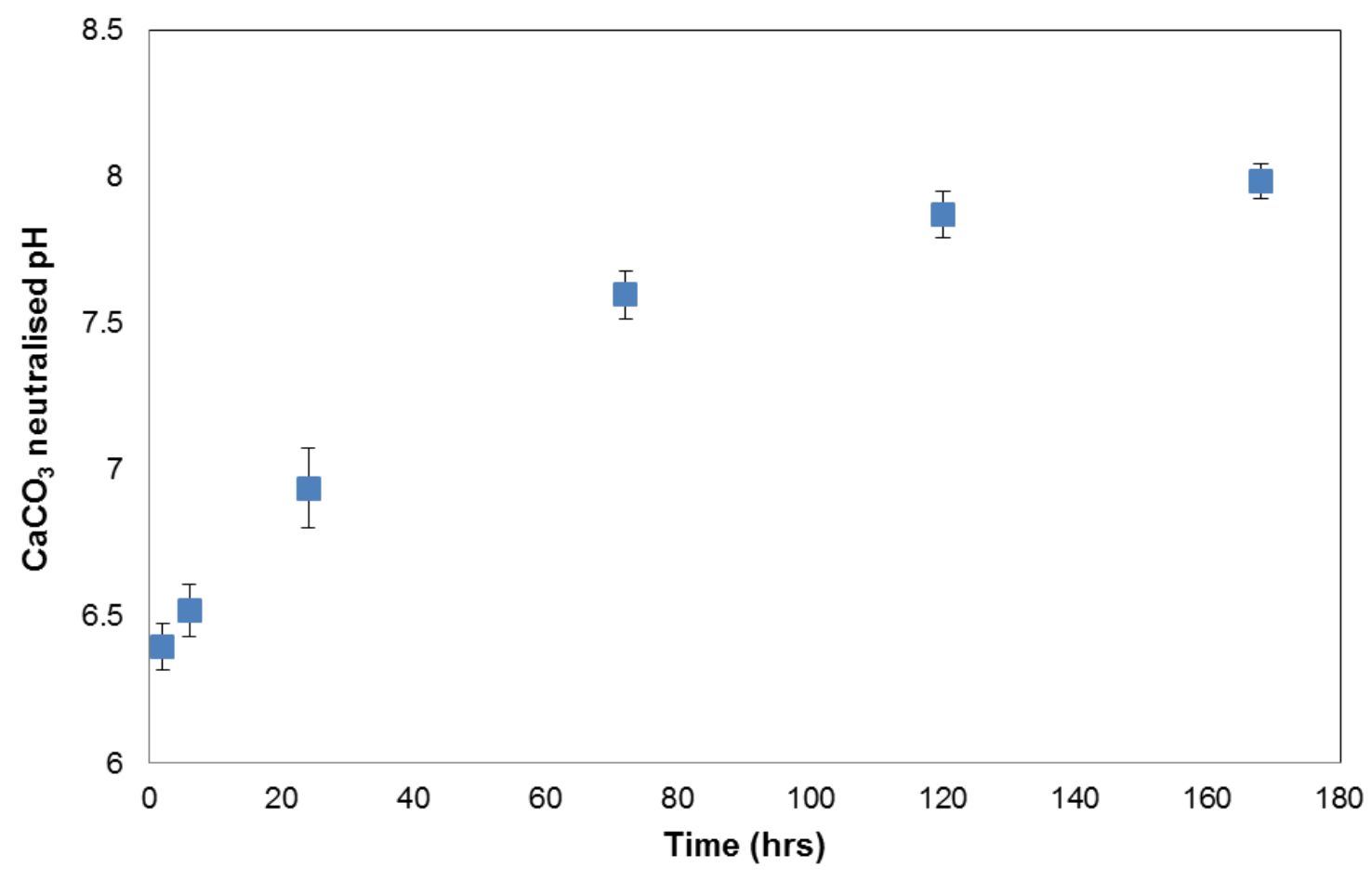

Figure 5-3: $\mathrm{CaCO}_{3}$ neutralised $\mathrm{pH}$ Standard deviation error $(\mathrm{n}=2)$.

The alkalinity dose by $\mathrm{NaOH}$ to $\mathrm{pH} 8$ was $860 \mathrm{mg} / \mathrm{L} \mathrm{CaCO}_{3}$. Therefore the neutralisation efficiency of $\mathrm{CaCO}_{3}$ increased to $48 \%$ for the original $1800 \mathrm{mg} / \mathrm{L} \mathrm{CaCO}_{3}$ dose after seven days. This shows that the rate of dissolution limits the neutralisation efficiency of $\mathrm{CaCO}_{3}$.

The engineering implications of neutralisation rates for $\mathrm{CaCO}_{3}$ are greater than $\mathrm{Ca}(\mathrm{OH})_{2}$ and $\mathrm{NaOH}$. Neutralisation by $\mathrm{Ca}(\mathrm{OH})_{2}$ and $\mathrm{NaOH}$ occurs relatively instantaneously, essentially eliminating dissolution kinetics from design consideration. Neutralisation by $\mathrm{CaCO}_{3}$ can be achieved more rapidly by greater alkalinity doses but residual unreacted 
$\mathrm{CaCO}_{3}$ may cause alkalinity issues in the receiving environment. Alternatively, the neutralisation efficiency of $\mathrm{CaCO}_{3}$ may be increased by long residence time applications allowing a greater dissolution of $\mathrm{CaCO}_{3}$ but requiring significantly larger tank/basin sizes. These factors have not been investigated in this thesis as the reaction period was defined by the Upper Mangatini Stream residence time of one hour.

Considering the neutralisation efficiencies discussed above, between 58 and $52 \%$ of $\mathrm{CaCO}_{3}$ remained unreacted in $\mathrm{CaCO}_{3}$ neutralised $\mathrm{AMD}$, corresponding to between 1044 and $936 \mathrm{mg}$ unreacted $\mathrm{CaCO}_{3}$ per litre at $\mathrm{pH} 6$ and 8, respectively. Unreacted limestone particles were visible within the sludge and the cloudy supernatant suggested suspended $\mathrm{CaCO}_{3}$ particulate was present.

\subsection{Summary}

The $\mathrm{NaOH}$ neutralisation dose to $\mathrm{pH} 7$ was $815 \mathrm{mg} / \mathrm{L} \mathrm{CaCO}_{3}$. As the neutralisation efficiency of $\mathrm{NaOH}$ is essentially $100 \%$, the $\mathrm{NaOH}$ neutralisation dose was also used as the required alkalinity dose for AMD neutralisation to $\mathrm{pH} 7$.

The $\mathrm{Ca}(\mathrm{OH})_{2}$ neutralisation dose to $\mathrm{pH} 7$ was slightly greater than $\mathrm{NaOH}$, at $845 \mathrm{mg} / \mathrm{L}$, due to a slightly lower neutralisation efficiency $(96 \%)$ at circum-neutral $\mathrm{pH}$. The decrease in neutralisation efficiency meant up to $30 \mathrm{mg} / \mathrm{L} \mathrm{Ca}(\mathrm{OH})_{2}$ would remain undissolved.

The $\mathrm{CaCO}_{3}$ neutralisation dose to $\mathrm{pH} 6$ of $1800 \mathrm{mg} / \mathrm{L} \mathrm{CaCO}_{3}$ was significantly greater than the $\mathrm{NaOH}$ equivalent $\left(760 \mathrm{mg} / \mathrm{L} \mathrm{CaCO}_{3}\right.$ to $\mathrm{pH}$ 6). Thus, the neutralisation efficiency of $\mathrm{CaCO}_{3}$ was $42 \%$. Continued dissolution of $\mathrm{CaCO}_{3}$ resulted in an increase to $\mathrm{pH} 8$ over the seven day sampling period, with a corresponding increase in neutralisation efficiency to $48 \%$. The low neutralisation efficiency meant that between 936 and $1044 \mathrm{mg} / \mathrm{L}$ of $\mathrm{CaCO}_{3}$ remained undissolved in the MSS trials. 


\section{Chapter 6. HS - Water Quality}

The HS - water quality chapter is divided into two sections.

Section 1: HS jar testing water quality. This section discusses the supernatant water quality for both the BTP and MSS application. The results of trials on the impact of humic substance (HS) dose order and concentration on turbidity and suspended solids (SS) for $\mathrm{NaOH}, \mathrm{Ca}(\mathrm{OH})_{2}$ and $\mathrm{CaCO}_{3}$ neutralised AMD are discussed. The HS dosed conditions are compared to controls to identify the impact of HS dosing on water quality.

Section 2: MSS polymer application. This section discusses the effectiveness of Solid Energy Humic Acid (SEHA) as a polymer for the removal of residual $\mathrm{CaCO}_{3} \mathrm{SS}$. The performance of SEHA is compared to two commercially available polymers. 
HS - Water Quality

\subsection{HS Jar Testing Water Quality}

This section discusses the water quality of the HS jar testing trails described in section 3.3.3.1. The water quality parameters investigated were turbidity and SS. The detection limits of turbidity and SS were considered to be $1 \mathrm{NTU}$ and $2 \mathrm{mg} / \mathrm{L}$ for the following discussion of results. The initial turbidity and SS of raw acid mine drainage (AMD) was negligible, due to settling during storage in the 1000 L IBC. The photographs in this section were taken 30 minutes into the sedimentation period. They have been included to illustrate qualitatively the difference in supernatant water quality, which is then quantitatively presented on the accompanying graphs.

\subsubsection{BTP Water Quality}

This section discusses the results of the Blackwater Treatment Plant (BTP) conditions from the $\mathrm{HS}$ jar testing experiments. The results from $\mathrm{NaOH}$ neutralisation are discussed first, followed by $\mathrm{Ca}(\mathrm{OH})_{2}$ results. The figures in this section show turbidity and SS for $\mathrm{NaOH}$ and $\mathrm{Ca}(\mathrm{OH})_{2}$ neutralised samples. Three turbidity samples were taken for each $\mathrm{HS}$ dose condition after 30, 60 and 120 minutes of sedimentation. One SS sample was taken at the end of the sedimentation period, at 120 minutes. All SS shown in these figures therefore correspond to SS after 120 minutes sedimentation. The control sample water quality results were determined from a representative $\mathrm{NaOH} / \mathrm{Ca}(\mathrm{OH})_{2}$ neutralised sample with $\mathrm{pH}$ 6.6. 
HS - Water Quality

\subsubsection{NaOH Water Quality}

Figure 6-1 shows the turbidity and SS of $\mathrm{NaOH}$ neutralised conditions. Raw data are included in Appendix 2.
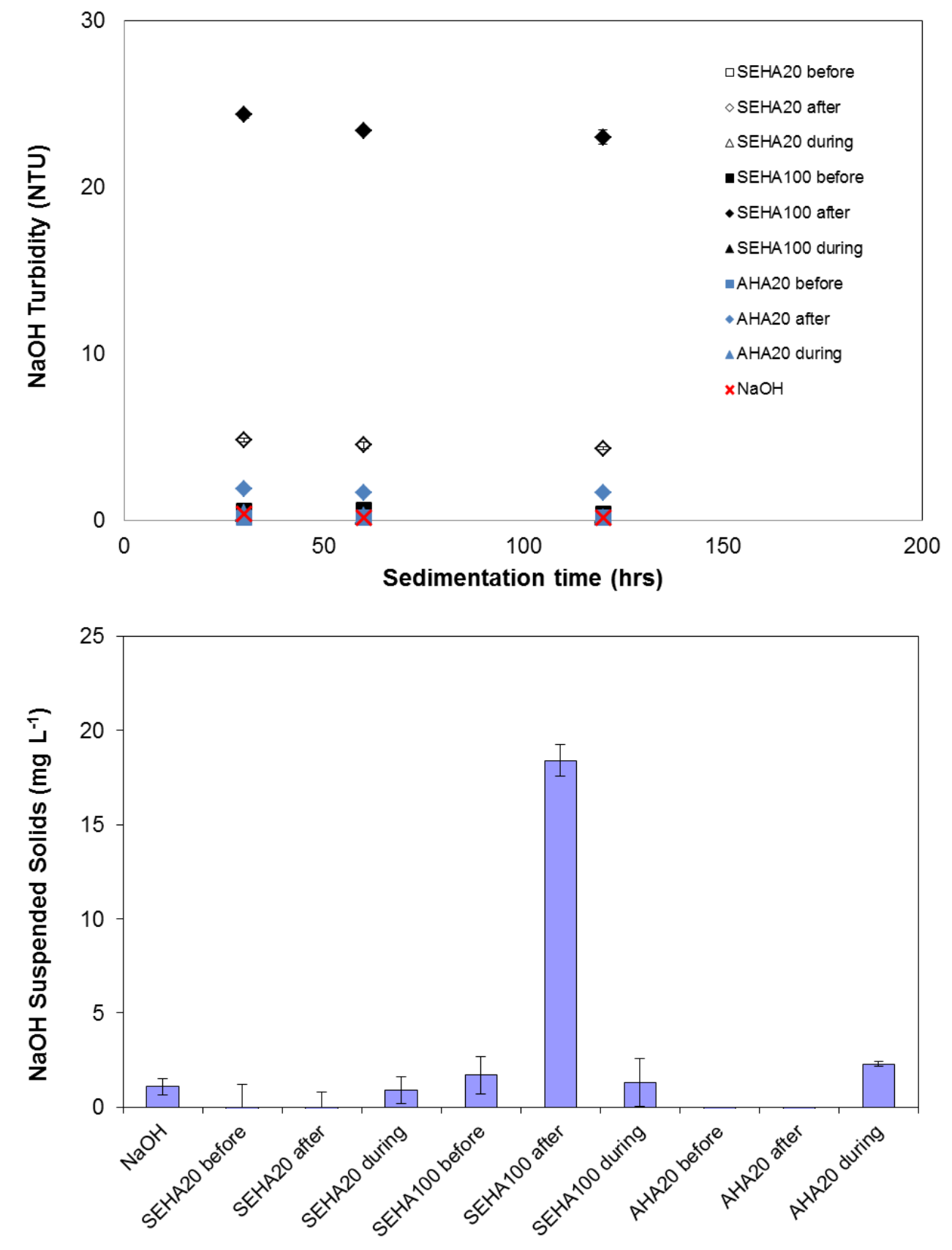

Figure 6-1: $\mathrm{NaOH}$ neutralised water quality Standard deviation error $(n=2)$.

The $\mathrm{NaOH}$ control neutralised samples had turbidity and SS below detection limits for both parameters. Neutralisation of the AMD formed insoluble metal hydroxide precipitates which naturally settled out of suspension. The dissociation of $\mathrm{NaOH}$ was 
HS - Water Quality

complete so the residual $\mathrm{NaOH}$ concentration was well below saturation limits and did not contribute to residual turbidity and SS.

The addition of SEHA and AHA before and during neutralisation of AMD resulted in turbidity of less than 2.0 NTU and SS below detection limits at all HS concentrations trialled. Figure 6-2 shows the clear and colourless supernatant of the control, before- and during-neutralisation conditions for $\mathrm{NaOH}$ neutralised AMD.

Addition of HS after neutralisation resulted in significant turbidity and SS. The SEHA100 after-neutralisation condition resulted in turbidity and SS of 24.4 NTU and $18.4 \mathrm{mg} / \mathrm{L}$, respectively. The SEHA20 and AHA20 after-neutralisation conditions also had high turbidity of 4.8 and 2.0 NTU respectively, while SS were below detection levels. The turbidity reduced with respect to time, but not rapidly enough for typical treatment plant residence times. The SEHA 100 after-neutralisation samples were at the maximum range of turbidity trigger values from 2-25 NTU suggested by ANZECC (2000). Figure 6-2 shows discolouration of the SEHA20 and SEHA100 after-neutralisation supernatants. 
HS - Water Quality

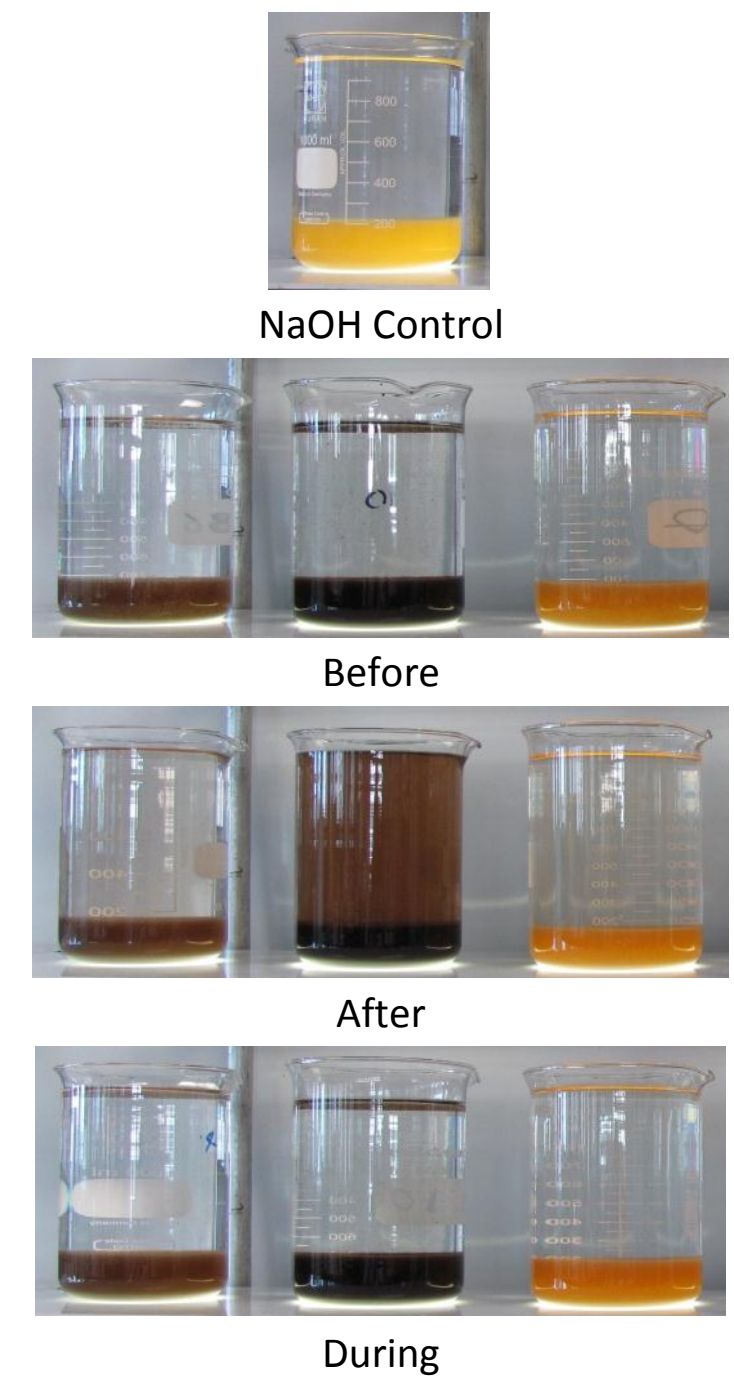

Figure 6-2: NaOH neutralised supernatant (L to R: SEHA20, SEHA100, AHA20)

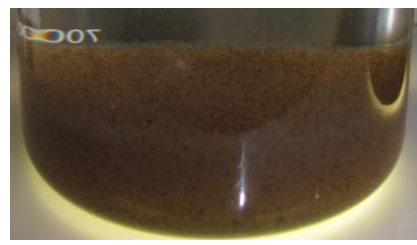

Before

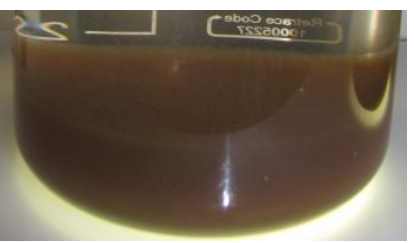

After

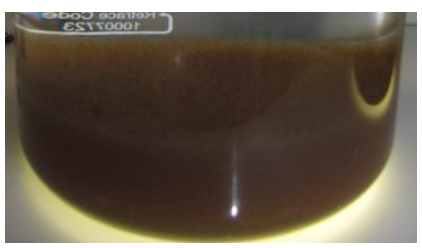

During

Figure 6-3: Comparison of $\mathrm{NaOH}$ neutralised SEHA20 sludge

Addition of HS before and during neutralisation resulted in clear and colourless supernatant (Figure 6-2). Figure 6-3 shows the sludge formed by HS addition at different stages of $\mathrm{NaOH}$ neutralisation. At $\mathrm{pH} 4.5$ and less, the solubility of $\mathrm{HS}$ in water is low, due to the insolubility of humic acid (HA) in acidic solution (Schnitzer and Kahn 1972). Addition of $\mathrm{HS}$ before and during neutralisation $(\mathrm{pH} \leq 4.5)$ therefore resulted in formation of HS precipitate. The HS precipitate was visible in the sludge during the 
HS - Water Quality

sedimentation period (after final neutralisation to $\mathrm{pH}$ 7), suggesting $\mathrm{HS}$ was not resolubilised at circum-neutral $\mathrm{pH}$.

The HS particles may have acted as a nucleation site for hydroxide precipitate aggregation, triggering flocculation. The dissociation constant of carboxylic groups on both HA and fulvic acid (FA) is around 3 (Milne, Kinniburgh et al. 2001). The PZC of $\mathrm{Al}(\mathrm{OH})_{3}$ and iron oxides range from 5.0 to 8.5 (Kretzschmar and Sticher 1997; Sparks 2003), thus at $\mathrm{pH}$ less than 5 all precipitated metal hydroxides would be positively charged. As $\mathrm{Fe}(\mathrm{OH})_{3}$ begins to precipitate (at $\mathrm{pH} 3.5$ ), a large portion of HS functional groups would be deprotonated and negatively charged, resulting in adsorption/coprecipitation of $\mathrm{Fe}(\mathrm{OH})_{3}$ onto the HS surface. When HS is added during neutralisation ( $\mathrm{pH} 4.5)$, the precipitation of $\mathrm{Al}(\mathrm{OH})_{3}$ (occurring simultaneously at $\mathrm{pH} 4.5$ ) may result in adsorption/co-precipitation of $\mathrm{Al}(\mathrm{OH})_{3}$ to $\mathrm{HS}$. Adsorption of hydroxide precipitates to HS would be exoected to result in particle aggregation and enhanced settleability.

Figure 6-3 shows that addition of HS after neutralisation results in a uniformly brown sludge, with no indication of precipitated HS. At $\mathrm{pH} 7$, carboxylic functional groups would be deprotonated and negatively charged, while some metal hydroxides, particularly $\mathrm{Al}(\mathrm{OH})_{3}$ would also be negatively charged, preventing HS adsorption. Thus, when added after neutralisation, there would be minimal incorporation of HS into the newly formed hydroxide flocs. Therefore, the elevated turbidity of 24.4 NTU for the SEHA100 afterneutralisation condition was probably caused by HS. This theory was supported by the measurement of a similar turbidity (21.8 NTU) for a $100 \mathrm{mg} / \mathrm{L}$ standard SEHA solution made up in circum-neutral deionised water.

Removal of HS by sweep-floc does not appear to occur when HS is added after neutralisation, probably due to electrostatic repulsion of HS from the hydroxide precipitates. This suggests the addition of deprotonated (anionic) HS to a neutralised AMD at equilibrium (i.e. $\mathrm{pH}$ 7) pushes the coagulation process back to zone three (Tchobanoglous, Burton et al. 2003), where residual turbidity is high.

Neutralisation by $\mathrm{NaOH}$ resulted in $\mathrm{Na}^{+}$concentrations of approximately $350 \mathrm{mg} / \mathrm{L}$, making $\mathrm{Na}^{+}$the dominant cation. Monovalent cations only bind to one HS reactive site. Therefore, $\mathrm{Na}^{+}$ions were unable to form an inter-particle bridge between HS molecules to form aggregate flocs. As the vast majority of $\mathrm{Al}$ and Fe were already precipitated well 
HS - Water Quality

below $\mathrm{pH} 7$, the coagulation and flocculation of HS added after neutralisation was poor, resulting in elevated residual turbidity and SS.

\subsubsection{2 $\mathrm{Ca}(\mathrm{OH})_{2}$ Water Quality}

Figure 6-4 shows the turbidity and SS of $\mathrm{Ca}(\mathrm{OH})_{2}$ neutralised conditions. Raw data are included in Appendix 2.
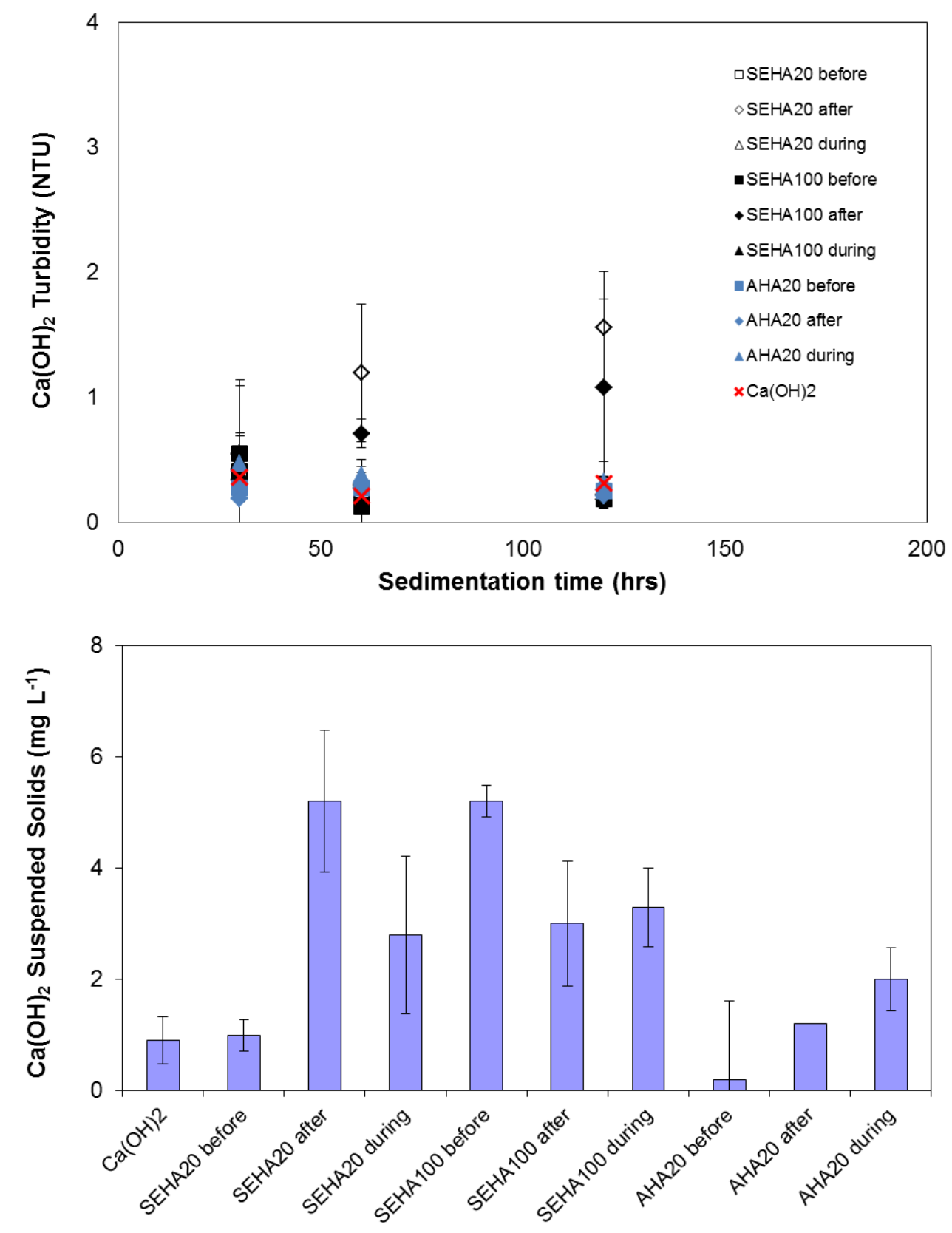

Figure 6-4: $\mathrm{Ca}(\mathrm{OH})_{2}$ neutralised water quality. Standard deviation error (n=2).

The $\mathrm{Ca}(\mathrm{OH})_{2}$ neutralised control resulted in turbidity and SS below detection levels. All HS dosed, $\mathrm{Ca}(\mathrm{OH})_{2}$ neutralised samples had turbidity and SS of less than 1.6 NTU and 
HS - Water Quality

$5.2 \mathrm{mg} / \mathrm{L}$, respectively. There were no conditions with turbidity or SS as elevated as the $\mathrm{NaOH}$ neutralised SEHA100 after-neutralisation condition (discussed in section 6.2.1.1). Figure 6-5 shows the relatively clear and colourless supernatants of $\mathrm{Ca}(\mathrm{OH})_{2}$ neutralised AMD.

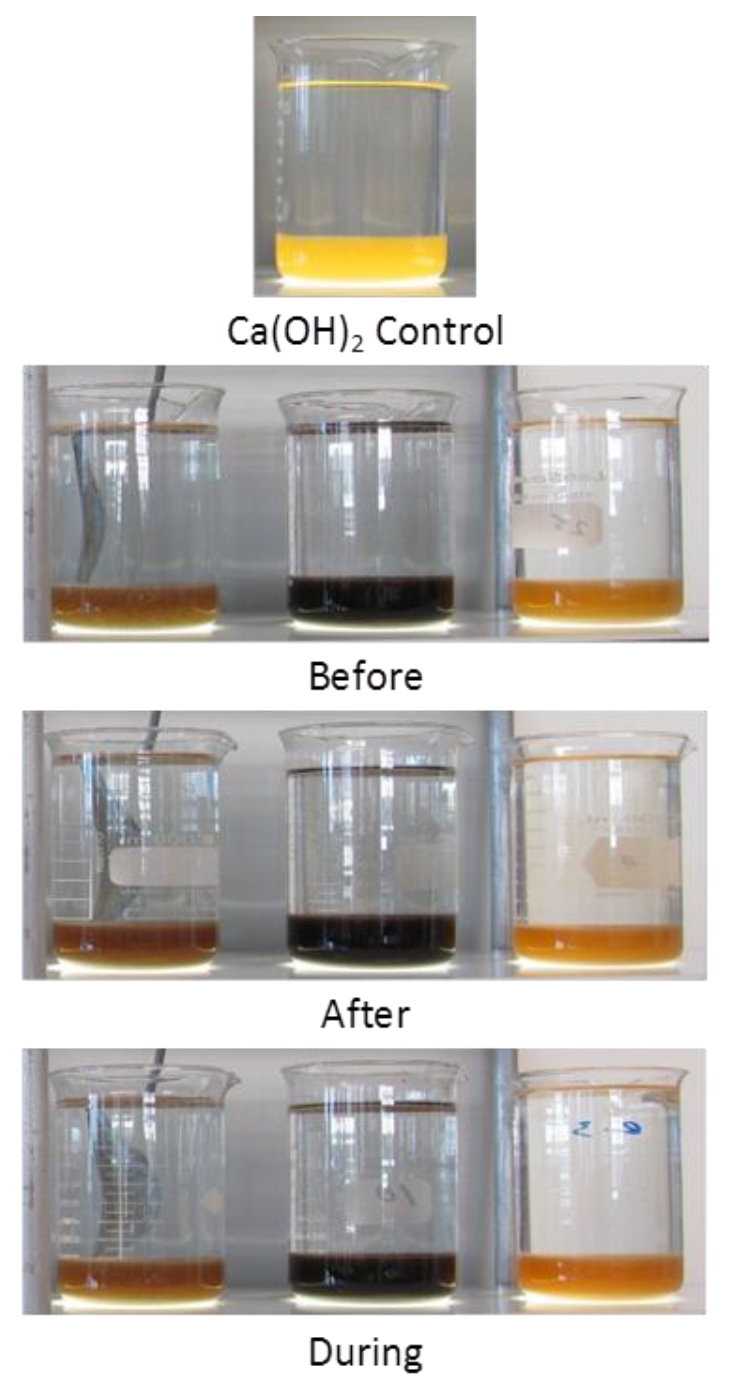

Figure 6-5: $\mathrm{Ca}(\mathrm{OH})_{2}$ neutralised supernatant (L to R: SEHA20, SEHA100, AHA20)

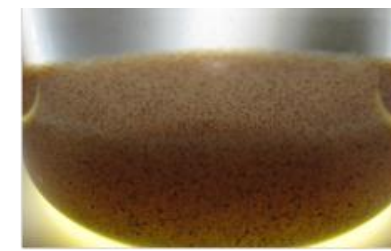

Before

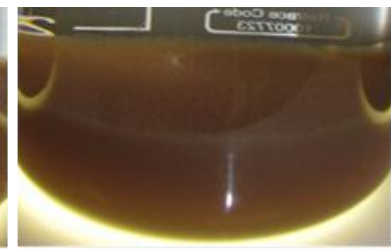

After

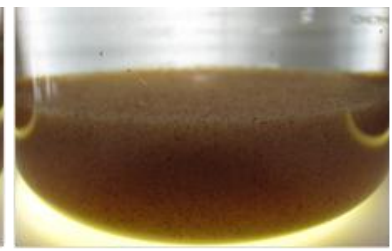

During

Figure 6-6: Comparison of $\mathrm{Ca}(\mathrm{OH})_{2}$ neutralised SEHA20 sludge 
HS - Water Quality

Chen et al. (2007) showed that Ca binding to HS was significantly greater than Na. The slightly elevated turbidity and SS may have been due to Ca complexation of HS. The complexation of Ca by HS would neutralise the negative charge of HS carboxylic groups, preventing adsorption to positively charged hydroxide precipitates. The slightly elevated turbidity and SS may also have been due to a fraction of the $30 \mathrm{mg}$ unreacted $\mathrm{Ca}(\mathrm{OH})_{2}$ (discussed in section 5.1) remaining in suspension.

Figure 6-6 suggests that HS precipitation and metal oxide adsorption mechanisms may remove HS from samples dosed before and during neutralisation, as HS precipitates were still visible in the settled sludge for these conditions. The precipitation of HS and subsequent adsorption to metal hydroxides is a $\mathrm{pH}$ controlled process and occurs somewhat independently of the alkalinity $\left(\mathrm{NaOH}\right.$ or $\left.\mathrm{Ca}(\mathrm{OH})_{2}\right)$ reagent used.

Figure 6-5 and Figure 6-6 show HS addition after neutralisation produced a homogenously brown sludge and low supernatant discolouration. The clear and colourless supernatant suggests $\mathrm{HS}$ was removed by an additional mechanism not available in $\mathrm{NaOH}$ neutralisation. The Ca concentration of $\mathrm{Ca}(\mathrm{OH})_{2}$ neutralised samples was approximately $380 \mathrm{mg} / \mathrm{L}$, due primarily to $\mathrm{Ca}(\mathrm{OH})_{2}$ dissolution. Intermolecular bridging of $\mathrm{HA}$ by $\mathrm{Ca}$ complexation results in rapid formation and growth of HA aggregates, at circum neutral pH (Chen and Elimelech 2007), resulting in HS incorporation into sludge. This removal mechanism was not available under $\mathrm{NaOH}$ neutralisation as $\mathrm{Na}$ is monovalent. 
HS - Water Quality

\subsubsection{MSS Water Quality}

This section discusses the results of the Mangatini Stream-sump System (MSS) conditions from the $\mathrm{HS}$ jar testing, neutralised by $\mathrm{CaCO}_{3}$. One turbidity and SS sample was taken for each sedimentation period (2, 6, $24 \mathrm{hrs}, 3,5,7$ days). Therefore the figures in this section show the kinetic water quality of the MSS conditions.

\subsubsection{1 $\mathrm{CaCO}_{3}$ Water Quality}

Figure 6-7 shows the turbidity and $\mathrm{SS}$ of $\mathrm{CaCO}_{3}$ neutralised conditions. Raw data are included in Appendix 3.
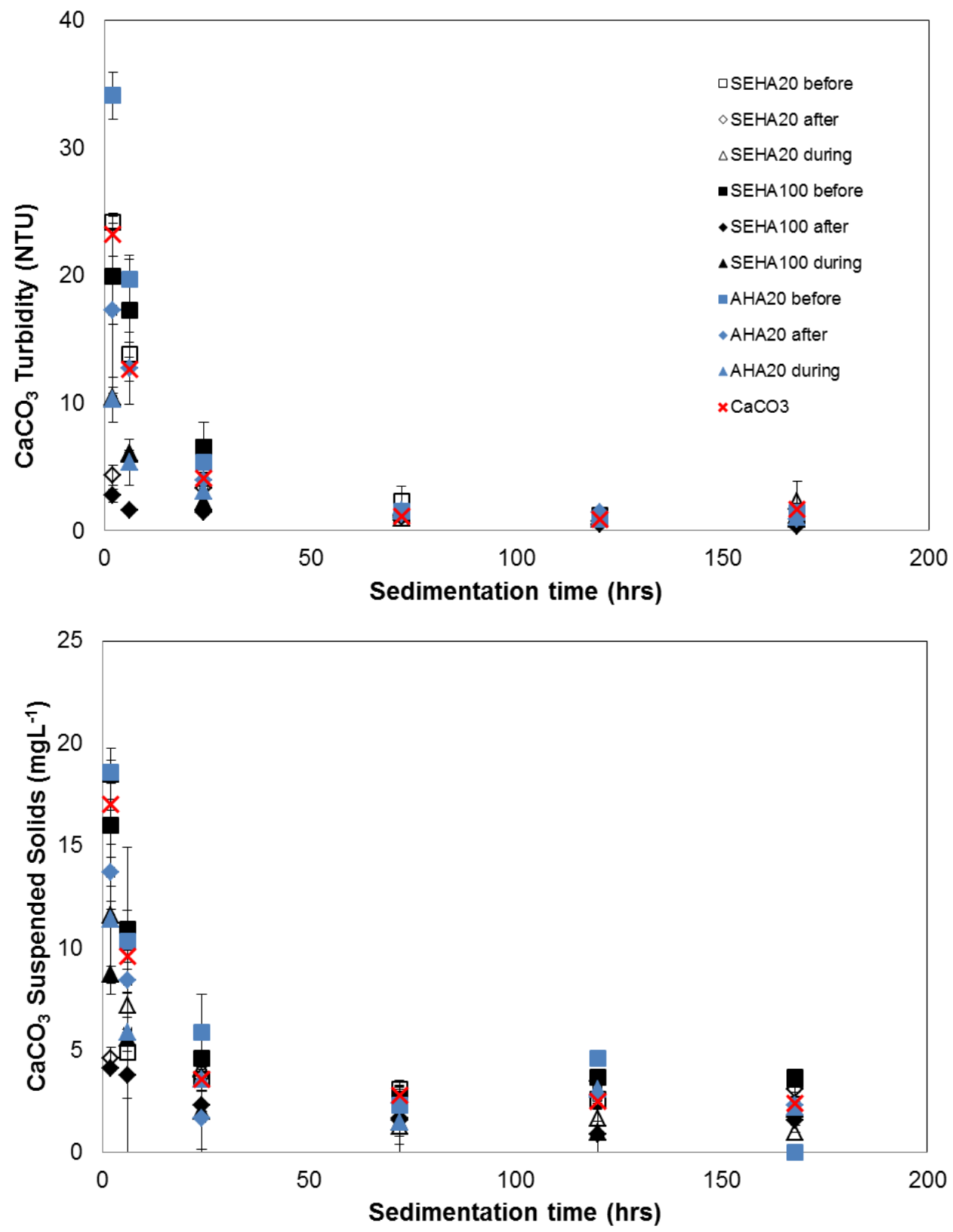

Figure 6-7: $\mathrm{CaCO}_{3}$ neutralised water quality . Standard deviation error $(n=2)$. 
HS - Water Quality

The water quality of $\mathrm{CaCO}_{3}$ neutralised conditions showed a strong dependence on time, particularly over the first 24 hours. The highest turbidity and SS values occurred at the first, two hour sedimentation time. The $\mathrm{CaCO}_{3}$ control samples resulted in maximum turbidity and SS of $23 \mathrm{NTU}$ and $17.0 \mathrm{mg} / \mathrm{L}$, respectively. The turbidity and SS decreased over time to a steady $1 \mathrm{NTU}$ and $2.5 \mathrm{mg} / \mathrm{L}$, respectively, after 72 hours sedimentation. The decrease in SS and turbidity of control samples is typical of discrete particle settling with a very low settling velocity (Tchobanoglous, Burton et al. 2003).

Figure 6-7 shows HS does enhance the removal of unreacted $\mathrm{CaCO}_{3} \mathrm{SS}$, when added at the correct time. The results for SEHA100 clearly show a hierarchy where SS removal by HS addition after- $>$ during- $>$ before-neutralisation $\approx$ control with final SS concentrations 4.1, 8.7, 16 and $17 \mathrm{mg} / \mathrm{L}$ respectively. The hierarchy holds for turbidity and SS for both SEHA concentrations. However, AHA results showed a hierarchy of during- > after- > before-neutralisation $\approx$ control, for turbidity and SS. Figure 6-8 shows the relatively clear supernatants of SEHA dosed during- and after-neutralisation conditions, while the supernatants of AHA and SEHA before-neutralisation appear cloudy. 
HS - Water Quality

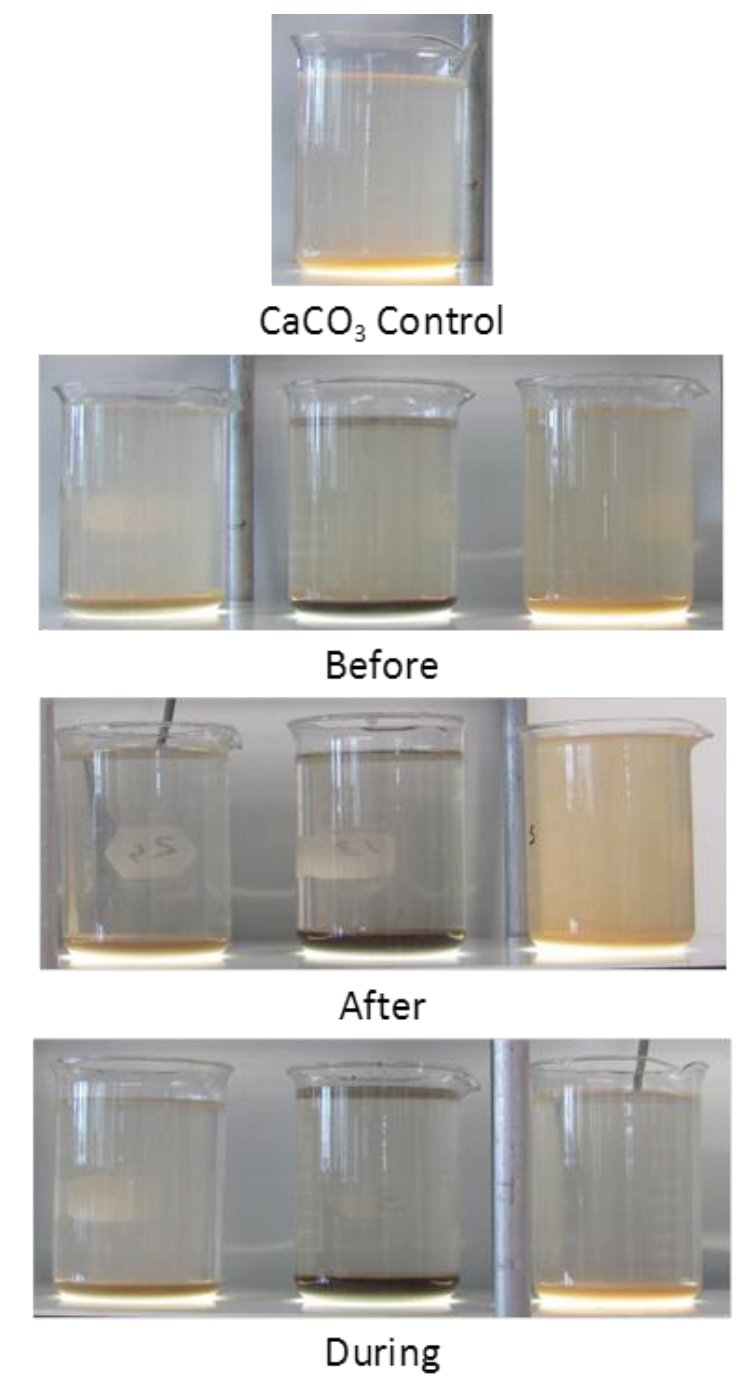

Figure 6-8: $\mathrm{CaCO}_{3}$ neutralised supernatant (L to R: SEHA20, SEHA100, AHA20)

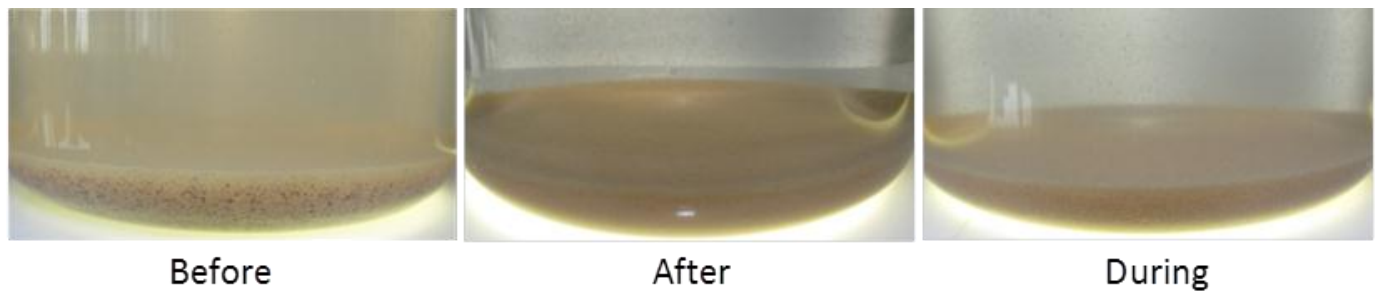

Figure 6-9: Comparison of $\mathrm{CaCO}_{3}$ neutralised SEHA20 sludge

The total MSS neutralisation dose was $1800 \mathrm{mg} \mathrm{CaCO}_{3} / \mathrm{L}$ as determined in section 5.2. At the two hour supernatant sampling, the $\mathrm{pH}$ was approximately 6.4 , corresponding to a neutralisation efficiency of $43 \%$, (assuming $780 \mathrm{mg} / \mathrm{L} \mathrm{CaCO}_{3}$ alkalinity was required to neutralise AMD to $\mathrm{pH}$ 6.4, as determined by $\mathrm{NaOH}$ neutralisation). Therefore, after two hours sedimentation $1020 \mathrm{mg}$ of $\mathrm{CaCO}_{3}$ remained unreacted. The majority of the unreacted $\mathrm{CaCO}_{3}$ settled as sludge, however a fraction of $\mathrm{CaCO}_{3}$ remained suspended, 
HS - Water Quality

resulting in high supernatant turbidity and SS. Figure 6-8 shows the $\mathrm{CaCO}_{3}$ SS resulted in cloudy supernatants for all HS dosed conditions after 30min sedimentation.

Settlement of unreacted alkaline reagent appears to be the dominant factor affecting $\mathrm{CaCO}_{3}$ neutralised water quality; unlike $\mathrm{NaOH}$ and $\mathrm{Ca}(\mathrm{OH})_{2}$ neutralised scenarios, where settling of the precipitated metal hydroxides was the major factor affecting water quality. Thus, the processes affecting the sedimentation of metal hydroxides become secondary in terms of water quality to the processes affecting the sedimentation of $\mathrm{CaCO}_{3}$ particulate.

Addition of HS after-neutralisation resulted in a homogenously brown sludge, suggesting good HS incorporation. The PZC of calcite varies, but typically occurs at $\mathrm{pH}$ greater than 8 (Wolthers, Charlet et al. 2008). Thus, when added after neutralisation ( $\mathrm{pH}$ 6) negatively charged $\mathrm{HS}$ and positively charged $\mathrm{CaCO}_{3}$ particles may aggregate. These particles formed floc and settled from suspension, resulting in a relatively clear supernatant. Competition from cations for adsorption sites on HS would probably be minimal due to the significant $\mathrm{CaCO}_{3}$ concentration.

The before-neutralisation photo on Figure 6-9 shows the formation of a two-strata sludge. The bottom layer comprised precipitated HS dispersed through larger $\mathrm{CaCO}_{3}$ particles. The upper layer was formed by gradual deposition and accumulation of finer $\mathrm{CaCO}_{3}$ particulate, with a lower settling velocity. The acid precipitated HS is still clearly visible in the before-neutralisation condition. The acid precipitation of HS was shown to be irreversible at circum neutral $\mathrm{pH}$ in section 6.2.1.1. Thus, incorporation of the HS into floc when added before neutralisation would mean no residual HS was available to flocculate residual $\mathrm{CaCO}_{3} \mathrm{SS}$ after neutralisation, resulting in similar values of turbidity and SS for the before-neutralisation condition to the control.

The turbidity and SS of the during-neutralisation conditions were lower than the beforeneutralisation condition but higher than the after-neutralisation condition. This suggests some residual HS was available in the during-neutralisation supernatant for removal of $\mathrm{CaCO}_{3} \mathrm{SS}$.

Two observations were made from the 72 hour sedimentation dataset on Figure 6-7. Firstly, beyond 72 hours the turbidity and SS were stable, suggesting the equilibrium water quality has been reached. Secondly, the turbidity and SS for HS dosed conditions after 72 hours were relatively similar to the $\mathrm{CaCO}_{3}$ control, suggesting that although 
HS - Water Quality

addition of $\mathrm{HS}$ during and after neutralisation increased the rate of $\mathrm{CaCO}_{3} \mathrm{SS}$ removal, the total SS removal after 72 hours was unaffected. As SS removal rates are an important parameter for design of water treatment facilities this observation was further investigated in the MSS polymer application.

The optimal performance of SEHA as a polymer when added after AMD neutralisation by $\mathrm{CaCO}_{3}$ has interesting engineering implications. Siting of the $\mathrm{CaCO}_{3}$ dosing plant must occur significantly upstream of the sedimentation reservoir, to provide sufficient instream residence time for neutralisation. Incorporation of $\mathrm{HS}$ dosing into the $\mathrm{CaCO}_{3}$ dosing plant would result in low HS polymer performance, due to relatively low AMD neutralisation early in the treatment phase; with expected polymer performance comparable to HS dosing during-neutralisation. Thus, a separate HS dosing plant would require construction further downstream.

Figure 6-7 showed the HS polymer effect was greatest when added after $\mathrm{CaCO}_{3}$ neutralisation. The optimum HS dosing site therefore would be close to the point of discharge into the sedimentation reservoir, provided sufficient mixing and complexation/adsorption of HS could be achieved. At sites where the sedimentation reservoir is man-made, there would probably be good access to the stream close to the discharge point into the reservoir. Thus, construction of a HS dosing system, possibly incorporating HS pumping upstream to increase mixing time, should be feasible for addition of HS after neutralisation. 
HS - Water Quality

\subsection{MSS Polymer Application}

The enhanced removal of $\mathrm{CaCO}_{3} \mathrm{SS}$ by HS warranted further investigation. Therefore a set of jar test experiments were conducted at a range of HS concentrations to determine the optimal dose rate to reduce residual $\mathrm{CaCO}_{3} \mathrm{SS}$ and turbidity, with respect to total removal and removal efficiency. The performance of HS was compared to the commercially available Crystalfloc polymer. The cost: benefit analysis in section 8.1.1 was completed using the optimal dose rates identified in this section.

\subsubsection{SEHA Water Quality}

Figure 6-10 shows the turbidity and $\mathrm{SS}$ of $\mathrm{CaCO}_{3}$ neutralised AMD at different HS doses. Raw data are included in Appendix 4.

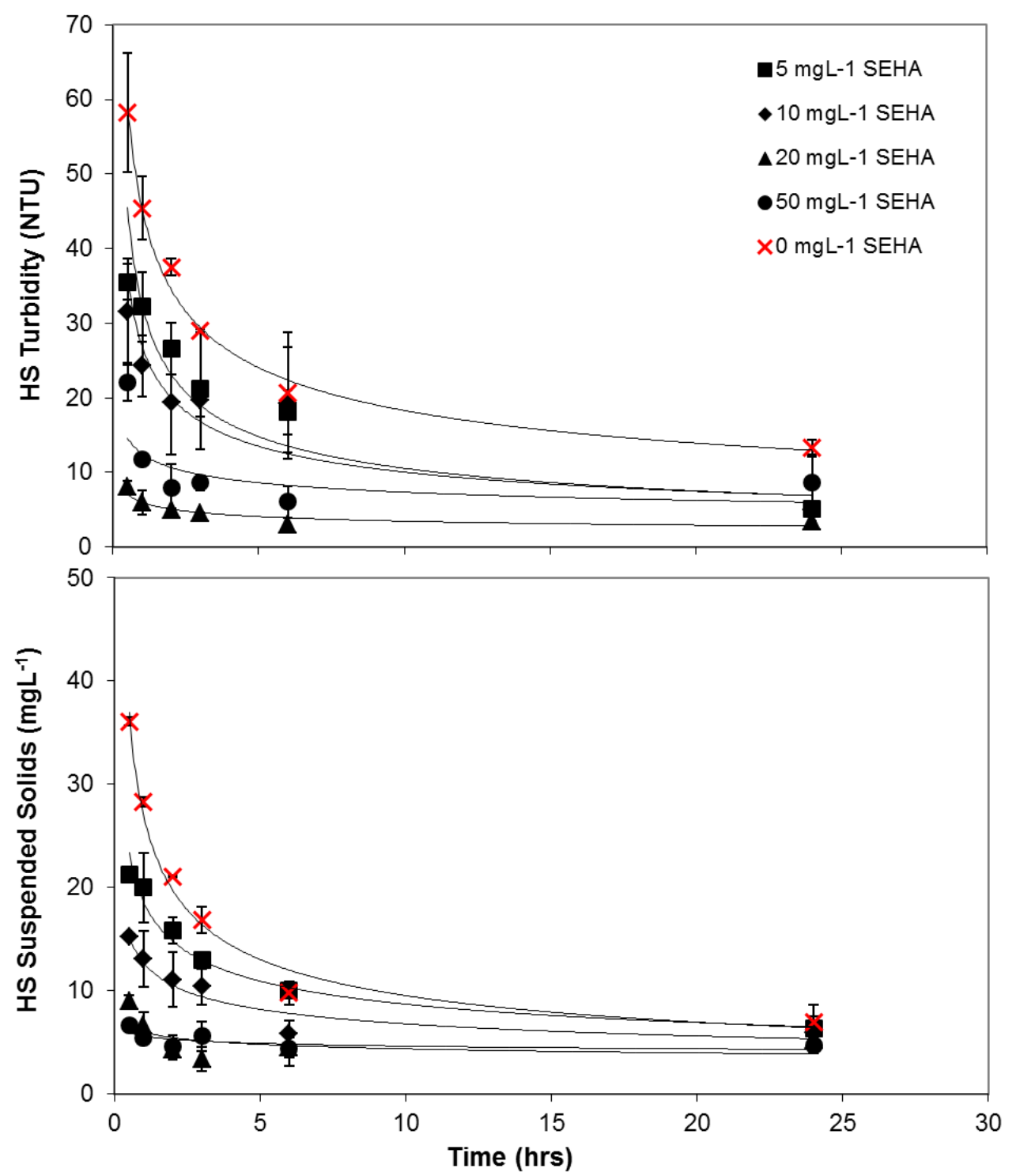

Figure 6-10: HS polymer effect on water quality . Standard deviation error $(\mathrm{n}=2)$. 
HS - Water Quality

The first data point was collected after 30 minutes sedimentation. At this time the majority of floc had settled and a clear interface between sludge zone and supernatant had formed. The initial control turbidity and SS were $58.3 \mathrm{NTU}$ and $36.1 \mathrm{mg} / \mathrm{L}$, respectively. As discussed in section 6.2.2.1, the turbidity and $\mathrm{SS}$ was due to unreacted $\mathrm{CaCO}_{3} \mathrm{SS}$. NB: the initial control turbidity and SS were higher in the polymer trials as the first sample was collected after 30 minutes sedimentation as opposed to 2 hours sedimentation in the MSS water quality section.

Figure 6-10 shows the initial SS of HS amended samples were 21.3, 15.2, 9.0 and 6.7 $\mathrm{mg} / \mathrm{L}$ for $5,10,20$ and $50 \mathrm{mg} / \mathrm{L}$ SEHA, respectively. This immediately identifies a negative correlation between HS concentration and SS, for the HS doses investigated. The turbidity and SS reduced throughout the sedimentation period under all conditions. This shows that the residual SS in the supernatant would naturally settle out of suspension over 6 to 24 hours.

Figure 6-10 suggests that HS substantially enhances the SS removal rate but not the total SS removal at equilibrium. For example, the SS of the $50 \mathrm{mg} / \mathrm{L}$ HS dose was $19 \%$ of control after two hours sedimentation. After 24 hours sedimentation the SS of the 50 $\mathrm{mg} / \mathrm{L}$ HS dose increased to $67 \%$ of the control. The results for turbidity showed a similar trend.

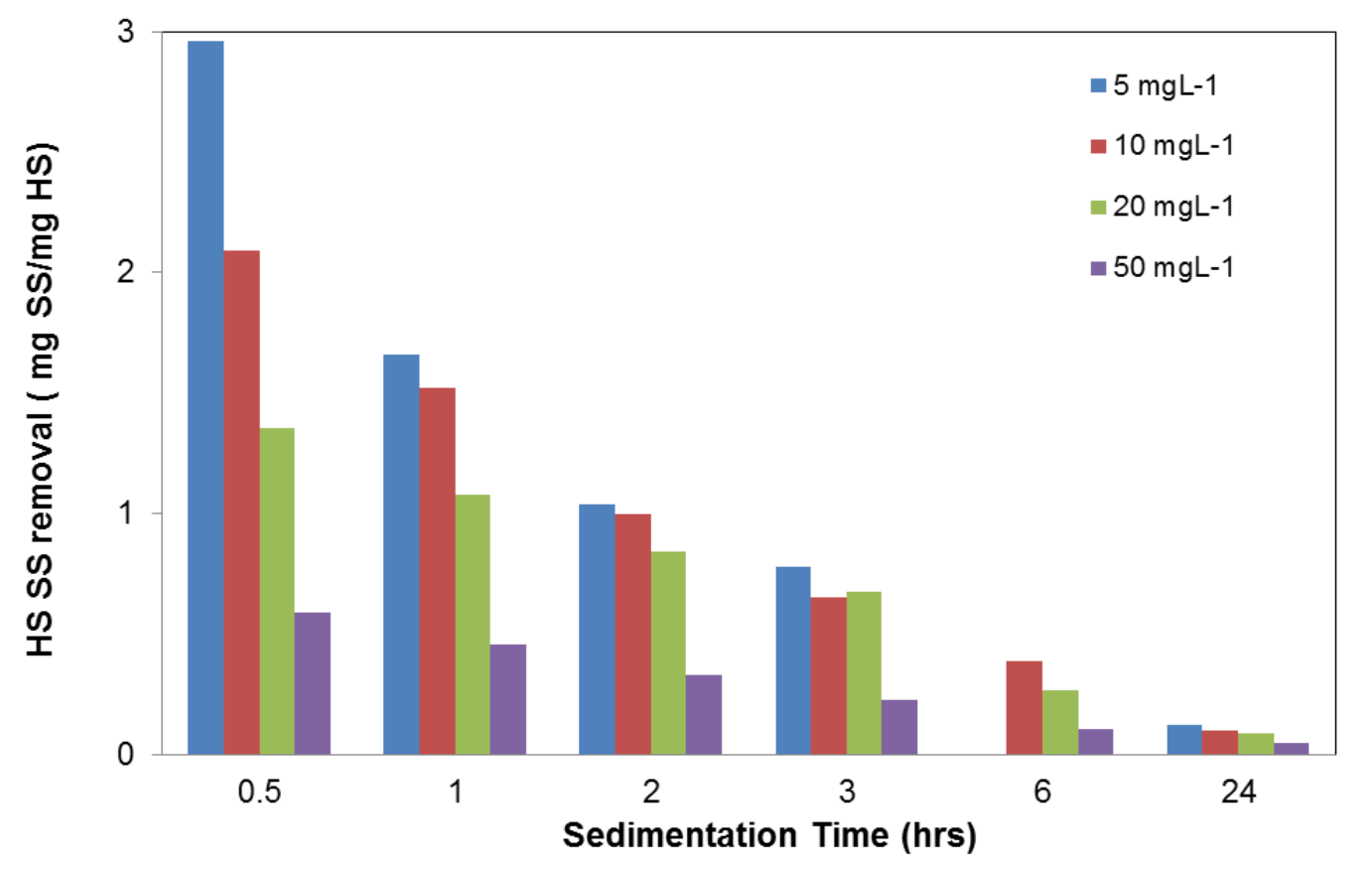

Figure 6-11: SS removal efficiency of $\mathrm{HS}$ 
HS - Water Quality

Figure 6-11 shows the removal of SS per mg HS dose, which may be used as an indicator of SS removal efficiency. The removal efficiency was determined based on the additional SS removal achieved for each HS dose, using Equation 6-1.

$$
e=\frac{S S_{c}-S S_{H S}}{H S}
$$

Equation 6-1

HS-SS removal efficiency

Where, e is the removal efficiency ( $\mathrm{mg} \mathrm{SS} / \mathrm{mg} \mathrm{HS}$ )

$\mathrm{SS}_{\mathrm{c}}$ is the control SS at the given sedimentation time (mg SS/L)

$\mathrm{SS}_{\mathrm{HS}}$ is the HS dosed SS (mg SS/L)

HS is the HS dose concentration (mg HS/L)

The removal efficiency decreased over time for all HS doses as the control SS approaches equilibrium. Overdosing is shown by a decreasing SS removal per mg HS added. Figure 6-11 shows the greatest removal efficiency occurred using $5 \mathrm{mg} / \mathrm{L} \mathrm{HS}$, particularly for the initial $30 \mathrm{~min}$ sampling point. The $20 \mathrm{mg} / \mathrm{L}$ dose achieved lower SS concentrations sooner (Figure 6-10) without significantly compromising the removal efficiency (Figure 6-11). Therefore, the recommended HS dose would be $20 \mathrm{mg} / \mathrm{L}$.

Figure 6-11 shows similar removal efficiencies after two hours sedimentation for 5, 10 and $20 \mathrm{mg} / \mathrm{L}$ HS dosing. This suggests that when under-dosed, HS will facilitate SS removal proportional to the HS dose. This property would allow HS to perform in a dynamic environment where other polymers may fail when under-dosed below the critical concentration for flocculation, which is discussed further in the next section. 
HS - Water Quality

\subsubsection{Crystalfloc Polymer Comparison}

This section discusses the results of polymer trials for turbidity and SS removal. The results from cationic Crystalfloc B570 are discussed first, followed by anionic Crystalfloc B610.

Both the anionic and cationic polymers were a low molecular weight grade, with molar mass between 1 and 3 million $\mathrm{g} / \mathrm{mol}$. When prepared in a stock solution at $0.1 \%$, both polymers were below saturation. Precipitates were not observed for at least two days after stock solution preparation, suggesting polymer did not degrade over the testing period.

\subsubsection{Crystalfloc B570 Polymer}

Figure 6-12 shows the turbidity and SS results of the cationic Crystalfloc B570 polymer trials. Raw data are included in Appendix 4.

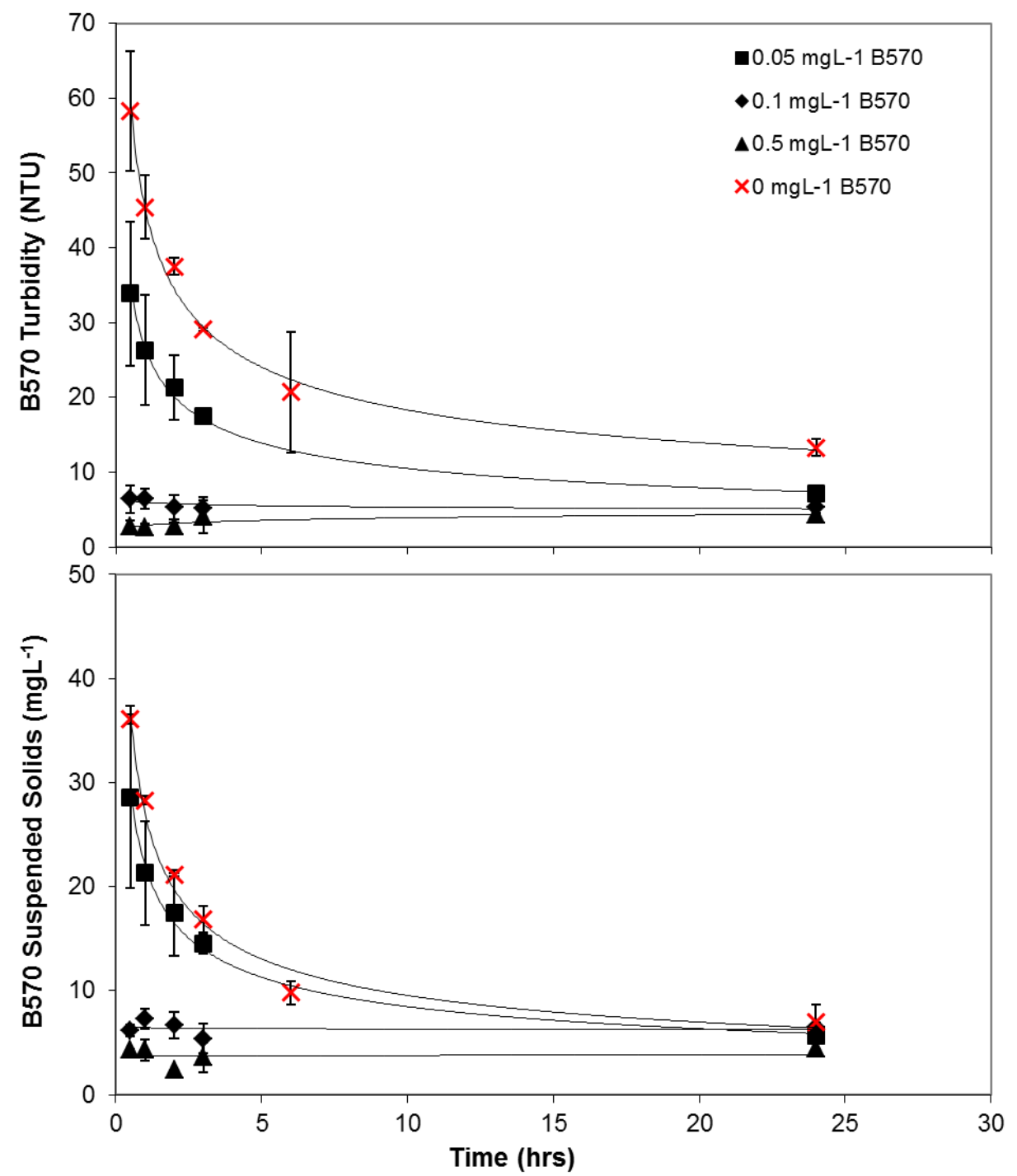

Figure 6-12: B570 polymer effect on water quality. Standard deviation error $(\mathrm{n}=2)$. 
HS - Water Quality

The HS control data in section 6.3.1 were used for the Crystalfloc control. Figure 6-12 shows initial SS of the B570 dosed AMD as 28.6, 6.2 and $4.3 \mathrm{mg} / \mathrm{L}$ for $0.05,0.1$ and 0.5 mg/L B570 doses, respectively, identifying a negative correlation between B570 dose and SS. Similarly to HS dosing, the B570 polymer increases the rate of SS removal, but not the total SS removal. For the $0.1 \mathrm{mg} / \mathrm{L}$ B570 dose, the SS at 2 hours was $17 \%$ of the control and increased to $93 \%$ of the control at equilibrium. The SS concentration for both the 0.1 and $0.5 \mathrm{mg} / \mathrm{L} \mathrm{B} 570$ doses were essentially at equilibrium at the initial sampling point, corresponding to a SS concentration $12-17 \%$ of the control. At equilibrium, the B570 dosed SS concentrations (4.4-6.5 mg/L) were essentially the same as those for HS dosed (4.7-6.5 mg/L) AMD.

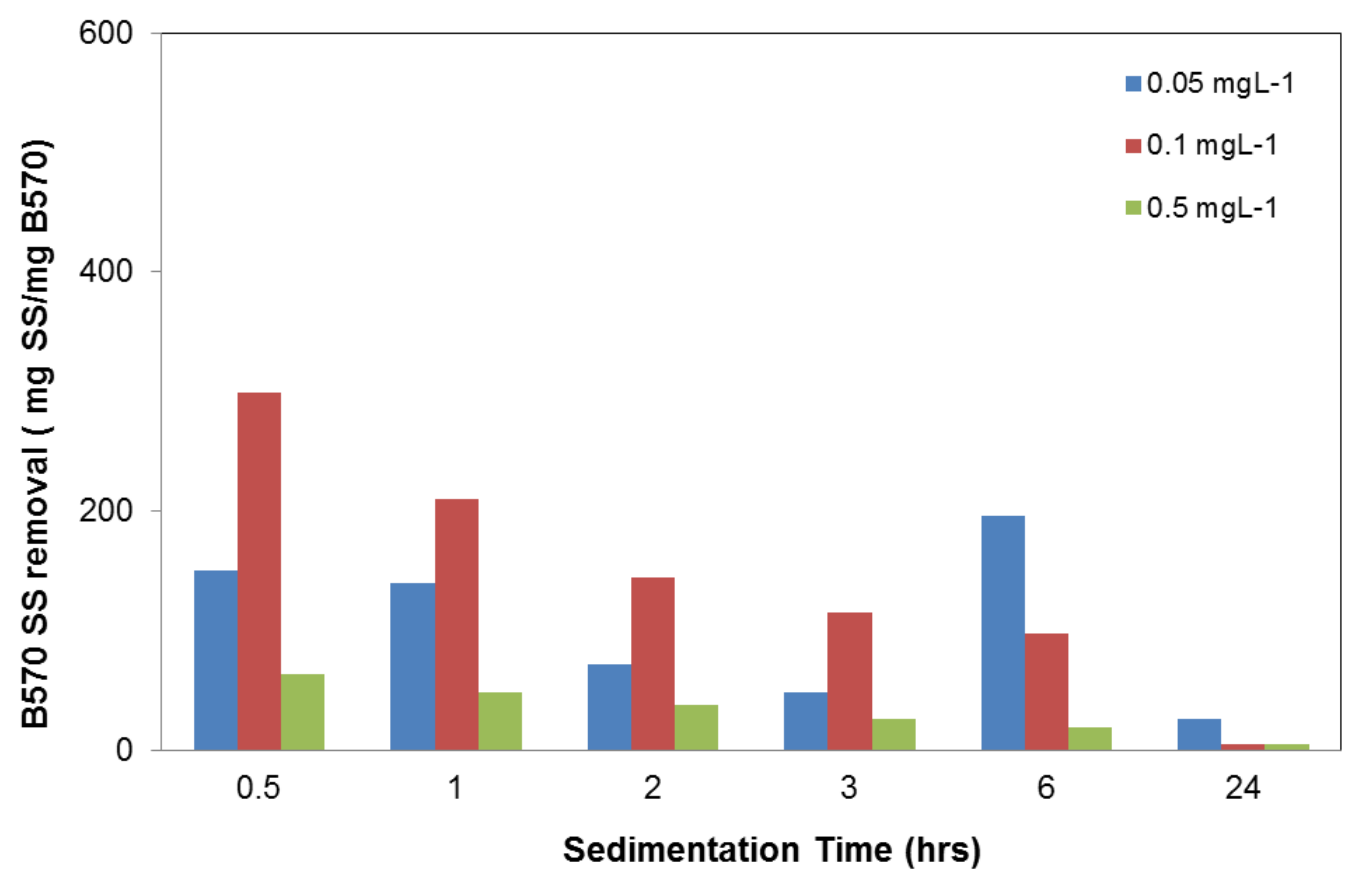

Figure 6-13: SS removal efficiency of B570

Figure 6-13 shows the removal efficiency of B570 for SS. With respect to removal efficiency, $0.1 \mathrm{mg} / \mathrm{L}$ B570 appears to be the optimal dose, as it achieves the greatest SS removal per mg HS dose at all sedimentation times, except 6 and 24 hours. The $0.5 \mathrm{mg} / \mathrm{L}$ B570 dose was excessive, resulting in decreased removal efficiency.

Dosing with $0.05 \mathrm{mg} / \mathrm{L}$ B570 resulted in a lower removal efficiency than $0.1 \mathrm{mg} / \mathrm{L}$. At low concentrations polymers bind to themselves and do not enhance flocculation. As polymer concentration is increased to the critical concentration individual polymers contact each other, resulting in floc aggregation (SNF FLOERGER). The lower removal 
HS - Water Quality

efficiency at the $0.05 \mathrm{mg} / \mathrm{L}$ B570 dose suggests the critical concentration to trigger flocculation is approximately $0.1 \mathrm{mg} / \mathrm{L} \mathrm{B570.}$

\subsubsection{Crystalfloc B610 Polymer}

Figure 6-14 shows the turbidity and SS results of the anionic Crystalfloc B610 polymer trials. Raw data are included in Appendix 4.
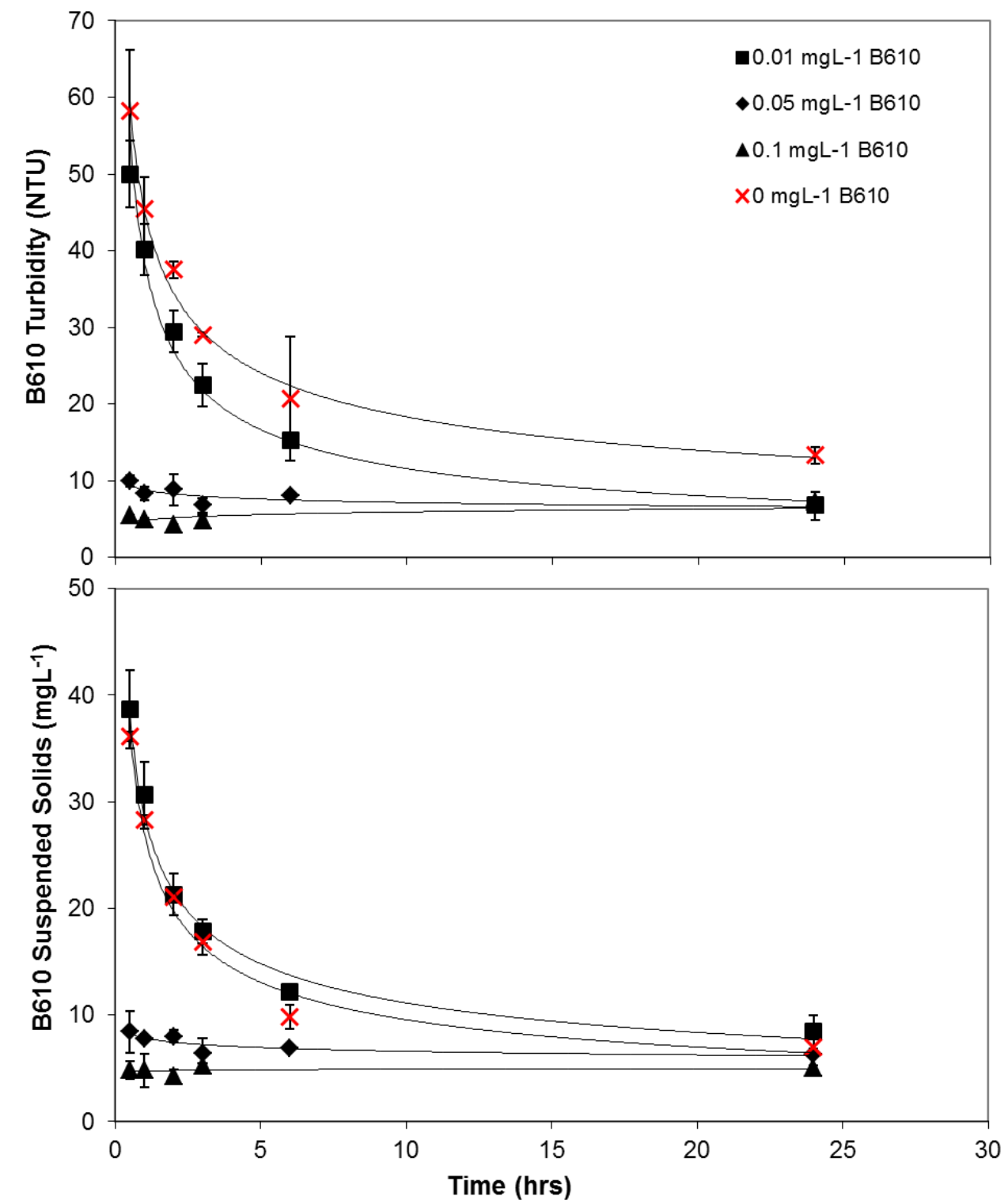

Figure 6-14: B610 polymer effect on water quality. Standard deviation error (n=2).

Figure 6-14 shows the initial SS of the B610 dosed AMD as 38.7, 8.4 and $4.8 \mathrm{mg} / \mathrm{L} \mathrm{SS}$ for $0.01,0.05$ and $0.1 \mathrm{mg} / \mathrm{L} \mathrm{B610}$, again indicating a negative correlation between B610 dose and SS. Similarly to HS and B570 dosing, the B610 polymer increases the rate of SS 
HS - Water Quality

removal, but not the total SS removal; the SS of the $0.1 \mathrm{mg} / \mathrm{L} \mathrm{B} 610$ dose was $13 \%$ of the control after $30 \mathrm{~min}$ and increased to $71 \%$ of the control at equilibrium.

The SS of 0.05 and $0.1 \mathrm{mg} / \mathrm{L} \mathrm{B610} \mathrm{doses} \mathrm{were} \mathrm{at} \mathrm{equilibrium} \mathrm{when} \mathrm{the} \mathrm{initial} \mathrm{sample} \mathrm{was}$ taken, corresponding to 13 to $17 \%$ of the initial control SS. At equilibrium, the B610 dosed SS concentrations $(5.0-8.4 \mathrm{mg} / \mathrm{L})$ were slightly higher than those for HS and B570 dosed (4.4 - $6.5 \mathrm{mg} / \mathrm{L})$ AMD.

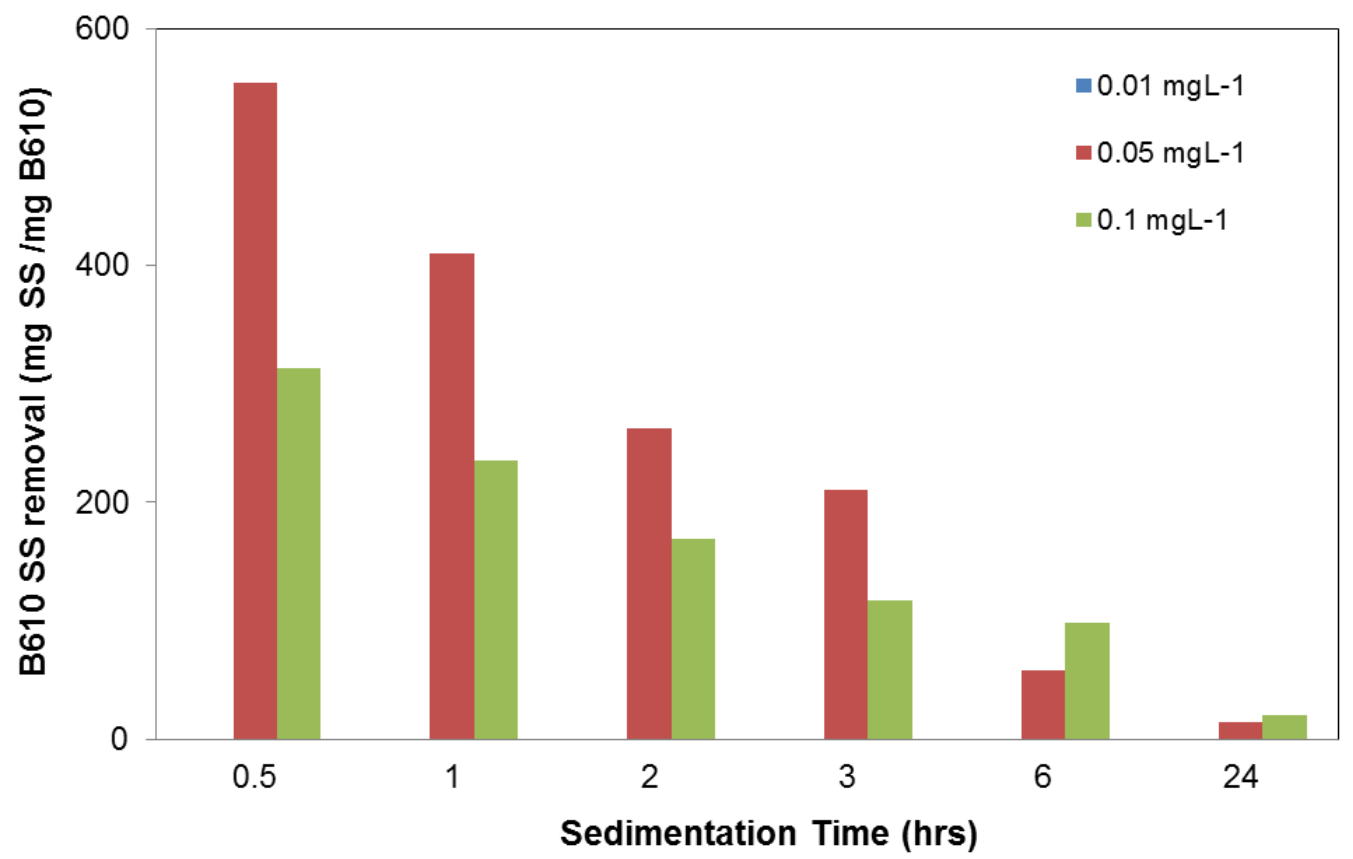

Figure 6-15: SS removal efficiency of B610

Figure 6-15 shows the SS removal efficiency of B610 dosed AMD. The optimum B610 dose for removal efficiency was $0.05 \mathrm{mg} / \mathrm{L}$. The $0.01 \mathrm{mg} / \mathrm{L}$ data have been omitted as they were negative (due to higher SS concentrations for $0.01 \mathrm{mg} / \mathrm{L} \mathrm{B} 610$ dosed conditions than the control on Figure 6-14). Excessive B610 dosing leads to lower removal efficiency; as shown for the $0.1 \mathrm{mg} / \mathrm{L}$ condition.

The anionic (B610) polymer removed $\mathrm{CaCO}_{3} \mathrm{SS}$ with the greatest efficiency. At $\mathrm{pH} \sim 6$, calcite would be weakly protonated $(\mathrm{PZC}<8)$. Therefore, unreacted $\mathrm{CaCO}_{3}$ would have a greater affinity for the anionic B610 polymers. Minimum turbidity and SS were achieved by significantly lower Crystalfloc doses (30 - 65 times lower) than SEHA. The efficiency of SEHA as a polymer compared to Crystalfloc is investigated further in Chapter 8 (Feasibility/Markets). 
HS - Water Quality

\subsection{Summary}

The water quality of HS dosed AMD supernatants varied depending on the dose order and alkalinity reagent used. Different mechanisms (precipitation, co-precipitation, and adsorption) operated under different HS dose conditions and affected the final water quality.

The addition of HS before and during neutralisation of AMD by $\mathrm{NaOH}$ resulted in supernatant turbidity and SS below detection limits. Acid precipitated HS may provide a negatively charged nucleation site for adsorption of positively charged precipitating metal hydroxides, resulting in the aggregation of floc. Thus, all HS and precipitating metals appear to be incorporated into AMD sludge resulting in a clear supernatant. However, when added after neutralisation (at $\mathrm{pH}$ 7) the surface charge on both HS and precipitated metals was negative, resulting in poor incorporation of HS into sludge. The water quality of the SEHA100 after-neutralisation condition was particularly poor with turbidity and SS of $24.4 \mathrm{NTU}$ and $18.4 \mathrm{mg} / \mathrm{L}$, respectively. The absence of a multi-valent bridging cation (monovalent $\mathrm{Na}$ was dominant cation) was identified as potentially critical in the lack of HS incorporation into AMD sludge.

Similar trends were observed for the addition of HS before and during neutralisation by $\mathrm{Ca}(\mathrm{OH})_{2}$. The precipitated HS acted as nucleation sites, triggering flocculation. In contrast to the results for $\mathrm{NaOH}$ neutralised AMD, the addition of $\mathrm{HS}$ after neutralisation by $\mathrm{Ca}(\mathrm{OH})_{2}$ resulted in relatively good water quality; with turbidity below detection limits and SS of $3.0 \mathrm{mg} / \mathrm{L}$, for the SEHA100 after-neutralisation condition. The presence of divalent $\mathrm{Ca}(\sim 400 \mathrm{mg} / \mathrm{L})$ as a product of $\mathrm{Ca}(\mathrm{OH})_{2}$ dissociation facilitated inter-particle bridging of HS molecules (Chen and Elimelech 2007), resulting in the removal of a high degree of residual HS. Higher values of SS (up to $5 \mathrm{mg} / \mathrm{L}$ ) were recorded for $\mathrm{Ca}(\mathrm{OH})_{2}$ neutralised AMD, possibly caused by a small amount of residual alkalinity due to the $96 \%$ neutralisation efficiency of $\mathrm{Ca}(\mathrm{OH})_{2}$.

The low dissolution efficiency of $\mathrm{CaCO}_{3}(42 \%)$ at $\mathrm{pH} 6$ resulted in up to $1040 \mathrm{mg} / \mathrm{L}$ of undissolved residual $\mathrm{CaCO}_{3}$. This residual $\mathrm{CaCO}_{3}$ impacted the water quality with turbidity and SS of control samples significantly elevated at 23 NTU and $17 \mathrm{mg} / \mathrm{L}$, respectively, after two hours sedimentation. Addition of HS after neutralisation resulted in a significant increase in the rate of turbidity and SS removal. The turbidity and SS of the SEHA100 after-neutralisation condition were essentially at equilibrium levels at the 
HS - Water Quality

first sample collection point, two hours into the sedimentation period. The HS was therefore acting as a polymer. Addition of HS before neutralisation resulted in HS precipitation and incorporation into sludge, preventing the HS polymer phenomena. The water quality of $\mathrm{CaCO}_{3}$ neutralised conditions was therefore governed by $\mathrm{CaCO}_{3}$ particulate-HS interactions not metal hydroxide-HS interactions.

The performance of SEHA as a polymer to reduce the turbidity and $\mathrm{SS}$ of $\mathrm{CaCO}_{3}$ neutralised AMD was investigated. The optimum SEHA dose was identified as $20 \mathrm{mg} / \mathrm{L}$ for the $\mathrm{CaCO}_{3}$ polymer effect, although this dose would vary depending on AMD chemistry on site. The performance of SEHA was compared with two Crystalfloc B570 and B610 polymers, which achieved the same water quality at dose rates of 0.1 and 0.05 $\mathrm{mg} / \mathrm{L}$ respectively. 



\section{Chapter 7. HS - Metal Removal}

The HS - metal removal chapter is divided into three sections.

Section 1: Raw AMD. This section states the concentration of relevant metals in the raw AMD.

Section 2: Metal Removal. This section discusses the residual metal concentration for both the BTP and MSS application. The results of trials on the impact of HS dose order and concentration on metal concentration for $\mathrm{NaOH}, \mathrm{Ca}(\mathrm{OH})_{2}$ and $\mathrm{CaCO}_{3}$ neutralised AMD are discussed. The HS dosed conditions are compared to controls to identify the HS enhancement in metal removal.

Section 3: Equilibrium Speciation. This section discusses the experimental and theoretical speciation modelling data. 


\subsection{Raw AMD}

Raw AMD samples were analysed throughout the experimentation period as a quality control measure to identify any decay in metal concentration during storage. Fifteen AMD samples were analysed in total. Both $\mathrm{Cd}$ and $\mathrm{Pb}$ showed a small concentration decrease with respect to time (probably due to adsorption to container walls or sediments (Pope, Newman et al. 2010a)), while Ni, $\mathrm{Cu}$ and $\mathrm{Zn}$ concentrations were constant.

The concentration of $\mathrm{Cd}$ decreased from an initial 0.029 to $0.005 \mathrm{mg} / \mathrm{L}$ over the experimentation period. Control neutralised samples of fresh AMD resulted in Cd concentrations of $0.002 \mathrm{mg} / \mathrm{L}$ for both $\mathrm{NaOH}$ and $\mathrm{Ca}(\mathrm{OH})_{2}$ controls. Control neutralised samples from the end of the experimental period resulted in $\mathrm{Cd}$ concentrations of 0.002 and $0.005 \mathrm{mg} / \mathrm{L}$ for $\mathrm{NaOH}$ and $\mathrm{Ca}(\mathrm{OH})_{2}$ neutralised AMD. These observations suggest a decreasing raw AMD Cd concentration did not result in lower Cd concentrations after neutralisation.

The raw AMD concentration of $\mathrm{Pb}$ decreased from 0.088 to $0.044 \mathrm{mg} / \mathrm{L}$. Control samples neutralised using fresh $\mathrm{AMD}$ resulted in $\mathrm{Pb}$ concentration of $0.048 \mathrm{mg} / \mathrm{L}$ and below detection limit $(0.024 \mathrm{mg} / \mathrm{L})$ for $\mathrm{NaOH}$ and $\mathrm{Ca}(\mathrm{OH})_{2}$ neutralised samples respectively. Control samples generated from older AMD were at or below the detection limit. This data suggests a decreasing raw $\mathrm{Pb}$ concentration resulted in lower neutralised AMD $\mathrm{Pb}$ concentrations. However, it should be noted that all $\mathrm{Pb}$ concentrations after neutralisation were close to detection levels. 
HS - Metal Removal

The average raw AMD concentration for the relevant metals over the experimental period is shown on Figure 7-1.

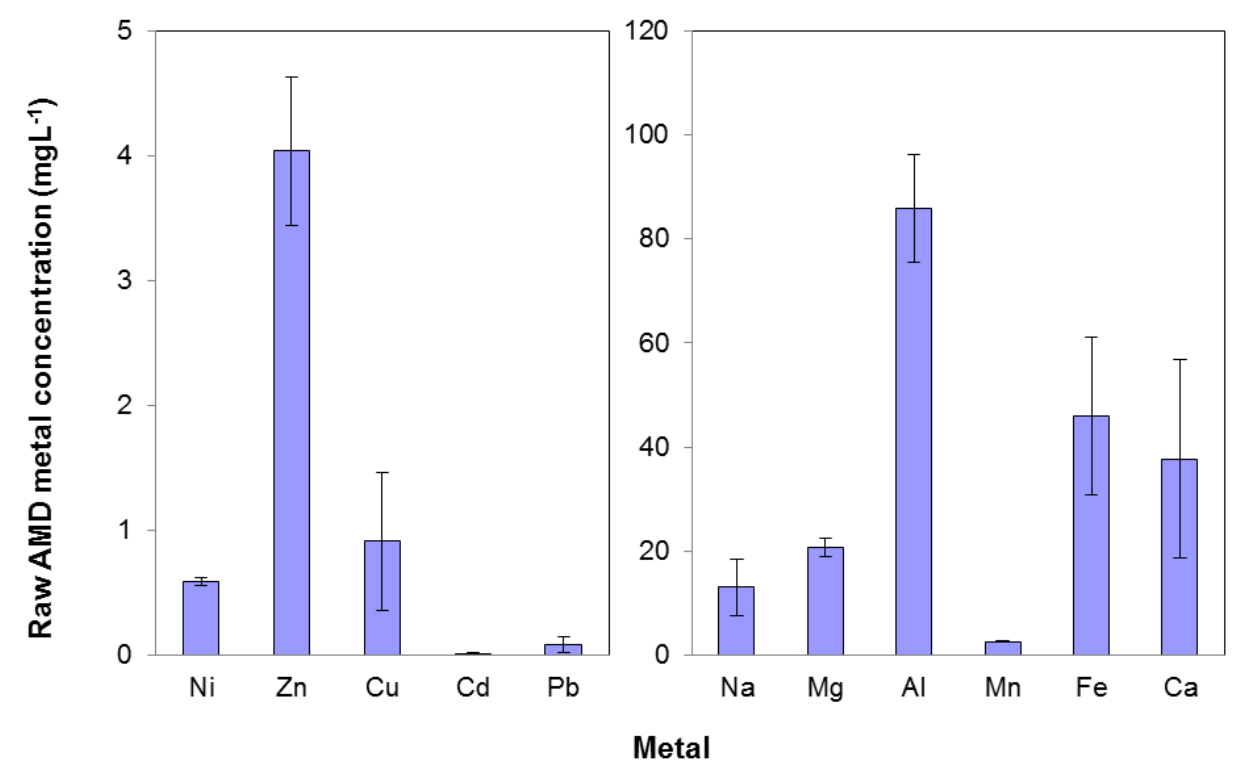

Figure 7-1: Raw AMD concentration. Standard deviation error ( $n=15)$

The left section of the figure shows the average raw concentration of the target metal group ( $\mathrm{Ni}, \mathrm{Zn}, \mathrm{Cu}, \mathrm{Cd}$ and $\mathrm{Pb}$ ). The concentration of $\mathrm{Zn}$ is greatest at $4.0 \mathrm{mg} / \mathrm{L}$ followed by $\mathrm{Cu}$ and $\mathrm{Ni}$ at 0.9 and $0.6 \mathrm{mg} / \mathrm{L}$, respectively. The concentration of $\mathrm{Cd}$ and $\mathrm{Pb}$ was 0.01 and $0.06 \mathrm{mg} / \mathrm{L}$.

The right section shows the concentration of other important cations. The concentration of $\mathrm{Al}$ and Fe were significantly elevated at 86.0 and $45.9 \mathrm{mg} / \mathrm{L}$, respectively. The other cations $(\mathrm{Na}, \mathrm{Mg}, \mathrm{Mn}$, and $\mathrm{Ca}$ ) were measured as they are important for the equilibrium speciation modelling. However, the concentrations of these cations were not discussed with respect to metal removal.

Instrument detection limits were determined by the three sigma method, discussed in section 3.3.1.6. The instrument detection limits for the target metal group are shown in Table 7-1. 
HS - Metal Removal

Table 7-1: Thesis metal detection limits

\begin{tabular}{ccc}
\hline Metal & $\begin{array}{c}\text { Instrument Detection Limit } \\
(\mathbf{u g} / \mathbf{L})\end{array}$ & $\begin{array}{c}\text { Experimental Detection Limit } \\
(\mathbf{m g} / \mathbf{L})\end{array}$ \\
\hline $\mathbf{N i}$ & 0.067 & 0.007 \\
$\mathbf{Z n}$ & 0.143 & 0.027 \\
$\mathbf{C u}$ & 0.272 & 0.014 \\
$\mathbf{C d}$ & 0.011 & 0.001 \\
$\mathbf{P b}$ & 0.244 & 0.024 \\
\hline
\end{tabular}

The "experimental detection limit" is the detection limit used in discussion throughout this thesis. It was determined as 100 times the instrument detection limit, to account for sample dilution (100x). All raw AMD concentrations were greater than the detection limit. The raw concentration of $\mathrm{Pb}$ was twice the detection limit, while the rest of the target metal suite were significantly above their respective detection limits. 
HS - Metal Removal

\subsection{Metal Removal}

This section discusses the results of metal analysis from the HS jar testing supernatants. The results of control standards are discussed first. The effect of HS addition is then discussed based on the HS dosed metal concentrations.

Excessively high concentrations of $\mathrm{Al}$ and Fe were identified in the raw AMD in section 7.1. After neutralisation the concentration of $\mathrm{Al}$ and $\mathrm{Fe}$ was at or below detection levels. Thus, $\mathrm{Al}$ and $\mathrm{Fe}$ are not discussed beyond this point.

The $\mathrm{pH}$ of jar testing was difficult to control due to humic buffering and the inaccuracies of dosing with supersaturated slurry. As a result the $\mathrm{pH}$ of different HS dose conditions varied. Therefore the metal removal could not be directly compared between different HS conditions. The HS dosed metal concentrations were plotted beside a control metal concentration obtained by alkaline neutralisation only with the same $\mathrm{pH}$ as the HS dosed condition.

All discussion on HS enhanced metal removal is with reference to the control metal concentration, not the raw AMD concentration. Thus, a quoted 25\% increase in metal removal by HS addition corresponds to $25 \%$ lower residual metal concentration in the HS dosed sample compared to the control sample, at the same $\mathrm{pH}$. The neutralisation dose volumes of $16.3,8.45$ and $18.0 \mathrm{~mL}$ for $\mathrm{NaOH}, \mathrm{Ca}(\mathrm{OH})_{2}$ and $\mathrm{CaCO}_{3}$ were deemed to have a negligible dilution effect $(<1.8 \%)$ on metal concentration. 


\subsubsection{BTP Metal Removal}

This section discusses the results of the Blackwater Treatment Plant (BTP) HS jar testing samples. The results for $\mathrm{NaOH}$ are discussed first, followed by $\mathrm{Ca}(\mathrm{OH})_{2}$. The HS enhanced metal removal in this section was compared to a control standard at the same $\mathrm{pH}$ for each condition. Many of these conditions had HS dosed and control standard error bars which overlapped. Conclusive correlations between HS addition and metal removal for these data cannot be made; although trends in these data were identified where appropriate.

The supernatant metal concentrations during the sedimentation period for the BTP trials were found to be independent of time, due to rapid neutralisation of $\mathrm{NaOH}$ and $\mathrm{Ca}(\mathrm{OH})_{2}$. The BTP results were therefore presented as the average metal concentrations for the eight samples collected over the 2 minute to 2 hour sampling period, for the given HS dosing scenario. This observation implies that the dominant metal removal mechanisms (precipitation, adsorption, etc.) operate relatively instantaneously also. 
HS - Metal Removal

\subsubsection{NaOH Metal Removal}

\subsection{NaOH Control standards}

The metal concentrations for the control samples neutralised by $\mathrm{NaOH}$ to between $\mathrm{pH} 6$ and 7 were plotted against pH on Figure 7-2. Raw data are included in Appendix 5.
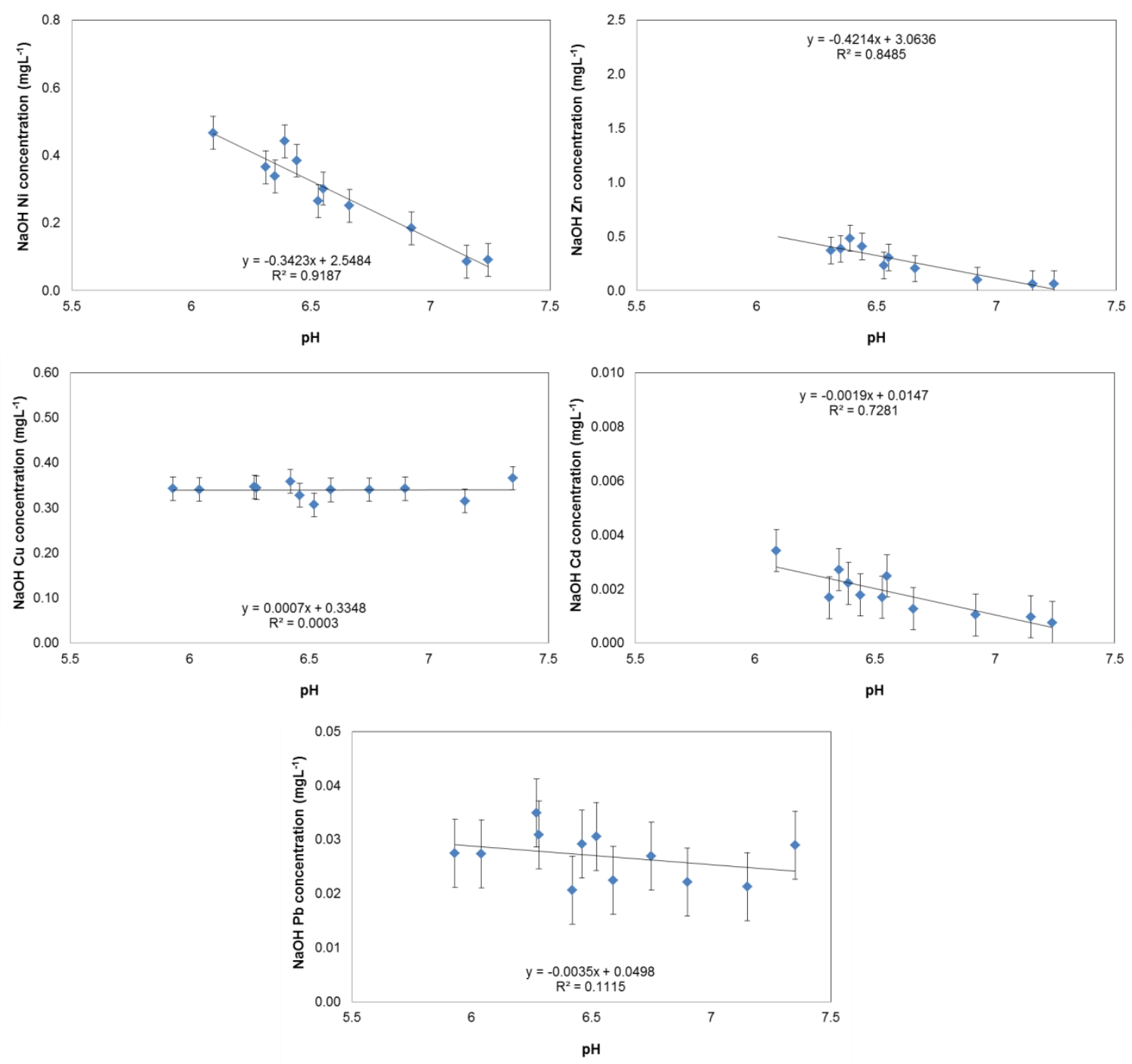

Figure 7-2: NaOH neutralised control standards. Standard deviation error (n=11).

The data plotted on Figure 7-2 were fitted with a linear trendline for $\mathrm{Ni}, \mathrm{Zn}$ and $\mathrm{Cd}$, all with relatively high $\mathrm{R}^{2}$ values. The $\mathrm{Ca}(\mathrm{OH})_{2}$ control standard data for $\mathrm{Cu}$ and $\mathrm{Pb}$ was used instead of the $\mathrm{NaOH}$ data due to inexplicably low concentrations for $\mathrm{Cu}$ and $\mathrm{Pb}$ (analytical errors) in the $\mathrm{NaOH}$ case. The equations of the trend lines and error are shown in Table 7-2. 
HS - Metal Removal

Table 7-2: NaOH neutralised control standard trend lines and residuals

\begin{tabular}{ccc}
\hline Metal & Trendline & error \\
\hline $\mathbf{N i}$ & $\mathrm{y}=-0.3423 \mathrm{x}+2.5484$ & 0.049 \\
$\mathbf{Z n}$ & $\mathrm{y}=-0.4214 \mathrm{x}+3.0636$ & 0.112 \\
$\mathbf{C u}$ & $\mathrm{y}=0.0007 \mathrm{x}+0.3348$ & 0.026 \\
$\mathbf{C d}$ & $\mathrm{y}=-0.0019 \mathrm{x}+0.0147$ & 0.001 \\
$\mathbf{P b}$ & $\mathrm{y}=-0.0035 \mathrm{x}+0.0498$ & 0.006 \\
\hline
\end{tabular}

The error values in Table 7-2 were obtained by calculating the residuals for each metal, using

$$
e=y-\hat{y}
$$

Equation 7-1

Residual equation

Where, e is the residual;

$\mathrm{y}$ is the measured metal concentration, at a specific $\mathrm{pH}$; and

$\hat{y}$ is the theoretical control metal concentration for the same $\mathrm{pH}$.

The $90^{\text {th }}$ percentile was then calculated over the 12 residuals for the control standards and used as the error for the control standards. The trendline equations were used to calculate the control standard metal concentrations at the specific $\mathrm{pH}$ of the corresponding $\mathrm{HS}$ dosed sample.

The removal of $\mathrm{Ni}, \mathrm{Zn}$ and $\mathrm{Cd}$ by $\mathrm{NaOH}$ neutralisation from control samples was highly dependent on $\mathrm{pH}$. The concentration of $\mathrm{Ni}$ decreased linearly from the raw AMD concentration by $16 \%$ at $\mathrm{pH} 6$ to $74 \%$ at $\mathrm{pH} 7$. Hydroxide precipitation of $\mathrm{Ni}$ at $\mathrm{pH} 7$ would be negligible (Kelly 1988). Removal of Ni by co-precipitation is unlikely as the removal of $\mathrm{Ni}$ was low at $\mathrm{pH} \mathrm{6}$, after precipitation of $\mathrm{Fe}(\mathrm{pH} 3.5)$ and $\mathrm{Al}(\mathrm{pH}$ 5) had occurred. Thus Ni removal must have occurred by adsorption when hydroxide precipitates become negatively charged at neutral pH (Sparks 2003). The inferred adsorption of Ni to metal hydroxide precipitates would be very similar to that shown in the literature, at pH 7 (Lee, Bigham et al. 2002; Xu, Axe et al. 2007).

The concentration of $\mathrm{Zn}$ decreased from the raw AMD concentration by $70 \%$ at $\mathrm{pH} 6$ to $98 \%$ at $\mathrm{pH}$ 7. A small amount of hydroxide precipitation of $\mathrm{Zn}$ may have occurred, as 
rapid precipitation of $\mathrm{Zn}$ occurs at just over $\mathrm{pH} 8$ (Kelly 1988). However, adsorption is probably the dominant $\mathrm{Zn}$ removal mechanism. The adsorption of $\mathrm{Zn}$ to metal hydroxides has been shown to occur between pH 5 and 6 in the literature (Webster, Swedlund et al. 1998; Lee, Bigham et al. 2002; Davies, Weber et al. 2011a). Therefore, rapid removal of $\mathrm{Zn}$ by adsorption probably occurred at $\mathrm{pH}$ less than 6 , just outside the range investigated.

The concentration of Cd decreased from the raw AMD concentration by $71 \%$ at $\mathrm{pH} 6$ to $88 \%$ at $\mathrm{pH}$ 7. Hydroxide precipitation of $\mathrm{Cd}$ is unlikely at $\mathrm{pH} 7$ (Kelly 1988). The continued decrease in $\mathrm{Cd}$ concentration with increasing $\mathrm{pH}$ (beyond $\mathrm{pH}$ 6) shows that adsorption is an important $\mathrm{Cd}$ removal mechanism. Cadmium removal by co-precipitation and adsorption is supported by the literature (Webster, Swedlund et al. 1998; Lee, Bigham et al. 2002). However, based on the data available it is not possible to determine the ratio of $\mathrm{Cd}$ removed by each mechanism.

The trends shown by $\mathrm{Cu}$ and $\mathrm{Pb}$ are discussed in the $\mathrm{Ca}(\mathrm{OH})_{2}$ control standards section (section 7.2.1.2.1). 


\subsection{NaOH Metal Removal}

Figure 7-3 shows the metal concentrations of the $\mathrm{NaOH}$ HS trials. Raw data are included in Appendix 6.
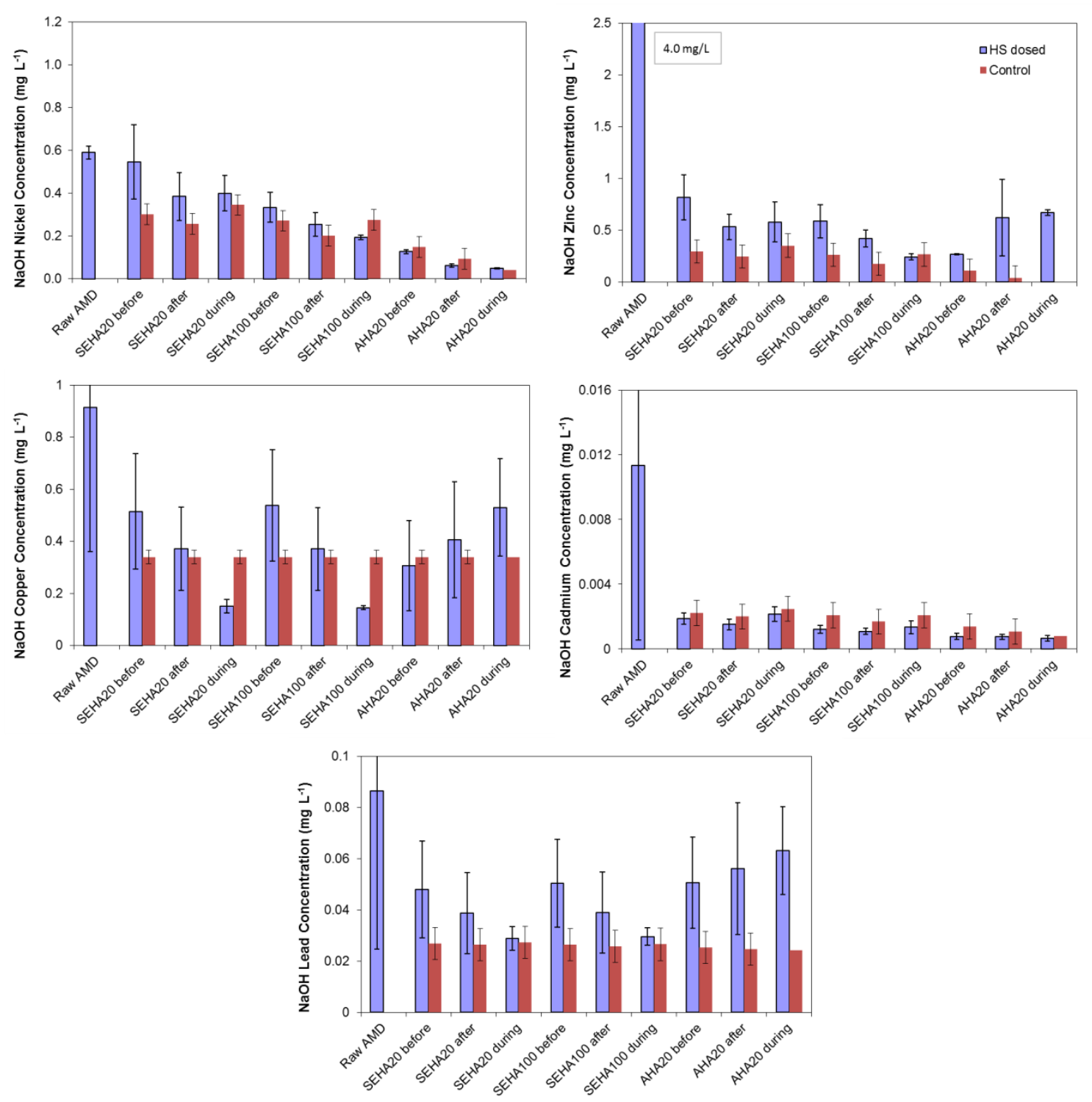

Figure 7-3: NaOH HS dosed metal concentrations. Standard deviation error ( $n \geq 11)$.

The results for $\mathrm{Ni}$ and $\mathrm{Zn}$ show no real HS removal enhancement trends. The HS amended $\mathrm{Ni}$ and $\mathrm{Zn}$ concentrations were typically higher than their respective control concentrations. The complexation of $\mathrm{Ni}$ and $\mathrm{Zn}$ was expected to be low, based on the literature (Milne, Kinniburgh et al. 2003). A 100 mg/L humic acid (HA) dose would have the capacity to bind 0.13 and $0.013 \mathrm{mg}$ of $\mathrm{Zn}$ and $\mathrm{Ni}$, respectively (Milne, Kinniburgh et al. 2003). Thus, an HS dose of even $100 \mathrm{mg} / \mathrm{L}$ may have been too low to observe any effect on $\mathrm{Ni}$. 
Enhancement of $\mathrm{Cu}$ removal by HS was dependent on dose order. The SEHA20 and SEHA100 during-neutralisation conditions enhanced $\mathrm{Cu}$ removal by 55 and $57 \%$, respectively. The additional removal of approximately $0.2 \mathrm{mg} \mathrm{Cu} / \mathrm{L}$ by addition of 20 $\mathrm{mg} / \mathrm{L}$ Solid Energy Humic Acid (SEHA) results in a potential removal capacity of 10 $\mathrm{mg} / \mathrm{g}$ for $\mathrm{Cu}$, which is similar to the $25 \mathrm{mg} / \mathrm{g}$ value obtained from Milne et al. (2003). Addition of $\mathrm{HS}$ after neutralisation may have enhanced $\mathrm{Cu}$ removal but the magnitude of the error made these data unreliable. Enhanced removal of $\mathrm{Cu}$ by $\mathrm{HS}$ addition was attributed to the fact that $\mathrm{Cu}$ has a relatively high binding affinity for HS and is relatively high on the complexation hierarchy (Milne, Kinniburgh et al. 2003). The data for AHA do not compliment the SEHA data, showing AHA20 before-neutralisation resulting in the lowest $\mathrm{Cu}$ concentration; the reason for the discrepancy of AHA results is uncertain.

The data for Cd suggest all HS dose conditions resulted in a small increase in Cd removal. This observation has yet to be confirmed as the error bars of the HS dosed and control samples overlap and some data were close to the Cd detection limit $(0.001 \mathrm{mg} / \mathrm{L})$. The SEHA100 conditions resulted in lower Cd concentrations than the SEHA20 conditions, suggesting Cd removal is positively correlated to HS dose. The complexation capacity of $\mathrm{HS}$ for $\mathrm{Cd}$ is relatively low, however $\mathrm{Cd}$ concentrations were typically very low, resulting in a relatively high percentage of Cd complexation. A $100 \mathrm{mg} / \mathrm{L} \mathrm{HA}$ dose could theoretically complex $0.06 \mathrm{mg} \mathrm{Cd}$ (Milne, Kinniburgh et al. 2003), which is significantly higher than even the raw AMD Cd concentration.

The data for $\mathrm{Pb}$ showed that no $\mathrm{HS}$ conditions enhanced $\mathrm{Pb}$ removal. The lowest $\mathrm{HS}$ dosed $\mathrm{Pb}$ concentrations occurred for the SEHA20 and SEHA100 during-neutralisation conditions. For this condition the $\mathrm{Pb}$ concentration was approximately equal to the control. Similarly to $\mathrm{Cd}, \mathrm{Pb}$ data were indeterminate due to overlapping error bars and concentrations approaching the detection limit of $0.024 \mathrm{mg} / \mathrm{L}$.

In theory, a greater removal of $\mathrm{Pb}$ was expected as $\mathrm{Pb}$ has a high binding affinity for $\mathrm{HS}$ of up to $26 \mathrm{mg} / \mathrm{g}$ (Milne, Kinniburgh et al. 2003). The adsorption capacity of $\mathrm{Pb}$ to iron hydroxide precipitates is also very high (Lee, Bigham et al. 2002). Due to the relatively low $\mathrm{Pb}$ concentration in the raw $\mathrm{AMD}$, complete removal of $\mathrm{Pb}$ to below detection levels is probable by either HS complexation or hydroxide adsorption. The relatively high experimental detection limit for $\mathrm{Pb}$ however made these trends difficult to identify. 
Section 6.2.1.1 showed the addition of HS after neutralisation by $\mathrm{NaOH}$ resulted in significant turbidity and suspended solids (SS) in the neutralised samples. A lack of multi-valent flocculating cations was suggested as the reason for HS material remaining dissolved and not settling during the sedimentation period. The addition of HS resulted in higher concentrations of $\mathrm{Ni}, \mathrm{Zn}, \mathrm{Cu}$ and $\mathrm{Pb}$ for some conditions. The diameter of $\mathrm{HS}$ range from 1 to $0.001 \mu \mathrm{m}$ (Sparks 2003), thus some HS complexes may pass through a $0.45 \mu \mathrm{m}$ filter, possibly resulting in higher metal concentrations than the control where adsorbed/precipitated metals were filtered out. These phenomena would explain the higher metal concentration in HS dosed scenarios if high metal concentrations were observed, particularly for the SEHA100 after-neutralisation condition which resulted in very high turbidity/SS and was significantly discoloured. However, the metal concentrations of SEHA 100 after-neutralisation conditions were in no way exceptional, suggesting that most of the HS complexes formed at high HS concentrations were excluded by the $0.45 \mu \mathrm{m}$ filter. The elevated metal concentrations may therefore be the result of experimental and analytical variability.

The incorporation of $\mathrm{HS}$ in AMD neutralisation by $\mathrm{NaOH}$ does not widely enhance metal removal. The removal of $\mathrm{Cd}$ under all HS dosed conditions appears to be enhanced by HS, while HS addition during neutralisation resulted in significant additional removal of $\mathrm{Cu}$. 
HS - Metal Removal

\subsubsection{2 $\mathrm{Ca}(\mathrm{OH})_{2}$ Metal Removal}

\subsubsection{1 $\mathrm{Ca}(\mathrm{OH})_{2}$ Control standards}

The metal concentrations for the control samples neutralised by $\mathrm{Ca}(\mathrm{OH})_{2}$ to between $\mathrm{pH} 6$ and 7 were plotted against pH on Figure 7-4. Raw data are included in Appendix 5.
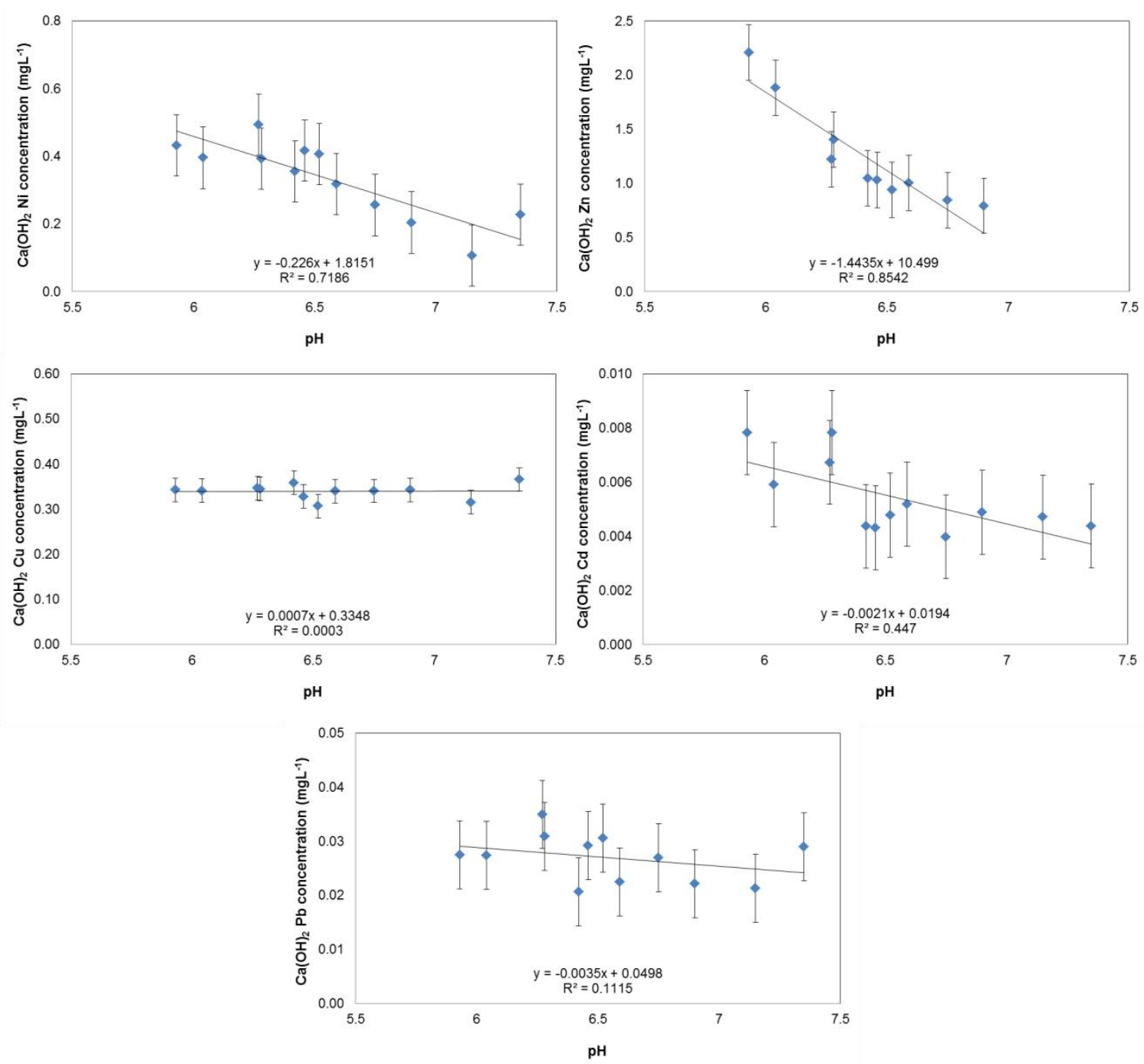

Figure 7-4: $\mathrm{Ca}(\mathrm{OH})_{2}$ standard control Standard deviation error $(n=11)$.

The data plotted on Figure 7-4 were fitted with a linear trendline with good $\mathrm{R}^{2}$ values for $\mathrm{Ni}, \mathrm{Zn}$ and $\mathrm{Cd}$ between 0.447 and 0.8542 . The $\mathrm{Cu}$ and $\mathrm{Pb}$ concentration was poorly correlated with $\mathrm{pH}$, as shown by $\mathrm{R}^{2}$ values of 0.0003 and 0.1115 , respectively. The equations of trend lines and error are shown in Table 7-3. 
HS - Metal Removal

Table 7-3: $\mathrm{Ca}(\mathrm{OH})_{2}$ neutralised control standard trend lines and error

\begin{tabular}{ccc}
\hline Metal & Trend line & Error \\
\hline $\mathbf{N i}$ & $\mathrm{y}=-0.226 \mathrm{x}+1.8151$ & 0.091 \\
$\mathbf{Z n}$ & $\mathrm{y}=-1.4435 \mathrm{x}+10.499$ & 0.255 \\
$\mathbf{C u}$ & $\mathrm{y}=0.0007 \mathrm{x}+0.3348$ & 0.026 \\
$\mathbf{C d}$ & $\mathrm{y}=-0.0021 \mathrm{x}+0.0194$ & 0.002 \\
$\mathbf{P b}$ & $\mathrm{y}=-0.0035 \mathrm{x}+0.0498$ & 0.006 \\
\hline
\end{tabular}

The removal of $\mathrm{Ni}$ by $\mathrm{Ca}(\mathrm{OH})_{2}$ neutralisation was dependent on $\mathrm{pH}$. The concentration of $\mathrm{Ni}$ decreased from the raw AMD concentration by $22 \%$ at $\mathrm{pH} 6$ to $61 \%$ at $\mathrm{pH} 7$. The removal of $\mathrm{Zn}$ was also dependent on $\mathrm{pH}$, with a linear decrease in concentration from raw $\mathrm{AMD}$ by $55 \%$ at $\mathrm{pH} 6$ to $85 \%$ at $\mathrm{pH}$. The dominant mechanism for $\mathrm{Ni}$ and $\mathrm{Zn}$ removal was probably adsorption to metal hydroxide precipitates, as discussed in section 7.2.1.1.1.

The removal of $\mathrm{Cu}$ by $\mathrm{Ca}(\mathrm{OH})_{2}$ neutralisation was independent of $\mathrm{pH}$ over the range investigated. A $\mathrm{Cu}$ concentration of $0.34 \mathrm{mg} / \mathrm{L}$ was measured at both $\mathrm{pH} 6$ and 7 . Hydroxide precipitation of $\mathrm{Cu}$ was expected to occur between $\mathrm{pH} 6.8$ and 7.2, however the constant $\mathrm{Cu}$ concentration does not suggest hydroxide precipitation or adsorption occurred. At pH 6-7, the raw AMD Cu concentration had already been reduced by $63 \%$. Lee et al. (2002) showed almost $100 \%$ of $\mathrm{Cu}$ adsorption occurred simultaneously with $\mathrm{Al}$ hydroxide precipitation, over the $\mathrm{pH}$ range 4.5 to 5 , suggesting $\mathrm{Cu}$ removal by coprecipitation with $\mathrm{Al}$ hydroxide.

The removal of $\mathrm{Cd}$ was dependent on $\mathrm{pH}$, with a linear decrease in concentration from raw AMD by $40 \%$ at $\mathrm{pH} 6$ to $59 \%$ at $\mathrm{pH} 7$. The slope of the $\mathrm{Cd}$ trendline was similar to $\mathrm{NaOH}$ neutralised AMD, suggesting that metal removal was by adsorption, as discussed in section 7.2.1.1.1.

The concentration of $\mathrm{Pb}$ was at the detection limit. Lee et al. (2002) showed that $\mathrm{Pb}$ adsorption was strongly correlated to precipitation of Fe during AMD neutralisation. This suggests $\mathrm{Pb}$ was removed by co-precipitation to $\mathrm{Fe}(\mathrm{OH})_{3}$, although $\mathrm{Pb}$ may also coprecipitate with $\mathrm{Al}$ hydroxides. Hydroxide precipitation of $\mathrm{Pb}$ below $\mathrm{pH} 6$ may have also 
HS - Metal Removal

reduced $\mathrm{Pb}$ concentration (Kelly 1988). Thus, control neutralisation of AMD would result in $\mathrm{Pb}$ concentrations below the detection limit.

Overall, the removal of $\mathrm{Ni}, \mathrm{Zn}$ and $\mathrm{Cd}$ was lower when neutralised by $\mathrm{Ca}(\mathrm{OH})_{2}$ compared to $\mathrm{NaOH}$. Neutralisation by $\mathrm{Ca}(\mathrm{OH})_{2}$ resulted in Ca concentrations of approximately 400 $\mathrm{mg} / \mathrm{L}$. As Ca is divalent, it has a greater polarisation potential than $\mathrm{Na}$ (Evangelou 1998) and therefore a greater affinity for adsorption. Thus, Ca competition for adsorption sites on hydroxide precipitates may have displaced adsorbed metal cations, resulting in greater supernatant concentrations. 


\subsubsection{2 $\mathrm{Ca}(\mathrm{OH})_{2}$ Metal Removal}

Figure 7-5 shows the metal concentrations of the $\mathrm{Ca}(\mathrm{OH})_{2}$ HS trials. Raw data are included in Appendix 6.
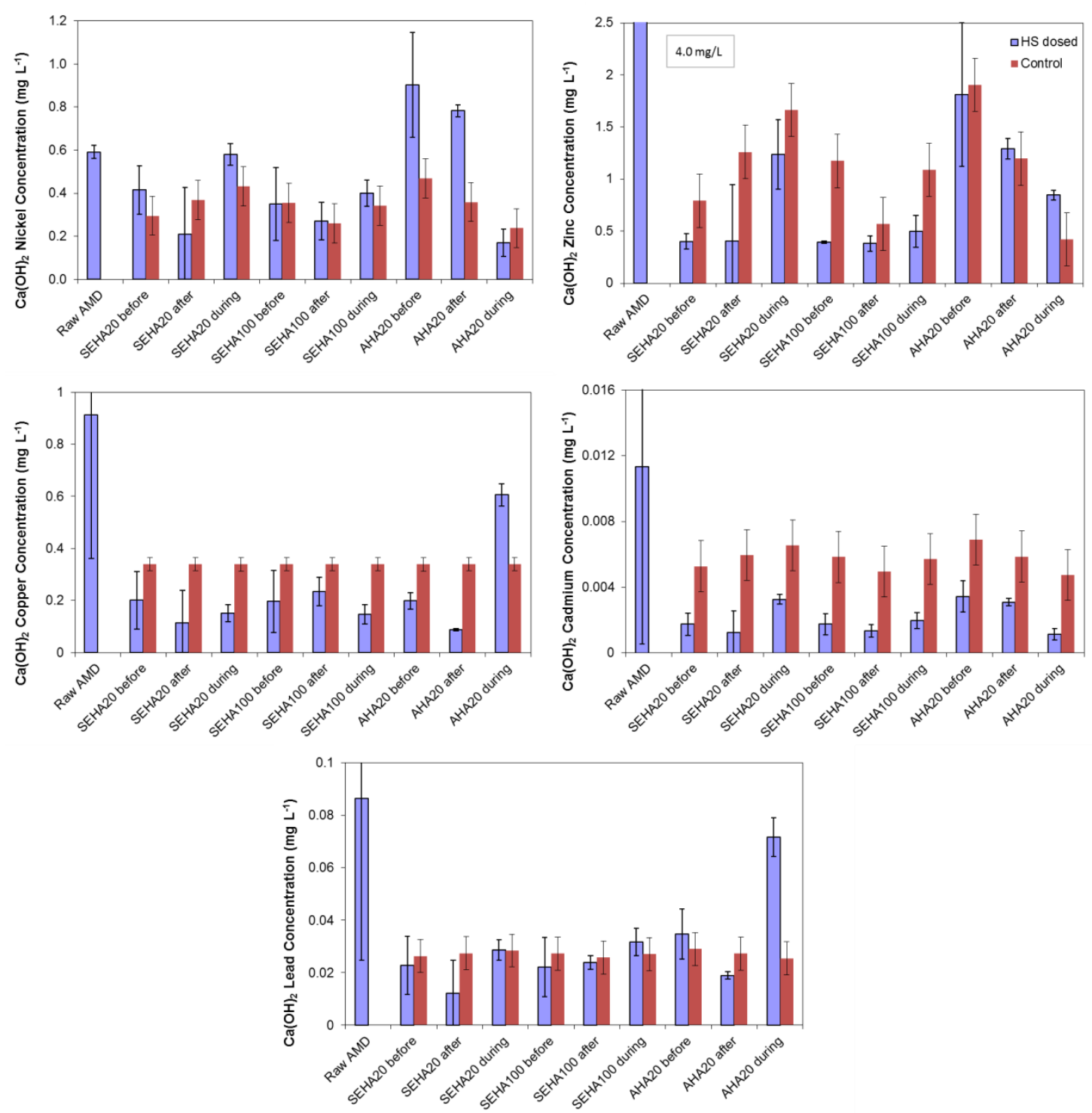

Figure 7-5: Metal concentrations of $\mathrm{Ca}(\mathrm{OH})_{2}$ neutralised AMD. Standard deviation error $(n \geq 11)$.

Figure 7-5 shows $\mathrm{HS}$ does not significantly enhance $\mathrm{Ni}$ removal during $\mathrm{Ca}(\mathrm{OH})_{2}$ neutralisation, probably due to the low complexation affinity of Ni for HS. The HS dosed conditions typically have a higher Ni concentration than their respective control. NB: the Ni contributed by SEHA was minimal, as discussed in section 7.2.2.1.2. The SEHA20 after-neutralisation condition appears to enhance Ni removal but the error bars overlap the control, making these data unreliable. 
Figure 7-5 shows the addition of SEHA generally enhanced the removal of $\mathrm{Zn}$ during $\mathrm{Ca}(\mathrm{OH})_{2}$ neutralisation of AMD. Milne et al. (2003) determined the complexation capacity of HS for $\mathrm{Zn}$ was $1.3 \mathrm{mg} / \mathrm{g}$. The $20 \mathrm{mg} / \mathrm{L}$ HS dosed scenarios removed up to 0.6 $\mathrm{mg} \mathrm{Zn}$ for a HS dose of $0.02 \mathrm{~g}$, which corresponds to a complexation capacity 20 times greater than that reported by Milne et al. (2003).

The elevated $\mathrm{Ca}$ concentration (due to $\mathrm{Ca}(\mathrm{OH})_{2}$ dissolution) should have reduced the $\mathrm{Zn}$ removal by competitive adsorption to hydroxide precipitates. Although adsorption of HS complexed $\mathrm{Zn}$ to hydroxide precipitates may have resulted in additional $\mathrm{Zn}$ removal, Warren et al. (1994) found the addition of HS did not enhance the sorption of $\mathrm{Zn}$ onto Fe and Mn oxides.

An alternative explanation for what appears to be HS enhanced removal of $\mathrm{Zn}$ may be experimental error. The $\mathrm{Ca}(\mathrm{OH})_{2}$ neutralised control standard samples had a greater residual $\mathrm{Zn}$ concentration $(0.6-1.8 \mathrm{mg} / \mathrm{L})$ than the $\mathrm{NaOH}$ neutralised control conditions (0.1-1.2 mg/L). The HS dosed conditions neutralised by $\mathrm{NaOH}(0.5 \mathrm{mg} / \mathrm{L})$ and $\mathrm{Ca}(\mathrm{OH})_{2}$ (0.4-1.2 $\mathrm{mg} / \mathrm{L})$ had similar residual $\mathrm{Zn}$ concentrations to the $\mathrm{NaOH}$ control. Thus, the elevated $\mathrm{Ca}(\mathrm{OH})_{2}$ neutralised control $\mathrm{Zn}$ concentrations (compared to $\mathrm{NaOH}$ neutralised control concentrations) made the addition of HS appear to significantly reduce the $\mathrm{Zn}$ concentration.

Removal of $\mathrm{Cu}$ was almost uniformly enhanced by HS addition, with the AHA20 duringneutralisation condition the only exception. The AHA20 and SEHA20 after-neutralisation conditions enhanced $\mathrm{Cu}$ removal by 74 and $66 \%$ over the control. An increase in HS dose from 20 to $100 \mathrm{mg} / \mathrm{L}$ resulted in a $2 \%$ increase in $\mathrm{Cu}$ removal for both before- and duringneutralisation conditions, suggesting the $20 \mathrm{mg} / \mathrm{L}$ HS dose was sufficient to complex all $\mathrm{Cu}$. According to Milne et al. (2003), a $20 \mathrm{mg} / \mathrm{L} \mathrm{HS}$ dose should have the capacity to complex $0.5 \mathrm{mg} \mathrm{Cu}$, which would theoretically be sufficient to remove all residual $\mathrm{Cu}$ from control neutralised AMD. Warren et al. (1994) showed that addition of HS enhanced oxide adsorption of $\mathrm{Cu}$, probably due to adsorption of HS-Cu complexes. Thus, removal of $\mathrm{Cu}$ during sedimentation was enhanced by HS complexation and subsequent adsorption to settling insoluble hydroxides.

The addition of HS enhanced removal of Cd under all HS dose conditions. Addition of SEHA 20 and 100 after neutralisation resulted in additional Cd removal of 79 and $72 \%$, suggesting the $20 \mathrm{mg} / \mathrm{L}$ HS dose was sufficient for Cd complexation. The $20 \mathrm{mg} / \mathrm{L} \mathrm{HS}$ 
dose should have the capacity to complex $0.012 \mathrm{mg} \mathrm{Cd}$ (Milne, Kinniburgh et al. 2003), exceeding the amount of residual $\mathrm{Cd}$ in the solution. The control neutralised $\mathrm{Cd}$ concentration was greater for $\mathrm{Ca}(\mathrm{OH})_{2}$ neutralisation, therefore the final Cd concentration for the SEHA20 after-neutralisation condition was still above detection limits after 79\% removal.

The data for both control and HS dosed $\mathrm{Pb}$ samples were at the detection limit of 0.024 $\mathrm{mg} / \mathrm{L}$. Similarly to the $\mathrm{NaOH}$ trials, $\mathrm{Pb}$ was removed to detection limits due to its high $\mathrm{HS}$ binding capacity (Milne, Kinniburgh et al. 2003) and strong adsorption capacity to iron hydroxide precipitates (Lee, Bigham et al. 2002).

Overall, HS dosing had a greater impact on metal removal in $\mathrm{Ca}(\mathrm{OH})_{2}$ neutralised conditions. Neutralisation by $\mathrm{Ca}(\mathrm{OH})_{2}$ resulted in greater residual metal concentrations, probably due to competition by $\mathrm{Ca}$ for adsorption sites on hydroxide precipitates. The greater residual metal concentrations made HS enhanced metal removal more noticeable. Addition of SHEA before neutralisation resulted in good removal of $\mathrm{Zn}$ and Cd. Addition of SEHA and AHA after neutralisation resulted in good removal of $\mathrm{Zn}, \mathrm{Cu}$ and $\mathrm{Cd}$. Addition of SEHA during neutralisation resulted in good removal of $\mathrm{Cu}$. In general, addition of HS after neutralisation resulted in the greatest metal removal, suggesting that preventing binding competition from $\mathrm{Fe}$ and $\mathrm{Al}$ is important in the optimisation of HS enhanced neutralisation of AMD.

Table 7-4 shows the corresponding ANZECC level of protection for the residual BTP metal concentrations.

Table 7-4: BTP level of protection of treated AMD

\begin{tabular}{ccccc}
\hline & \multicolumn{2}{c}{$\mathbf{N a O H}^{*}$} & \multicolumn{2}{c}{$\mathbf{C a}(\mathbf{O H})_{2}{ }^{* *}$} \\
& Control & HS dosed & Control & HS dosed \\
\hline $\mathbf{N i}$ & $<80$ & $<80$ & 99 & 99 \\
$\mathbf{Z n}$ & $<80$ & $<80$ & 80 & 80 \\
$\mathbf{C u}$ & - & $<80$ & $<80$ & $<80$ \\
$\mathbf{C d}$ & 90 & 90 & 90 & 99 \\
$\mathbf{P b}$ & - & 90 & 95 & 99 \\
\hline$* 210 \mathrm{mg} / \mathrm{L} \mathrm{CaCO}_{3}$ hardness & & & \\
$*$ & & &
\end{tabular}


Table 7-4 shows that while some $\mathrm{NaOH}$ neutralised HS dosed conditions resulted in lower metal concentrations than control standards, the improvement was insufficient to significantly improve the level of protection to aquatic species. The concentration of $\mathrm{Ni}$, $\mathrm{Zn}$ and $\mathrm{Cu}$ after treatment exceeded the recommended limit for protection of $80 \%$ of species. The toxicity of $\mathrm{NaOH}$ neutralised AMD was relatively high due to the low hardness $\left(\sim 210 \mathrm{mg} / \mathrm{L} \mathrm{CaCO}_{3}\right)$ resulting in minimal detoxification of metals.

The level of protection for $\mathrm{Cd}$ and $\mathrm{Pb}$ were improved by $\mathrm{HS}$ dosing in the $\mathrm{Ca}(\mathrm{OH})_{2}$ neutralised BTP trials. The SEHA20 after-, SEHA100 and AHA20 during-neutralisation conditions resulted in sufficient additional $\mathrm{Cd}$ removal to raise the level of protection from $90 \%$ to $99 \%$. The SEHA20 after-neutralisation condition also increased the level of $\mathrm{Pb}$ protection from 95 to $99 \%$ of species. The high hardness of $\mathrm{Ca}(\mathrm{OH})_{2}$ neutralised AMD $\left(1000 \mathrm{mg} / \mathrm{L} \mathrm{CaCO}_{3}\right)$, resulted in a considerably higher level of protection (except for $\mathrm{Cu}$ ) compared to $\mathrm{NaOH}$ neutralised AMD. 
HS - Metal Removal

\subsubsection{MSS Metal Removal}

The metal concentrations for the Mangatini Stream-sump System (MSS) trials were time dependent, as continued $\mathrm{pH}$ increase occurred due to continued dissolution of residual $\mathrm{CaCO}_{3}$. The MSS results are therefore presented as kinetic metal concentrations for the six (2, 6, 24 hrs and 3, 5, 7 day) sampling times during the sedimentation period.

\subsubsection{1 $\mathrm{CaCO}_{3}$ Metal Removal}

\subsubsection{1 $\mathrm{CaCO}_{3}$ Control Standards}

The metal concentrations for the control samples neutralised by $\mathrm{CaCO}_{3}$ to between $\mathrm{pH} 6$ and 8 were plotted against $\mathrm{pH}$, as shown on Figure 7-6. Raw data are included in Appendix 5.
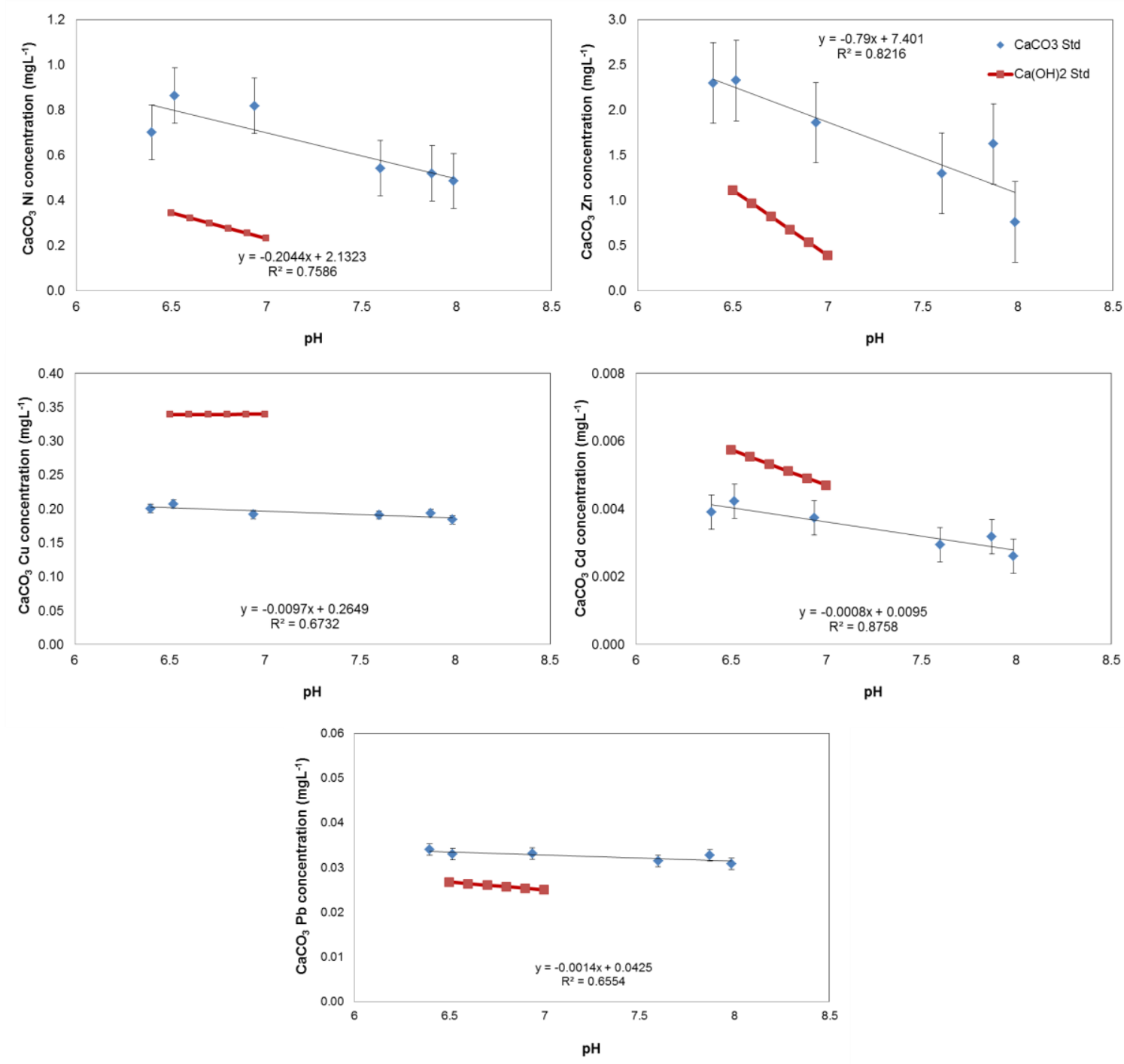

Figure 7-6: $\mathrm{CaCO}_{3}$ control standards . Standard deviation error ( $\left.n=6\right)$. 
HS - Metal Removal

The $\mathrm{CaCO}_{3}$ control standards had much higher $\mathrm{R}^{2}$ values than BTP conditions (possibly due to fewer data points), ranging from 0.6554 to 0.8758 . The second series on Figure 7-6 shows the $\mathrm{Ca}(\mathrm{OH})_{2}$ standard concentrations for comparison, although the $\mathrm{Ca}(\mathrm{OH})_{2}$ models were not extrapolated beyond $\mathrm{pH}$ 7. The residual $\mathrm{Ni}, \mathrm{Zn}$ and $\mathrm{Pb}$ concentration was considerably higher between $\mathrm{pH} 6.5$ and 7 using $\mathrm{CaCO}_{3}$ for neutralisation. The removal of $\mathrm{Cu}$ and $\mathrm{Cd}$ however was greater using $\mathrm{CaCO}_{3}$. The equations of trend lines and error are shown in Table 7-5.

Table 7-5: $\mathrm{Ca}(\mathrm{OH})_{3}$ neutralised control standard trend lines and error

\begin{tabular}{ccc}
\hline Metal & Trend line & Error \\
\hline $\mathbf{N i}$ & $\mathrm{y}=-0.2044 \mathrm{x}+2.1323$ & 0.122 \\
$\mathbf{Z n}$ & $\mathrm{y}=-0.79 \mathrm{x}+7.401$ & 0.444 \\
$\mathbf{C u}$ & $\mathrm{y}=-0.0097 \mathrm{x}+0.2649$ & 0.006 \\
$\mathbf{C d}$ & $\mathrm{y}=-0.0008 \mathrm{x}+0.0095$ & 0.0005 \\
$\mathbf{P b}$ & $\mathrm{y}=-0.0014 \mathrm{x}+0.0425$ & 0.001 \\
\hline
\end{tabular}

The Ni concentrations of the control $\mathrm{CaCO}_{3}$ neutralised AMD were higher than the original raw AMD concentration below $\mathrm{pH}$ 7.5. The high Ni concentration is inexplicable and may be due to experimental or analytical error. This result however does show that adsorption of $\mathrm{Ni}$ to calcite was minimal, even up to $\mathrm{pH}$ 8, as supported by Lakshtanov (2007). The adsorption of Ni to metal hydroxides does not appear to occur in calcite rich $\mathrm{CaCO}_{3}$ neutralised AMD.

The concentration of $\mathrm{Zn}$ was significantly higher after neutralisation by $\mathrm{CaCO}_{3}$, compared to $\mathrm{Ca}(\mathrm{OH})_{2}$ or $\mathrm{NaOH}$. Zinc does have a moderate adsorption affinity for calcite (GarcíaSánchez and Álvarez-Ayuso 2002), thou not as strong as its adsorption affinity for metal hydroxides. As the $\mathrm{Zn}$ concentration continues to decrease out to $\mathrm{pH} 8, \mathrm{Zn}$ removal may be occurring due to hydroxide precipitation (Kelly 1988).

Copper has a high affinity for adsorption to the calcite surface (Lee, Elzinga et al. 2005). Figure 7-6 shows a lower $\mathrm{Cu}$ concentration in $\mathrm{CaCO}_{3}$ neutralised AMD (compared to $\mathrm{NaOH}$ and $\mathrm{Ca}(\mathrm{OH})_{2}$ neutralised), probably due to a high degree of $\mathrm{Cu}$ sorption to 
abundant calcite. The $\mathrm{Cu}$ concentration was independent of $\mathrm{pH}$ over the range investigated, suggesting $\mathrm{Cu}$ adsorption to calcite occurred below pH 6.

A slightly lower $\mathrm{Cd}$ concentration was shown on Figure 7-6 for $\mathrm{CaCO}_{3}$ neutralised AMD compared to $\mathrm{Ca}(\mathrm{OH})_{2}$ neutralised AMD. Garcia-Sanchez (2002) determined a Cd-calcite sorption capacity of $5.5 \mathrm{mg} / \mathrm{g}$, which is relatively high considering the neutralised $\mathrm{Cd}$ concentration was less than $0.004 \mathrm{mg} / \mathrm{L}$. Thus, adsorption of $\mathrm{Cd}$ to calcite slightly improved Cd removal from neutralised AMD, compared to $\mathrm{Ca}(\mathrm{OH})_{2}$ conditions.

The concentration of $\mathrm{Pb}$ was slightly higher for $\mathrm{CaCO}_{3}$ neutralised AMD compared to $\mathrm{Ca}(\mathrm{OH})_{2}$. The $\mathrm{Pb}$ sorption capacity to calcite is relatively low (Rouff, Elzinga et al. 2005). The calcite dominance may therefore have offset the high $\mathrm{Pb}$ sorption affinity for metal oxide precipitates.

As discussed in section 5.2., between 1044 and $936 \mathrm{mg}$ of unreacted $\mathrm{CaCO}_{3}$ per litre remained after $\mathrm{AMD}$ neutralisation. The $\mathrm{CaCO}_{3}$ was densely packed, meaning that not all calcite surfaces would be available for metal adsorption. Thus the quantities of metal removed by calcite adsorption in this report may be low compared to the literature.

The sorption preferences of the abundant calcite appear to have dominated the total sorption characteristics of $\mathrm{CaCO}_{3}$ neutralised AMD conditions. Both $\mathrm{Pb}$ and $\mathrm{Zn}$ showed a very high affinity for adsorption to hydroxide precipitates in the $\mathrm{NaOH}$ and $\mathrm{Ca}(\mathrm{OH})_{2}$ neutralised control standard trials. Significantly less adsorption of both metals occurred in the calcite dominated sludge formed by $\mathrm{CaCO}_{3}$ neutralisation, resulting in greater residual dissolved metal concentrations. 


\subsubsection{2 $\mathrm{CaCO}_{3}$ Metal Removal}

Figure 7-7 shows the residual metal concentrations after HS addition and neutralisation. $\mathrm{NB}: \mathrm{CaCO}_{3}$ metal removal data are not included in appendices due to their volume.
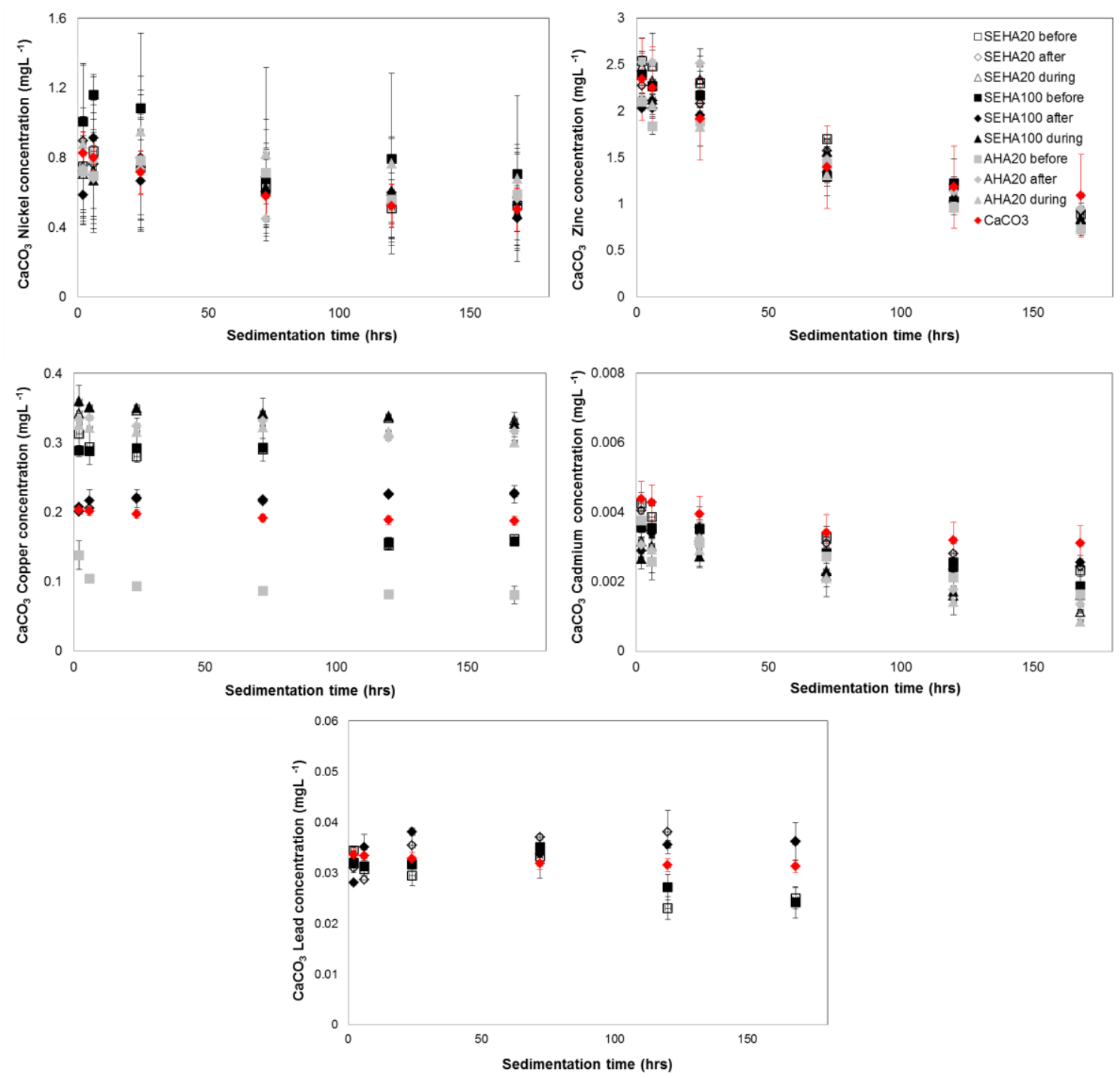

Figure 7-7: $\mathrm{CaCO}_{3}$ neutralised residual metal concentrations. Standard deviation error ( $\mathrm{n}=2$ ).

The results for $\mathrm{Ni}$ removal were inconclusive. The Ni concentrations after neutralisation were higher than the raw AMD concentration $(0.6 \mathrm{mg} / \mathrm{L})$ for almost all conditions. The W7 lignite (parent material of SEHA) had a low Ni content of $4 \mathrm{mg} / \mathrm{kg}$. A theoretical maximum Ni concentration in the SEHA would therefore be $0.8 \mathrm{mg} \mathrm{Ni}$ / L SEHA, using the extraction method discussed in section 3.3.1.1.2. A 1.5mL SEHA dose was used for the SEHA100 conditions, corresponding to a maximum Ni contribution from SEHA of $1.2 \mu \mathrm{g} / \mathrm{L}$. Thus, the high Ni concentration is inexplicable and may be due to experimental 
or analytical error. The standard deviation of the Ni data was on average $37 \%$ of the metal concentration. Therefore, most of these data were too variable to draw conclusions from.

The removal of $\mathrm{Zn}$ was not enhanced by HS addition. There does appear to be some HS enhancement at the 2, 6 hrs and 7 day sedimentation times. However, all HS dosed data still fall within the error bars of the control samples, suggesting the effects were statistically indeterminate.

Copper removal was not enhanced by HS addition. The AHA20 before-neutralisation condition did appear to enhance $\mathrm{Cu}$ removal by an average of $50 \%$, but that trend was not supported by either SEHA20 or SEHA100 before-neutralisation conditions. Therefore, the relatively high removal of $\mathrm{Cu}$ by adsorption to calcite (Lee, Elzinga et al. 2005) was not improved upon by HS dosing.

Removal of Cd appears to be enhanced by HS. All HS dosed conditions resulted in a lower $\mathrm{Cd}$ concentration than the control. All during-neutralisation conditions resulted in good Cd removal of $37-40 \%$. Increased SEHA dose concentration also resulted in greater Cd removal.

The metal removal data for $\mathrm{Pb}$ were incomplete. The data available show SEHA addition before neutralisation reduced $\mathrm{Pb}$ concentration by $7-10 \%$. However, as the $\mathrm{Pb}$ concentration is approaching the detection limit, these data may not be conclusive.

With the exception of $\mathrm{Cd}$, the addition of HS has no significant favourable effect on the removal of metals during $\mathrm{CaCO}_{3}$ neutralisation of AMD. Typically $\mathrm{HS}$ dosed metal concentrations were scattered around the control. This suggests that metal removal was dominated by adsorption to the approximately $1000 \mathrm{mg}$ of unreacted calcite conserved through the mixing and sedimentation process. The addition of even $100 \mathrm{mg}$ of SEHA was insufficient to compete with the calcite adsorption metal removal mechanism.

Table 7-6 shows the corresponding ANZECC level of protection for the treated metal concentrations for $1000 \mathrm{mg} / \mathrm{L} \mathrm{CaCO}_{3}$ hardness. 
HS - Metal Removal

Table 7-6: MSS level of protection of treated AMD

\begin{tabular}{ccc}
\hline & \multicolumn{2}{c}{$\mathrm{CaCO}_{3}$} \\
& Control & HS dosed \\
\hline $\mathbf{N i}$ & 95 & 95 \\
$\mathrm{Zn}$ & $<80$ & $<80$ \\
$\mathrm{Cu}$ & $<80$ & $<80$ \\
$\mathbf{C d}$ & 95 & 95 \\
$\mathbf{P b}$ & 95 & 95 \\
\hline
\end{tabular}

No HS dosed conditions improved the level of protection beyond the control condition. Both $\mathrm{Zn}$ and $\mathrm{Cu}$ concentrations after neutralisation were significantly above the recommended level for protection of $80 \%$ of species at $1000 \mathrm{mg} / \mathrm{L} \mathrm{CaCO}_{3}$. 


\subsection{Equilibrium Speciation}

Two sets of equilibrium speciation modelling results were generated; experimental and theoretical. The experimental speciation results were generated from equilibrium metal and HS concentrations measured from the HS jar testing samples. One speciation distribution was determined for the scenario with the greatest residual HS concentration for each alkalinity reagent; $\mathrm{NaOH}, \mathrm{Ca}(\mathrm{OH})_{2}$ and $\mathrm{CaCO}_{3}$. The theoretical speciation modelling was performed using only the control metal concentrations. A range of HS concentrations were used $(0.5$ to $100 \mathrm{mg} / \mathrm{L})$ to determine the degree of HS complexation that may occur when a neutralised AMD stream discharges into a naturally humic river.

Identifying metals bound to HS complexes was the main aim of speciation modelling. There were a total of six types of potential HS binding sites assumed in speciation modelling, as shown in Table 7-7, where Me is a complex forming cation.

\section{Table 7-7: HS binding sites}

\begin{tabular}{ll}
\hline HS binding site & Code \\
\hline Fulvic carboxylic & $\mathrm{HA1}-\mathrm{Me}(6)(\mathrm{aq})$ \\
Fulvic phenolic & $\mathrm{HA} 2-\mathrm{Me}(6)(\mathrm{aq})$ \\
Weakly (electrostatically) fulvic bound & $\mathrm{Me}+2 \mathrm{D}(6), \mathrm{Me}+1 \mathrm{D}(6)$ \\
Humic carboxylic & $\mathrm{HA} 1-\mathrm{Me}(7)(\mathrm{aq})$ \\
Humic phenolic & $\mathrm{HA} 2-\mathrm{Me}(7)(\mathrm{aq})$ \\
Weakly (electrostatically) humic bound & $\mathrm{Me}+2 \mathrm{D}(7), \mathrm{Me}+1 \mathrm{D}(7)$ \\
\hline
\end{tabular}

As noted in preceding metal removal sections, the $\mathrm{Cd}$ and $\mathrm{Pb}$ concentrations measured were often close to the detection level for the ICP-MS. These metals have been included in the experimental speciation modelling (despite the fact they may not actually be present) as they show the potential for HS complexation and were at sufficiently low concentrations to avoid interference with the analysis of other metals. The control neutralised concentrations of $\mathrm{Cd}$ and $\mathrm{Pb}$ were typically higher than the HS dosed samples. Therefore the $\mathrm{Cd}$ and $\mathrm{Pb}$ concentrations used in theoretical modelling are more reliable. 
HS - Metal Removal

\subsubsection{BTP NaOH Equilibrium Speciation Modelling}

\subsubsection{NaOH Experimental Speciation Modelling}

The highest residual HS concentration determined by $\mathrm{UV}$-vis for $\mathrm{NaOH}$ neutralised AMD was under the SEHA100 after-neutralisation scenario. The raw turbidity of this sample was 24.4 NTU due mostly to suspended/colloidal HS. Filtration resulted in a clear supernatant, suggesting the majority of HS were sufficiently large to be excluded by the $0.45 \mu \mathrm{m}$ filter. This observation was supported by the UV-vis analysis, which showed the HS concentration was $0.67 \mathrm{mg} / \mathrm{L}$.

Figure 7-8 shows the modelled speciation of the SEHA100 after metal concentrations, based on a HS concentration of $0.67 \mathrm{mg} / \mathrm{L}$.
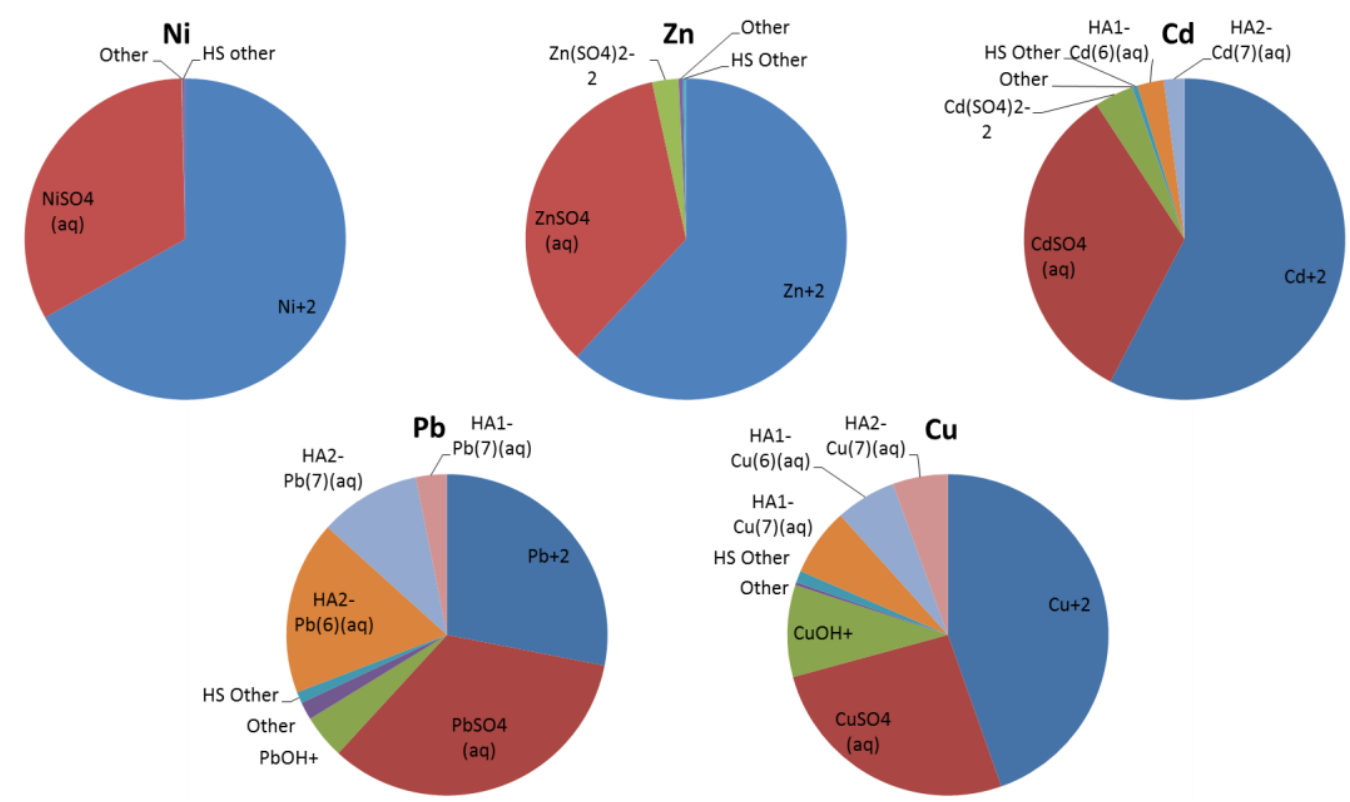

Figure 7-8: NaOH residual modelled metal speciation

Figure 7-8 shows very low complexation of $\mathrm{Ni}$ and $\mathrm{Zn}$, with both recording less than $1 \%$ HS speciation. The dominant modelled speciation of both $\mathrm{Ni}$ and $\mathrm{Zn}$ was dissolved.

Humic complexation of $\mathrm{Cd}$ was greater with over $5 \% \mathrm{Cd}$ in a HS complexed speciation. The distribution of HS was approximately equal between fulvic carboxylic and humic phenolic groups.

Lead formed HS complex the most readily among the metals investigated. Approximately $32 \%$ of $\mathrm{Pb}$ was $\mathrm{HS}$ complexed, with phenolic groups on both humic and fulvic acids favoured. A relatively high proportion of $\mathrm{Cu}$ was also HS complexed. Approximately 
$20 \%$ of $\mathrm{Cu}$ was complexed, primarily to carboxylic groups on both humic and fulvic acids. Both $\mathrm{Cu}$ and $\mathrm{Pb}$ have a high affinity for HS complexation (Milne, Kinniburgh et al. 2003), which results in a relatively high proportion of $\mathrm{Cu}$ and $\mathrm{Pb}$ complexed to $\mathrm{HS}$, even at low HS concentrations $(0.67 \mathrm{mg} / \mathrm{L})$.

\subsubsection{NaOH Theoretical Speciation Modelling}

The $\mathrm{NaOH}$ control metal concentrations were used for the theoretical HS speciation modelling at approximately $\mathrm{pH}$ 6.6. Figure 7-9 shows the theoretical speciation of HS complexed AMD neutralised by $\mathrm{NaOH}$ at a range of HS concentrations. NB: For simplicity total HS complexation is reported for theoretical speciation modelling, not the humic/fulvic and carboxylic/phenolic fractions.
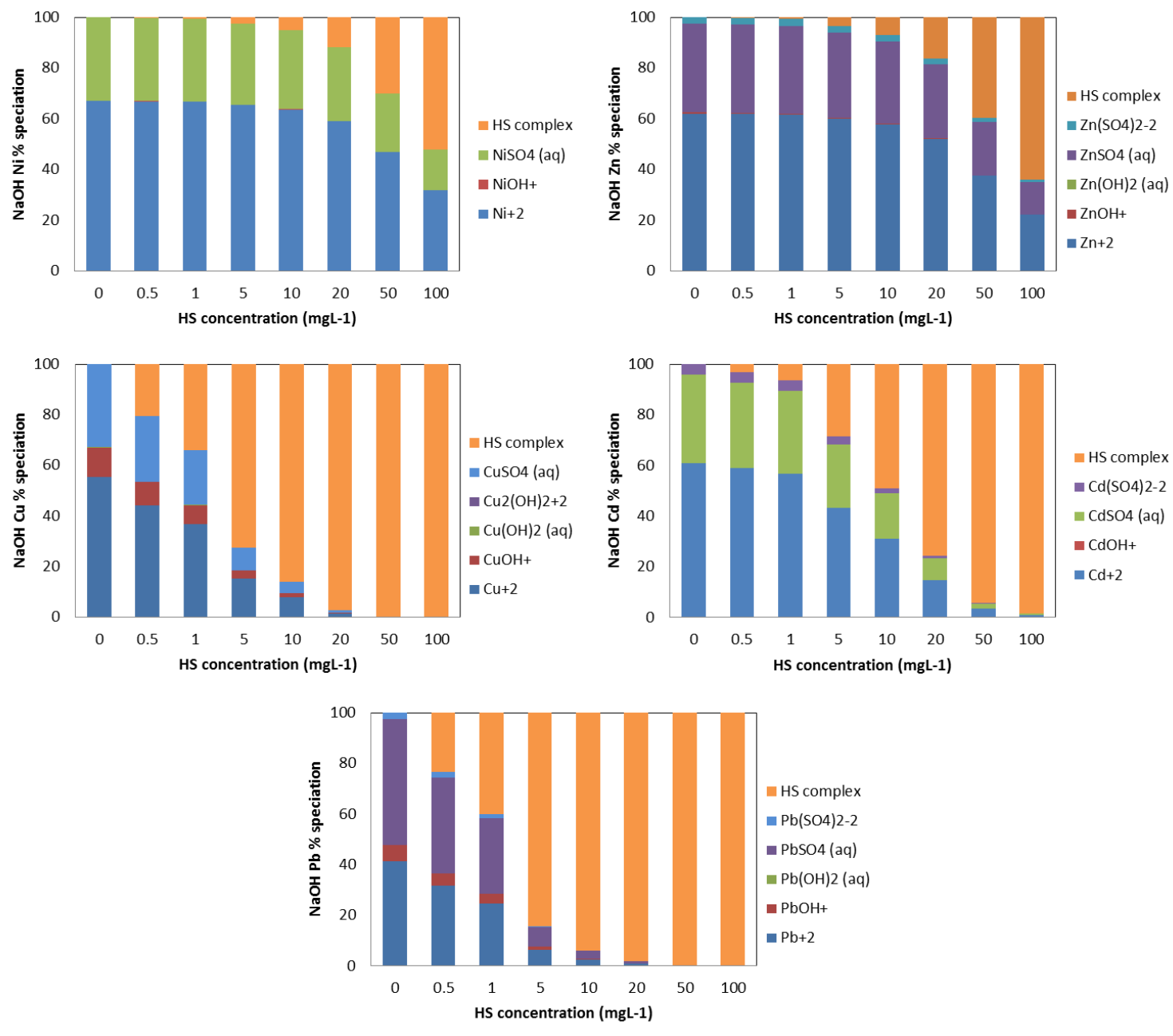

Figure 7-9: Theoretical modelled speciation of $\mathrm{NaOH}$ neutralised AMD

Figure 7-9 shows that both $\mathrm{Ni}$ and $\mathrm{Zn}$ have a relatively low affinity for HS complexation. At $20 \mathrm{mg} / \mathrm{L} \mathrm{HS}, 12$ and $16 \%$ of $\mathrm{Ni}$ and $\mathrm{Zn}$ were $\mathrm{HS}$ complexed, respectively. This 
increases to 52 and $64 \%$ at a concentration of $100 \mathrm{mg} / \mathrm{L} \mathrm{HS}$. Cadmium has a higher affinity for HS complexation, with 7 and 98\% complexation at 1 and $100 \mathrm{mg} / \mathrm{L} \mathrm{HS}$, respectively.

Copper and Lead formed HS complex readily, with 34 and $40 \%$ of $\mathrm{Cu}$ and $\mathrm{Pb}$ complexed at $1 \mathrm{mg} / \mathrm{L} \mathrm{HS}$. At a HS concentration of $50 \mathrm{mg} / \mathrm{L}$, over $99 \%$ of $\mathrm{Cu}$ and $\mathrm{Pb}$ were $\mathrm{HS}$ complexed. Due to high binding affinities and low concentrations, a high proportion of $\mathrm{Cu}$ and $\mathrm{Pb}$ may be complexed even at relatively low HS concentration, as shown in section 7.3.1.1.

The dominant cation in $\mathrm{NaOH}$ neutralised scenarios was $\mathrm{Na}$, at a concentration of 375 $\mathrm{mg} / \mathrm{L}$. Sodium is a weakly electrostatically bound background electrolyte and may not compete for the fulvic and humic binding sites.
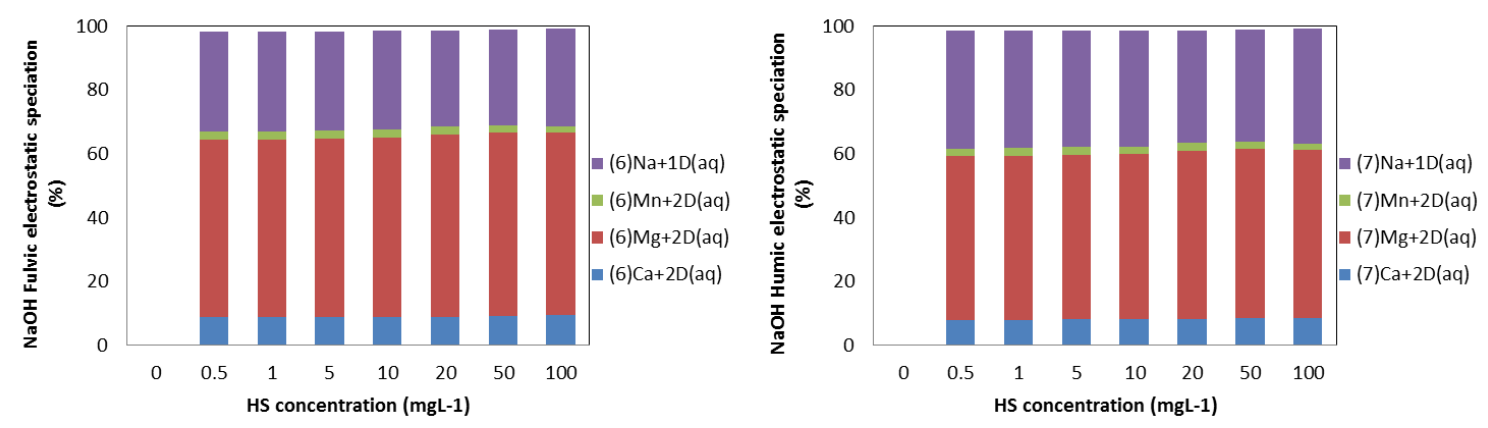

Figure 7-10: Na binding to fulvic and humic electrostatic sites

Figure 7-10 shows that 30 and $36 \%$ of the fulvic and humic electrostatic sites were occupied by $\mathrm{Na}$, respectively. None of the target metals of this study significantly partition into the electrostatic fraction, thus $\mathrm{Na}$ competition for these HS binding sites would be negligible.
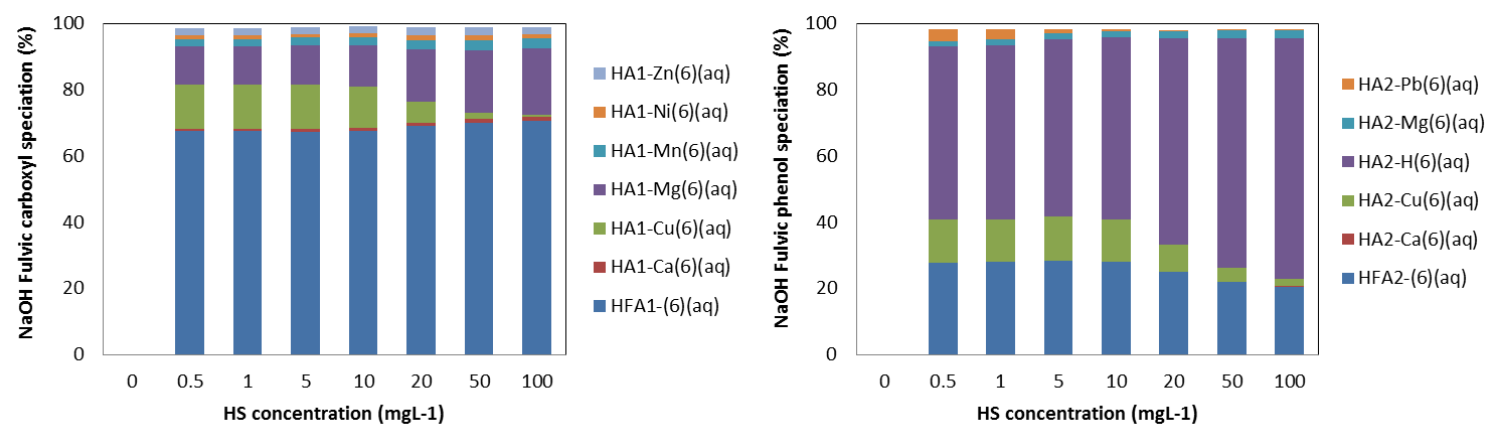

Figure 7-11: NaOH Fulvic carboxylic and phenolic complexation 
Figure 7-11 shows the metal complexation to fulvic carboxylic and fulvic phenolic groups. Complexation to fulvic carboxylic groups ranged from 13, 1.5 and $2.5 \%$ for $\mathrm{Cu}$, $\mathrm{Ni}$ and $\mathrm{Zn}$, respectively. The fulvic phenolic groups were occupied by $\mathrm{Cu}$ and $\mathrm{Pb}$ at 15 and $3.6 \%$, respectively.
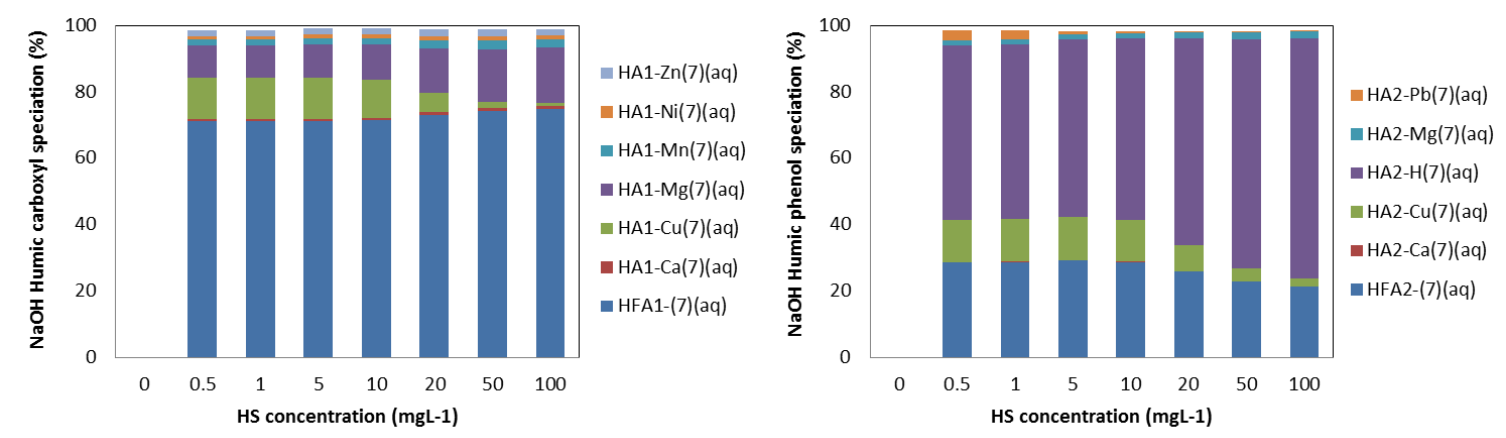

Figure 7-12: NaOH Humic carboxylic and phenolic complexation

Figure 7-12 shows the metal complexation of humic carboxylic and humic phenolic groups. A similar degree of humic carboxylic complexation to fulvic carboxylic groups was shown with $12,1.4$ and $2.2 \% \mathrm{Cu}, \mathrm{Ni}$ and $\mathrm{Zn}$ complexation. The humic phenolic groups were complexed by $13 \% \mathrm{Cu}$ and $3 \% \mathrm{~Pb}$. The maximum proportion of fulvic or humic groups occupied by $\mathrm{Ca}$ for the $\mathrm{NaOH}$ neutralised conditions was below $1.2 \%$.

The partition of the target metals into the fulvic and humic groups during $\mathrm{NaOH}$ neutralisation was considered the control theoretical speciation, as $\mathrm{Na}$ does not compete for these binding sites. The speciation of metals obtained from $\mathrm{Ca}(\mathrm{OH})_{2}$ and $\mathrm{CaCO}_{3}$ neutralised samples are therefore compared to the $\mathrm{NaOH}$ control. 
HS - Metal Removal

\subsubsection{BTP Ca(OH)2 Equilibrium Speciation Modelling}

\subsubsection{1 $\mathrm{Ca}(\mathrm{OH})_{2}$ Experimental Speciation Modelling}

The highest residual HS concentration of the $\mathrm{Ca}(\mathrm{OH})_{2}$ trials was again the SEHA 100 after-neutralisation condition at $0.84 \mathrm{mg} / \mathrm{L}$. Figure 7-13 shows the experimental speciation of the $\mathrm{Ca}(\mathrm{OH})_{2}$ conditions.

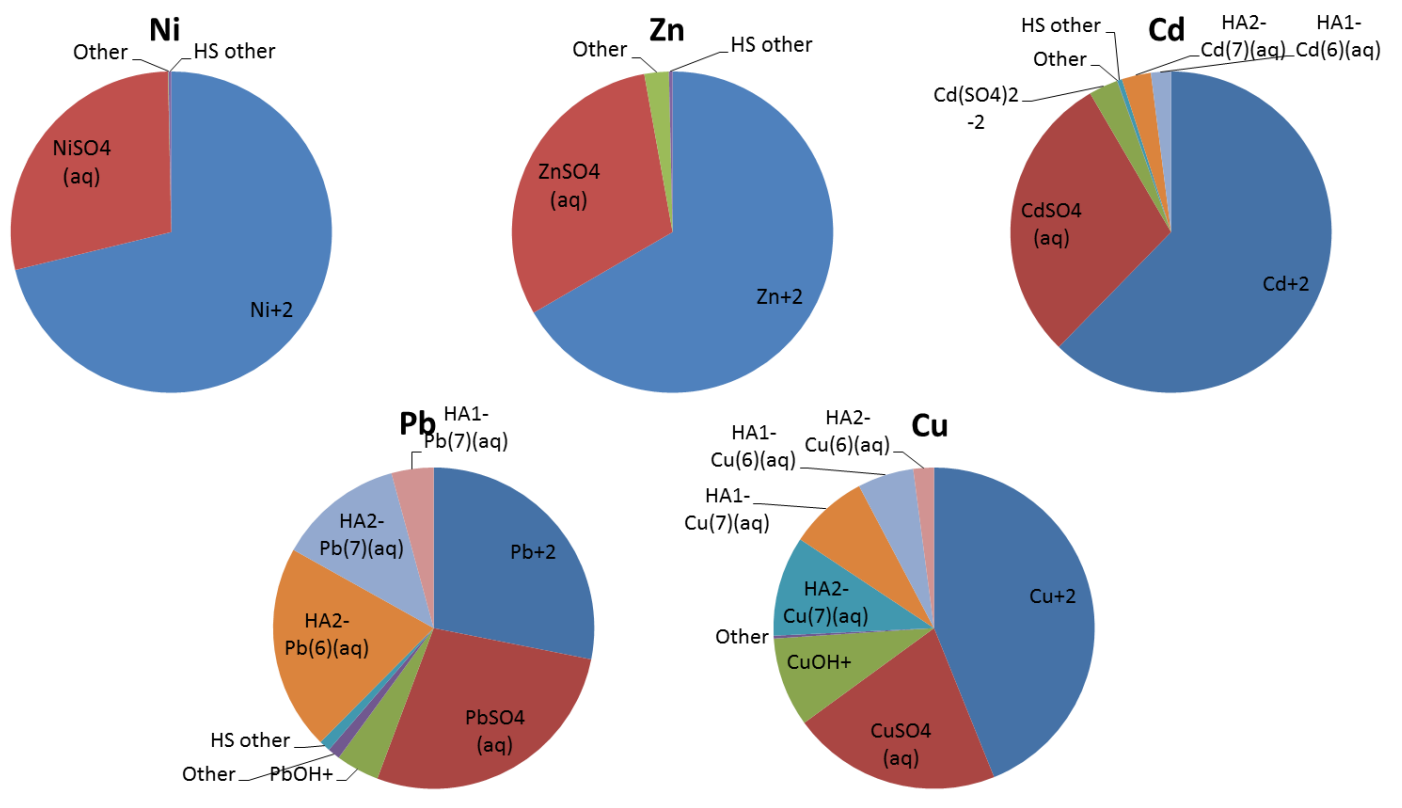

Figure 7-13: $\mathrm{Ca}(\mathrm{OH})_{2}$ residual modelled metal speciation

Again, both $\mathrm{Ni}$ and $\mathrm{Zn}$ showed a low affinity for $\mathrm{HS}$ with less than $1 \%$ forming $\mathrm{HS}$ complexes. Complexation of $\mathrm{Cd}$ was slightly higher, as approximately $5 \%$ of $\mathrm{Cd}$ formed HS complexes.

Conversely, a high percentage of $\mathrm{Cu}$ and $\mathrm{Pb}$ was $\mathrm{HS}$ complexed. Over 38 and $25 \%$ of $\mathrm{Pb}$ and $\mathrm{Cu}$ were in $\mathrm{HS}$ speciation. This was higher than the $\mathrm{NaOH} \mathrm{Pb}$ and $\mathrm{Cu} H S$ complexation, probably because the $\mathrm{Ca}(\mathrm{OH})_{2} \mathrm{HS}$ concentration was higher at $0.84 \mathrm{mg} / \mathrm{L}$ compared to $0.67 \mathrm{mg} / \mathrm{L}$ for $\mathrm{NaOH}$. Lead showed a high complexation affinity for phenolic groups while $\mathrm{Cu}$ complexed to both carboxylic and phenolic groups. 


\subsubsection{2 $\mathrm{Ca}(\mathrm{OH})_{2}$ Theoretical Speciation Modelling}

Figure 7-14 shows the theoretical speciation of HS complexed AMD neutralised by $\mathrm{Ca}(\mathrm{OH})_{2}$ at a range of $\mathrm{HS}$ concentrations $(0.5-100 \mathrm{mg} / \mathrm{L})$.
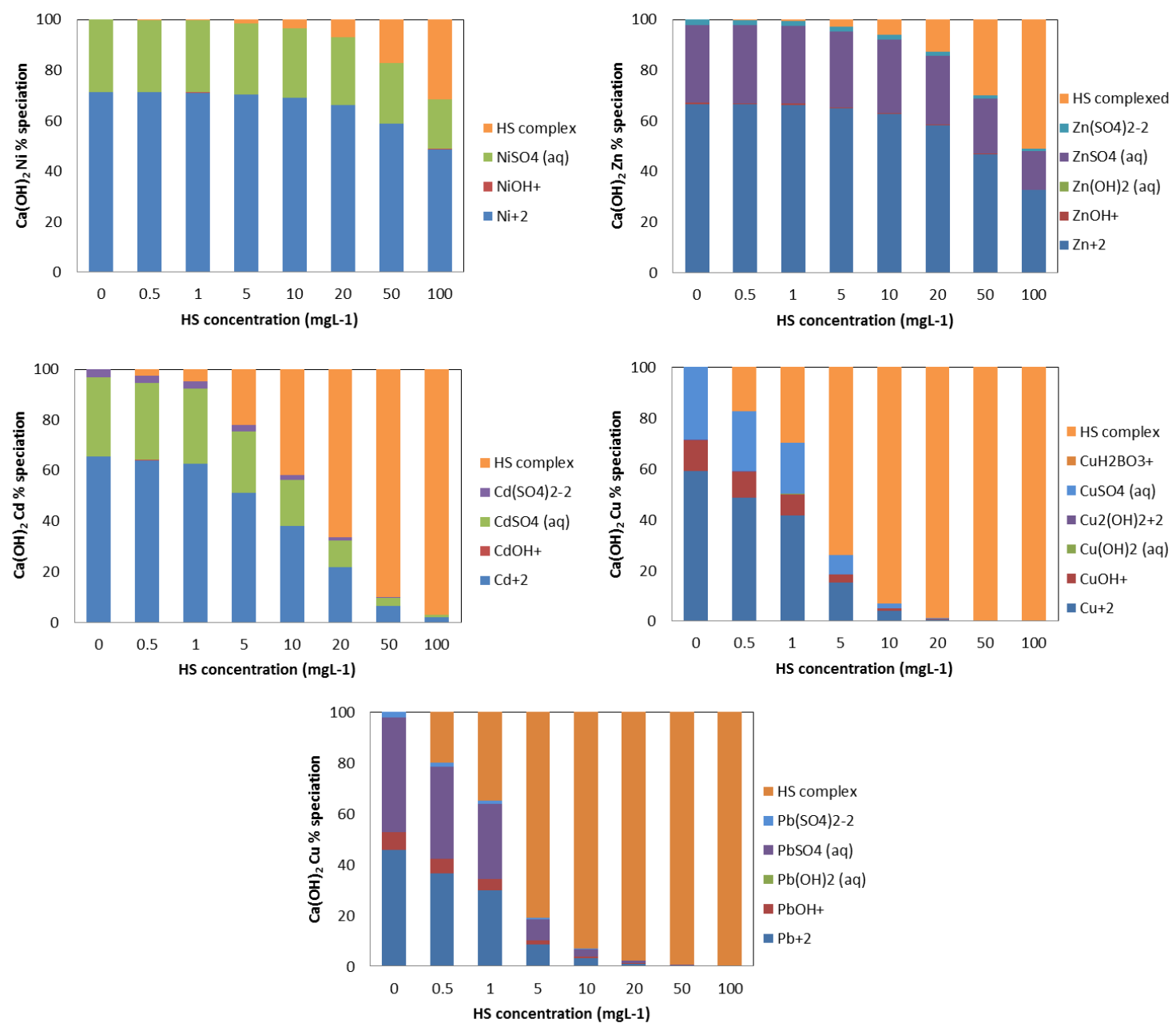

Figure 7-14: Theoretical modelled speciation of $\mathrm{Ca}(\mathrm{OH})_{2}$ neutralised AMD

Figure 7-14 shows similar trends for the speciation of $\mathrm{Ca}(\mathrm{OH})_{2}$ neutralised metals as in the $\mathrm{NaOH}$ case. At $20 \mathrm{mg} / \mathrm{L} \mathrm{HS}, 7$ and $13 \%$ of $\mathrm{Ni}$ and $\mathrm{Zn}$ were in $\mathrm{HS}$ complexed speciation, respectively. This increased to 31 and $51 \%$ at a concentration of $100 \mathrm{mg} / \mathrm{L}$ HS.

Cadmium showed 5 and 97\% complexation at 1 and $100 \mathrm{mg} / \mathrm{L}$ HS respectively. Copper and Lead formed HS complex readily, with 30 and $35 \%$ complexed at $1 \mathrm{mg} / \mathrm{L} \mathrm{HS}$, respectively. At a $\mathrm{HS}$ concentration of $50 \mathrm{mg} / \mathrm{L}$, over $99 \%$ of $\mathrm{Cu}$ and $\mathrm{Pb}$ were $\mathrm{HS}$ complexed. 
Competition for binding sites on HS from background Ca was investigated as the concentration of $\mathrm{Ca}$ was $386 \mathrm{mg} / \mathrm{L}$, due to $\mathrm{Ca}(\mathrm{OH})_{2}$ dissolution during neutralisation. Calcium formed complexes with all six HS functional groups, resulting in binding site competition with target metals to varying degrees.
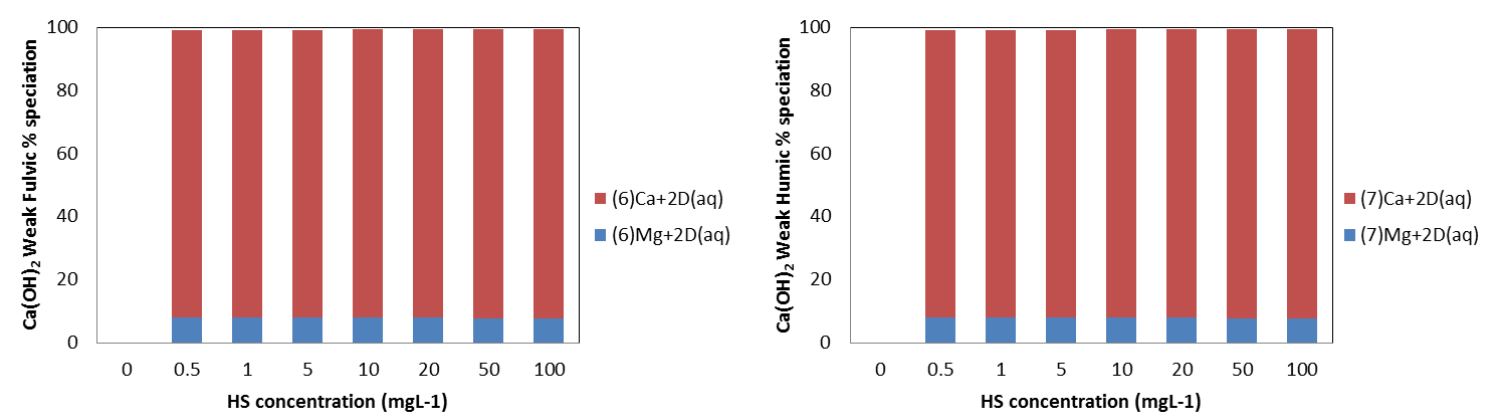

Figure 7-15: $\mathrm{Ca}(\mathrm{OH})_{2}$ derived Ca binding to fulvic and humic electrostatic sites

Figure 7-15 shows that the high $\mathrm{Ca}$ concentration caused significant competition for electrostatic binding sites. The electrostatic binding sites were over 90\% complexed with Ca. Nevertheless, these sites were shown to have a lower affinity for the target metals in section 7.3.1.2. Thus, Ca competition to electrostatic binding sites would not affect total metal-HS complexation significantly.
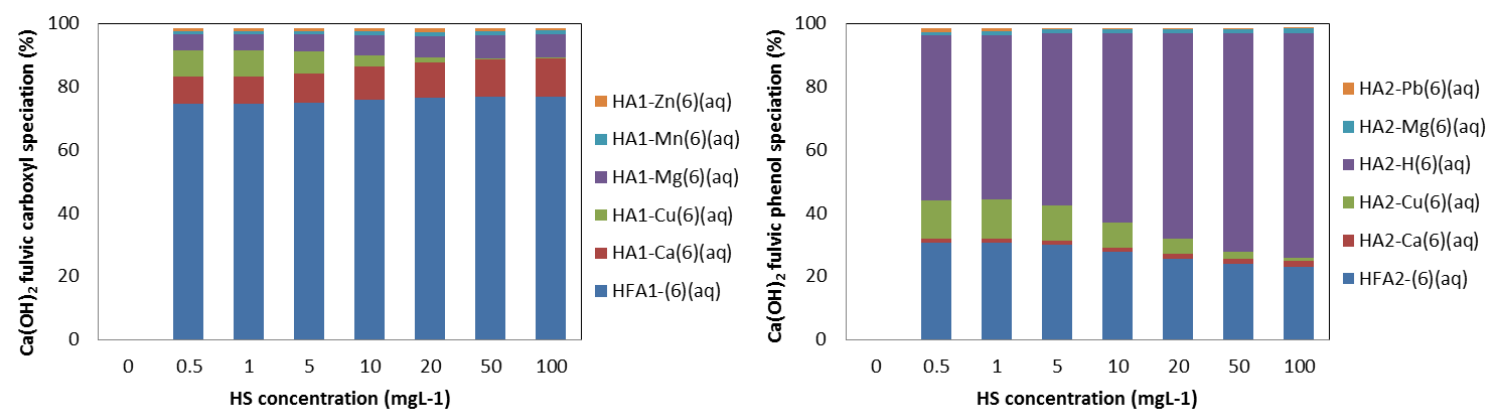

Figure 7-16: $\mathrm{Ca}(\mathrm{OH})_{2}$ fulvic carboxylic and phenolic complexation

Figure 7-16 shows a reduction in the amount of complexed target metals at fulvic carboxylic sites. The maximum percent of fulvic carboxylic bound $\mathrm{Cu}, \mathrm{Zn}$ and $\mathrm{Ni}$ decreased from the control to 8.3, 1.1 and below $1 \%$, respectively. The complexation of $\mathrm{Ca}$ to carboxylic sites increased to $12 \%$, suggesting $\mathrm{Ca}$ competition had reduced complexation of the target metals. The fulvic phenol group was less affected as $\mathrm{Ca}$ binding only totalled $1.8 \%$ of the sites. Therefore $\mathrm{Cu}$ and $\mathrm{Pb}$ complexation were still relatively high at 12 and $1.3 \%$, respectively. 

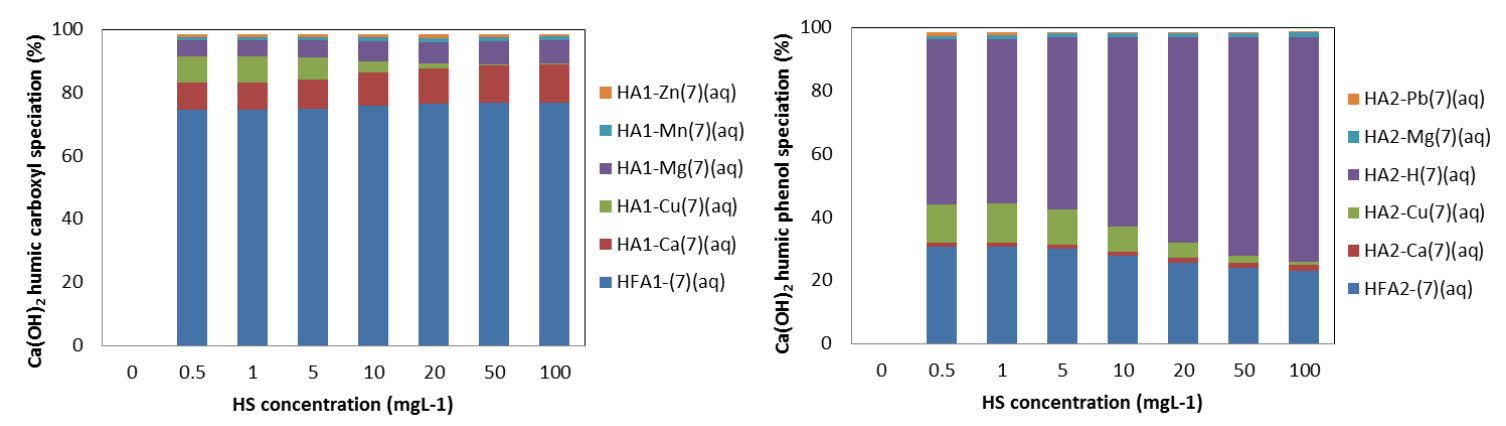

Figure 7-17: $\mathrm{Ca}(\mathrm{OH})_{2}$ humic carboxylic and phenolic complexation

Figure 7-17 shows that Ca competed for binding at the humic carboxylic sites also, with complexation of $\mathrm{Cu}$ and $\mathrm{Zn}$ decreasing to 8.3 and $1.1 \%$, respectively due to $\mathrm{Ca}$ complexation of $12 \%$. Copper and $\mathrm{Pb}$ complexation at humic phenolic sites was relatively unaffected at 12 and $1.3 \%$, respectively; as Ca complexation was limited to $1.8 \%$ of groups.

The BTP speciation modelling showed that metal complexation was more controlled by the functional group (carboxylic or phenolic) of a reactive site than humic substance fraction (humic or fulvic). When complexation competition from background ions was low $(\mathrm{Na})$, carboxylic and phenolic groups complexed relatively equal amounts of metals, particularly $\mathrm{Cu}$. Binding competition from divalent background ions ( $\mathrm{Ca}$ ) resulted in minor decreased complexation at carboxylic sites, while phenolic sites were largely unaffected.

The SEHA product was shown to have a relatively high concentration of phenolic groups in section 4.1, compared to AHA. Thus, the speciation modelling and characterisation of HS suggests that the additional removal of $\mathrm{Cu}$ by $\mathrm{HS}$ dosing observed for some BTP conditions was possibly due to complexation of $\mathrm{Cu}$ at phenolic functional groups. 
HS - Metal Removal

\subsubsection{MSS $\mathrm{CaCO}_{3}$ Equilibrium Speciation Modelling}

\subsubsection{1 $\mathrm{CaCO}_{3}$ Experimental Speciation Modelling}

The highest HS concentration for the $\mathrm{CaCO}_{3}$ neutralised samples was $1.16 \mathrm{mg} / \mathrm{L}$ and occurred under the $20 \mathrm{mg} / \mathrm{L}$ SEHA before-neutralisation scenario. The average metal concentrations of the SEHA20 before-neutralisation, $\mathrm{CaCO}_{3}$ neutralised condition was used for speciation modelling. The average metal concentrations were approximately the same as the metal concentrations after 72 hours sedimentation.

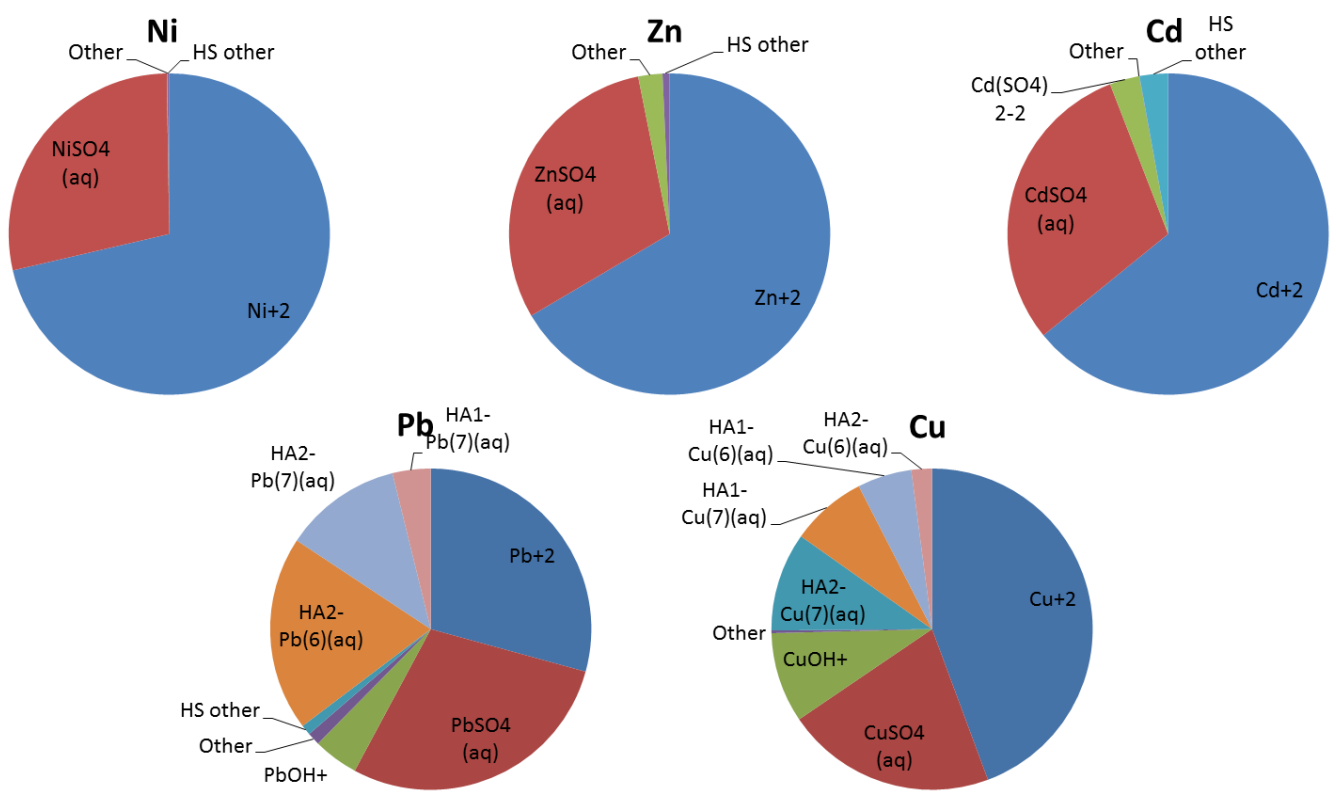

Figure 7-18: $\mathrm{CaCO}_{3}$ residual metal speciation

Figure 7-18 shows both $\mathrm{Ni}$ and $\mathrm{Zn}$ were complexed poorly with less than $1 \%$ of total metal in HS complexed speciation. HS complexation of Cd was higher at approximately $3 \%$. Complexation of $\mathrm{Pb}$ and $\mathrm{Cu}$ was higher with 36 and $25 \%$ in $\mathrm{HS}$ speciation, respectively. The percent of metals HS complexed was lower than the $\mathrm{NaOH}$ and $\mathrm{Ca}(\mathrm{OH})_{2}$ distributions, despite a higher HS concentration of $1.16 \mathrm{mg} / \mathrm{L}$. This was probably because the $\mathrm{CaCO}_{3}$ neutralised conditions had higher metal concentrations, so the ratio of HS to metal had decreased.

\subsubsection{2 $\mathrm{CaCO}_{3}$ Theoretical Speciation Modelling}

The theoretical speciation of $\mathrm{CaCO}_{3}$ was determined using the average $\mathrm{CaCO}_{3}$ metal concentrations over all the sedimentation samples. Figure 7-19 shows the theoretical speciation of $\mathrm{HS}$ dosed $\mathrm{CaCO}_{3} \mathrm{AMD}$. 

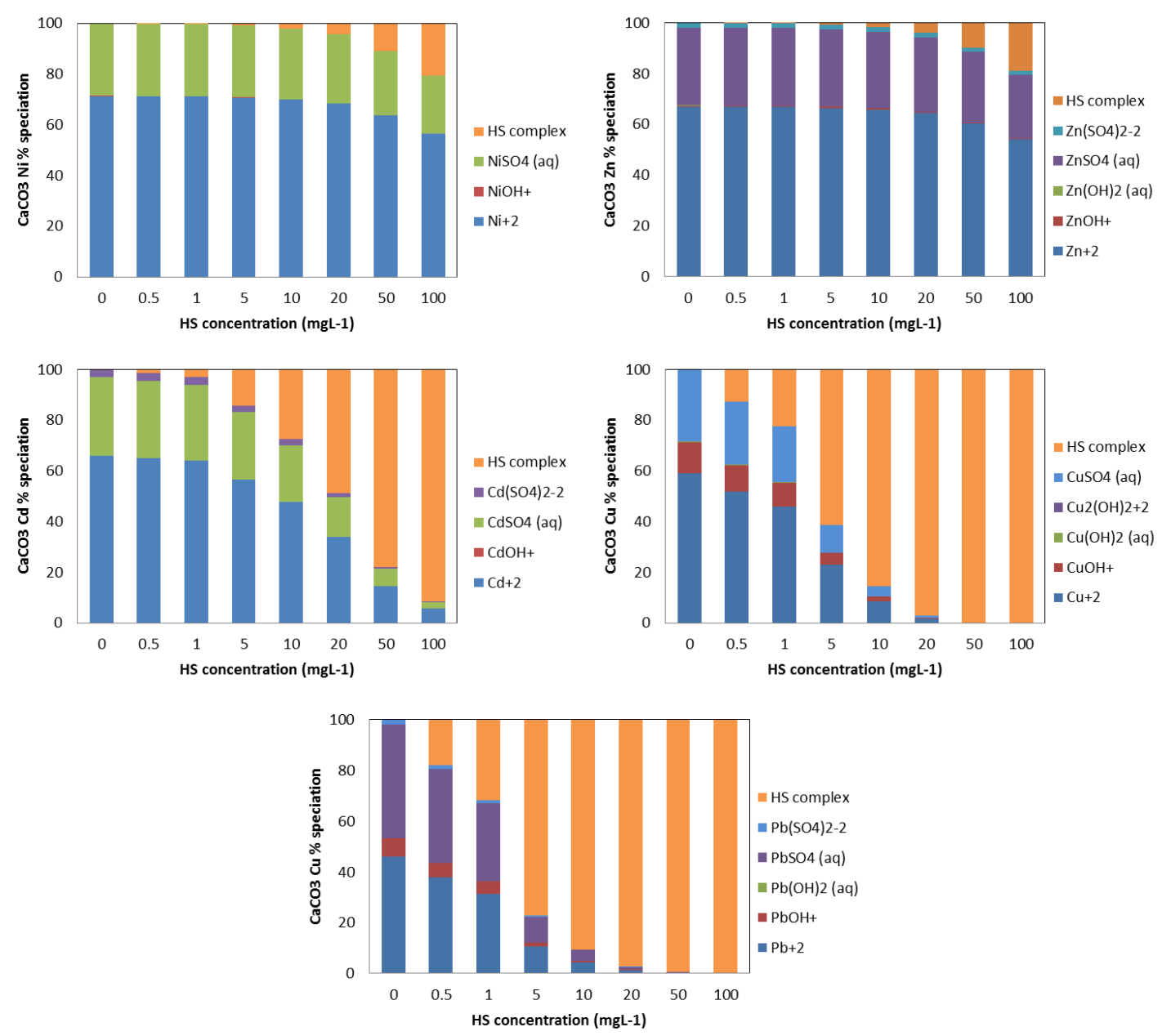

Figure 7-19: Theoretical modelled speciation of $\mathrm{CaCO}_{3}$ neutralised $\mathrm{AMD}$

At $20 \mathrm{mg} / \mathrm{L} \mathrm{HS}, 4 \%$ of both $\mathrm{Ni}$ and $\mathrm{Zn}$ were in $\mathrm{HS}$ complexed speciation, respectively. This increased to $20 \%$ at a concentration of $100 \mathrm{mg} / \mathrm{L} \mathrm{HS}$.

Cadmium showed 3 and 91\% complexation at 1 and $100 \mathrm{mg} / \mathrm{L} \mathrm{HS}$, respectively. Copper and Lead again formed $\mathrm{HS}$ complex readily, with 22 and $32 \%$ of $\mathrm{Cu}$ and $\mathrm{Pb}$ complexed at $1 \mathrm{mg} / \mathrm{L} \mathrm{HS}$. At a HS concentration of $50 \mathrm{mg} / \mathrm{L}$, over $99 \%$ of $\mathrm{Cu}$ and $\mathrm{Pb}$ were $\mathrm{HS}$ complexed.

Figure 7-19 suggests that there is less HS complexation of the target metal group for a given HS concentration when neutralised by $\mathrm{CaCO}_{3}$. However, the metal concentrations of $\mathrm{CaCO}_{3}$ neutralised AMD were typically higher than their $\mathrm{NaOH}$ and $\mathrm{Ca}(\mathrm{OH})_{2}$ neutralised counterparts. Therefore, a lower proportion of the total metal concentration is bound to the fixed number of metal complexation sites. Competition for HS binding sites from $\mathrm{Ca}$ produced by dissolution of $\mathrm{CaCO}_{3}$ reduced the HS complexation of some target metals as discussed in section 7.3.2.2. 

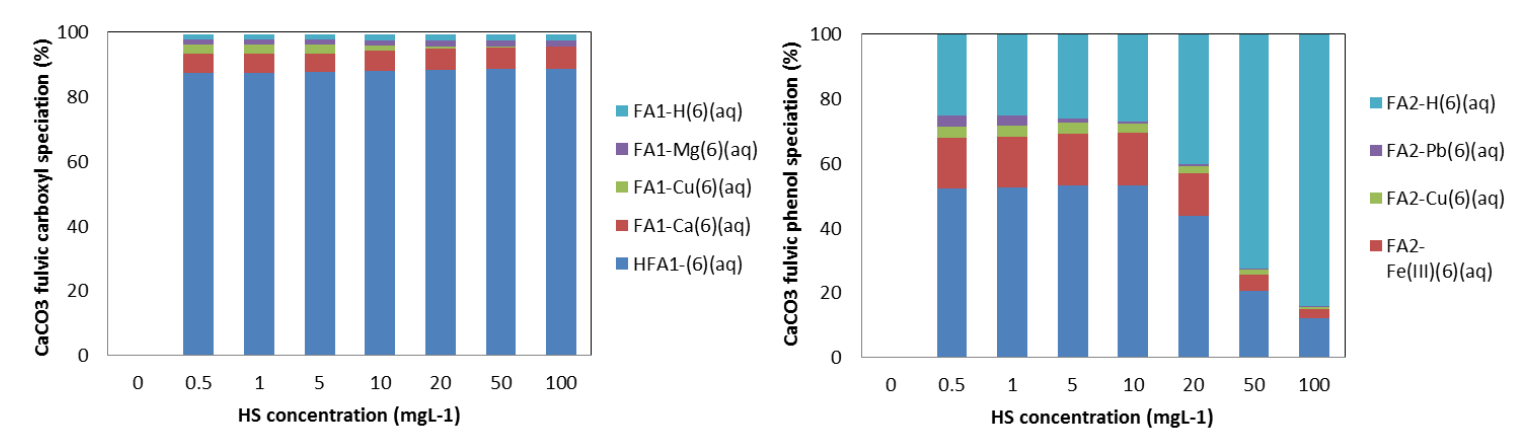

Figure 7-20: $\mathrm{CaCO}_{3}$ fulvic carboxylic and phenolic complexation

Figure 7-20 shows Ca competition for fulvic carboxylic groups. The maximum percent of fulvic carboxylic bound $\mathrm{Cu}$ was $2.9 \%$ while $\mathrm{Zn}$ and $\mathrm{Ni}$ were both below $1 \%$. The complexation of $\mathrm{Ca}$ was $7 \%$, suggesting $\mathrm{Ca}$ competition had reduced complexation of the target metals. The fulvic phenol group was less affected as Ca complexation occupied less than $1 \%$ of sites. Over $3 \%$ of both $\mathrm{Cu}$ and $\mathrm{Pb}$ were complexed to fulvic phenol groups.
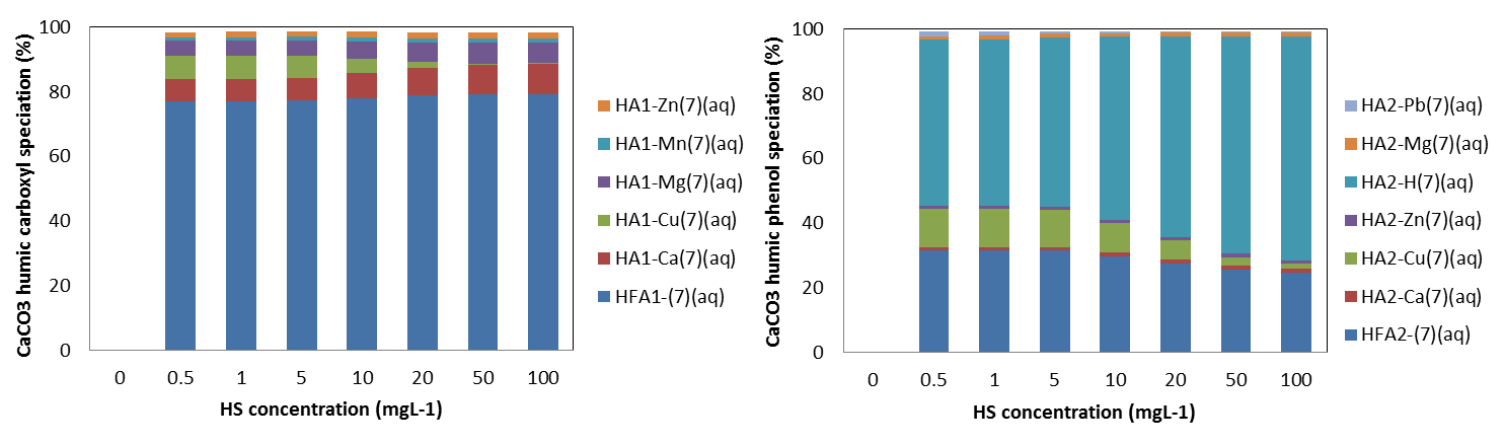

Figure 7-21: $\mathrm{CaCO}_{3}$ humic carboxylic and phenolic complexation

Figure 7-21 shows that Ca competed for binding at the humic carboxylic sites, occupying $9 \%$ of sites. Binding to $\mathrm{Cu}$ and $\mathrm{Zn}$ was $7 \%$ and $2 \%$, respectively. Binding competition for the humic phenol sites was relatively low as Ca competition was only $1.4 \%$. Thus, $\mathrm{Cu}$ and $\mathrm{Pb}$ complexation was 12 and $1.4 \%$ of humic phenolic sites.

The HS complexation of metals during $\mathrm{CaCO}_{3}$ neutralisation of AMD shows the same dependence on carboxylic and phenolic functional groups as $\mathrm{Ca}(\mathrm{OH})_{2}$ neutralised AMD, with Ca competition reducing the complexation capacity of carboxylic sites. Equilibrium modelling identified phenolic groups as dominating the complexation of $\mathrm{Cd}$ (data not shown). The high phenolic concentration of SEHA may therefore have caused the enhanced removal of $\mathrm{Cd}$ observed for $\mathrm{CaCO}_{3}$ neutralised AMD. 


\subsection{Summary - review}

The metal concentration of HS dosed AMD supernatants varied depending on the dose order and the alkalinity reagent used. HS dosed metal concentrations were compared to control metal concentrations at the same $\mathrm{pH}$ to identify any $\mathrm{HS}$ enhancement. Neutralisation of AMD by all alkalinity reagent only conditions (control) resulted in a decrease in all metals from raw AMD concentration (except $\mathrm{Ni}$ neutralised by $\mathrm{CaCO}_{3}$ ), due to a range of mechanisms including precipitation, co-precipitation and adsorption.

Most $\mathrm{HS}$ dosed conditions neutralised by $\mathrm{NaOH}$ resulted in $\mathrm{Ni}, \mathrm{Zn}$, and $\mathrm{Pb}$ concentrations that exceeded the control, suggesting that HS complexation of metals actually interfered with the control metal removal mechanisms. The SEHA20 and SEHA100 duringneutralisation conditions enhanced the removal of $\mathrm{Cu}$ by over $50 \%$. The high removal of $\mathrm{Cu}$ was attributed to a high affinity of $\mathrm{Cu}$ for HS (Čežíková, Kozler et al. 2001; Milne, Kinniburgh et al. 2003; Pehlivan and Arslan 2007), particularly the phenolic reactive sites (as shown by equilibrium modelling) which are well represented on SEHA. Data for Cd were indeterminate, due to overlapping error bars between control and HS dosed samples. Residual turbidity due to suspended/colloidal HS could potentially result in facilitated transport of metals. The SEHA100 after-neutralisation by $\mathrm{NaOH}$ condition was significantly discoloured and turbid. However, after filtration the sample was colourless and metal analysis did not show an elevated metal concentrations. Therefore, HS complexed metals in the dissolved phase would not facilitate contaminant transport.

Neutralisation of $\mathrm{AMD}$ by $\mathrm{Ca}(\mathrm{OH})_{2}$ resulted in slightly higher control metal concentrations than $\mathrm{NaOH}$, possibly due to $\mathrm{Ca}$ competition for sorption sites on precipitated Fe/Al hydroxides.. The effects of HS dosing on metal removal were therefore more pronounced, though $\mathrm{Ni}$ and $\mathrm{Pb}$ concentrations were not decreased by HS dosing. The SEHA20 during- and after-neutralisation conditions enhanced the removal of $\mathrm{Cu}$ by 55 and $66 \%$, respectively. The Cd removal was also enhanced for all HS dose conditions, with $80 \%$ removal by SEHA20 after-neutralisation. Cadmium has a relatively high complexation affinity (Čežíková, Kozler et al. 2001; Milne, Kinniburgh et al. 2003) proportional to its concentration in AMD. Equilibrium modelling showed that $\mathrm{Cd}$ was less selective, binding to both carboxylic and phenolic reactive groups. The $\mathrm{Ca}(\mathrm{OH})_{2}$ results suggested the removal of Zn was enhanced by HS dosing, however, these results 
are questionable as both the literature review and speciation modelling showed that $\mathrm{Zn}$ had a low affinity for HS complexation.

Neutralisation of $\mathrm{AMD}$ by $\mathrm{CaCO}_{3}$ resulted in higher concentrations of $\mathrm{Ni}, \mathrm{Zn}$ and $\mathrm{Pb}$; but lower concentrations of $\mathrm{Cu}$ and $\mathrm{Cd}$, compared to $\mathrm{Ca}(\mathrm{OH})_{2}$ neutralisation. Due to the low dissolution of $\mathrm{CaCO}_{3}$, the dominant particulate species was calcite $\left(\mathrm{CaCO}_{3}\right)$. Thus, the adsorption affinities of the target metals for calcite dominated the removal of metals by adsorption. Both $\mathrm{Cu}$ and $\mathrm{Cd}$ had high calcite binding affinities and thus were present at a lower concentration in control samples (García-Sánchez and Álvarez-Ayuso 2002). Subsequent HS dosing resulted in up to $40 \%$ additional removal of $\mathrm{Cd}$ for the duringneutralisation condition, while the removal of other metals was not enhanced by HS dosing.

The addition of $\mathrm{HS}$ was expected to enhance $\mathrm{Pb}$ removal as $\mathrm{Pb}$ has a high complexation affinity for both HS and precipitated metal hydroxides (Čežíková, Kozler et al. 2001; Lee, Bigham et al. 2002; Milne, Kinniburgh et al. 2003; Pehlivan and Arslan 2007). The concentrations of $\mathrm{Pb}$ in the raw AMD were only four times the detection limit, which was relatively high at $0.024 \mathrm{mg} / \mathrm{L}$. Thus, any interaction between $\mathrm{Pb}$ and $\mathrm{HS}$ or metal hydroxides was difficult to identify. The addition of $\mathrm{HS}$ showed no Ni removal enhancement which is supported by the literature (Čežíková, Kozler et al. 2001; Pehlivan and Arslan 2007) and speciation modelling which state $\mathrm{Ni}$ had a low complexation affinity for HS. 



\section{Chapter 8. Feasibility/Markets}

The feasibility/markets chapter is divided into two sections

Section 1: Feasibility. This section discusses the cost: benefit analysis of Solid Energy Humic Acid (SEHA) as a polymer for the removal of residual $\mathrm{CaCO}_{3}$ suspended solids (SS). The cost: benefit of HS as an enhancing agent for metal removal is also discussed.

Section 2: Market review. This section briefly identifies other markets (primarily agricultural) which currently use humic substances (HS) as a product to enhance economic performance. 
Feasibility/Markets

\subsection{Feasibility}

This section compares the operational cost: benefit of HS dosing during acid mine drainage (AMD) neutralisation. The cost: benefit of HS as a polymer for removal of $\mathrm{CaCO}_{3} \mathrm{SS}$ is discussed first. The cost: benefit of HS enhanced metal removal is then discussed.

\subsubsection{Cost: benefit of HS as a Polymer}

Figure 8-1 shows the SEHA and polymer doses used for the cost: benefit analysis of SEHA application as a polymer.

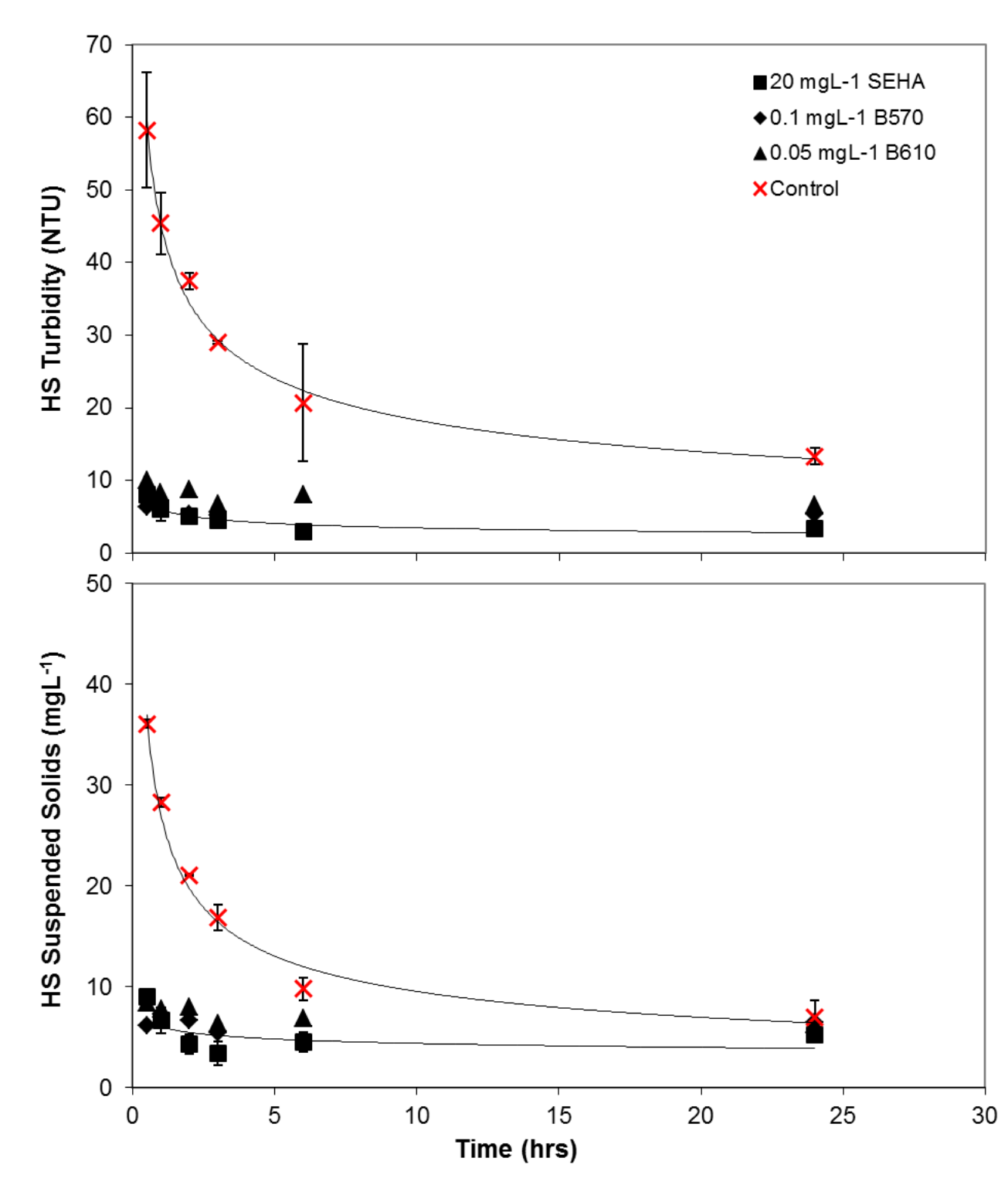

Figure 8-1: HS and polymer doses for cost benefit . Standard deviation error (n=2). 
Feasibility/Markets

The HS/polymer doses of $20 \mathrm{mg} / \mathrm{L}$ SEHA, $0.1 \mathrm{mg} / \mathrm{L}$ B570 and $0.05 \mathrm{mg} / \mathrm{L}$ B610 were selected for the cost: benefit analysis as they correspond to the optimal dose for turbidity and SS removal, and resulted in comparable supernatant water quality. The dose costs are shown in Table 8-1.

Table 8-1: Cost: benefit for polymer application

\begin{tabular}{ccccc}
\hline & $\begin{array}{c}\text { Product cost* } \\
\$ / \mathrm{kg}\end{array}$ & $\begin{array}{c}\text { Required dose } \\
\mathrm{kg} / 1000 \mathrm{~m}^{3}\end{array}$ & $\begin{array}{c}\text { Cost of dose } \\
\$ / 1000 \mathrm{~m}^{3}\end{array}$ & Performance factor \\
\hline SEHA & $1.5^{*}$ & 20 & 30 & 1 \\
B570 & $9.95^{* *}$ & 0.1 & 1.00 & 30 \\
B610 & $9.25^{* *}$ & 0.05 & 0.46 & 65 \\
\hline
\end{tabular}

* (Weber 2011)

*** (Fraser 2011)

The SEHA cost is the proposed Solid Energy New Zealand Ltd (SENZ) market price. The polymer prices were sourced by personal communication from Orica-Chemnet, a bulk chemical supplier to the mining industry

Table 8-1 shows the cost of treating $1000 \mathrm{~m}^{3}$ of AMD at a dose rate of $20 \mathrm{mg} / \mathrm{L}$ SEHA would be $\$ 30$. The same volume of AMD could be treated using lower doses of B570 and B610 at a cost of $\$ 1.00$ and $\$ 0.46$, respectively. Therefore, on a cost basis B570 and B610 are 30 and 65 times more efficient at removing residual SS from $\mathrm{CaCO}_{3}$ neutralised AMD than SEHA, per dollar spent.

This cost-benefit analysis does not assign any value to HS as a naturally occurring and ubiquitous material in West Coast streams. The residual HS from polymer dosing would not adversely affect the receiving environment, while the Crystalfloc polymers may. The environmental and sustainability value was not quantified in this study, though it may improve the overall cost: benefit performance SEHA.

Another important factor which may increase the value of HS is its maintained performance when under-dosed. The removal efficiency of HS remains high in underdosed scenarios while the Crystalfloc polymers underperform when under-dosed. Thus, field application of Crystalfloc would require a greater factor of safety than a HS system. 
Feasibility/Markets

\subsubsection{Cost: benefit of HS for Metal Removal}

To determine the cost: benefit of HS for metal removal, the cost: benefit of each HS dose condition for the BTP scenario was evaluated. The cost: benefit of metal removal for the Mangatini Stream-sump System (MSS) trials was not evaluated as no statistically significant HS enhanced metal removal was observed. The cost: benefit of Aldrich Humic Acid (AHA) dosed, Blackwater Treatment Plant (BTP) trials were not determined either as the AHA product is an expensive, commercial product, prohibiting application at the AMD treatment scale.

An operational cost: benefit analysis was used to show whether the additional cost of HS incorporation into AMD treatment enhanced the removal of the target metal group at the same rate as conventional neutralisation treatment. The addition of HS may increase the removal of a given metal compared to conventional treatment, but HS dosing is not considered beneficial if the additional cost of HS dosing produces a less than proportional benefit, in the cost: benefit analysis.

The cost: benefit (units of dose cost (\$) per metal removal (mg) - per litre of AMD) was evaluated as a function of metal (Me) removal and treatment cost, using Equation 8-1.

$$
\text { Cost: benefit }=\frac{A x+H S y}{(R-N)}
$$

Equation 8-1

Cost: benefit equation

Where, $\mathrm{A}$ is the Alkalinity dose required to neutralise to the final $\mathrm{pH}\left(\mathrm{mg} \mathrm{CaCO}_{3} / \mathrm{L}\right)$; $\mathrm{x}$ is the Alkalinity cost $\left(\$ / \mathrm{mg} \mathrm{CaCO}_{3}\right)$; HS is the HS dose added (mg HS/L); $\mathrm{y}$ is the HS cost (\$/mg HS);

$\mathrm{R}$ is the raw AMD metal concentration ( $\mathrm{mg} / \mathrm{L})$; and $\mathrm{N}$ is the neutralised AMD metal concentration $(\mathrm{mg} / \mathrm{L})$.

The alkalinity cost was determined using Equation 8-2.

$$
x=\frac{C m_{w}}{50 e_{q} e}
$$

Alkalinity cost equation

Where, $\mathrm{C}$ is the alkalinity reagent cost $(\$ / \mathrm{mg})$;

$\mathrm{m}_{\mathrm{w}}$ is the molecular weight of the alkalinity reagent $(\mathrm{mg} / \mathrm{mmol})$; $\mathrm{e}_{\mathrm{q}}$ is the equivalent charge of the alkalinity reagent (meq/mmol); 
Feasibility/Markets

$\mathrm{e}$ is the neutralisation efficiency of the alkalinity reagent (\%); and 50 is the mass per equivalence of $\mathrm{CaCO}_{3}(\mathrm{mg} / \mathrm{meq})$.

The major variable affecting the cost: benefit analysis was the treatment cost. Figure 8-2 separates the total cost of HS dosed AMD into HS and alkalinity components.
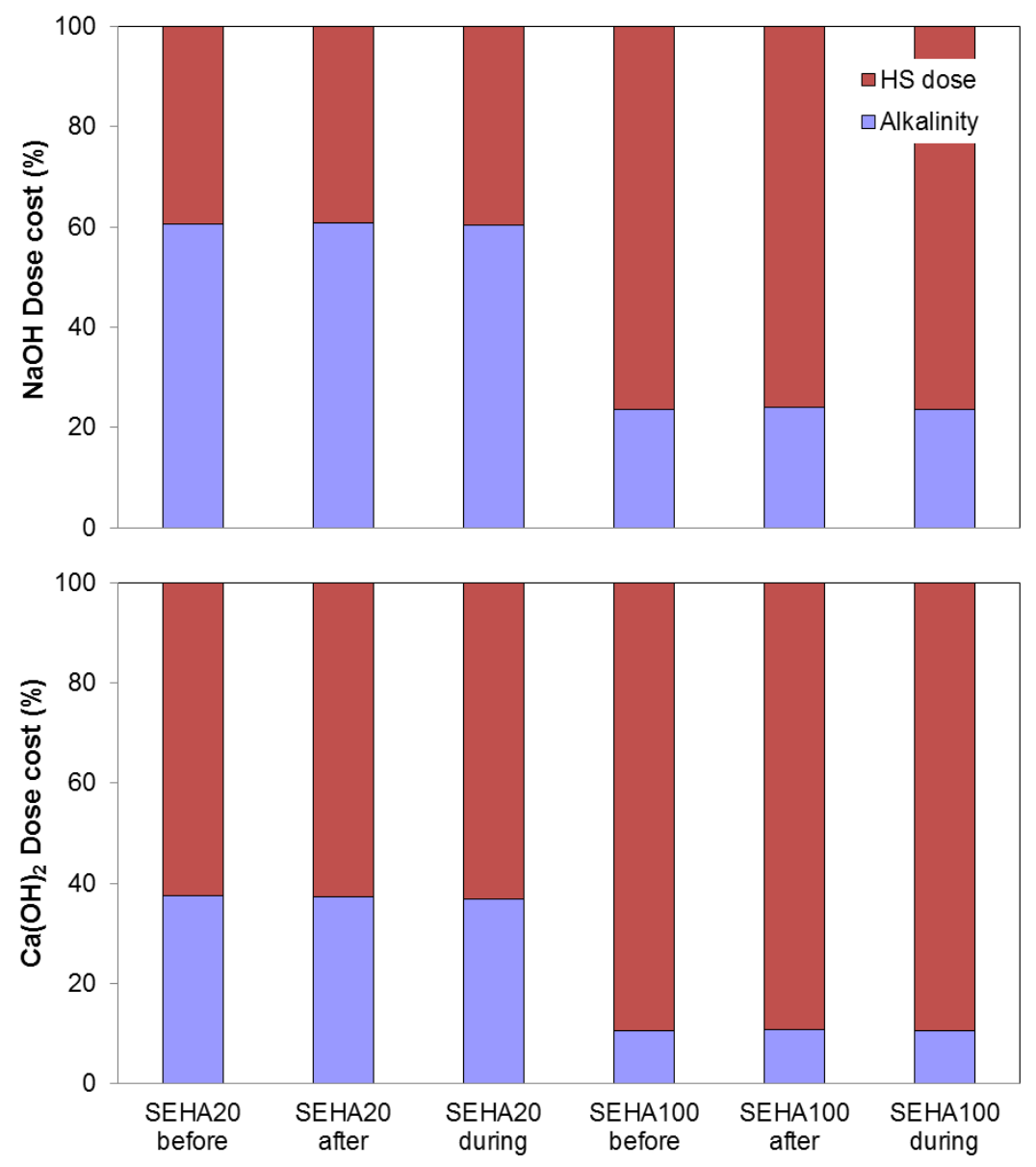

Figure 8-2: Distribution of HS dose cost

Figure 8-2 shows the interaction between the alkalinity and HS dose cost. The reagent cost of $\mathrm{NaOH}$ is relatively high and dominates the total cost $(61 \% \mathrm{NaOH})$ of SEHA20 dose conditions. However, the additional cost of $100 \mathrm{mg} / \mathrm{L}$ SEHA dose results in the HS component dominating the total cost $\left(76 \%\right.$ HS). The reagent cost of $\mathrm{Ca}(\mathrm{OH})_{2}$ is relatively low, resulting in the HS component of dosing dominating the total cost of SEHA20 (63\% HS) and SEHA100 (89\% HS). 
Feasibility/Markets

The results of the $\mathrm{NaOH}$ cost: benefit analysis are shown on Figure 8-3.
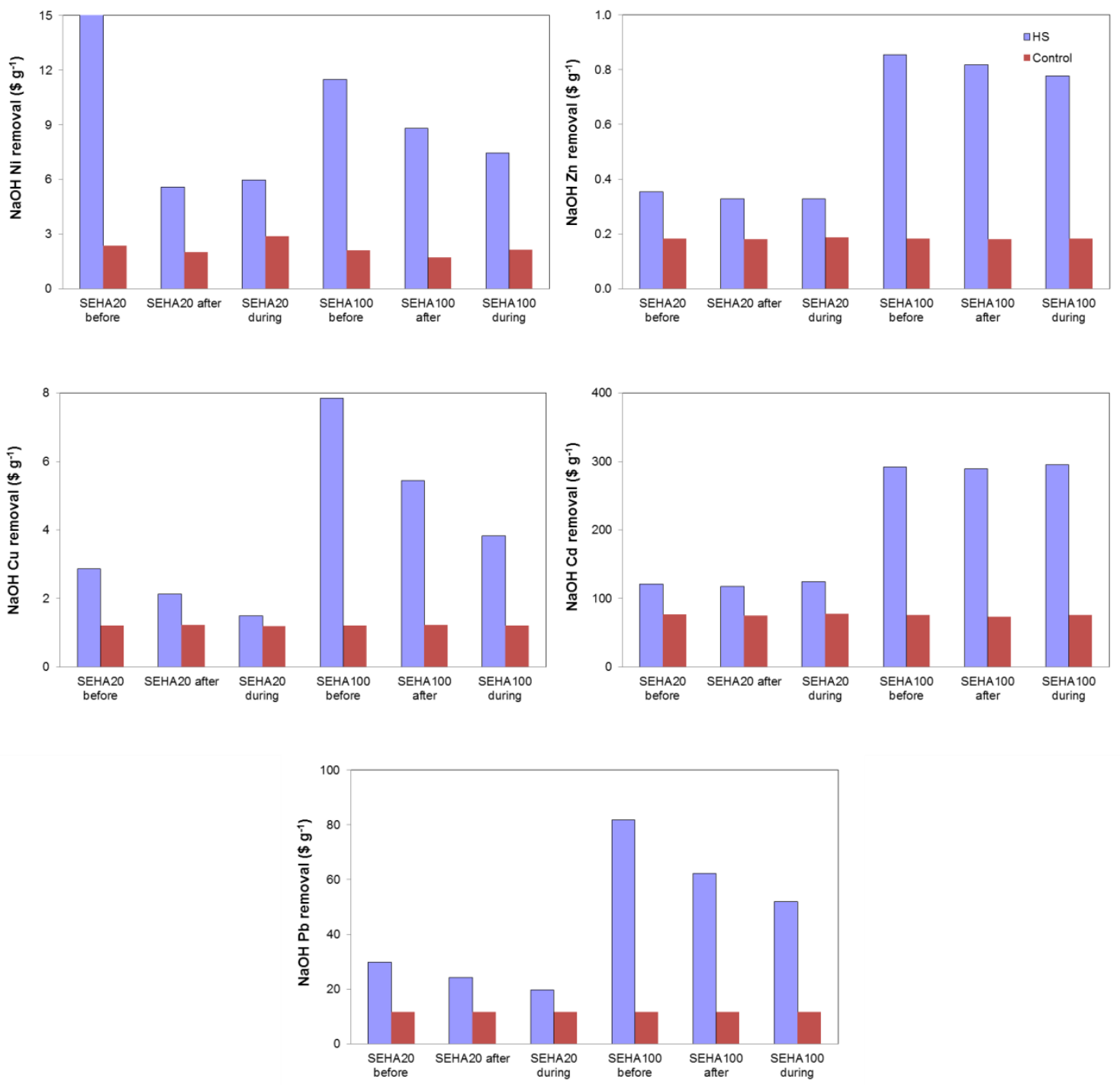

Figure 8-3: NaOH neutralised metal removal cost: benefit

NB: a lower cost per metal removal $\left(\$ \mathrm{~g}^{-1}\right)$ corresponds to a higher cost: benefit ratio.

Figure 8-3 shows that the cost: benefit ratio of HS dosing is lower than control conditions, suggesting HS dosing yields metal removal benefits at a lower cost efficiency than AMD neutralisation by $\mathrm{NaOH}$ alone. There is a general trend of SEHA20 HS dosing resulting in a higher cost: benefit than SEHA100 conditions, attributable to the lower HS cost of dosing with $20 \mathrm{mg} / \mathrm{L}$ SEHA.

Section 7.2.1.1 showed the removal of $\mathrm{Cu}$ was enhanced in the SEHA20 duringneutralisation condition. This is reflected on Figure 8-3 by a relatively high cost: benefit 
Feasibility/Markets

ratio. Table 8-2 shows the theoretical effluent metal concentrations that would be required from SEHA20 dosed AMD to maintain the cost: benefit efficiency of control samples.

Table 8-2: Required HS dosed metal concentrations (NaOH)

\begin{tabular}{ccccc}
\hline Metal & $\begin{array}{c}\text { Raw } \\
(\mathbf{m g} / \mathbf{L})\end{array}$ & $\begin{array}{c}\text { Control } \\
(\mathbf{m g} / \mathbf{L})\end{array}$ & $\begin{array}{c}\text { Alkalinity } \\
\text { removal rate } \\
(\mathbf{m g} / \mathbf{\$})\end{array}$ & $\begin{array}{c}\text { Required HS dosed } \\
\text { effluent conc. } \\
(\mathbf{m g} / \mathbf{L})\end{array}$ \\
\hline $\mathbf{N i}$ & 0.59 & 0.29 & 435 & 0.09 \\
$\mathbf{Z n}$ & 4.04 & 0.28 & 5405 & -2.15 \\
$\mathbf{C u}$ & 0.91 & 0.34 & 826 & -0.03 \\
$\mathbf{C d}$ & 0.011 & 0.002 & 13 & -0.004 \\
$\mathbf{P b}$ & 0.09 & 0.03 & 86 & -0.01 \\
\hline
\end{tabular}

Table 8-2 shows that it would in fact be impossible for the addition of $20 \mathrm{mg} / \mathrm{L} \mathrm{SEHA} \mathrm{to}$ result in the same $\mathrm{Zn}, \mathrm{Cu}, \mathrm{Cd}$ and $\mathrm{Pb}$ metal removal efficiency (per treatment cost) as alkalinity alone conditions, as the required HS dosed concentrations would be negative. For example, for the SEHA20 during-neutralisation by $\mathrm{NaOH}$ condition; neutralisation by $\mathrm{NaOH}$ removes $0.57 \mathrm{mg} / \mathrm{L} \mathrm{Cu}$ and represents $61 \%$ of the total dose cost. Addition of 20 $\mathrm{mg} / \mathrm{L}$ SEHA removes an additional removal of $0.19 \mathrm{mg} / \mathrm{L} \mathrm{Cu}$ for the remaining $39 \%$ of the total dose cost, at a lower metal removal efficiency (per treatment cost). To maintain the same metal removal efficiency as $\mathrm{NaOH}$ neutralisation alone, a final $\mathrm{Cu}$ concentration of $-0.03 \mathrm{mg} / \mathrm{L}$ would be required.

Theoretically, the addition of SEHA20 could result in an enhanced cost: benefit ratio for $\mathrm{Ni}$ if $\mathrm{HS}$ addition resulted in almost complete removal of $\mathrm{Ni}$, to below $0.09 \mathrm{mg} / \mathrm{L}$. However, section 7.2.1.1 showed that HS did not conclusively enhance the removal of Ni for any condition. 
Feasibility/Markets

Figure 8-4 shows the results of the $\mathrm{Ca}(\mathrm{OH})_{2}$ neutralised cost: benefit analysis.
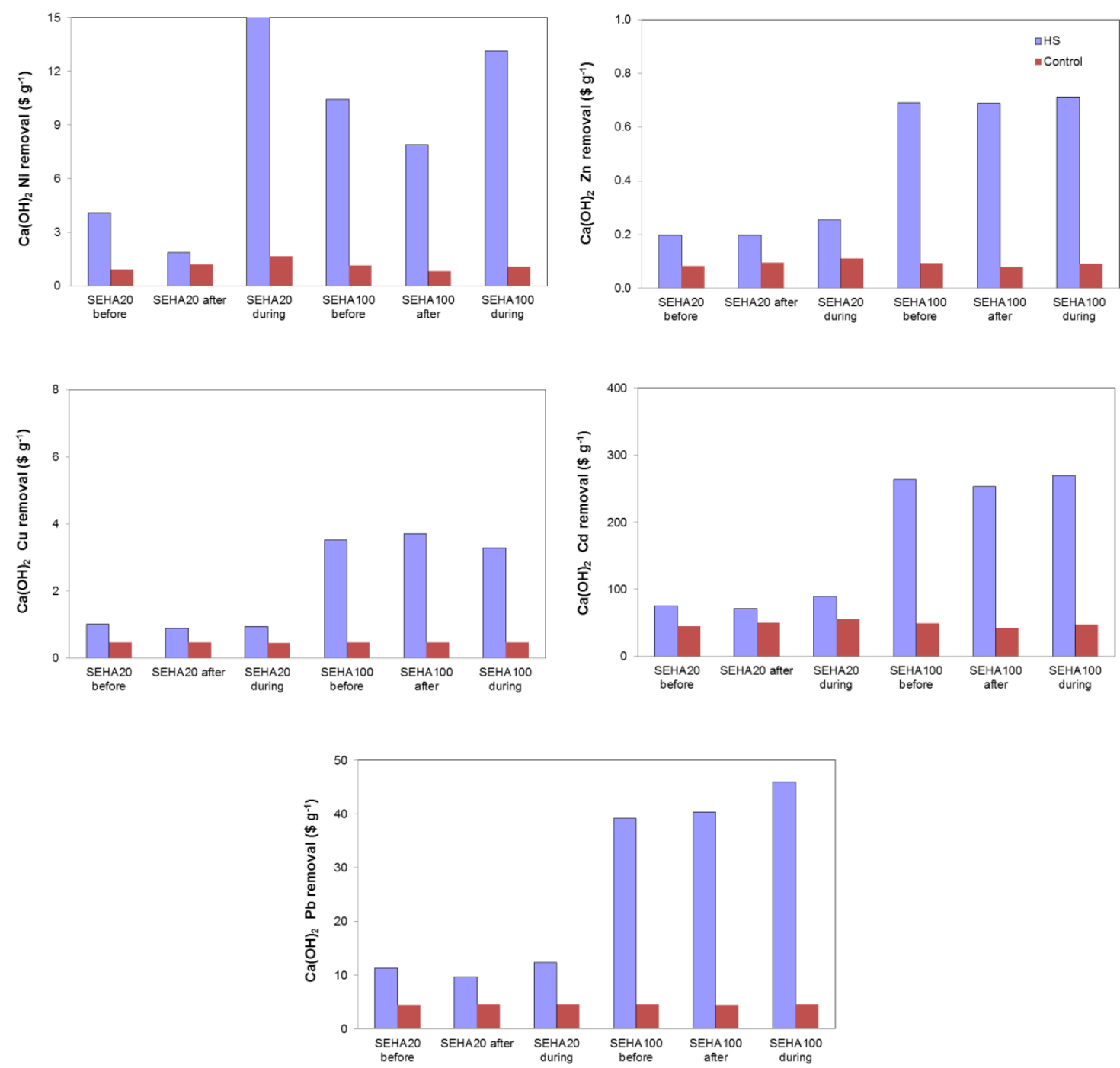

Figure 8-4: $\mathrm{Ca}(\mathrm{OH})_{2}$ neutralised metal removal cost: benefit

Figure 8-4 shows that again no HS dosed conditions resulted in a higher cost: benefit ratio than $\mathrm{Ca}(\mathrm{OH})_{2}$ neutralisation alone. The SEHA20 conditions for $\mathrm{Ni}, \mathrm{Zn}, \mathrm{Cd}, \mathrm{Pb}$ and particularly $\mathrm{Cu}$ were relatively low, but none outperform the control.

Despite slightly higher metal concentrations, the control cost: benefit ratio is higher for $\mathrm{Ca}(\mathrm{OH})_{2}$ neutralised AMD because $\mathrm{Ca}(\mathrm{OH})_{2}$ is cheaper than $\mathrm{NaOH}$. Table 8-3 shows the theoretical effluent metal concentrations that would be required from SEHA20 dosed AMD to maintain the cost: benefit of control samples. 
Feasibility/Markets

Table 8-3: Required HS dosed metal concentrations $\left(\mathrm{Ca}(\mathrm{OH})_{2}\right)$

\begin{tabular}{ccccc}
\hline Metal & $\begin{array}{c}\text { Raw } \\
(\mathbf{m g} / \mathbf{L})\end{array}$ & $\begin{array}{c}\text { Control } \\
(\mathbf{m g} / \mathbf{L})\end{array}$ & $\begin{array}{c}\text { Alkalinity removal } \\
\text { rate } \\
(\mathbf{m g} / \mathbf{\$})\end{array}$ & $\begin{array}{c}\text { Required HS dosed } \\
\text { effluent conc. } \\
(\mathbf{m g} / \mathbf{L})\end{array}$ \\
\hline $\mathbf{N i}$ & 0.59 & 0.32 & 1049 & -0.15 \\
$\mathbf{Z n}$ & 4.04 & 0.97 & 12011 & -4.43 \\
$\mathbf{C u}$ & 0.91 & 0.34 & 2248 & -0.67 \\
$\mathbf{C d}$ & 0.011 & 0.006 & 23 & -0.005 \\
$\mathbf{P b}$ & 0.09 & 0.03 & 234 & -0.08 \\
\hline
\end{tabular}

Table 8-3 shows that it would not be possible for HS addition to improve the cost: benefit ratio for any metal, as the required effluent metal concentrations were all negative. The HS component of the total cost is greater for HS dosed $\mathrm{Ca}(\mathrm{OH})_{2}$ neutralised conditions due to a lower alkalinity cost, as shown on Figure 8-2. The metal removal by HS would therefore have to be even greater for $\mathrm{Ca}(\mathrm{OH})_{2}$ conditions to decrease the cost: benefit ratio.

This section shows that the additional cost of SEHA dosing does not result in a proportional decrease in metal concentrations, compared to alkalinity neutralisation alone. Thus, a lower cost: benefit ratio was determined for all HS dose conditions by this analysis. The market price of SEHA would need to be decreased to around $\$ 500$ per tonne (from $\$ 1500 \mathrm{~T}^{-1}$ ) to get a similar cost: benefit as alkalinity neutralisation alone for removal of $\mathrm{Cu}$ and $\mathrm{Cd}$ from AMD.

This cost: benefit analysis was performed to indicate the feasibility of HS dosing in AMD neutralisation based on the results of this thesis. A more thorough investigation of cost: benefit may include:

1. Set lower target metal concentrations and investigate the cost of alkalinity dosing to achieve these concentrations - probably at $\mathrm{pH} 8-10$.

2. Assign non-linear value to metal removal to reflect the increased difficulty of removing metals which remain dissolved at circum-neutral $\mathrm{pH}$.

3. Incorporate the fixed costs of plant construction for HS manufacture (offsite) and dosing (onsite), in addition to variable costs of chemical dosage. 
Feasibility/Markets

4. Investigate the environmental value of HS as a renewable, sustainable and natural product and the potential of HS to reduce adverse impacts on the receiving environment, compared to commercial polymers.

Figure 7-4 shows the concentration of $\mathrm{Cu}$ is essentially constant over $\mathrm{pH} 6$ to 7 , which suggests no removal of $\mathrm{Cu}$ by adsorption or hydroxide precipitation over that $\mathrm{pH}$ range. The SEHA20 after-neutralisation condition reduced the residual $\mathrm{Cu}$ concentration by $66 \%$ (from 0.34 to $0.11 \mathrm{mg} / \mathrm{L}$ ), compared to the control. If the removal of $\mathrm{Cu}$ to $0.11 \mathrm{mg} / \mathrm{L}$ is not possible by neutralisation to any $\mathrm{pH}$, the value of $\mathrm{HS}$ removal of $\mathrm{Cu}$ shown by the SEHA20 after-neutralisation should be rated more highly. However, defining the value of the additional removal of $\mathrm{Cu}$ by $\mathrm{HS}$ would be subjective and is beyond the scope of this preliminary cost: benefit analysis. 


\subsection{Market Review}

This section discusses a few examples from the literature which use HS in agricultural applications to briefly identify other potential markets for SEHA worthy of further investigation.

\subsubsection{Agricultural Markets}

Humic substances play an important role in the yield and uptake of nutrients in agriculture. There is a lot of published and un-published evidence which supports the application of HS to agricultural land to enhance nutrient withholding capacity, fungi and bacteria abundance, fertiliser availability, detoxification of contaminants and salts, disease prevention and soil structure/texture.

Verlinden et al. (2009), (2010) investigated the application of liquid HS directly to soil and solid HS as a mineral fertiliser amendment. Both papers investigated the effect of HS on grass yields. The addition of HS (to low organic matter soils) increased the yield at the first grass cut by 3 to $16 \%$ for sandy loam soils and up to $66 \%$ for sand soils at HS dose rates between 6.4 and $8.3 \mathrm{~kg} \mathrm{HS} \mathrm{ha}^{-1}$ (Verlinden, Coussens et al. 2010). Addition of solid HS blended with mineral fertiliser resulted in the greatest dry matter production rates. While the addition of HS increased the yield at first cut, subsequent grass production was low, resulting in similar total dry matter production across HS amended and control plots (Verlinden, Pycke et al. 2009; Verlinden, Coussens et al. 2010). The authors identified the potential value of maximising the first cut dry matter yield with respect to silage production.

Verlinden et al. (2009) showed that application of HS increased the dry matter yield of potato crops by 13 to $17 \%$, at HS application rates between 3.9 and $8.3 \mathrm{~kg} \mathrm{ha}^{-1}$. The addition of HS also resulted in a significant increase in nitrate $(11-24 \%)$ and phosphate (54-60\%) uptake in plants grown in sand soils, which the authors linked to HS solubilisation of nutrients, while plants grown in loamy soils showed statistically indeterminate enhancement (Verlinden, Pycke et al. 2009). These trials were undertaken in calcareous soils where phosphate availability in particular may have limited potato yield in the control plots (Verlinden, Pycke et al. 2009; Seyedbagheri 2010).

Seyedbagheri (2010) showed that potato yields in calcareous soils increased linearly from 37.6 to $43.1 \mathrm{t} \mathrm{ha}^{-1}$ by increasing humic acid (HA) application from 0 to $2.2 \mathrm{~kg} \mathrm{ha}^{-1}$, above 
Feasibility/Markets

which the yield declined. Other trials showed no increase in HA amended potato yields grown in silt-clay-loam soils as extraction of clay bound nutrients by HA was insufficient to make additional nutrients available. Thus, Seyedbagheri (2010) concluded HS application in agriculture was variable due to the complex nature of HS and a lack of understanding of site specific soil total carbon, mineral and HS-mineral complex interactions with nutrients.

Humic substances improve livestock gastrointestinal tract health, nutrient adsorption and nutritional status (Wang, Chen et al. 2008). Humic substance supplementation of feed to maintain animal health has been investigated as an alternative to antibiotics and growth promoters (Ozturk, Ocak et al. 2010). Ozturk (2010) reported that broilers provided drinking water with $300 \mathrm{mg} / \mathrm{L}$ HS had a $4 \%$ higher daily weight gain and a $5 \%$ greater weight gain efficiency. Karaoglu et al. (2004) supplemented broiler feed with between 1000 and $3000 \mathrm{mg} / \mathrm{L} \mathrm{HS}$ and concluded that there was no statistically significant enhancement of weight gain or efficiency.

While the effects of HS supplementation of broiler feedstock may be statistically indeterminate over the ranges investigated, Wang et al. (2008) showed the addition of $10 \%$ HS to pig feed resulted in an increase in average daily growth of $11 \%$ and increased weight gain efficiency by $12 \%$.

\subsection{Summary - review}

An operational cost: benefit analysis for metal removal showed that no HS dosed conditions enhanced metal removal sufficiently to be economically feasible. The cost: benefit for the SEHA20 during-neutralisation by $\mathrm{NaOH}$ had an only slightly lower cost: benefit ratio than the control, but typically the high cost of HS dosing made an enhanced cost: benefit by HS dosing impossible, particularly for $100 \mathrm{mg} / \mathrm{L} \mathrm{SEHA} \mathrm{doses.}$ 


\section{Chapter 9. Conclusions}

The conclusions chapter is divided into two sections.

Section 1: General conclusions. This section draws together key aspects from the discussion of results as project conclusions. The general conclusions section complements the summary sections for each of the major results chapters; Chapter 4 to chapter 8 .

Section 2: Further work. This section describes how further work could address the limitations of this project and expand on the positive findings.

\subsection{General Conclusions}

Neutralisation of AMD by $\mathrm{NaOH}$ and $\mathrm{Ca}(\mathrm{OH})_{2}$ in the BTP scenario resulted in good water quality, with turbidity and suspended solid (SS) concentrations below detection limits. Addition of HS before $(\mathrm{pH} 2.8)$ and during $(\mathrm{pH} 4.5)$ neutralisation resulted in HS precipitation due to low solubility in acidic pH (Schnitzer and Kahn 1972). Precipitated HS then appeared to act as a nucleation site, resulting in HS incorporation into sludge, resulting in good water quality for both $\mathrm{NaOH}$ and $\mathrm{Ca}(\mathrm{OH})_{2}$ neutralised conditions.

Addition of $\mathrm{HS}$ after neutralisation $(\mathrm{pH} 7)$ by $\mathrm{NaOH}$ resulted in a significant increase in turbidity and SS of up to $24.4 \mathrm{NTU}$ and $18.4 \mathrm{mg} / \mathrm{L}$ for the SEHA100 condition, due primarily to dissolved HS. The absence of a multi-valent bridging cation (monovalent $\mathrm{Na}$ was dominant cation) was identified as critical in the lack of HS incorporation into AMD sludge. Conversely, addition of $\mathrm{HS}$ after neutralisation $(\mathrm{pH} 7)$ by $\mathrm{Ca}(\mathrm{OH})_{2}$ resulted in relatively low turbidity and SS of less than $2 \mathrm{NTU}$ and $5 \mathrm{mg} / \mathrm{L}$, respectively. The presence of divalent $\mathrm{Ca}(\sim 400 \mathrm{mg} / \mathrm{L})$ as a product of $\mathrm{Ca}(\mathrm{OH})_{2}$ dissociation facilitated inter-particle bridging of HS molecules (Chen and Elimelech 2007), resulting in the removal of a high degree of residual HS.

The low neutralisation efficiency of $\mathrm{CaCO}_{3}$ resulted in a high proportion of the $\mathrm{CaCO}_{3}$ dose remaining undissolved, which contributed to elevated turbidity and SS in control samples of $23.0 \mathrm{NTU}$ and $17.0 \mathrm{mg} / \mathrm{L}$. Thus, the water quality of $\mathrm{CaCO}_{3}$ neutralised AMD was controlled by the sedimentation of $\mathrm{CaCO}_{3}$ particulate; not precipitated metal hydroxides. When added after neutralisation, $\mathrm{HS}$ acted as a polymer adsorbing $\mathrm{CaCO}_{3}$ 
Conclusions

particulate (Lee, Elzinga et al. 2005), with the SEHA100-after condition reducing SS to $4.1 \mathrm{mg} / \mathrm{L}$ after 2 hours sedimentation.

The performance of SEHA as a polymer with respect to removal of residual $\mathrm{CaCO}_{3} \mathrm{SS}$ was investigated further. A dose of 20mg/L SEHA resulted in SS of $9 \mathrm{mg} / \mathrm{L}$ after $30 \mathrm{~min}$ sedimentation. Two commercially available polymers, Crystalfloc B570 and B610 achieved similar water quality at dose rates of 0.05 and $0.1 \mathrm{mg} / \mathrm{L}$, respectively. A cost: benefit analysis showed that polymers were 30-65 times more efficient (on a dose: cost basis) at removal of residual $\mathrm{CaCO}_{3}$ than SEHA.

The concentration of metals in neutralised AMD varied depending on which alkalinity reagent was used. Neutralisation of AMD using $\mathrm{NaOH}$ resulted in a decreased concentration for all metals in the target group. The addition of HS did not enhance the removal of $\mathrm{Ni}, \mathrm{Zn}$ or $\mathrm{Pb}$, while data of $\mathrm{Cd}$ were indeterminate. The SEHA20 and SEHA100 during-neutralisation conditions enhanced the removal of $\mathrm{Cu}$ by over $50 \%$. The high removal of $\mathrm{Cu}$ was attributed to a high affinity of $\mathrm{Cu}$ for HS (Čežíková, Kozler et al. 2001; Milne, Kinniburgh et al. 2003; Pehlivan and Arslan 2007), particularly phenolic reactive sites (shown by equilibrium modelling) which were shown to be well represented on SEHA by HS characterisation. Analysis of the SEHA100 after-neutralisation by $\mathrm{NaOH}$ condition suggested that HS complexed metals in the dissolved phase $(<0.45 \mu \mathrm{m})$ would not facilitate contaminant transport as metal concentrations did not spike in the highly turbid sample.

Neutralisation of $\mathrm{AMD}$ by $\mathrm{Ca}(\mathrm{OH})_{2}$ resulted in slightly higher control metal concentrations than $\mathrm{NaOH}$, possibly due to $\mathrm{Ca}$ competition for sorption sites on precipitated $\mathrm{Fe} / \mathrm{Al}$ hydroxides. The effects of HS dosing on metal removal were therefore more pronounced, although $\mathrm{Ni}$ and $\mathrm{Pb}$ removal was not enhanced. The SEHA20 duringand after-neutralisation conditions enhanced the removal of $\mathrm{Cu}$ by 55 and $66 \%$, respectively. The Cd removal was also enhanced for all HS dose conditions, with $80 \%$ removal by SEHA20 after-neutralisation, due to a moderately high Cd affinity for HS (Čežíková, Kozler et al. 2001; Milne, Kinniburgh et al. 2003). Equilibrium modelling showed that $\mathrm{Cd}$ was less selective, binding to both carboxylic and phenolic reactive groups. The $\mathrm{Ca}(\mathrm{OH})_{2}$ results suggested the removal of $\mathrm{Zn}$ was enhanced by HS dosing, but the validity of these results is questionable as both the literature review and speciation modelling showed that $\mathrm{Zn}$ had a low affinity for HS complexation. 


\section{Conclusions}

Neutralisation of $\mathrm{AMD}$ by $\mathrm{CaCO}_{3}$ resulted in higher concentrations of $\mathrm{Ni}, \mathrm{Zn}$ and $\mathrm{Pb}$; but lower concentrations of $\mathrm{Cu}$ and $\mathrm{Cd}$, compared to $\mathrm{Ca}(\mathrm{OH})_{2}$ neutralisation. Due to the low dissolution of $\mathrm{CaCO}_{3}$, the dominant particulate species was calcite $\left(\mathrm{CaCO}_{3}\right)$. Thus, the adsorption affinities of the target metals for calcite dominated the removal of metals by adsorption. Both $\mathrm{Cu}$ and $\mathrm{Cd}$ had high calcite binding affinities and thus were present at a lower concentration in control samples (García-Sánchez and Álvarez-Ayuso 2002). Subsequent HS dosing resulted in up to $40 \%$ additional removal of $\mathrm{Cd}$ for the duringneutralisation condition, while the removal of other metals was not enhanced by HS dosing.

The addition of $\mathrm{HS}$ was expected to enhance $\mathrm{Pb}$ removal as $\mathrm{Pb}$ has a high complexation affinity for both HS and precipitated metal hydroxides (Čežíková, Kozler et al. 2001; Lee, Bigham et al. 2002; Milne, Kinniburgh et al. 2003; Pehlivan and Arslan 2007). The concentrations of $\mathrm{Pb}$ in the raw AMD were only four times the detection limit, which was relatively high at $0.024 \mathrm{mg} / \mathrm{L}$. Thus, any interaction between $\mathrm{Pb}$ and $\mathrm{HS}$ or metal hydroxide was difficult to identify. The addition of $\mathrm{HS}$ showed no Ni removal enhancement which is supported by the literature review and speciation modelling which state Ni had a low complexation affinity for HS (Čežíková, Kozler et al. 2001; Pehlivan and Arslan 2007).

An operational cost: benefit analysis for metal removal showed that none of the HS dosed conditions investigated in this thesis enhanced metal removal sufficiently to be economically feasible. The cost: benefit for the SEHA20 during-neutralisation by $\mathrm{NaOH}$ had an only slightly lower cost: benefit ratio than the control, but typically the high cost of HS dosing makes an enhanced cost: benefit by HS dosing impossible. 


\subsection{Further work}

This section describes how further work could address the limitations of this project and expand on the positive findings.

The raw AMD used in these experiments was stored for up to 12 months before being used in the lab, resulting in some decay in metal concentrations which may have affected the results. The AMD was not replenished regularly as the variability of AMD at the Stockton Mine would make comparison of results from different batches difficult.

Field trials may give a better indication of the capacity for HS enhanced neutralisation of AMD. The performance of HS over a range of different acidity and metal concentrations was not investigated in this project. Background SS (comprised of disturbed sediments and coal fines), which ranges onsite from 15-25 mg/L (Davies, Weber et al. 2011b), was negligible in lab trials due to the long settlement time during storage. The inclusion of background SS in field trials may affect the settleability of control and HS dosed floc.

The variability in $\mathrm{pH}$ between different HS dose conditions meant HS enhanced metal removal had to be compared to control standards. This was a limitation as HS dosed metal removal could not be directly compared between HS dose conditions, as the proportion of metal removed by hydroxide precipitation/adsorption/HS complexation would vary with $\mathrm{pH}$. Thus, the HS complexation conditions were slightly different for each HS dose condition.

The operational cost: benefit analysis is limited in that it does not consider the additional value of metal removal by HS to concentrations lower than conventional neutralisation can achieve. Thus, a more favourable cost: benefit analysis may show HS dosing as more economically feasible.

This project could be improved upon by trialling HS incorporation into AMD neutralisation over a greater range of $\mathrm{pH}$. The $\mathrm{PZC}$ of most aluminium and iron oxides ranges from pH 6.5 to 8.5 (Evangelou 1998; Sparks 2003), while the dissociation constant of carboxylic groups on HS is around 3 (Milne, Kinniburgh et al. 2001). Thus, HS complexation of metals may be more effective at lower $\mathrm{pH}$, where competition for removal from adsorption processes would be less.

The metal removal results showed that for some scenarios, removal of $\mathrm{Cu}$ and $\mathrm{Cd}$ was approximately equal for 20 and $100 \mathrm{mg} / \mathrm{L}$ SEHA doses. Trials at much lower HS doses 


\section{Conclusions}

may therefore result in good metal removal at a lower HS dose cost, proving HS enhancement economically feasible.

Further work using alkali extracted HS for removal of dissolved metals from wastewaters

should focus on contaminated waters with high concentrations of $\mathrm{Cu}, \mathrm{Pb}$ and $\mathrm{Cd}$. The high complexation affinity of HS for these metals may result in economically feasible metal removal for specialised, highly contaminated waters. 



\section{Chapter 10. References}

Al-Reasi, H. A., C. M. Wood and D. S. Smith (2011). "Physicochemical and spectroscopic properties of natural organic matter (NOM) from various sources and implications for ameliorative effects on metal toxicity to aquatic biota." Aquatic Toxicology 103(3-4): 179-190.

American Public Health Association (APHA) (2005). Standard methods for the examination of water \& wastewater. Washington D.C.

American Society for Testing and Materials (ASTM) (2008). Standard Practice for CoagulationFlocculation Jar Test of Water. West Conshohocken, PA. D 2035.

Australian and New Zealand Environment and Conservation Council (ANZECC) and Agriculture and Resource Management Council of Australia and New Zealand (ARMCANZ) (2000). Australian and New Zealand Guidelines for Fresh and Marine Water Quality. Canberra.

Black, A., D. Trumm and P. Lindsay (2005). Impacts of Coal Mining on Water Quality and Metal Mobilisation: Case Studies from West Coast and Otago. Metal Contaminants in New Zealand. T. A. Moore, A. Black, J. J. Centeno, J. S. Harding and D. Trumm. Christchurch, resolutionz press.

Čežíková, J., J. Kozler, L. Madronová, J. r. Novák and P. Janoš (2001). "Humic acids from coals of the North-Bohemian coal field: II. Metal-binding capacity under static conditions." Reactive and Functional Polymers 47(2): 111-118.

Chen, J., B. Gu, E. J. LeBoeuf, H. Pan and S. Dai (2002). "Spectroscopic characterization of the structural and functional properties of natural organic matter fractions." Chemosphere 48(1): 59-68.

Chen, K. L. and M. Elimelech (2007). "Influence of humic acid on the aggregation kinetics of fullerene (C60) nanoparticles in monovalent and divalent electrolyte solutions." Journal of Colloid and Interface Science 309(1): 126-134. 
References

Christl, I., C. J. Milne, D. G. Kinniburgh and R. Kretzschmar (2001). "Relating ion binding by fulvic and humic acids to chemical composition and molecular size. 2. Metal binding." Environmental Science and Technology 35(Compendex): 2512-2517.

Collier, K. J. (1989). "Impacts of Wetland Afforestation on the Distribution of Benthic Invertebrates in Acid Streams of Westland, New Zealand." New Zealand Journal of Marine and Freshwater Research 23: 479-490.

Collier, K. J., O. J. Ball, A. K. Graesser, M. R. Main and M. J. Winterbourn (1990). "Do Organic and Anthropogenic Acidity Have Similar Effects on Aquatic Fauna?" Oikos 59(1): 33-38.

Collier, K. J. and M. J. Winterbourn (1987). "Faunal and chemical dynamics of some acid and alkaline New Zealand streams." Freshwater Biology 18(2): 227-240.

Crawford, R. J., I. H. Harding and D. E. Mainwaring (1993). "Adsorption and coprecipitation of single heavy metal ions onto the hydrated oxides of iron and chromium." Langmuir 9(11): 3050-3056.

CRC (1976). CRC Handbook of Chemistry \& Physics. Cleveland Ohio, CRC Press.

Davies, H., P. Weber, P. Lindsay, D. Craw, B. Peake and J. Pope (2011a). "Geochemical changes during neutralisation of acid mine drainage in a dynamic mountain stream, New Zealand." Applied Geochemistry 26(12): 2121-2133.

Davies, H., P. Weber, P. Lindsay, D. Craw and J. Pope (2011b). "Characterisation of acid mine drainage in a high rainfall mountain environment, New Zealand." Science of the Total Environment 409(15): 2971-2980.

Evangelou, V. P. (1995). Pyrite Oxidation and its Control. Lexington, University of Kentucky, CRC Press.

Evangelou, V. P. (1998). Environmental Soil and Water Chemistry. Lexington, University of Kentucky, John Wiley \& Sons, Inc.

Finkelman, R. B. (2005). Sources and Health Effects of Metals and Trace Elements in Our Environment: An Overview. Metal Contaminants in New Zealand. T. A. Moore, A. Black, J. J. Centeno, J. S. Harding and D. Trumm. Christchurch, resolutions press. 


\section{References}

Forstner, U., G. T. W. Wittmann, F. Prosi and J. H. van Lierde (1981). Metal Pollution in the Aquatic Environment. Heidelberg

Pretoria, Springer-Verlag.

Fraser, R. (2011). Alkalinity and Crystalfloc cost. W. Olds.

Gao, K., J. Pearce, J. Jones and C. Taylor (1999). "Interaction between peat, humic acid and aqueous metal ions." Environmental Geochemistry and Health 21(1): 13-26.

García-Sánchez, A. and E. Álvarez-Ayuso (2002). "Sorption of Zn, Cd and Cr on calcite. Application to purification of industrial wastewaters." Minerals Engineering 15(7): 539-547.

Greig, H. S., D. K. Niyogi, K. L. Hogsden, P. G. Jellyman and J. S. Harding (2010). "Heavy metals: confounding factors in the response of New Zealand freshwater fish assemblages to natural and anthropogenic acidity." Science of the Total Environment 408(16): 32403250.

Gundersen, D., S. Bustaman, W. Seim and L. Curtis (1994). "pH, Hardness and Humic Acid Influence Aluminium Toxicity to Rainbow Trout (Oncorhynchus mykiss) in Weakly Alkaline Waters." Canadian Journal of Fisheries and Aquatic Sciences $\mathbf{5 1 .}$

Gustafsson, J. P. (2010). Visual MINTEQ, Version 3.0.

Harbrow, M. (2001). Ecology of Streams Affected by Acid Mine Drainage near Westport, South Island, New Zealand. School of Biological Sciences. Christchurch, University of Canterbury. Master of Science.

Harding, J. S. (2005). Impacts of Metals and Mining on Stream Communities. Metal Contaminants in New Zealand. T. A. Moore, A. Black, J. J. Centeno, J. S. Harding and D. Trumm. Christchurch, resolutionz press.

Harding, J. S. and I. Boothroyd (2004). Impacts of Mining. Freshwaters of New Zealand. J. S. Harding, P. Mosley, P. Charles and B. Sorrell. Christchurch, New Zealand Hydrological Society INC.

Havelcová, M., J. Mizera, I. Sýkorová and M. Pekař (2009). "Sorption of metal ions on lignite and the derived humic substances." Journal of Hazardous Materials 161(1): 559-564. 
References

Herrmann, R. and I. Baumgartner (1992). "Aluminium species distribution during mixing of acid coal and slate mine drainage with neutral stream waters."

Holmes, P. R. and F. K. Crundwell (2000). "The kinetics of the oxidation of pyrite by ferric ions and dissolved oxygen: an electrochemical study." Geochimica et Cosmochimica Acta 64(2): 263-274.

Hyne, R. V., F. Pablo, M. Julli and S. J. Markich (2005). "Influence of water chemistry on the acute toxicity of copper and zinc to the cladoceran Ceriodaphnia of dubia." Environmental Toxicology and Chemistry 24(7): 1667-1675.

International Organisation for Standardisation (1999). ISO 5073: Brown Coals and Lignites Determination of Humic Acid.

Jochová, M., M. Punčochář, J. Horáček, K. Štamberg and D. Vopálka (2004). "Removal of heavy metals from water by lignite-based sorbents." Fuel 83(9): 1197-1203.

Johnston, C. T. and Y. O. Aochi (1996). Fourier Transform Infrared and Raman Spectroscopy. Methods of Soil Analysis. Part 3. Chemical Methods. D. L. Sparks. Madison, WI, Soil Science Society of America, Inc.

Karaoglu, M., M. Macit, N. Esenbuga, H. Durbag, O. Bilgin and L. Turgut (2004). "Effect of Supplemental Humate at Different Levels on the Growth Performance, Slaughter and Carcass Traits of Broilers." International Journal of Poultry Science 34: 406-410.

Kelly, M. (1988). Mining and the Freshwater Environment. Durham, UK, Elsevier Applied Science.

Kholodov, V. A., A. N. Kovalenko, N. A. Kulikova, G. F. Levedeva and I. V. Perminova (2004). Enhanced Detoxifying Ability of Hydroquinones-Enriched Humic Derivatives with Respect to Copper. Humic Substances and Soil and Water Environment, San Pedro, San Paulo, Brazil.

Kretzschmar, R. and H. Sticher (1997). "Transport of Humic-Coated Iron Oxide Colloids in a Sandy Soil: Influence of Ca2+ and Trace Metals." Environmental Science \& Technology 31(12): 3497-3504.

Kurková, M., Z. Klika, C. Kliková and J. Havel (2004). "Humic acids from oxidized coals: I. Elemental composition, titration curves, heavy metals in HA samples, nuclear magnetic resonance spectra of HAs and infrared spectroscopy." Chemosphere 54(8): 1237-1245. 


\section{References}

Lakshtanov, L. Z. and S. L. S. Stipp (2007). "Experimental study of nickel(II) interaction with calcite: Adsorption and coprecipitation." Geochimica et Cosmochimica Acta 71(15): 3686-3697.

Lee, G., J. M. Bigham and G. Faure (2002). "Removal of trace metals by coprecipitation with Fe, $\mathrm{Al}$ and $\mathrm{Mn}$ from natural waters contaminated with acid mine drainage in the Ducktown Mining District, Tennessee." Applied Geochemistry 17(5): 569-581.

Lee, Y. J., E. J. Elzinga and R. J. Reeder (2005). "Cu(II) adsorption at the calcite-water interface in the presence of natural organic matter: Kinetic studies and molecular-scale characterization." Geochimica et Cosmochimica Acta 69(1): 49-61.

Linnik, P. N. and T. A. Vasilchuk (2005). Role of Humic Substances in the Complexation and Detoxification of Heavy Metals: Case Study of the Dnieper Reservoirs. Use of Humic Substances to Remediate Polluted Environments: From Theory to Practice. I. V. Perminova, K. Hatfield and N. Hertkorn. Dordrecht, The Netherlands, Springer. 52.

Lottermoser, B. G. (2003). Mine Wastes: Characterization, Treatment and Environmental Impacts. Cairns, Australia, Springer.

McCauley, C. A., A. D. O'Sullivan, P. A. Weber and D. Trumm (2010). "Variability of Stockton Coal Mine drainage chemistry and its treatment potential with biogeochemical reactors." New Zealand Journal of Geology and Geophysics 53(2-3): 211-226.

Meador, J. P. (1991). "The interaction of $\mathrm{pH}$, dissolved organic carbon, and total copper in the determination of ionic copper and toxicity." Aquatic Toxicology 19(1): 13-31.

Milne, C. J., D. G. Kinniburgh and E. Tipping (2001). "Generic NICA-Donnan model parameters for proton binding by humic substances." Environmental Science and Technology 35(Compendex): 2049-2059.

Milne, C. J., D. G. Kinniburgh, W. H. Van Riemsdijk and E. Tipping (2003). "Generic NICA - Donnan model parameters for metal-ion binding by humic substances." Environmental Science and Technology 37(Compendex): 958-971.

Monteil-Rivera, F., E. B. Brouwer, S. Masset, Y. Deslandes and J. Dumonceau (2000). "Combination of X-ray photoelectron and solid-state $13 \mathrm{C}$ nuclear magnetic resonance 
References

spectroscopy in the structural characterisation of humic acids." Analytica Chimica Acta 424(2): 243-255.

Moore, T. A., L. Zhongsheng, C. M. Nelson, R. B. Finkelman and R. Boyd (2005). Concentration of Trace Elements in Coal Beds. Metal Contaminants in New Zealand. T. A. Moore, A. Black, J. J. Centeno, J. S. Harding and D. Trumm, Resolutionz Press.

Morel, F. (1983). Principles of Aquatic Chemistry, Wiley.

Moses, C. O., D. Kirk Nordstrom, J. S. Herman and A. L. Mills (1987). "Aqueous pyrite oxidation by dissolved oxygen and by ferric iron." Geochimica et Cosmochimica Acta 51(6): 15611571.

Novak, J., J. Kozler, P. Jano, J. Cezikova, V. Tokarova and L. Madronova (2001). "Humic acids from coals of the North-Bohemian coal field - I. Preparation and characterization." Reactive and Functional Polymers 47(2): 101-109.

O'Halloran, K., J.-A. Cavanagh and J. S. Harding (2008). "Response of a new zealand mayfly (Deleatidium spp.) to acid mine drainage: Implications for mine remediation." Environmental Toxicology and Chemistry 27(5): 1135-1140.

O'Sullivan, A. D. (2005). Passive Treatment Technologies for Managing Metal Mine Wastes: Lessons Learnt from Global Applications. Metal Contaminants in New Zealand. T. A. Moore, A. Black, J. J. Centeno, J. S. Harding and D. Trumm. Christchurch, revolutionz press.

Ozturk, E., N. Ocak, I. Coskun, S. Turhan and G. Erener (2010). "Effects of humic substances supplementation provided through drinking water on performance, carcass traits and meat quality of broilers." Journal of Animal Physiology and Animal Nutrition 94(1): 7885.

Pehlivan, E. and G. Arslan (2007). "Removal of metal ions using lignite in aqueous solution-Low cost biosorbents." Fuel Processing Technology 88(1): 99-106.

Perminova, I. V. and K. Hatfield (2005). Remediation Chemistry of Humic Substances: Theory and Implications for Technology. Use of Humic Substances to Remediate Polluted Environments: From Theory to Practice, Springer. 


\section{References}

Pope, J., N. Newman, D. Craw, D. Trumm and R. Rait (2010a). "Factors that influence coal mine drainage chemistry West Coast, South Island, New Zealand." New Zealand Journal of Geology and Geophysics 53(2-3): 115-128.

Pope, J., P. Weber, A. Mackenzie, N. Newman and R. Rait (2010b). "Correlation of acid base accounting characteristics with the Geology of commonly mined coal measures, West Coast and Southland, New Zealand." New Zealand Journal of Geology and Geophysics 53(2-3): 153-166.

Rouff, A. A., E. J. Elzinga, R. J. Reeder and N. S. Fisher (2005). "The influence of pH on the kinetics, reversibility and mechanisms of $\mathrm{Pb}$ (II) sorption at the calcite-water interface." Geochimica et Cosmochimica Acta 69(22): 5173-5186.

Ryan, A. C., E. J. Van Genderen, J. R. Tomasso and S. J. Klaine (2004). "Influence of natural organic matter source on copper toxicity to larval fathead minnows (Pimephales promelas): Implications for the biotic ligand model." Environmental Toxicology and Chemistry 23(6): 1567-1574.

Salati, S., F. Adani, C. Cosentino and G. Torri (2008). "Studying soil organic matter using 13C CPMAS NMR: The effect of soil chemical pre-treatments on spectra quality and representativity." Chemosphere 70(11): 2092-2098.

Schnitzer, M. and E. H. Hansen (1970). "Organo-metallic Interactions in Soils: An Evaluation of Methods for the Determination of Stability Constants of Metal-fulvic Acid Complexes." Soil Science 109(6): 333-340.

Schnitzer, M. and S. U. Kahn (1972). Humic Substances in the Environment. New York, Dekker.

Schulten, H. R. and M. Schnitzer (1993). "A state of the art structural concept for humic substances." Naturwissenschaften 80(1): 29-30.

Schwartz, M. L., P. J. Curtis and R. C. Playle (2004). "Influence of natural organic matter source on acute copper, lead, and cadmium toxicity to rainbow trout (Oncorhynchus mykiss)." Environmental Toxicology and Chemistry 23(12): 2889-2899.

Seyedbagheri, M.-M. (2010). "Influence of Humic Products on Soil Health and Potato Production." Potato Research 53(4): 341-349. 
References

Skousen, J. G., A. Sexton and P. F. Ziemkiewicz (2000). Acid Mine Drainage Control and Treatment. Reclamation of Drastically Disturbed Lands.

SNF FLOERGER Technical Publication: Water Soluble Polymers. Riceboro, GA.

Snoeyink, V. L. and D. Jenkins (1980). Water Chemistry, John Wiley \& Sons.

Solid Energy New Zealand Limited (SENZ). (2011). "Industry History: Buller." from http://www.coalnz.com/index.cfm/1,194,0,49,html.

Somerfield, P. J., J. Michael Gee and R. M. Warwick (1994). "Benthic community structure in relation to an instantaneous discharge of waste water from a tin mine." Marine Pollution Bulletin 28(6): 363-369.

Sparks, D. L. (2003). Environmental Soil Chemistry. Newark, Delaware, Academic Press.

Stevenson, F. J. (1994). Humus Chemistry: Genesis, Composition, Reactions, John Wiley \& Sons, Inc.

Swift, R. S. (1996). Organic Matter Characterization. Methods of Soil Analysis. Part 3. Chemical Methods. D. L. Sparks. Madison, WI, Soil Science Society of America, Inc.

Tchobanoglous, G., F. Burton, H. D. Stensel and M. Eddy (2003). Wastewater Engineering: Treatment and Reuse, McGraw-Hill.

Tipping, E. (2002). Cation Binding by Humic Substances. Cambridge, UK, Cambridge University Press.

Trumm, D. (2010). "Selection of active and passive treatment systems for AMD-flow charts for New Zealand conditions." New Zealand Journal of Geology and Geophysics 53(2-3): 195210.

Trumm, D. A., M. Watts and P. Gunn (2006). AMD Treatment in New Zealand - Use of Small-scale Passive Systems. International Conference on Acid Rock Drainage (ICARD), St. Louis MO, American Society of Mining and Reclamation.

Verlinden, G., T. Coussens, A. De Vliegher, G. Baert and G. Haesaert (2010). "Effect of humic substances on nutrient uptake by herbage and on production and nutritive value of herbage from sown grass pastures." Grass and Forage Science 65(1): 133-144. 


\section{References}

Verlinden, G., B. Pycke, J. Mertens, F. Debersaques, K. Verheyen, G. Baert, J. Bries and G. Haesaert (2009). "Application of Humic Substances Results in Consistent Increases in Crop Yield and Nutrient Uptake." Journal of Plant Nutrition 32(9): 1407-1426.

Wang, Q., Y. J. Chen, J. S. Yoo, H. J. Kim, J. H. Cho and I. H. Kim (2008). "Effects of supplemental humic substances on growth performance, blood characteristics and meat quality in finishing pigs." Livestock Science 117(2-3): 270-274.

Ward, C. R. (2002). "Analysis and significance of mineral matter in coal seams." International Journal of Coal Geology 50(1-4): 135-168.

Warren, L. A. and A. P. Zimmerman (1994). "Suspended Particulate Oxides and Organic Matter Interactions in Trace Metal Sorption Reactions in a Small Urban River." Biogeochemistry 24(1): 21-34.

Watzlaf, G. R., K. T. Schroder, R. L. P. Kleinmann, C. L. Kairies and R. W. Nairn (2004). The Passive Treatment of Coal Mine Drainage, U.S. Department of Energy, University of Oklahoma.

Weber, P. (2011). Alkalinity and SEHA cost. W. Olds.

Weber, W. J., J. Tang and Q. Huang (2006). "Development of Engineered Natural Organic Sorbents for Environmental Applications. 1. Materials, Approaches, and Characterizations." Environmental Science \& Technology 40(5): 1650-1656.

Webster, J. G. (1995). "Chemical Processes Affecting Trace Metal Transport in the Waihou River and Estuary, New Zealand." New Zealand Journal of Marine and Freshwater Research 29: 539-553.

Webster, J. G., P. J. Swedlund and K. S. Webster (1998). "Trace Metal Adsorption onto an Acid Mine Drainage Iron(III) Oxy Hydroxy Sulfate." Environmental Science \& Technology 32(10): 1361-1368.

Weisener, C. G. and P. A. Weber (2010). "Preferential oxidation of pyrite as a function of morphology and relict texture." New Zealand Journal of Geology and Geophysics 53(2-3): 167-176.

Whatman International Limited (1999). Laboratory Product Guide. 
References

Wilbur, S. (2007). Performance Characteristics of the Agilent 7500cx. A. T. INC. Bellevue, WA, Agilent Technologies.

Williamson, M. A. and J. D. Rimstidt (1994). "The kinetics and electrochemical rate-determining step of aqueous pyrite oxidation." Geochimica et Cosmochimica Acta 58(24): 5443-5454.

Winterbourn, M. J. (1998). "Insect Faunas of Acidic Coal Mine Drainages in Westland, New Zealand." New Zealand Entomologist 21(1): 65-72.

Winterbourn, M. J., W. F. McDiffett and S. J. Eppley (2000). "Aluminium and iron burdens of aquatic biota in New Zealand streams contaminated by acid mine drainage: effects of trophic level." Science of the Total Environment 254(1): 45-54.

Wolthers, M., L. Charlet and P. Van Cappellen (2008). "The surface chemistry of divalent metal carbonate minerals; a critical assessment of surface charge and potential data using the charge distribution multi-site ion complexation model." American Journal of Science 308(8): 905-941.

Xu, Y., L. Axe, T. Boonfueng, T. A. Tyson, P. Trivedi and K. Pandya (2007). "Ni(II) complexation to amorphous hydrous ferric oxide: An X-ray absorption spectroscopy study." Journal of Colloid and Interface Science 314(1): 10-17.

Yim, J. H., K. W. Kim and S. D. Kim (2006). "Effect of hardness on acute toxicity of metal mixtures using Daphnia magna: Prediction of acid mine drainage toxicity." Journal of Hazardous Materials 138(1): 16-21.

Younger, P. L., S. A. Banwart and R. S. Hedin (2002). Mine Water: Hydrology, Pollution, Remediation. Dordrecht, The Netherlands, Kluwer Academic Publishers. 


\section{Appendices}

Appendix 1. Neutralisation dose

A 1: AMD neutralisation dose by alkalinity reagent

\begin{tabular}{|c|c|c|c|}
\hline $\begin{array}{l}\text { Alkalinity dose } \\
\left(\mathrm{mg} / \mathrm{L} \mathrm{CaCO}_{3}\right)\end{array}$ & $\mathrm{NaOH}$ & $\begin{array}{c}\mathbf{p H} \\
\mathrm{Ca}(\mathrm{OH})_{2}\end{array}$ & $\mathrm{CaCO}_{3}$ \\
\hline 100 & 3.05 & & \\
\hline 200 & 3.37 & & 3.09 \\
\hline 300 & 3.87 & & \\
\hline 400 & 4.33 & & 3.6 \\
\hline 425 & 4.37 & & \\
\hline 450 & 4.39 & 4.44 & \\
\hline 475 & 4.43 & & \\
\hline 500 & 4.46 & 4.49 & \\
\hline 525 & 4.49 & & \\
\hline 550 & 4.53 & 4.55 & \\
\hline 575 & 4.57 & & \\
\hline 600 & 4.61 & 4.62 & 4.19 \\
\hline 625 & 4.66 & & \\
\hline 650 & 4.73 & 4.61 & \\
\hline 675 & 4.82 & & \\
\hline 700 & 4.94 & 4.75 & \\
\hline 725 & 5.3 & & \\
\hline 750 & 5.88 & 4.98 & \\
\hline 775 & 6.21 & & \\
\hline 785 & 6.48 & & \\
\hline 795 & 6.71 & & \\
\hline 800 & & 5.5 & 4.27 \\
\hline 805 & 6.88 & & \\
\hline 810 & & 5.97 & \\
\hline 815 & 7.01 & & \\
\hline 820 & & 6.54 & \\
\hline 825 & 7.21 & & \\
\hline 840 & & 6.59 & \\
\hline 845 & & 7 & \\
\hline 850 & 7.71 & & \\
\hline 850 & & 7.33 & \\
\hline 875 & 8.29 & & \\
\hline 900 & 8.97 & 8.02 & \\
\hline 950 & & 8.51 & \\
\hline 1000 & & 9.33 & 4.52 \\
\hline 1200 & & & 5.08 \\
\hline 1400 & & & 5.55 \\
\hline 1600 & & & 5.81 \\
\hline 1800 & & & 6.01 \\
\hline 2000 & & & 6.11 \\
\hline 2200 & & & 6.28 \\
\hline
\end{tabular}


Appendices

\section{Appendix 2. BTP Water Quality}

\section{A 2: BTP Turbidity}

\begin{tabular}{ccccccc}
\hline & \multicolumn{5}{c}{$\begin{array}{c}\text { Turbidity } \\
\text { (NTU) }\end{array}$} \\
\hline Time (min) & 30 & 60 & 120 & 30 & 60 & 120 \\
Control & - & - & - & - & - & - \\
SEHA20 before & - & - & - & - & - & - \\
SEHA20 after & $4.8(0.1)$ & $4.5(0.2)$ & $4.3(0.1)$ & - & $1.2(0.6)$ & $1.6(0.5)$ \\
SEHA20 during & - & - & - & - & - & - \\
SEHA100 before & - & - & - & - & - & - \\
SEHA100 after & $24.4(0.2)$ & $23.4(0.1)$ & $23.0(0.4)$ & - & - & $1.1(0.7)$ \\
SEHA100 during & - & - & - & - & - & - \\
AHA20 before & - & - & - & - & - & - \\
AHA20 after & $1.9(0.1)$ & $1.7(0.0)$ & $1.7(0.1)$ & - & - & - \\
AHA20 during & - & - & - & - & - & - \\
\hline
\end{tabular}

A 3: BTP SS

\begin{tabular}{ccc}
\hline & \multicolumn{3}{c}{$\begin{array}{c}\text { BTP SS } \\
(\mathbf{m g} / \mathrm{L})\end{array}$} \\
Control & $\mathrm{NaOH}$ & - \\
SEHA20 before & - & - \\
SEHA20 after & - & $5.2(1.3)$ \\
SEHA20 during & - & $2.8(1.4)$ \\
SEHA100 before & - & $5.2(0.3)$ \\
SEHA100 after & $18.4(0.8)$ & $3.0(1.1)$ \\
SEHA100 during & - & $3.3(0.7)$ \\
AHA20 before & - & - \\
AHA20 after & - & - \\
AHA20 during & $2.3(0.1)$ & $2.0(0.6)$ \\
\hline
\end{tabular}




\section{Appendix 3.MSS Water Quality}

\section{A 4: MSS Turbidity}

\begin{tabular}{ccccccc}
\hline & \multicolumn{5}{c}{ MSS Turbidity } \\
& $\mathbf{2}$ & $\mathbf{6}$ & $\mathbf{2 4}$ & $\mathbf{7 2}$ & $\mathbf{1 2 0}$ & $\mathbf{1 6 8}$ \\
\hline CaCO $_{3}$ & $23.2(1.7)$ & $12.7(0.9)$ & $4.1(0.6)$ & $1.1(0.3)$ & - & $1.6(0.3)$ \\
SEHA20 before & $24.2(0.5)$ & $13.8(1.0)$ & $6.5(2.0)$ & $2.3(1.1)$ & $1.2(0.6)$ & $1.2(0.9)$ \\
SEHA20 after & $4.3(0.8)$ & $\mathrm{n}$ & $3.3(1.3)$ & $1.2(0.5)$ & - & - \\
SEHA20 during & $10.5(0.8)$ & $6.1(0.1)$ & $2.2(0.4)$ & - & - & $2.3(1.6)$ \\
SEHA100 before & $20.0(3.7)$ & $17.3(4.0)$ & 6.60 .0 & $1.3(0.6)$ & $1.1(0.3)$ & $1.3(0.4)$ \\
SEHA100 after & $2.7(0.5)$ & $1.6(0.1)$ & $1.4(0.2)$ & $1.0(0.0)$ & - & - \\
SEHA100 during & $10.3(0.5)$ & $6.0(0.3)$ & $2.2(0.3)$ & $1.4(0.0)$ & - & - \\
AHA20 before & $34.1(1.8)$ & $19.7(1.8)$ & $5.4(0.3)$ & $1.5(0.3)$ & - & $1.5(0.3)$ \\
AHA20 after & $17.3(6.9)$ & $12.7(2.8)$ & $4.0(1.1)$ & $1.3(0.4)$ & $1.4(0.2)$ & - \\
AHA20 during & $10.3(1.8)$ & $5.4(1.8)$ & $3.0(1.4)$ & $1.6(0.5)$ & - & - \\
\hline
\end{tabular}

\begin{tabular}{ccccccc}
\hline & \multicolumn{7}{c}{ MSS Suspended Solids } \\
& $\mathbf{2}$ & $\mathbf{6}$ & $\mathbf{2 4}$ & $\mathbf{7 2}$ & $\mathbf{1 2 0}$ & $\mathbf{1 6 8}$ \\
\hline CaCO $_{3}$ & $17.0(0.3)$ & $9.6(0.3)$ & $3.6(0.6)$ & $2.8(0.3)$ & $2.5(1.0)$ & $2.4(0.9)$ \\
SEHA20 before & $18.5(0.1)$ & $4.9(6.9)$ & $3.6(1.1)$ & $3.1(0.4)$ & $2.6(0.3)$ & $3.7(0.1)$ \\
SEHA20 after & $4.6(0.6)$ & $\mathrm{n}$ & $3.7(0.7)$ & - & $3.5(0.4)$ & $3.1(0.1)$ \\
SEHA20 during & $11.6(0.3)$ & $7.2(0.6)$ & $4.1(0.1)$ & - & - & - \\
SEHA100 before & $16.0(1.7)$ & $10.9(0.7)$ & $4.6(0.6)$ & $2.6(1.1)$ & $3.7(0.4)$ & $3.6(0.0)$ \\
SEHA100 after & $4.1(0.1)$ & $3.8(1.1)$ & $2.3(2.7)$ & - & - & - \\
SEHA100 during & $8.7(0.4)$ & $5.6(0.3)$ & $2.0(0.0)$ & - & - & $2.1(0.1)$ \\
AHA20 before & $18.6(0.6)$ & $10.3(1.0)$ & $5.9(1.8)$ & $2.3(0.7)$ & $4.6(0.3)$ & $\mathrm{n}$ \\
AHA20 after & $13.7(0.7)$ & $8.4(0.6)$ & - & $2.4(0.9)$ & $2.8(0.3)$ & $2.3(0.4)$ \\
AHA20 during & $11.4(3.7)$ & $5.9(0.1)$ & $3.6(1.1)$ & - & $3.1(1.0)$ & $2.2(0.5)$ \\
\hline
\end{tabular}


Appendices

\section{Appendix 4. Polymer trails}

A 6: SEHA as a polymer Turbidity

\begin{tabular}{cccccc}
\hline $\begin{array}{c}\text { Time } \\
\text { (hrs) }\end{array}$ & $\mathbf{0}$ & $\mathbf{5}$ & $\mathbf{1 0}$ & $\mathbf{2 0}$ & $\mathbf{5 0}$ \\
\hline $\mathbf{0 . 5}$ & $58.3(8.0)$ & $35.5(2.4)$ & $31.6(7.1)$ & $8.0(0.8)$ & $22.1(2.5)$ \\
$\mathbf{1}$ & $45.4(4.2)$ & $32.2(4.7)$ & $24.3(4.1)$ & $6.0(1.6)$ & $11.8(0.8)$ \\
$\mathbf{2}$ & $37.5(1.1)$ & $26.7(3.5)$ & $19.4(7.0)$ & $5.0(0.2)$ & $8.0(3.2)$ \\
$\mathbf{3}$ & $29.1(0.2)$ & $21.2(8.1)$ & $19.7(2.3)$ & $4.5(0.2)$ & $8.6(1.0)$ \\
$\mathbf{6}$ & $20.7(8.1)$ & $18.1(2.9)$ & $19.3(7.5)$ & $2.9(0.0)$ & $6.1(2.1)$ \\
$\mathbf{2 4}$ & $13.3(1.1)$ & $5.2(0.2)$ & 5.00 .0 & $3.4(0.1)$ & $8.7(3.7)$ \\
\hline
\end{tabular}

A 7: SEHA as a polymer SS

\begin{tabular}{|c|c|c|c|c|c|}
\hline \multirow{2}{*}{$\begin{array}{l}\text { Time } \\
\text { (hrs) }\end{array}$} & \multicolumn{5}{|c|}{$\begin{array}{c}\text { SS }(\mathrm{mg} / \mathrm{L}) \\
\text { SEHA dose }(\mathbf{m g} / \mathrm{L})\end{array}$} \\
\hline & $\mathbf{0}$ & 5 & 10 & 20 & 50 \\
\hline 0.5 & $36.1(0.4)$ & $21.3(0.7)$ & $15.2(0.0)$ & $9.0(0.6)$ & $6.7(0.4)$ \\
\hline 1 & $28.3(0.4)$ & $20.0(3.4)$ & $13.1(2.7)$ & $6.7(1.3)$ & $5.4(0.3)$ \\
\hline 2 & $21.1(0.1)$ & $15.9(1.3)$ & $11.1(2.7)$ & $4.3(1.0)$ & $4.6(1.1)$ \\
\hline 3 & $16.9(1.3)$ & $13.0(0.6)$ & $10.4(1.7)$ & $3.4(1.1)$ & $5.6(1.4)$ \\
\hline 6 & $9.8(1.1)$ & $10.0(0.3)$ & $5.9(1.3)$ & $4.5(1.0)$ & $4.4(1.7)$ \\
\hline 24 & $7.0(1.7)$ & $6.4(1.1)$ & $6.0(0.0)$ & $5.2(0.3)$ & $4.7(0.7)$ \\
\hline
\end{tabular}

A 8: B570 Turbidity

\begin{tabular}{ccccc}
\hline $\begin{array}{c}\text { Time } \\
\text { (hrs) }\end{array}$ & \multicolumn{4}{c}{$\begin{array}{c}\text { Turbidity (NTU) } \\
\text { B570 dose (mg/L) }\end{array}$} \\
\hline $\mathbf{0 . 5}$ & $58.3(8.0)$ & $33.9(9.7)$ & $6.4(1.8)$ & $2.8(0.7)$ \\
$\mathbf{1}$ & $45.4(4.2)$ & $26.3(7.4)$ & $6.4(1.3)$ & $2.6(0.5)$ \\
$\mathbf{2}$ & $37.5(1.1)$ & $21.4(4.3)$ & $5.4(1.6)$ & $2.8(0.4)$ \\
$\mathbf{3}$ & $29.1(0.2)$ & $17.5(0.6)$ & $5.2(1.4)$ & $4.0(2.2)$ \\
$\mathbf{6}$ & $20.7(8.1)$ & $\mathrm{n}$ & $\mathrm{n}$ & $\mathrm{n}$ \\
$\mathbf{2 4}$ & $13.3(1.1)$ & $7.1(1.1)$ & $5.4(1.6)$ & $4.3(0.3)$ \\
\hline
\end{tabular}


Appendices

A 9: B570 SS

\begin{tabular}{ccccc}
\hline \multirow{2}{*}{$\begin{array}{c}\text { Time } \\
\text { (hrs) }\end{array}$} & \multicolumn{5}{c}{$\mathbf{S S}(\mathbf{m g} / \mathbf{L})$} \\
& $\mathbf{0} 570$ dose $(\mathbf{m g} / \mathbf{L})$ & \\
\hline $\mathbf{0 . 5}$ & $36.1(0.4)$ & $28.6(8.8)$ & $6.2(0.6)$ & $4.3(0.1)$ \\
$\mathbf{1}$ & $28.3(0.4)$ & $21.3(4.9)$ & $7.3(1.0)$ & $4.3(1.0)$ \\
$\mathbf{2}$ & $21.1(0.1)$ & $17.5(4.1)$ & $6.7(1.3)$ & $2.4(0.0)$ \\
$\mathbf{3}$ & $16.9(1.3)$ & $14.5(1.0)$ & $5.4(1.4)$ & $3.6(1.4)$ \\
$\mathbf{6}$ & $9.8(1.1)$ & $\mathrm{n}$ & $\mathrm{n}$ & $\mathrm{n}$ \\
$\mathbf{2 4}$ & $7.0(1.7)$ & $5.7(0.1)$ & $6.5(0.4)$ & $4.4(0.0)$ \\
\hline
\end{tabular}

A 10: B610 Turbidity

\begin{tabular}{ccccc}
\hline $\begin{array}{c}\text { Time } \\
\text { (hrs) }\end{array}$ & $\mathbf{5}$ & \multicolumn{2}{c}{ Turbidity (NTU) } \\
B610 dose (mg/L) & $\mathbf{0 . 0 1}$ & $\mathbf{0 . 0 5}$ & $\mathbf{0 . 1 0}$ \\
\hline $\mathbf{0 . 5}$ & $58.3(8.0)$ & $50.0(1.1)$ & $10.0(4.3)$ & $5.4(0.8)$ \\
$\mathbf{1}$ & $45.4(4.2)$ & $40.2(0.5)$ & $8.3(3.4)$ & $4.9(0.9)$ \\
$\mathbf{2}$ & $37.5(1.1)$ & $29.5(0.5)$ & $8.8(2.7)$ & $4.2(2.0)$ \\
$\mathbf{3}$ & $29.1(0.2)$ & $22.5(0.4)$ & $6.8(2.8)$ & $4.7(1.0)$ \\
$\mathbf{6}$ & $20.7(8.1)$ & $15.3(0.1)$ & $8.1(0.6)$ & $\mathrm{n}$ \\
$\mathbf{2 4}$ & $13.3(1.1)$ & $6.9(0.4)$ & $6.7(1.0)$ & $7.2(1.9)$ \\
\hline
\end{tabular}

A 11: B610 SS

\begin{tabular}{|c|c|c|c|c|}
\hline \multirow{2}{*}{$\begin{array}{l}\text { Time } \\
\text { (hrs) }\end{array}$} & \multicolumn{4}{|c|}{$\begin{array}{c}\text { SS }(\mathrm{mg} / \mathrm{L}) \\
\text { B610 dose }(\mathrm{mg} / \mathrm{L})\end{array}$} \\
\hline & 0 & 0.01 & 0.05 & 0.10 \\
\hline 0.5 & $36.1(0.4)$ & $38.7(1.3)$ & $8.4(3.7)$ & $4.8(2.0)$ \\
\hline 1 & $28.3(0.4)$ & $30.6(0.8)$ & $7.8(3.1)$ & $4.8(0.3)$ \\
\hline 2 & $21.1(0.1)$ & $21.3(0.4)$ & $8.0(2.0)$ & $4.2(0.6)$ \\
\hline 3 & $16.9(1.3)$ & $17.8(0.6)$ & $6.4(1.1)$ & $5.2(1.4)$ \\
\hline 6 & $9.8(1.1)$ & $12.2(0.3)$ & $6.9(0.1)$ & $\mathrm{n}$ \\
\hline 24 & $7.0(1.7)$ & $8.4(0.8)$ & $6.3(1.6)$ & $5.0(1.4)$ \\
\hline
\end{tabular}


Appendices

\section{Appendix 5. Control Standards}

A 12: Control standard: $\mathrm{NaOH}$

\begin{tabular}{cccccc}
\hline $\mathbf{p H}$ & $\mathbf{N i}$ & $\mathbf{Z n}$ & $\mathbf{C u}$ & $\mathbf{C d}$ & $\mathbf{P b}$ \\
\hline $\mathbf{6 . 0 9}$ & $0.47(0.05)$ & & $\mathrm{n}$ & $0.0034(0.0008)$ & $\mathrm{n}$ \\
$\mathbf{6 . 3 1}$ & $0.36(0.05)$ & $0.37(0.11)$ & $\mathrm{n}$ & $0.0017(0.0008)$ & $\mathrm{n}$ \\
$\mathbf{6 . 3 5}$ & $0.34(0.05)$ & $0.39(0.11)$ & $\mathrm{n}$ & $0.0027(0.0008)$ & $\mathrm{n}$ \\
$\mathbf{6 . 3 9}$ & $0.44(0.05)$ & $0.48(0.11)$ & $\mathrm{n}$ & $0.0022(0.0008)$ & $\mathrm{n}$ \\
$\mathbf{6 . 4 4}$ & $0.38(0.05)$ & $0.41(0.11)$ & $\mathrm{n}$ & $0.0018(0.0008)$ & $\mathrm{n}$ \\
$\mathbf{6 . 5 3}$ & $0.26(0.05)$ & $0.23(0.11)$ & $\mathrm{n}$ & $0.0017(0.0008)$ & $\mathrm{n}$ \\
$\mathbf{6 . 5 5}$ & $0.30(0.05)$ & $0.31(0.11)$ & $\mathrm{n}$ & $0.0025(0.0008)$ & $\mathrm{n}$ \\
$\mathbf{6 . 6 6}$ & $0.25(0.05)$ & $0.20(0.11)$ & $\mathrm{n}$ & $0.0013(0.0008)$ & $\mathrm{n}$ \\
$\mathbf{6 . 9 2}$ & $0.18(0.05)$ & $0.10(0.11)$ & $\mathrm{n}$ & $0.0010(0.0008)$ & $\mathrm{n}$ \\
$\mathbf{7 . 1 5}$ & $0.09(0.05)$ & $0.06(0.11)$ & $\mathrm{n}$ & $0.0010(0.0008)$ & $\mathrm{n}$ \\
$\mathbf{7 . 2 4}$ & $0.09(0.05)$ & $0.06(0.11)$ & $\mathrm{n}$ & - & $\mathrm{n}$ \\
\hline
\end{tabular}

A 13: Control standard: $\mathrm{Ca}(\mathrm{OH})_{2}$

\begin{tabular}{cccccc}
\hline $\mathbf{p H}$ & $\mathbf{5}$ & $\mathbf{Z}$ & $\begin{array}{c}\text { Metal Concentration } \\
(\mathbf{m g} / \mathbf{L})\end{array}$ & $\mathbf{C d}$ & $\mathbf{P b}$ \\
\hline $\mathbf{5 . 9 3}$ & $0.43(0.09)$ & $2.21(0.26)$ & $0.34(0.03)$ & $0.0078(0.0016)$ & $0.028(0.006)$ \\
$\mathbf{6 . 0 4}$ & $0.40(0.09)$ & $1.88(0.26)$ & $0.34(0.03)$ & $0.0059(0.0016)$ & $0.027(0.006)$ \\
$\mathbf{6 . 2 7}$ & $0.49(0.09)$ & $1.22(0.26)$ & $0.35(0.03)$ & $0.0067(0.0016)$ & $0.035(0.006)$ \\
$\mathbf{6 . 2 8}$ & $0.39(0.09)$ & $1.40(0.26)$ & $0.34(0.03)$ & $0.0078(0.0016)$ & $0.031(0.006)$ \\
$\mathbf{6 . 4 2}$ & $0.35(0.09)$ & $1.05(0.26)$ & $0.36(0.03)$ & $0.0044(0.0016)$ & - \\
$\mathbf{6 . 4 6}$ & $0.42(0.09)$ & $1.03(0.26)$ & $0.33(0.03)$ & $0.0043(0.0016)$ & $0.029(0.006)$ \\
$\mathbf{6 . 5 2}$ & $0.41(0.09)$ & $0.94(0.26)$ & $0.31(0.03)$ & $0.0048(0.0016)$ & $0.031(0.006)$ \\
$\mathbf{6 . 5 9}$ & $0.32(0.09)$ & $1.00(0.26)$ & $0.34(0.03)$ & $0.0052(0.0016)$ & - \\
$\mathbf{6 . 7 5}$ & $0.26(0.09)$ & $0.84(0.26)$ & $0.34(0.03)$ & $0.0040(0.0016)$ & $0.027(0.006)$ \\
$\mathbf{6 . 9}$ & $0.20(0.09)$ & $0.79(0.26)$ & $0.34(0.03)$ & $0.0049(0.0016)$ & - \\
$\mathbf{7 . 1 5}$ & $0.11(0.09)$ & & $0.32(0.03)$ & $0.0047(0.0016)$ & - \\
$\mathbf{7 . 3 5}$ & $0.23(0.09)$ & & $0.37(0.03)$ & $0.0044(0.0016)$ & $0.029(0.006)$ \\
\hline
\end{tabular}


Appendices

A 14: Control standard: $\mathrm{CaCO}_{3}$

\begin{tabular}{cccccc}
\hline $\mathbf{p H}$ & $\mathbf{N i}$ & $\mathbf{Z n}$ & $\mathbf{C u}$ & $\mathbf{C d}$ & $\mathbf{P b}$ \\
\hline $\mathbf{6 . 4 0}$ & $0.70(0.12)$ & $2.30(0.44)$ & $0.20(0.01)$ & $0.0039(0.0016)$ & $0.034(0.006)$ \\
$\mathbf{6 . 5 2}$ & $0.87(0.12)$ & $2.33(0.44)$ & $0.21(0.01)$ & $0.0042(0.0016)$ & $0.033(0.006)$ \\
$\mathbf{6 . 9 4}$ & $0.82(0.12)$ & $1.86(0.44)$ & $0.19(0.01)$ & $0.0037(0.0016)$ & $0.033(0.006)$ \\
$\mathbf{7 . 6 0}$ & $0.54(0.12)$ & $1.30(0.44)$ & $0.19(0.01)$ & $0.0029(0.0016)$ & $0.032(0.006)$ \\
$\mathbf{7 . 8 7}$ & $0.52(0.12)$ & $1.63(0.44)$ & $0.19(0.01)$ & $0.0032(0.0016)$ & $0.033(0.006)$ \\
$\mathbf{7 . 9 9}$ & $0.49(0.12)$ & $0.77(0.44)$ & $0.18(0.01)$ & $0.0026(0.0016)$ & $0.031(0.006)$ \\
\hline
\end{tabular}




\section{Appendix 6. BTP metal removal}

A 15: NaOH neutralised HS dosed metal concentrations

\begin{tabular}{|c|c|c|c|c|c|c|c|c|c|c|}
\hline & \multicolumn{10}{|c|}{ Metal Concentration (mg/L) } \\
\hline & Raw AMD & $\begin{array}{c}\text { SEHA20 } \\
\text { before }\end{array}$ & $\begin{array}{c}\text { SEHA20 } \\
\text { after }\end{array}$ & $\begin{array}{c}\text { SEHA20 } \\
\text { during }\end{array}$ & $\begin{array}{c}\text { SEHA100 } \\
\text { before }\end{array}$ & $\begin{array}{c}\text { SEHA100 } \\
\text { after }\end{array}$ & $\begin{array}{l}\text { SEHA100 } \\
\text { during }\end{array}$ & $\begin{array}{c}\text { AHA20 } \\
\text { before }\end{array}$ & $\begin{array}{c}\text { AHA20 } \\
\text { after }\end{array}$ & $\begin{array}{c}\text { AHA20 } \\
\text { during }\end{array}$ \\
\hline pH & 2.8 & 6.565 & 6.69 & 6.435 & 6.65 & 6.855 & 6.64 & 7.01 & 7.17 & 7.32 \\
\hline $\mathbf{C u}$ & $0.91(0.55)$ & $0.51(0.22)$ & $0.37(0.16)$ & $0.15(0.03)$ & $0.54(0.21)$ & $0.37(0.16)$ & $0.14(0.01)$ & $0.31(0.17)$ & $0.41(0.22)$ & $0.53(0.19)$ \\
\hline $\mathbf{Z n}$ & $4.04(0.59)$ & $0.82(0.22)$ & $0.53(0.12)$ & $0.58(0.19)$ & $0.59(0.16)$ & $0.42(0.08)$ & $0.24(0.03)$ & $0.27(0.00)$ & $0.62(0.37)$ & $0.67(0.03)$ \\
\hline $\mathbf{P b}$ & $0.086(0.062)$ & $0.048(0.019)$ & $0.039(0.016)$ & $0.029(0.005)$ & $0.050(0.017)$ & $0.039(0.016)$ & $0.030(0.003)$ & $0.051(0.018)$ & $0.056(0.026)$ & $0.063(0.017)$ \\
\hline
\end{tabular}

A 16: $\mathrm{Ca}(\mathrm{OH})_{2}$ neutralised HS dosed metal concentrations

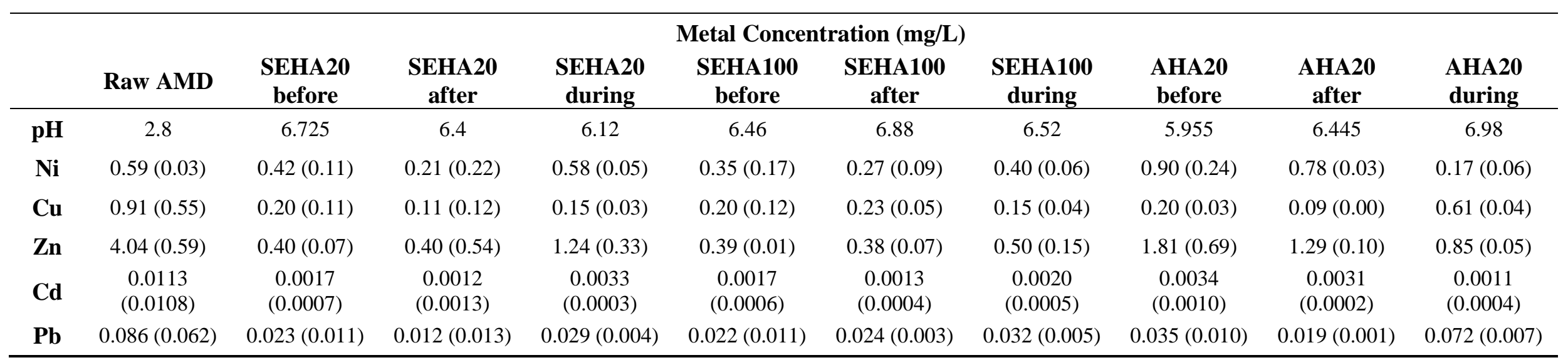


Appendices 